\title{
STRUCTURE DETERMINATION OF PEPTIDES WITH ANTIMICROBIAL ACTION
}

\author{
Dissertation \\ zur Erlangung des Doktorgrades \\ der Mathematisch-Naturwissenschaftlichen Fakultäten \\ der Georg-August-Universität zu Göttingen
}

vorgelegt von

Gábor Bunkóczi

aus Debrecen, Ungarn

Göttingen, 2004 
D 7

Referent: $\quad$ Prof. G. M. Sheldrick, PhD

Korreferent: Prof. Dr. R. Ficner

Tag der mündlichen Prüfung: 29.04.2004 


\section{ACKNOWLEDGEMENT}

I am indebted to Prof. George M. Sheldrick for providing an excellent scientific atmosphere and superior working conditions both on the instrumental and the theoretic level; I was often dependent on his wide knowledge, which he never hesitated to share with me when he saw the necessity. I would explicitly like to thank him for being such a good teacher; his seminars treated nearly all aspects of crystallography and were essential in developing my understanding of the method. I hope he can assist students in the future with as much commitment as he helped my work and wish him good health and less involvement in administration so he could better concentrate on scientific problems that concern him.

I am also obliged to Dr. László Vértesy and Matthias Schiell from Aventis Pharma Deutschland for believing in the importance of X-ray crystallographic investigations, for good advice and for supplying samples. I hope the cooperation between the two groups will continue smoothly after the retirement of Dr. Vértesy and produce as many astonishing results as before.

During my work, I was continuously supported not only in the lab but also at home by my wife, Judit É. Debreczeni, whose profound knowledge, skill, experience and kindness I highly esteem. I would explicitly like to thank her for reading the whole manuscript and drawing figures.

I am grateful to Madhumati Sevvana, Christine Schlicker, Drs. Regine Herbst-Irmer, Tim Grüne, Stephan Rühl and Trixie Wagner for finding and correcting numerous mistakes in the forthcoming thesis. I thank for the whole group, especially for Drs. Thomas R. Schneider and Isabel Usón for an informal working atmosphere and for discussions. I am grateful to Helmut Dehnhardt for his excellent technical assistance and for his free and easy manner. I would also like to express my thanks to everyone who supported me in the acquisition of the German language, especially to Stephan Müller.

At last, but not at least, I thank my parents for giving me free choice in selecting my future profession, for their constant support and tolerance. 


\section{LIST OF ABBREVIATIONS}

\begin{tabular}{|c|c|c|}
\hline AA & - & amino acid \\
\hline Ac & - & acetyl \\
\hline Aib & - & $\alpha$-aminoisobutyric acid \\
\hline AIDS & - & acquired immunodeficiency syndrome \\
\hline Ala & - & alanine \\
\hline Asn & - & asparagine \\
\hline Asp & - & aspartate \\
\hline Asx & - & asparagine or aspartate \\
\hline BESSY & - & Berliner Elektronenspeicherring \\
\hline $\mathrm{CC}$ & - & correlation coefficient \\
\hline CSD & - & Cambridge Structural Database \\
\hline $\mathrm{Dab}$ & - & 2,3-diaminobutyric acid \\
\hline Dec & - & decenoic acid \\
\hline DNA & - & deoxyribonucleic acid \\
\hline Dpg & - & 3,5-dihydroxyphenylglycine \\
\hline $\mathrm{EF}$ & - & elongation factor \\
\hline Et & - & ethyl \\
\hline fMet & - & formyl methinone \\
\hline GlcNAc & - & $\mathrm{N}$-acetylglucosamine \\
\hline Gln & - & glutamine \\
\hline Glu & - & glutamate \\
\hline Gly & - & glycine \\
\hline HAc & - & acetic acid \\
\hline HIV & - & human immunodeficiency virus \\
\hline Hyp & - & 4-hydroxyproline \\
\hline IF & - & initiation factor \\
\hline Itd & - & $\Delta 3$-isotetradecenoic acid \\
\hline Iva & - & isovaline \\
\hline Kyn & - & kynurenine \\
\hline Leu & - & leucine \\
\hline Lys & - & lysine \\
\hline
\end{tabular}




$\begin{array}{lll}\text { Map } & - & \beta \text {-methyl-aspartate } \\ \text { Me } & - & \text { methyl } \\ \text { Me-Glu } & - & \text { 3-methylglutamate } \\ \text { Mpg } & - & 4 \text {-monohydroxyphenylglycine } \\ \text { mRNA } & - & \text { messenger RNA } \\ \text { MurNAc } & - & \text { N-acetylmuramic acid } \\ \text { NaAc } & - & \text { sodium acetate } \\ \text { NADPH } & - & \text { nicotinamide adenine dinucleotide phosphate } \\ \text { NCS } & - & \text { non-crystallographic symmetry } \\ \text { Orn } & - & \text { ornithine } \\ \text { PBP } & - & \text { penicillin-binding protein } \\ \text { PDB } & - & \text { Protein Databank } \\ \text { PEG } & - & \text { polyethylene glycol } \\ \text { Phe } & - & \text { phenylalanine } \\ \text { Pheol } & - & \text { phenylalaninol } \\ \text { Pip } & - & \text { pipecolinic acid } \\ \text { Pro } & - & \text { proline } \\ \text { r.m.s.d. } & - & \text { residual mean-square deviation } \\ \text { RNA } & - & \text { ribonucleic acid } \\ \text { rRNA } & - & \text { ribosomal RNA } \\ \text { Ser } & - & \text { serine } \\ \text { sp } & - & \text { species (sing.) } \\ \text { spp } & \text { tryptophan } \\ \text { tRNA } & \text { uridine diphosphate } \\ \text { Trp } & - & \text { tridine triphosphate } \\ \text { UDP } & - & -\end{array}$




\section{MOLECULAR GRAPHICS}

Three-dimensional drawings of molecules were generated by MolScript (Kraulis, 1991) and rendered with Raster3D (Merritt \& Bacon, 1997). Electron density figures were generated with BobScript (Esnouf, 1999) and rendered with Raster3D or generated with PyMOL (DeLano, 2003) and rendered with PyMOL's built-in renderer. Molecular surfaces were also created using PyMOL. Figure 5.16 was drawn using XtalView (McRee, 1999) and rendered with Raster3D. 


\section{TABLE OF CONTENTS}

I. INTRODUCTION .................................................................................................. 1

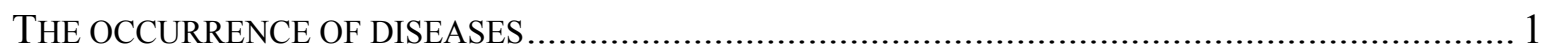

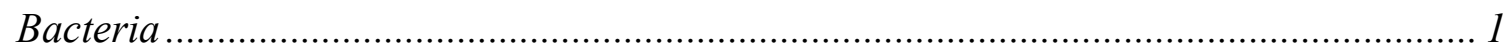

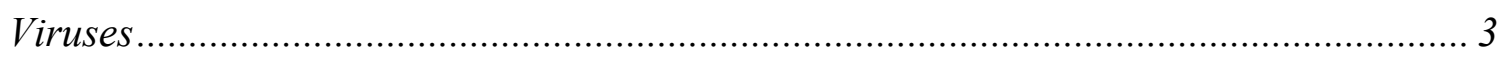

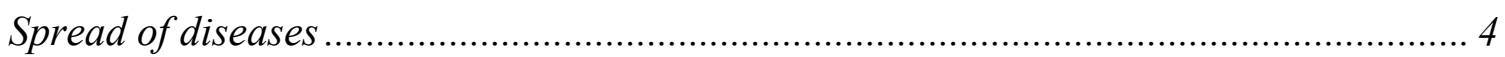

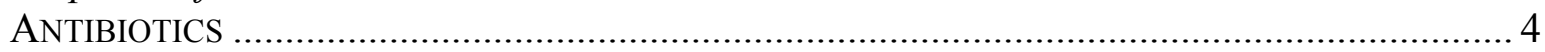

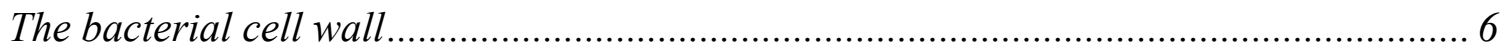

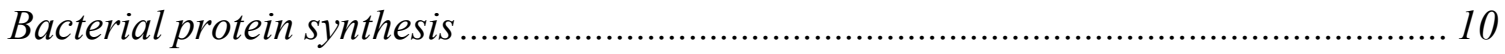

Other biosynthetic reactions targeted by antibiotics .................................................. 12

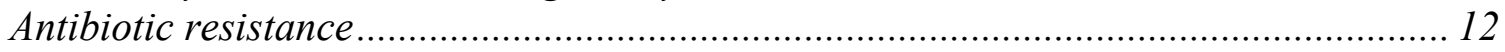

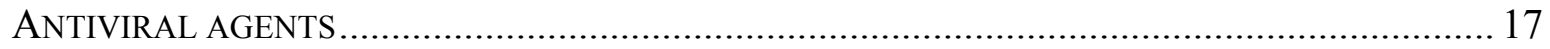

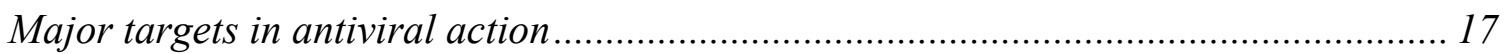

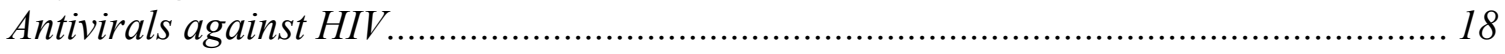

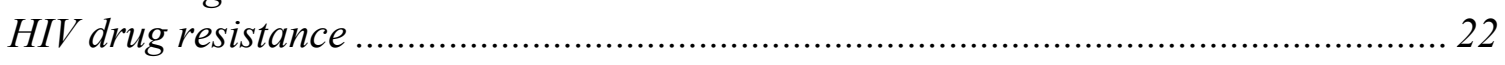

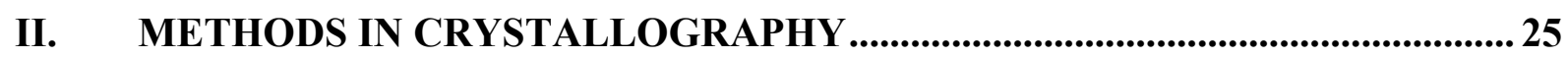

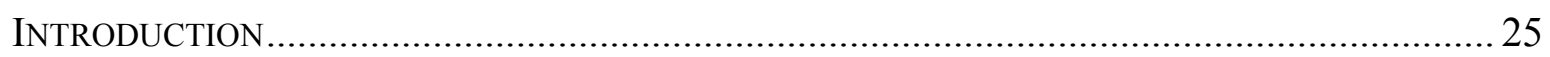

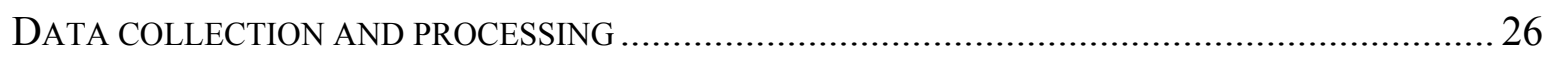

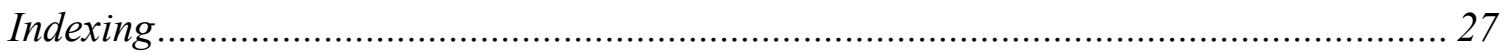

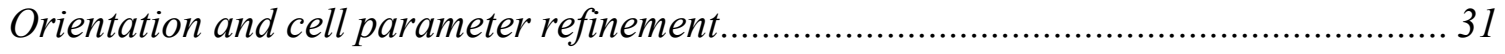

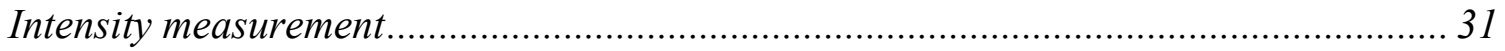

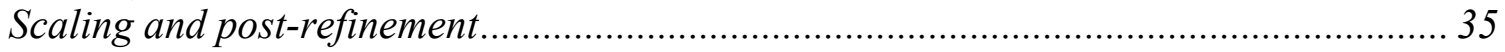

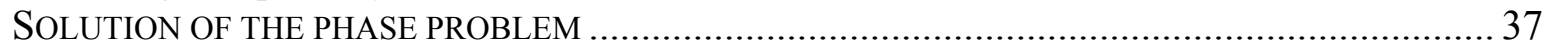

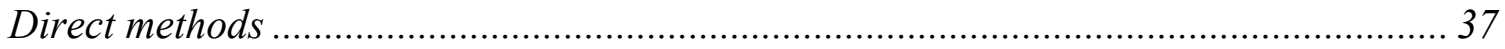

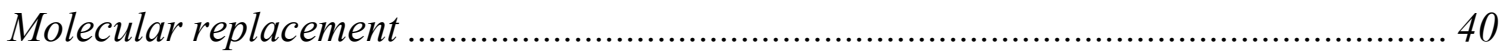

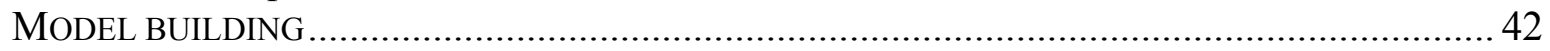

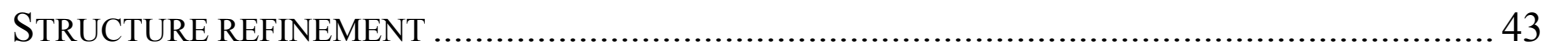

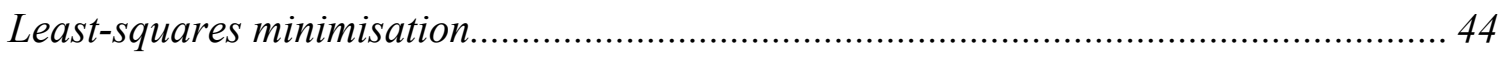

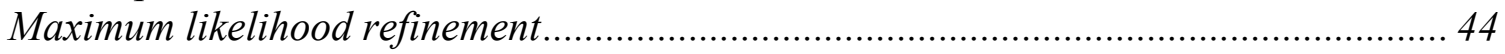

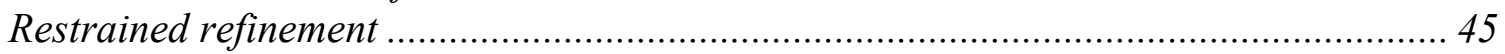

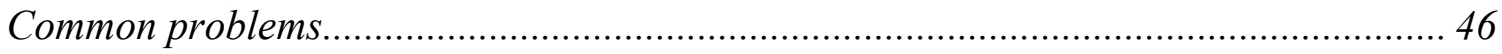

III. CRYSTAL STRUCTURES OF CEPHAIBOLS ..................................................... 47

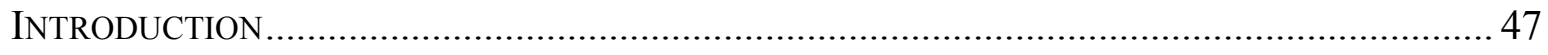

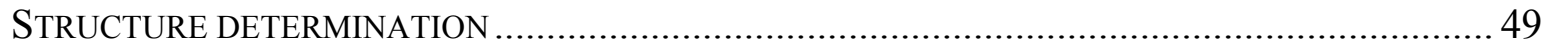

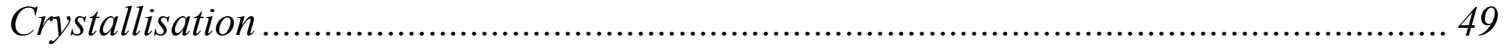

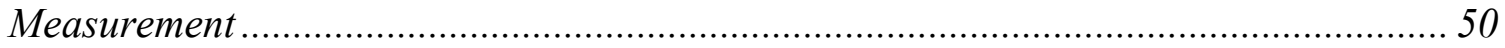

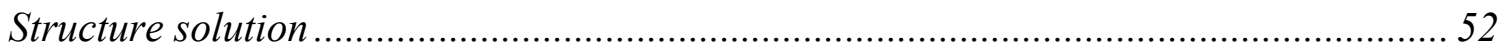

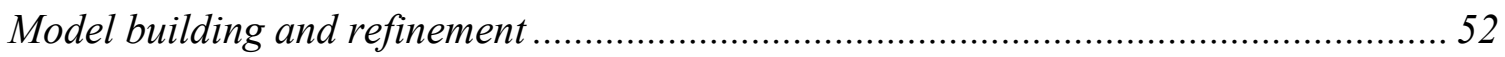

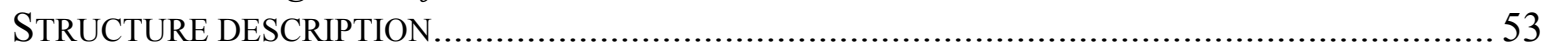

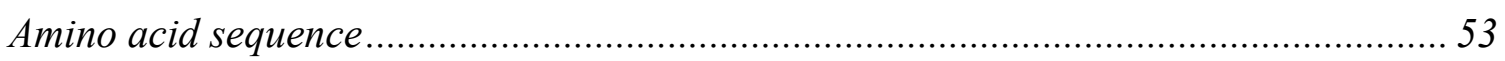

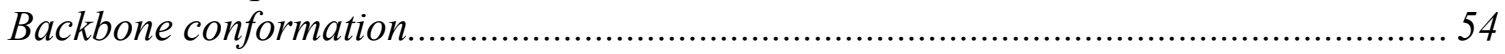

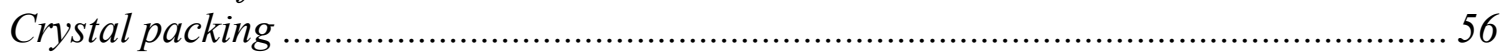

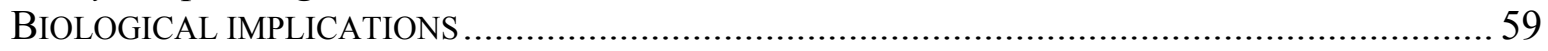

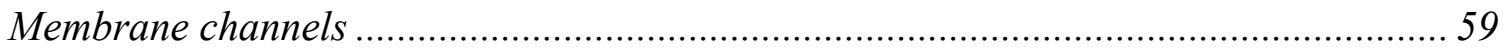

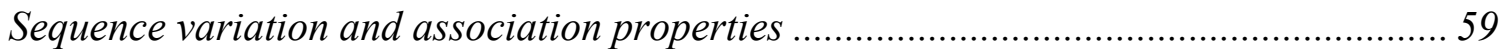

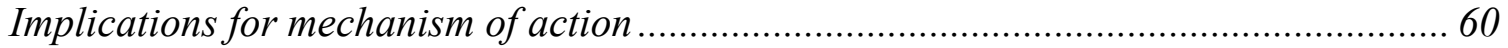




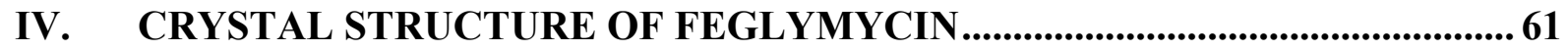

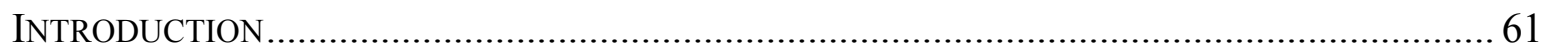

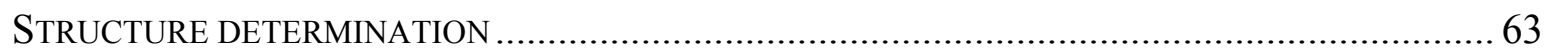

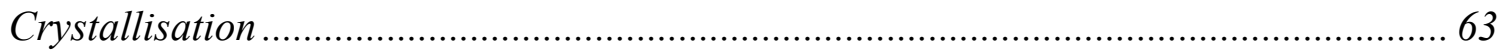

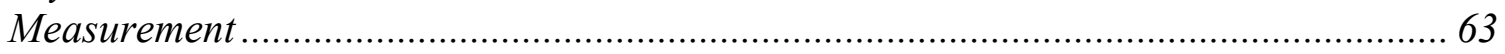

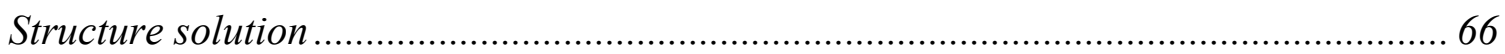

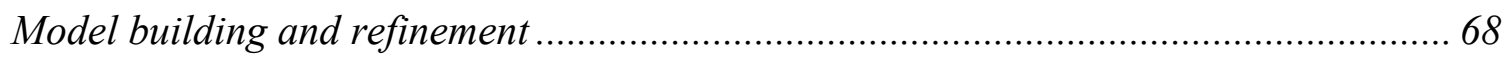

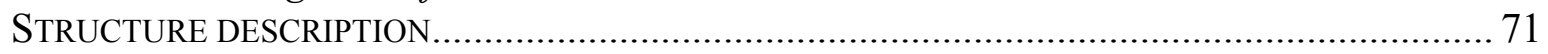

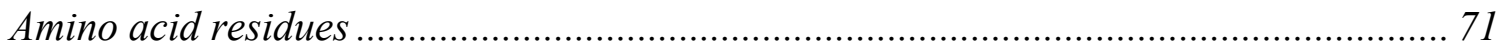

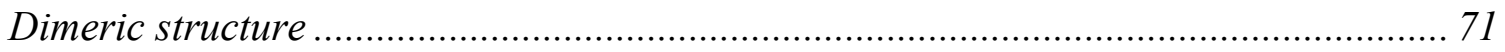

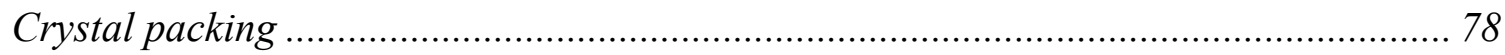

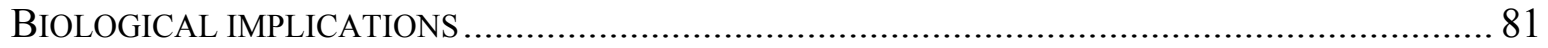

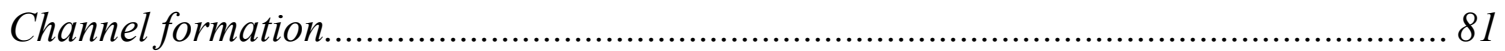

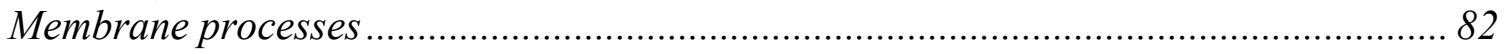

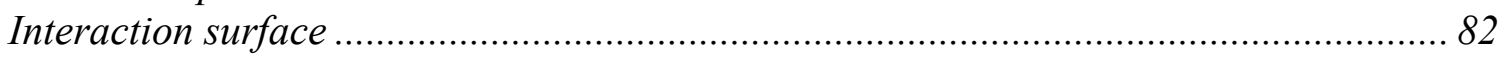

V. CRYSTAL STRUCTURE OF LIPOPEPTIDE ANTIBIOTICS..............................85

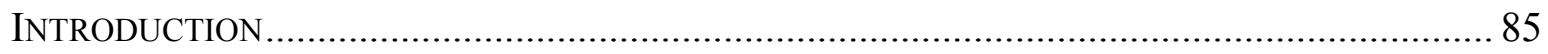

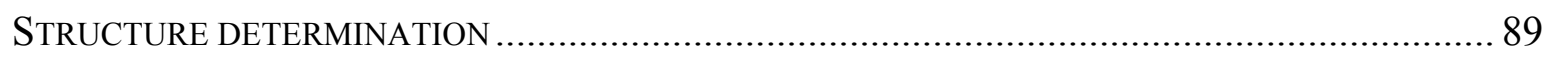

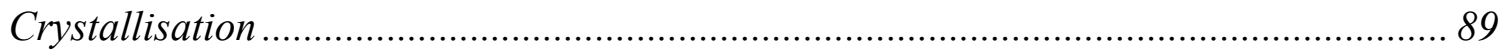

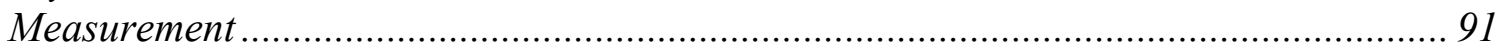

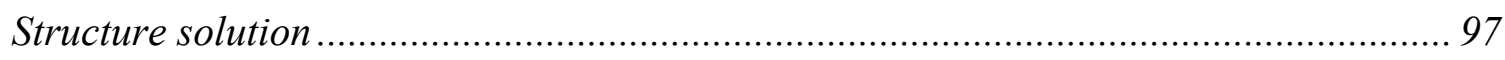

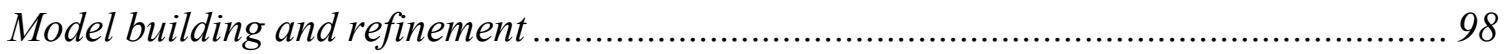

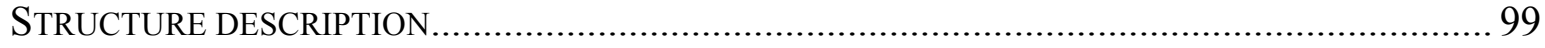

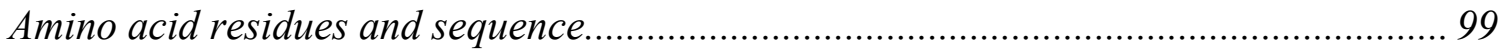

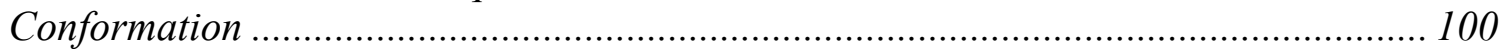

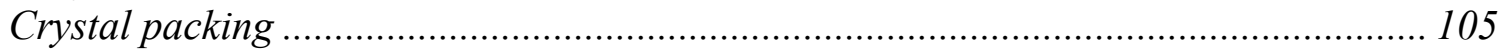

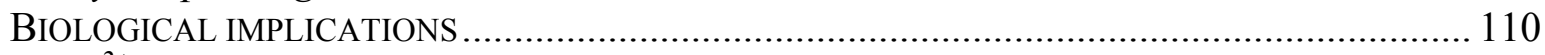

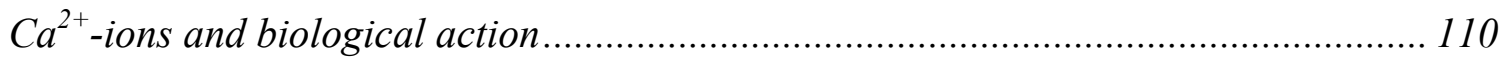

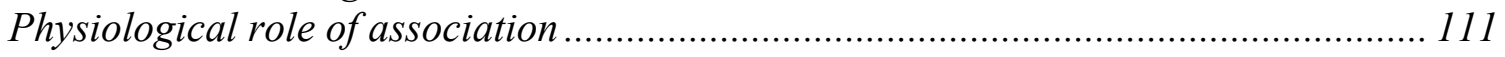

Implications for antibacterial action................................................................... 112

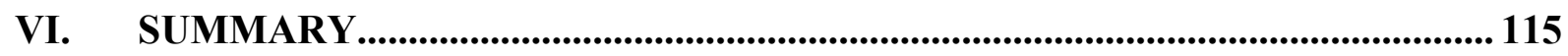

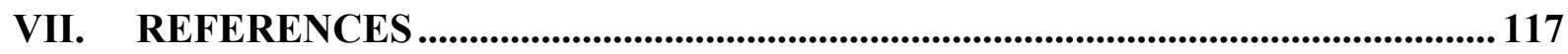




\section{INTRODUCTION}

\section{THE OCCURRENCE OF DISEASES}

The habitat of all living beings on Earth is the biosphere, which has a finite size and therefore interactions among the creatures are inherently present. One form of these interactions is symbiosis - the association of organisms with one another. Based on the type of association, symbiosis can further be divided into:

(a) mutualism, in which both members of the association benefit.

(b) commensalism, where there is no apparent benefit or harm to either member.

(c) parasitism, in which one member (the parasite) lives at the expense of the other (the host).

A disease is a manifestation of a host-parasite interaction, in which from the medical point of view the host is human and the parasite is a pathogen. In most of the cases, the pathogen is either a virus or a bacterium.

\section{Bacteria}

In humans, the presence of bacteria is a general phenomenon, as they are regularly found on all body surfaces; only the blood and the internal tissues are germ-free. Our normal intestinal flora contains approximately 450 aerobic and anaerobic bacterial species in such high concentrations $\left(10^{10}-10^{12}\right.$ bacteria per $\mathrm{ml}$ stool) that the number of symbiotic bacteria exceeds the number of human cells (Gracey, 1981; Lebek \& Cottier, 1992). Not all of this huge amount of bacteria can cause disease, though. A large percentage of bacteria found in the intestine is actually benevolent: they take up nutrients from the stool that humans cannot normally digest and convert them to more easily absorbable ones or to essential compounds that we cannot produce (Coates, 1975; Metges, 2000; Hooper et al., 2002). Another class of symbiotic bacteria includes species that are potential pathogens; these normally live in a commensal or parasitic relationship without producing a disease when the immune system, tissue resistance or anatomical barriers of the host are intact. Such microorganisms also include frequent causes of hospital infections like certain strains of Escherichia coli (Ochoa \& Cleary, 2003) or Enterococcus faecalis (Kayser, 2003). However, there are some bacteria that 
do not associate with their host except in the case of disease, like Bacillus anthracis or Vibrio cholerae.

\section{Bacterial infection}

If a bacterium, whether or not a constituent of the normal human flora, breaches the body surfaces and breaks into the sterile areas, an infection occurs. Although the infection normally implies colonisation, multiplication, invasion or persistence of a pathogen within a host, it is not equivalent to a disease, which refers to an infection that causes significant overt damage to the host.

There are several mechanisms in the body that provide protection against bacterial attacks. The first line of defence is undoubtedly the skin, which is a formidable barrier against bacterial penetration. In case a bacterium found its way through the skin, the immune system is responsible for destroying the intruder. Although these mechanisms function reliably in most of the cases, several bacteria have developed ways of overcoming or evading them (Dersch, 2003).

To cross the skin, bacteria can exploit a wound (e.g. vector bite, burn), and some microorganisms are capable of going through ubiquitous small skin abrasions or of direct penetration. However, most of the infections occur not on the skin, but through internal surfaces, like the respiratory, alimentary or urogenital tracts. Initially, only a small number of bacteria are deposited on these mucous surfaces. To survive, these have to compete with commensals that normally inhabit these surfaces, penetrate the mucus that covers them and adhere to epithelial cells (colonisation). Infectious organisms have developed specific means of attachment to host tissues, without which their colonies would normally be eliminated by normal host motility (Marra \& Isberg, 1996). The key players in this process are the bacterial adhesins, proteins that interact with specific host cell receptors (Klemm \& Schembri, 2000; Hauck, 2002). The inherent specificity in the adherence is responsible for apparent preference of particular bacteria to certain tissues or species and provides explanation for natural immunity of certain races within a species.

When a solid colony has already been established at the appropriate portal of entry, bacteria penetrate into the tissues by going through or between the epithelial cells or by destroying them (invasion). When in the tissue, bacteria have to withstand the attacks of the immune system and the specific immune response. Invasins, proteins taking part in the offensive, can effectively degrade the intercellular matrix to promote spread and act as nonspecific toxins to induce lysis of tissue cells in the immediate vicinity of bacterial growth. 
Finally, to be pathogenic, bacteria have to damage the tissues, which is normally achieved either by the production of a toxin or by lysing the host cell.

A bacterial infection does not necessarily imply the development of a disease. Some bacteria rarely cause disease if they infect, while certain microbes usually do. The ability of a bacterium to cause disease is generally referred to as its virulence or degree of pathogenicity. The manifesting virulence is the product of all the factors involved in the infection process (Smith, 1995). Research on bacterial pathogens aims to identify the genetic basis of these factors and to determine their function.

\section{Viruses}

Viruses are tiny particles that are built up of their genetic information (in the form of either RNA or DNA), a protein shell that protects the nucleic acids (nucleocapsid) and in enveloped viruses an additional lipid membrane. They extensively vary in their shape and complexity. Unlike bacteria, viruses are unable to function outside a host cell and scientists are divided whether or not a virus should be considered a living being.

All viruses have a common type of action. First, they recognize and then attach to the appropriate host cell by means of their protein shell and inject their genetic material into the cytosol. The injected genetic instructions recruit cell enzymes and begin to produce and assemble viral proteins. The assembled virus particles leave the host by either lysing and destroying it or by protruding from the cell and breaking away with a piece of lipid membrane.

Once inside the host cell, the virus does not necessarily start its own reproduction immediately. Some viruses, such as herpes and HIV, integrate their genetic material into the host cell's chromosomes, silently wait and allow the host cell to reproduce. When an environmental or predefined genetic signal arrives, the sleeping viral genes awake and take over the host's protein synthetic machinery.

Viruses exhibit remarkable host cell specificity. Upon approach towards the host cell, viruses require a specific receptor molecule that is complementary to the coat protein. This dependency on the presence of a certain protein places the main restriction on the type of cells a virus can infect (Baranowski et al., 2001). 


\section{Spread of diseases}

Although markedly different in structure and function, there are common mechanisms for transmitting a bacterial or a viral infection. In general, pathogens are transmitted either directly (e.g. through physical contact or body fluids) or with an appropriate carrier (contaminated food, water or insects). Preventing the transmission is probably the most important way of combatting a disease.

\section{ANTIBIOTICS}

Antibiotics were originally compounds isolated from soil microorganisms that were selectively toxic to other microbes. The group Streptomyces were found to be especially active in synthesising them and investigation of this family led to the discovery of several classes of antimicrobial compounds (e.g. streptomycin, tetracycline and erythromycin). The exact role of these compounds in the survival and economy of the producing organisms has not yet been clarified, though (Williams et al., 1989). As the public need for antibiotics increased and bacterial resistance become widespread, semisynthetic antibiotics (e.g. nonhydrolysable penicillins) were also introduced into medical practice and now there exist several classes of completely synthetic compounds with antimicrobial properties (e.g. fluoroquinolones). Some commercially available antibiotics are listed in Table 1.1.

All antibiotics possess a common principle in their mechanism of action: they achieve selective toxicity against bacteria by inhibiting an important step in their biosynthetic machinery that is not present or markedly different in eukaryotes. Such targets emerge by divergent evolutionary strategies followed by prokaryotes and eukaryotes, and may be divided into two classes. On one side, bacteria have introduced new structural substances that were unnatural and thus resistant against common eukaryotic degradation mechanisms. On the other side, certain key actors in the biosynthetic machinery of eukaryotes, although bearing a common ancestor with that of prokaryotes, have undergone substantial alteration in the course of evolution or have been replaced by more advanced variants. Major targets of antibiotics include bacterial cell-wall and protein synthesis (Figure 1.1). 


\begin{tabular}{lcc}
\hline Antibiotic & $\begin{array}{c}\text { Antimicrobial } \\
\text { spectrum }\end{array}$ & \multicolumn{1}{c}{ Mode of action } \\
\hline $\boldsymbol{\beta}$-lactams & Gram+ \\
Penicillin G & \\
Ampicillin & $\left.\begin{array}{l}\text { Gram+ } \\
\text { Gram- } \\
\text { Cefotoxime (cephalosporin) } \\
\text { Imipenem (carbapenem) } \\
\text { Aztreonam (monobactam) }\end{array}\right\}$ \\
\hline
\end{tabular}

\section{Glycopeptides}

Vancomycin

Teicoplanin

Gram+

Inhibit transpeptidation in cell-wall synthesis by binding the substrate

\section{Aminoglycoside

Gentamycin

Gram+ aerobic Gram-

Inhibit protein synthesis by blocking the $70 \mathrm{~S}$ initiation complex

\section{Tetracyclines}

$\left.\begin{array}{l}\text { Tetracycline } \\ \text { Doxicycline }\end{array}\right\} \begin{aligned} & \begin{array}{l}\mathrm{Gram}^{+} \\ \mathrm{Gram}^{-}\end{array}\end{aligned} \begin{aligned} & \text { Inhibit protein synthesis by blocking } \\ & \text { tRNA binding sites on the ribosome }\end{aligned}$

\section{Oxazolidinones}

\begin{tabular}{|c|c|c|}
\hline Linezolid & Gram + & $\begin{array}{l}\text { Inhibit initiation of protein synthesis } \\
\text { by binding the } 50 \text { S ribosomal subunit }\end{array}$ \\
\hline \multicolumn{3}{|l|}{ uinolones } \\
\hline Ciprofloxacin & $\begin{array}{l}\text { Gram+ } \\
\text { Gram- }\end{array}$ & $\begin{array}{l}\text { Inhibit transcription by preventing } \\
\text { the action of topoisomerases/gyrases }\end{array}$ \\
\hline
\end{tabular}

$\left.\begin{array}{lcl}\begin{array}{l}\text { MLS-antibiotics } \\ \text { Erythromycin } \\ \text { Lincomycin }\end{array} & \begin{array}{c}\text { Gram+ cocci } \\ \text { Gram- anaerobes } \\ \text { Chloramphenicol }\end{array} & \text { Gram+, Gram- }\end{array}\right\} \begin{aligned} & \text { Inhibit transpeptidase acitivity of the } \\ & \text { ribosome }\end{aligned}$

Table 1.1

Antimicrobial spectrum and action mechanism of selected members of commercially available antibiotic classes. 


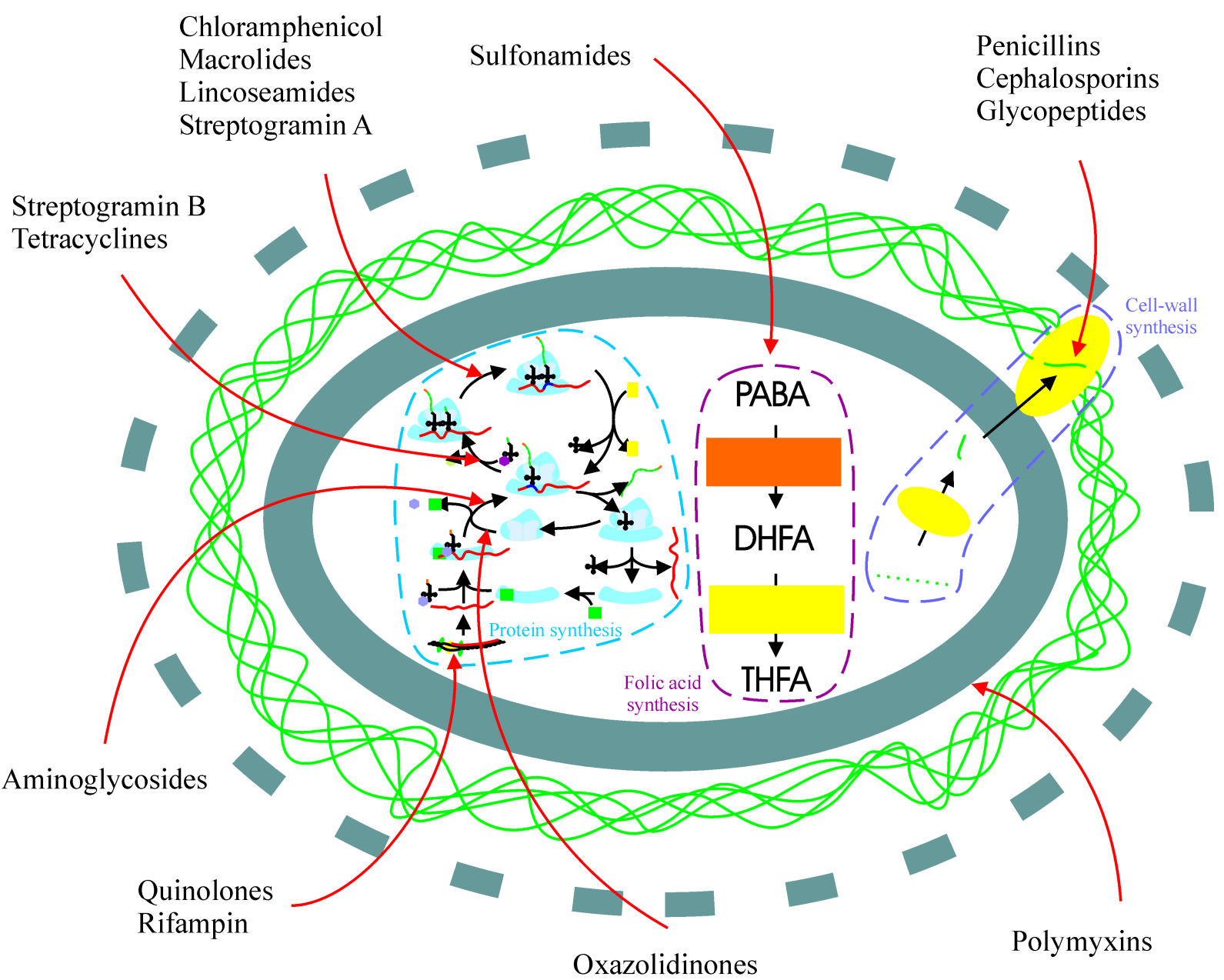

Figure 1.1

Biochemical reactions in bacteria targeted by antibiotic classes. Major targets are the protein, the cell-wall and the folic acid biosynthesis, but antibiotics having different action mechanism (e.g. permeabilisation of the cell membrane) are also known. PABA: para-aminobenzoic acid, DHFA: dihydrofolic acid, THFA: tetrahydrofolic acid. See Figures 1.2 and 1.3 for a more detailed reaction scheme of cell-wall biosynthesis and Figure 1.4 for that of protein synthesis.

\section{The bacterial cell wall}

The material of the bacterial cell wall, peptidoglycan or murein, is a polymer of unusual chemical structure that provides strength and rigidity to bacterial cells (Gale et al., 1981). The novel features of peptidoglycan, namely the presence of D-amino acids and $\gamma$-peptide bonds, are responsible for its chemical and biological resistance, but also for its vulnerability (Neuhaus \& Hammes, 1981). Disruption of the peptidoglycan layer results in cell lysis and subsequent death due to the high internal osmotic pressure present in bacterial cells. 
As peptidoglycan is an essential substance for bacteria in maintaining cell integrity, it is not surprising that most naturally occurring antibiotics inhibit enzymes taking part in its biosynthesis (Waxmann \& Strominger, 1983; Barna \& Williams, 1984; Frère \& Joris, 1985; Reynolds, 1989).

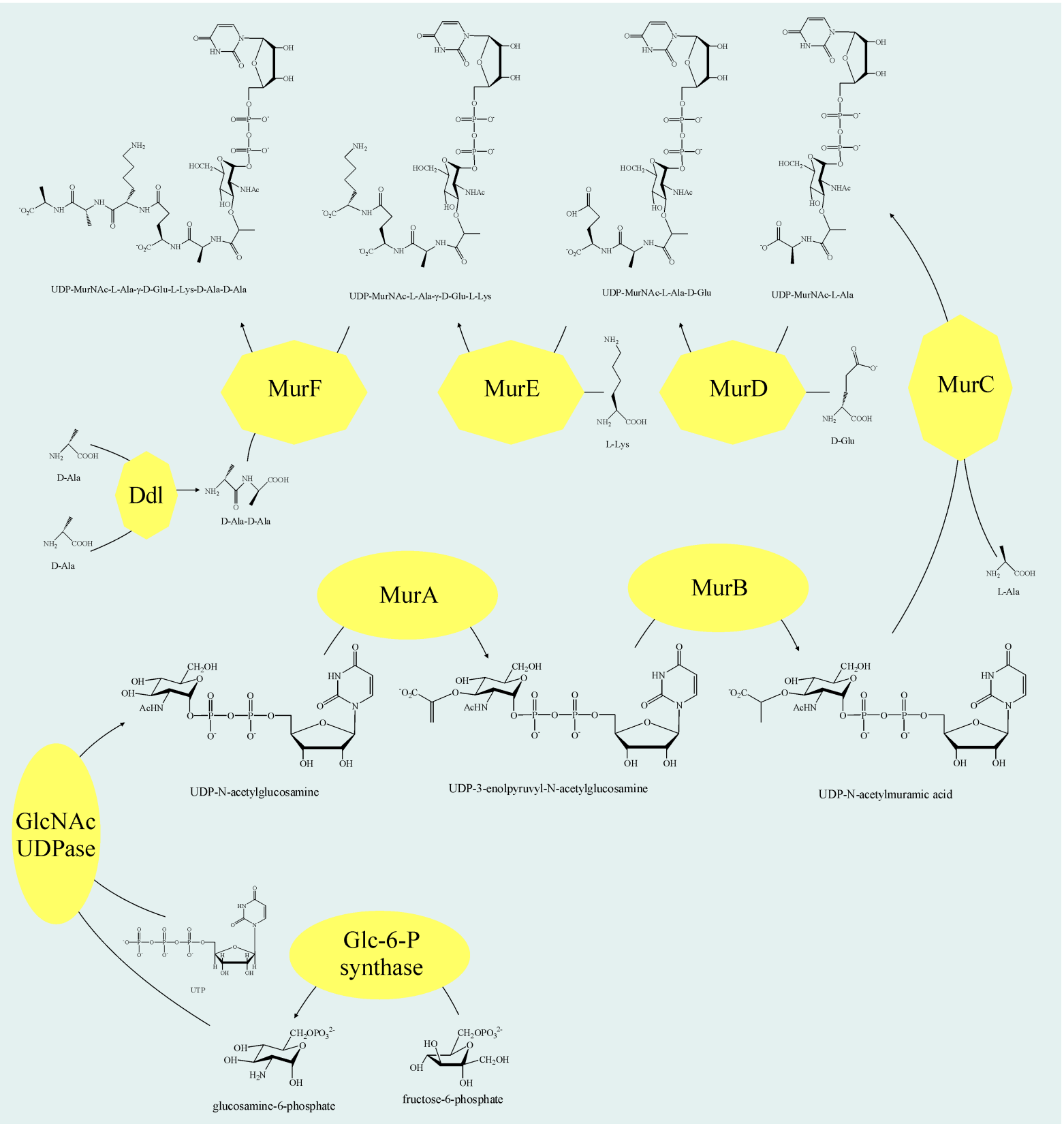

\section{Figure 1.2}

Biosynthesis of the bacterial cell wall I: reactions in the cytosol. Participating enzymes and compounds are designated according to conventional nomenclature or after the corresponding gene. The cell-wall pentapeptide corresponds to that of Gram-positive bacteria; in Gram-negative organisms L-Lys is replaced by meso-diaminopimelate. 
The functional murein has a layered structure built up by glycan strands, which are cross-linked through peptide chains. The glycan strands consist of alternating residues of $\mathrm{N}$-acetylmuramic acid (MurNAc) and N-acetylglucosamine (GlcNAc) linked by $\beta-1,4-$ glycosidic bonds. Peptide chains are connected to the D-lactyl group of MurNAc and join neighbouring glycan strands by means of a 9-residue sequence. Biosynthesis of murein proceeds in two stages. First, the GlcNAc-MurNAc-L-Ala- $\gamma-D-G l u-X-D-A l a-D-A l a$ monomer (where $\mathrm{X}$ is meso-diaminopimelate in Gram-negative and L-Lys in Gram-positive bacteria) is assembled in the cytosol, then transported across the cell membrane and added to the continuously growing peptidoglycan layer (Schleifer \& Kandler, 1972; Kamiryo \& Matsuhashi, 1972).

Biosynthesis of the monomer utilises the primary metabolite fructose-6-phosphate, which is converted into glucosamine-6-phosphate by the corresponding synthetase, acetylated at the amino group and linked to a UDP carrier (Figure 1.2). The resulting UDP-GlcNAc is further converted by UDP-GlcNAc enolpyruvyl transferase (encoded by the MurA gene) followed by the reduction of the enol to a lactyl group by the NADPH-dependent MurBreductase and yields UDP-MurNAc.

The sequence of the pentapeptide moiety is not DNA-encoded and the synthesis follows a non-ribosomal pathway directed by the specificity of amino acid ligases that catalyse subsequent steps. The reaction starts with the MurC enzyme that adds an L-Ala residue to the lactyl group of UDP-MurNAc, and proceeds through the addition of D-Glu (by the MurD enzyme) to the C-terminal and then meso-diaminopimelate/L-Lys (MurE enzyme) to the $\gamma$-carboxylate of D-Glu. The two D-Ala residues that complete the pentapeptide are first linked by a D-Ala-D-Ala ligase (ddlA or ddlB gene) and then connected to the N-terminus of UDP-MurNAc-tripeptide by the MurF enzyme. The required D-amino acids are either synthesised from the corresponding L-amino acid or from an $\alpha$-keto acid. The assembled precursor is then linked to an undecaprenyl lipid carrier by phospho-MurNAc-pentapeptide translocase (encoded on the MraY gene) and an additional GlcNAc is attached to the 4-OH group of MurNAc by MurG transferase (Figure 1.3). The complete lipid-II precursor is finally translocated across the cell membrane by an unknown mechanism and processed by penicillin-binding proteins (PBPs), which first add the precursor to the growing glycan strands (glycosyltransferase activity) and then cross-link the peptides to yield interstrand peptide brides (transpeptidase activity). 


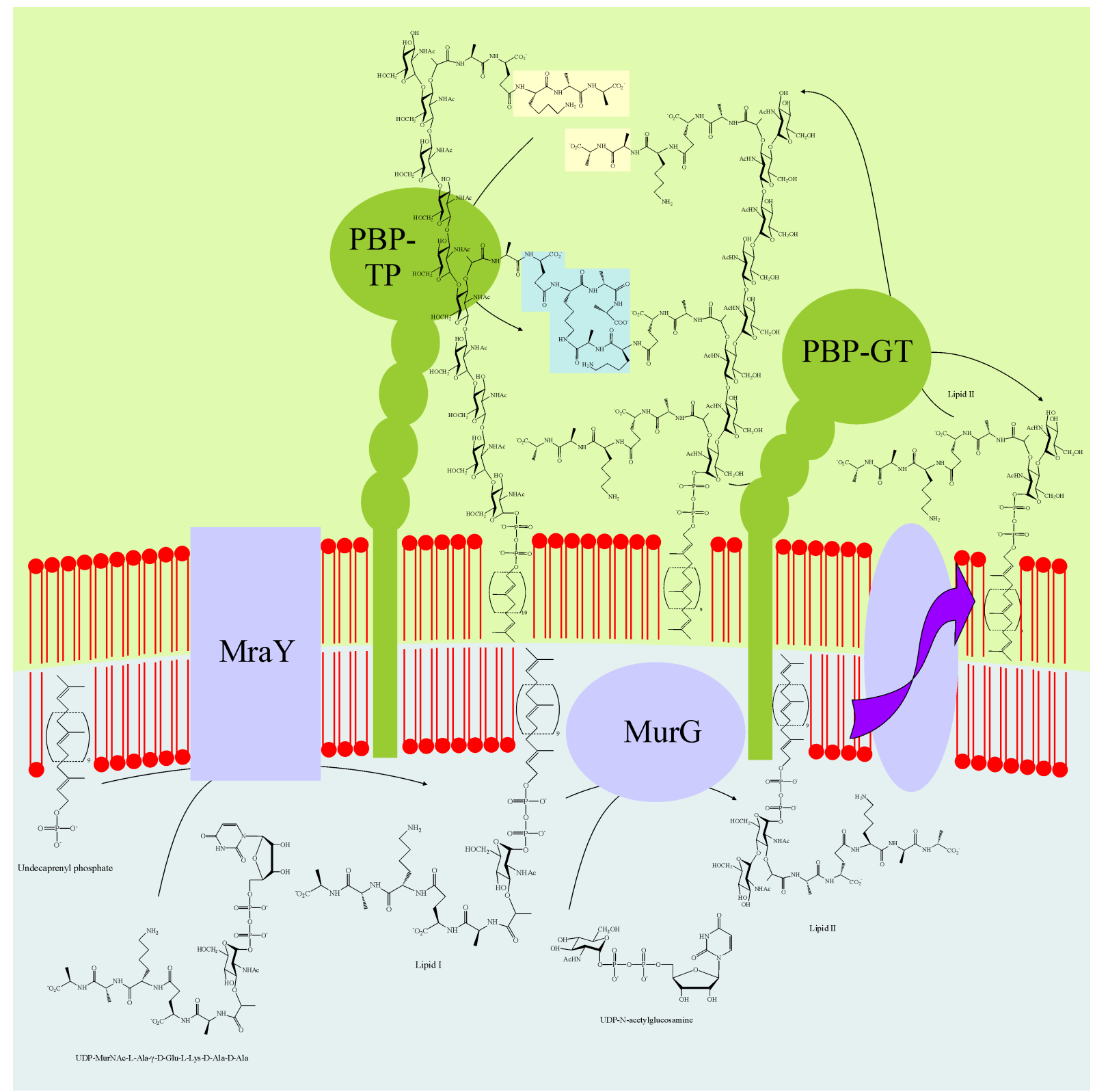

\section{Figure 1.3}

Biosynthesis of the bacterial cell wall II: reactions involving the cell membrane. PBP: penicillinbinding protein, GT: glycosyltransferase, TP: transpeptidase. The remaining enzymes are named after the encoding gene. The "flippase" enzyme has not been identified yet. The cell-wall building block corresponds to that of Gram-positive bacteria; in Gram-negative organisms L-Lys is replaced by meso-diaminopimelate.

Although murein biosynthesis consists of several steps, antibiotics used in current medical practice are limited in their action to transpeptidase inhibitors. The most important group is indisputably the one of $\beta$-lactams, as this group is present on the market not only with the most variants, but also with the highest quantity. Based on their chemical structure, 
$\beta$-lactams may be classified into penicillin and ampicillin derivatives, cephalosporines, carbapenems and monobactams, which all use the same principle for antimicrobial action (a four-member lactam ring that can react with and thus inactivate the transpeptidase domain of the PBP) and differ only in pharmacokinetic parameters and antimicrobial spectrum. The second main group comprises glycopeptide antibiotics, which inhibit transpeptidation by binding the D-Ala-D-Ala terminus of the pentapeptide moiety, therefore preventing approach and subsequent action of the transpeptidase enzyme (Chatterjee \& Perkins, 1966; Reynolds, 1989). Novel agents that target distinct steps of peptidoglycan synthesis are in development (Bugg \& Walsh, 1992).

\section{Bacterial protein synthesis}

The ribosomal protein synthesis machinery found in bacteria is notably different from the one in eukaryotes and many antibiotics function by inhibiting one or more essential reactions in it. Synthesis of a protein starts generally with transcription of genetic information encoded in the DNA into messenger RNA (Figure 1.4). This process implies the action of topoisomerases that untwist the DNA to make it accessible for the RNA polymerase enzyme, which creates a complementary RNA-copy of the DNA. The completed mRNA chain then binds an inactive $30 \mathrm{~S}$ ribosome subunit and positions the starting AUG codon to the partial P-site of the ribosome. A tRNA molecule with a formylated methionine carried by initiation factor IF-2 can now bind to the P-site. Upon binding, the IF-3 initiation factor preventing the $30 \mathrm{~S}$ subunit from premature association with the 50S dissociates, and the 50S subunit joins in. After this step the ribosome is operational and begins to synthesise the encoded protein by adding the amino acids encoded by the codons and carried by the complementary tRNA and then repositioning itself one codon further (translocation). The aminoacyl-tRNA is carried by elongation factor (EF) Tu and translocation is initiated by EF-G. When a stop codon is reached, the ribosome releases the polypeptide chain, detaches from the mRNA and dissociates into subunits (Noller, 1991). 


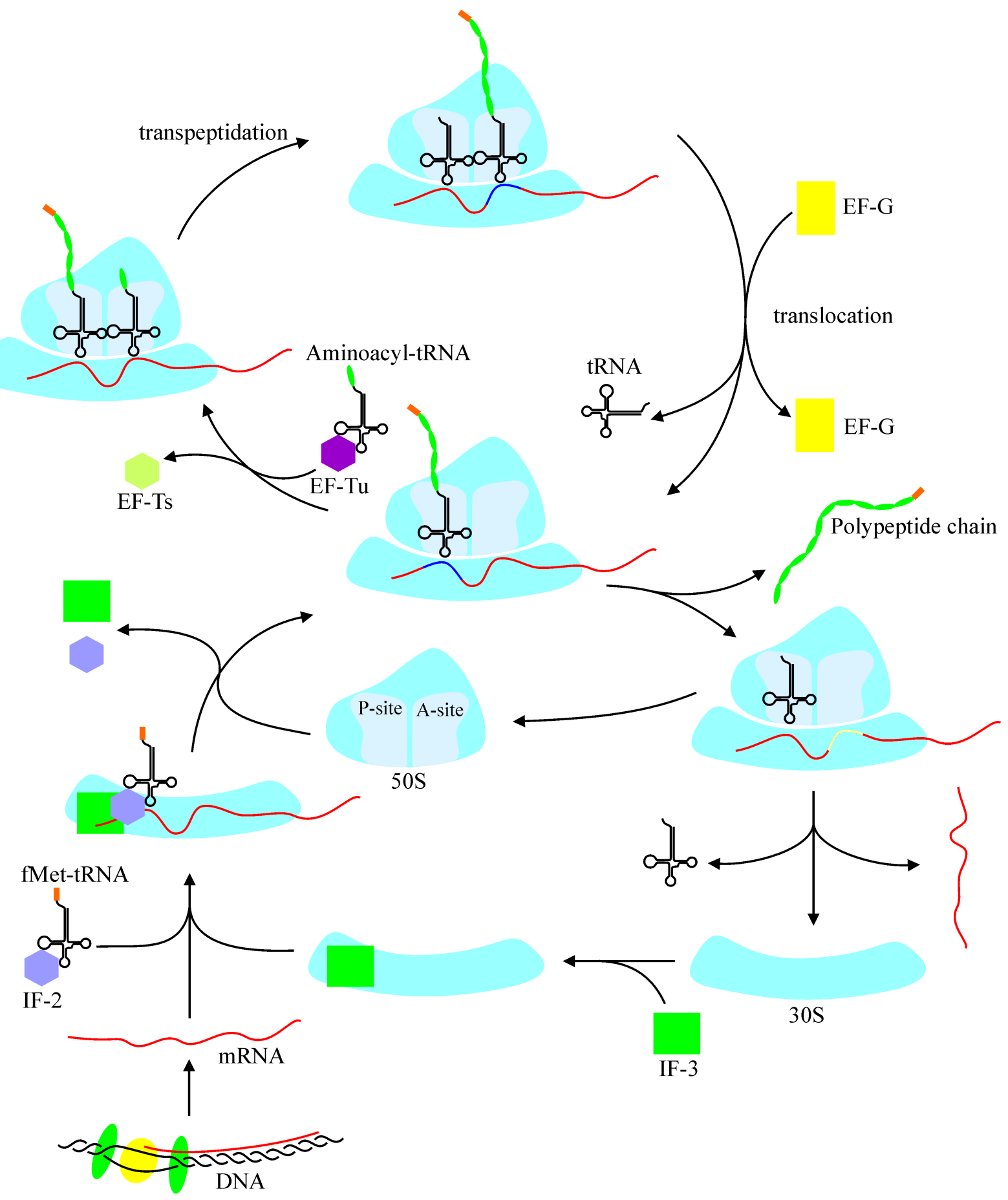

Figure 1.4

Steps of protein synthesis in bacteria. The process starts with transcription of the gene into an mRNA and ends with a polypeptide chain and the intact mRNA. This can be reused and employed for the synthesis of the same protein till it gets degraded. 
Antibiotics that interfere with bacterial protein synthesis include (Figure 1.1):

(a) quinolones prevent the function of topoisomerase IV and DNA-gyrase and thus inhibit transcription (Emmerson \& Jones, 2003).

(b) rifampin inhibits the RNA-polymerase (Furesz, 1970).

(c) oxazolidinones prevent initiation of the ribosome (Norrby, 2001; Rehm, 2002).

(d) aminoglycosides bind to the $16 \mathrm{~S}$ rRNA of the $30 \mathrm{~S}$ ribosomal subunit and block the initiation complex (Kotra et al., 2000).

(e) tetracyclines interact with aminoacyl-tRNA binding sites present on the 30S subunit and reduce their binding affinity (Durckheimer, 1975).

(f) MLS antibiotics (macrolides, lincosamides and streptogramines) bind the 23S rRNA of the 50S subunit and block peptidyltransferase activity (Di Giambattista et al., 1989; Cocito et al., 1997).

(g) chloramphenicol also inhibits the peptidyltransferase activity (Yunis, 1988).

However, as human cells also contain organelles that have bacterial origin (e.g. mitochondria) and where the bacterial protein synthetic machinery is partially active, these antibiotics may damage those as well. As murein synthesis inhibitors act on the cell surface and do not depend on transport across the membrane for antimicrobial action, they are not expected to cause side effects as frequently as protein biosynthesis inhibitors.

\section{Other biosynthetic reactions targeted by antibiotics}

Less common antibiotics in medical practice include the group of sulfonamides (Seydel, 1968), which inhibit the synthesis of tetrahydrofolic acid, an essential cofactor in nucleotide and amino acid biosynthesis, and polymyxins (Hermsen et al., 2003), which interact with the cell membrane and cause leakage of small molecules (Figure 1.1).

\section{Antibiotic resistance}

The increased use of antibiotics in medical practice constitutes an evolutionary force that drives bacteria to develop resistance mechanisms against these antimicrobial compounds (Levy, 1998). As most of the antibiotics have natural origin or mimic those isolated from natural sources, resistance mechanisms naturally exist in antibiotic-producing microorganisms such as Streptomyces or in soil bacteria, against which these substances were originally directed (Cundliffe, 1984). The genes for withstanding antibiotics are therefore inherently 
present in nature, but it is the appearance of resistance genes in clinical isolates of infectious bacteria that gives rise to considerable concern (Neu, 1992).

\section{Major resistance mechanisms}

Bacteria have developed four general means of withstanding the exposure to antibiotics (Hayes \& Wolf, 1990):

(a) inactivation or reduced metabolic activation of the antibiotic. This mechanism is responsible for low-level resistance against $\beta$-lactams and high-level resistances against aminoglycosides, lincosamides, streptogramins and chloramphenicol (Table 1.2).

(b) prevention of access to the target or active removal of the antibiotic from the cytosol (drug sequestration or efflux). Examples for this mechanism include the natural resistance of certain Gram-positive bacteria to $\beta$-lactam antibiotics, as these possess an extraordinarily thick peptidoglycan layer that the antibiotic cannot penetrate, and resistances against macrolides, chloramphenicol and tetracyclines (Table 1.2).

(c) alteration of antibiotic target site or substitution of target enzyme (function duplication). This is the mechanism of high-level resistance against $\beta$-lactams, which have low affinity towards certain types of PBPs, against glycopeptides, where a small modification of the cell-wall disaccharide-pentapeptide involves a 1000-fold reduction of binding affinity and against several protein synthesis inhibitors, where a mutation in the ribosome or in the inhibited enzyme renders them inactive (Table 1.2).

(d) increased target concentration or repair of damaged target. Overexpressing the inhibited enzyme is the means of numerous low-level resistances, while repair is generally responsible for resistances against DNA-methylating agents.

\section{Ways of acquiring resistance}

There are several means of sharing genetic information in the microbial world, some of which are routinely used in the laboratory. Bacteria exploit all existing ways of genetic transfer to obtain the necessary genes, as these are essential for survival. In general, the exchange of genetic information can take place by transformation (exchange of DNA), transduction (bacteriophage) or conjugation by plasmids (extrachromosomal DNA), from which the latter is probably the most common (Schaberg et al., 1981; Milatovic \& Braveny, 1987). In addition to conjugative plasmids, bacteria possess transposons that are able to move 


\begin{tabular}{|c|c|c|}
\hline Antibiotic group & Resistance class & Mechanism \\
\hline \multirow{3}{*}{$\beta$-lactams } & inactivation & Hydrolysis by $\beta$-lactamases \\
\hline & target alteration & Reduced affinity PBPs for $\beta$-lactams \\
\hline & sequestration & Suppression of porin expression \\
\hline Glycopeptides & target alteration & $\begin{array}{l}\text { Replacement of D-Ala-D-Ala with } \\
\text { D-Ala-D-Lac or D-Ala-D-Ser }\end{array}$ \\
\hline \multirow{2}{*}{ Aminoglycosides } & inactivation & $\begin{array}{l}\text { Antibiotic acetylation, } \\
\text { phosphorylation or adenylation }\end{array}$ \\
\hline & sequestration & Solid cell wall \\
\hline \multirow{2}{*}{ Tetracyclines } & target alteration & Ribosome conformation change \\
\hline & efflux & Active removal from cytosol \\
\hline Quinolones & target alteration & Mutations in topoisomerase/gyrase \\
\hline \multirow{3}{*}{ MLS-antibiotics } & inactivation & $\begin{array}{l}\text { Antibiotic acetylation, methylation } \\
\text { or hydrolysis }\end{array}$ \\
\hline & target alteration & $\begin{array}{l}\text { Methylation of an adenine in } 23 \mathrm{~S} \\
\text { rRNA of } 50 \mathrm{~S} \text { ribosomal subunit }\end{array}$ \\
\hline & efflux & Active removal from cytosol \\
\hline Chloramphenicol & inactivation & Antibiotic acetylation \\
\hline Sulfonamides & function duplication & $\begin{array}{l}\text { Acquisition of a new enzyme not } \\
\text { susceptible to the drug }\end{array}$ \\
\hline
\end{tabular}

\section{Table 1.2}

Resistance mechanisms against antibiotics.

between transmissible plasmids and chromosomes. These mechanisms also facilitate the spread of resistance genes among different species (Levy, 1992).

\section{Spread of resistance genes in the community}

It is now generally accepted that the spread of antibiotic resistance was initiated and driven by widespread and imprudent use of antibiotics (Neu, 1992; Levy, 2002). As a result, the resistance genes are spread over the world and it is only a question of time when they are transferred into pathogens. The following factors are believed to have an impact on the dissemination of resistance genes (Klare et al., 2003):

(a) Use of antibiotics as growth promoters in animal husbandry. As antibiotics employed for this purpose are relatives of those commonly used in human medicine and the dose they are given is well below the lethal concentration, bacteria that inhabit the gastrointestinal tract were placed among optimal conditions for developing resistance. As a result, the species Enterococcus faecalis and Enterococcus faecium have acquired broad-spectrum resistance against antibiotics. Although these bacteria 
possess a low intrinsic pathogenicity and virulence potential, meat products manufactured from animals treated with antibiotics are often contaminated and consumption resulted in widespread occurrence of these bacteria in the human population, which facilitates the transfer of these genes to pathogens with higher virulence (Wegener et al., 1999; Witte et al., 2000).

(b) Improper use of antibiotics in human medicine. Antibiotics are not freely available in civilised countries, but this obviously does not seem to guarantee prudent use. It has been demonstrated several times that medical doctors prescribe antibiotics even when a viral infection has been diagnosed, and therefore the control over antibiotic circulation is only apparent. Patients often do not follow the directions received and stop taking the drug after they feel better, which may result in the emergence of resistant bacterial strains (Amidi et al., 1975).

(c) Long persistence of antibiotics in the environment. There are relatively few mechanisms that can inactivate antibiotics and after being excreted by individuals or animals, they continue their existence in municipal waters and exert their selective pressure further (Levy, 2001).

(d) Increased use of antimicrobial agents (biocides) in everyday life. Biocides have been introduced relatively recently into household use in forms ranging from floor cleaners, dishwashing detergents and antimicrobial soaps. Although these agents are not structurally related to antibiotics, there are cases reported where resistance to one of these agents resulted in a cross-resistance against antibiotics used in medical praxis. It is currently debated in the literature whether or not their increasing use is responsible for the development of antibiotic resistance (Russel, 2003).

(e) Spread of resistant microorganisms by individuals. We unwarily contaminate our environment with bacteria present on our skin that can then be picked up from the infected surfaces by others. Increased circulation of goods contributes to worldwide spread of resistance (Miller et al., 1996).

\section{Strategies in overcoming bacterial resistance}

Since resistant strains exist against a growing number of commercially available antibiotics, the question of controlling bacterial infections is raised with more emphasis than ever before. It now seems certain that the development of new antibiotics is not sustainable on the longer run and a different attitude towards the microbial world is needed in finding a stable solution. In the meantime, however, treatments should be developed to handle existing 
health hazards (Levy, 2002). Novel trends in combatting bacterial infections can be summarised as follows.

(a) Probiotics. Resistant strains could be replaced by viable, harmless microbes that can successfully compete with pathogens. Bacterial strains should be selected that constitute no risk to human health and be administered to patients. The method can also be successfully employed in preventing infection by establishing and maintaining a competitive flora (Gorbach, 1997; Bengmark, 2002).

(b) Blocking resistance mechanisms. The molecular basis of antibiotic resistance can be determined and new drugs targeting the resistance mechanisms or ones not susceptible to those can be developed. This approach can restore the efficacy of whole families of antibiotics (Nelson \& Levy, 1999).

(c) New drugs. There are several essential steps in the biosynthetic apparatus of bacteria that can be targeted. This approach is essential to treat infections that are not susceptible to existing antibiotics (Bugg \& Walsh, 1992; Di Guilmi et al., 2002).

(d) Implementation of a prudent antibiotic use in the community. Antibiotic resistance is chiefly supported by improper use of antibiotics and work is needed in educating people about the appropriate use of these important drugs. This movement is currently being organised by the Alliance for the Prudent Use of Antibiotics (APUA, www.apua.org).

(e) Vaccine development.

In spite of every effort, a quick solution in overcoming antibiotic resistance is not expected. The resistance genes are spread over the world and it is not certain whether antibiotic resistance can be made reversible. Resistance mechanisms that do not affect biological fitness of bacteria is likely to remain a problem for a prolonged period (Andersson, 2003). 


\section{ANTIVIRAL AGENTS}

The established way of preventing and handling viral infections is undoubtedly the use of vaccines. Vaccines are cheap, safe, often provide lifelong protection and their administration usually does not require expert personnel. Certain viruses, however, exhibit quick alteration of their surface proteins so that they are no longer recognised by the immune system as antigens, and therefore the production of an efficient vaccine is hindered (Stratov et al., 2004). In these cases, antiviral chemotherapy is put into action.

Although the first antiviral drug has been approved as early as 1966 (Davies et al., 1964), new antiviral agents are still inferior even to early antibiotics when the inhibitory efficiency and spectrum of use are compared. Despite considerable development that occurred in this field, antiviral chemotherapy is usually limited to partial inhibition of virus particles and has not yet achieved complete eradication of the infection alone. Significant repression of virus count can generally be achieved and the remainder of viruses is either eliminated or placed back under control by the immune system.

Developers of antiviral agents face unique difficulties in the course of this procedure. As free viruses do not possess operational biosynthetic machinery, inhibitory agents can influence them to a lesser extent only until they infect a cell. After infection, however, viral biosynthetic reactions are so highly integrated into the cellular facility that it proves demanding to inhibit one without interfering with the other. On the other hand, the desired high specificity implies a relatively narrow antiviral profile. An additional complication may be the relatively high variability of viral genotypes. A suitable mutation may confer resistance against the antiviral agent before actually administering the drug and prolonged exposure would select the resistant variant even if its biological fitness were impaired.

\section{Major targets in antiviral action}

Although the details are virus-specific, all viruses infect via a common mechanism that offers the following points of action for antivirals agents:

(a) Cell-free virus.

(b) Attachment and receptor binding.

(c) Membrane fusion (enveloped viruses)/injection of genetic material (naked viruses).

(d) Transcription and integration of genetic material. 
(e) Translation of genetic material.

(f) Processing of expressed proteins and virus assembly.

\section{Antivirals against HIV}

The human immunodeficiency virus (HIV) belongs to the enveloped retrovirus family and was identified as the cause of AIDS in 1984. Globally, AIDS is now the fourth leading cause of death (Omobosola \& Henry, 2003). Driven by the spread of AIDS and the growing number of HIV-infections, several antiviral agents have been developed and introduced into the market (Beach, 1998). Although current drug targets are confined to HIV reverse transcriptase and HIV protease, new drugs in clinical trials offer novel targets and are expected to ease the treatment of resistant strains (Gulick, 2003).

\section{Proteins involved in HIV infection}

The free virus is drifting in the blood stream (Figure 1.5) and recognises vulnerable CD4+ T-cells by a region on the cell surface that is complementary in the charge distribution to viral envelope glycoproteins (Moulard et al., 2000). This region also helps in orienting the virus in the vicinity of a CD4 cellular receptor, which binds the gp120 viral protein with high affinity (Kwong et al., 1998; Rizzuto et al., 1998; Wyatt \& Sodroski, 1998). After receptor binding, gp120 undergoes a conformational change that activates a surface on gp120 to bind the cellular co-receptors CCR5 and CXCR4 (Alkhatib et al., 1996; Dragic et al., 1996; Berger, 1997). This event initiates another conformational rearrangement and exposes the gp41 viral glycoprotein, which in turn promotes fusion of the cellular and viral membranes (Chan et al., 1997). The virus entry is thus completed and the virion is disassembled to functional parts (uncoating).

A large nucleoprotein complex is then introduced into the cytoplasm, which contains two copies of the viral RNA and three viral enzymes essential to promote the infection. These three enzymes comprise the HIV reverse transcriptase, the HIV integrase and the HIV protease, all being promising targets of antiretroviral chemotherapy. The reverse transcriptase consists of two subunits and catalyses the RNA-directed DNA synthesis followed by degradation of the RNA-portion of the DNA-RNA hybrid and then the synthesis of a complementary DNA-chain in order to build up a double helix (Jacobo-Molina et al., 1993; Katz \& Skalka, 1994; Frankel \& Young, 1998). When the synthesis of the viral DNA is ready, it is transferred to the nucleus where it is integrated into host DNA catalysed by the integrase enzyme (Pluymers et al., 2001). Initially, the expression of viral proteins proceeds with a low 


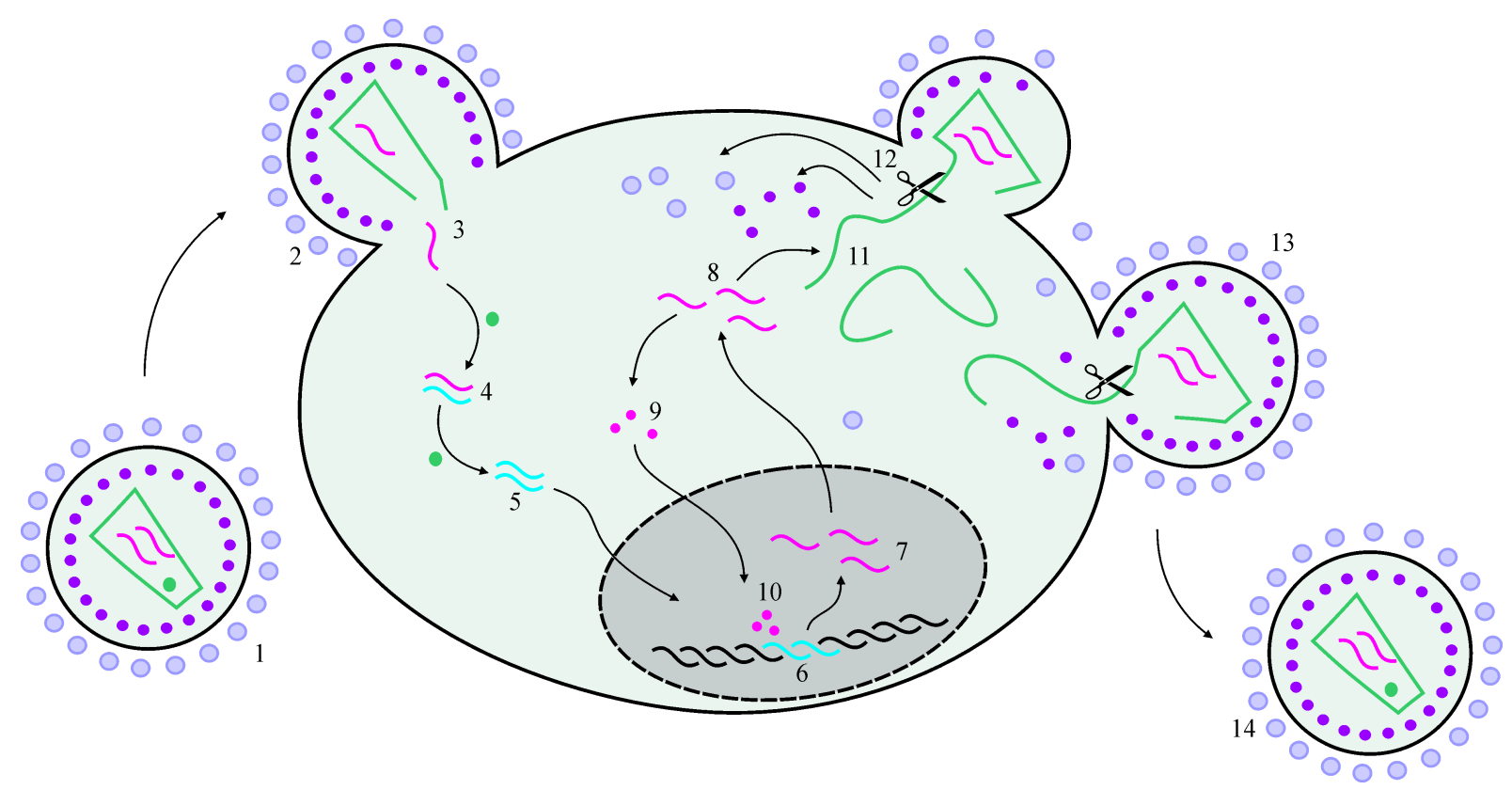

\section{Figure 1.5}

Course of HIV infection. 1: cell-free virus, 2: attachement/entry, 3: uncoating, 4: reverse transcription - RNA/DNA hybrid, 5: double-stranded DNA, 6: nuclear import/integration, 7: transcription of viral genome, 8: viral genomic RNA, 9: translation of viral regulatory proteins, 10: enhanced transcription of viral genome, 11: translation of viral polyproteins, 12: processing of viral polyproteins by HIV protease, formation of matrix, capsid and nucleocapsid proteins, 13: assembly/maturation, 14: assembled infectious virion.

rate, though, and therefore viral regulatory proteins are expressed first that enhance expression and initiate translation of the viral polyprotein complexes (Cullen, 1995). The expressed precursor proteins join the transcribed genomic RNA and form an inactive virion, which is post-processed by the HIV protease by cleaving the polyproteins at specific sites (Nagy et al., 1994). The particle is then surrounded by a modified cell membrane and leaves the cell.

\section{Reverse transcriptase inhibitors}

This group of antiretroviral drugs has been launched by the introduction of zidovudine (Figure 1.6) in 1987 and still comprises the most important drugs for HIV suppression (Table 1.3). Nowadays several members are available and based on the chemical structure three distinct groups can be distinguished: nucleoside analogues, nucleotide analogues and nonnucleoside inhibitors (Beach, 1998). Albeit chemically different, these drugs inhibit the replication of the virus, therefore they prevent new infections, but do not influence virus 


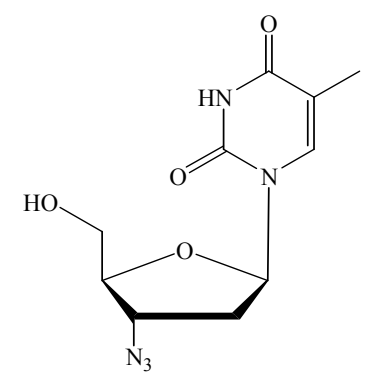

\section{Figure 1.6}

The structure of the first anti-HIV drug, zidovudine. The structure emulates a nucleoside, and is active as the triphosphate derivative. The drug is converted into the active form intracellularly by thymidine kinase. As there is no 3'-phosphate, incorporation of zidovudine results in chain termination. Although zidovudine has an appreciably higher affinity towards the HIV reverse transcriptase than towards human DNA-polymerase, it is toxic to cells where DNA-synthesis occurs.

production in already infected cells. Nucleoside and nucleotide analogues act by being a substrate of the reverse transcriptase enzyme, but cause chain termination upon incorporation since they lack the 3'-OH group. Nonnucleoside inhibitors consist of a structurally diverse group and exert their inhibitory action by binding various areas on the enzyme (Frank, 2002).

\section{Protease inhibitors}

Introduced in 1996, protease inhibitors are important constituents of highly active antiretroviral therapy since they inhibit a complementary step in the virus life cycle, namely the virus assembly (Table 1.3). Their inhibitory action causes release of immature, noninfectious virions and thus protects uninfected cells (Kitchen et al., 1995). Protease inhibitors are transition-state analogues of the substrate of HIV protease (Figure 1.7), which cleaves preferentially between a phenylalanine/tyrosine and a proline residue (Wlodawer, 1994).

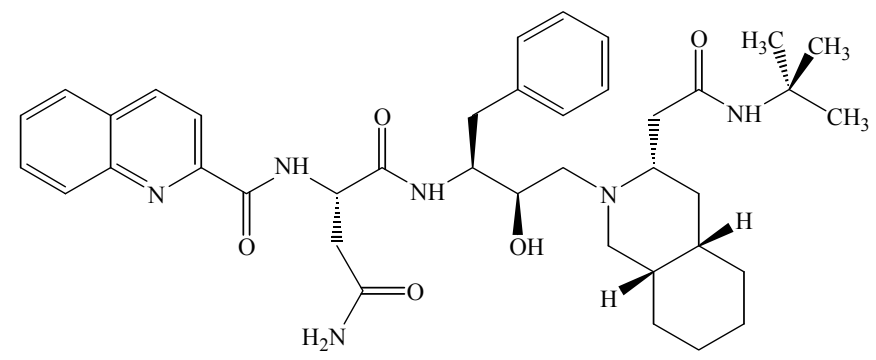

Figure 1.7

Structure of the HIV protease inhibitor saquinavir. The inhibitor emulates the structure of the transition-state intermediate for HIV protease, which cleaves preferentially between a phenylalanine/tyrosine and a proline residue. 


\begin{tabular}{ccc}
\hline Name & Trade name & Introduction \\
\hline Nucleoside/nucleotide analogue reverse transcriptase & inhibitors \\
Zidovudine & Retrovir & 1987 \\
Didanosine & Videx & 1991 \\
Zalcitabin & Hivid & 1992 \\
Stavudine & Zerit & 1994 \\
Lamivudine & Epivir & 1995 \\
Abacavir & Ziagen & 1999 \\
Tenofovir & Viread & 2002 \\
Nonnucleoside reverse transcriptase inhibitors & \\
Nevirapine & Viramune & 1996 \\
Delavirdine & Rescriptor & 1997 \\
Efavirenz & Sustiva & 1998 \\
Protease inhibitors & & \\
Saquinavir & Invirase & 1996 \\
Ritonavir & Norvir & 1996 \\
Indinavir & Crixivan & 1996 \\
Nelfinavir & Viracept & 1997 \\
Amprenavir & Agenerase & 1999 \\
Lopinavir & Kaletra & 2000 \\
\hline
\end{tabular}

Table 1.3

Antiretroviral agents currently used in chemotherapy against HIV.

\section{Promising novel drugs}

Currently, there are several HIV-inhibitors in various clinical phase trials (Pani et al., 2002). Carrageenan, a large polysaccharide isolated from seaweed, is able to bind the virus envelope through its charged sulfate groups and may be used in preventing virus transmission. There are several compounds that target the viral gp120 protein and hinder its binding to the CD4 receptors or to the CCR5/CXCR4 co-receptors. Functional membrane fusion inhibitors are about to be introduced into medical praxis. New compounds that act at the previously untargeted HIV integrase are also actively developed. 


\section{HIV drug resistance}

The HIV reverse transcriptase is not a very specific enzyme and the fidelity of the process is relatively low when compared to other DNA or RNA-polymerases. On average, when creating a reverse transcript, one mutation is introduced (Mansky \& Temin, 1995). As the replication cycle does not take longer than a day, each possible single mutant and several of the double mutants are generated within 24 hours taking into account the number of infected cells and length of the HIV genome. However, it was found that the wild-type virus possesses a higher degree of biological fitness and the mutant viruses are suppressed since their replication rate is slower (Frank, 2002; Omobosola \& Henry, 2003). On the other hand, a suitable mutation may confer resistance to certain inhibitor molecules, and when antiretroviral chemotherapy is initiated, the resistant strain is selected.

\section{Strategies in antiretroviral chemotherapy}

Most of the inhibitors present in clinical practice require multiple mutations until resistance is developed (Balint, 2001); moreover, in certain cases a mutation induced by one inhibitor makes the virus susceptible again to another inhibitor, against which it has already acquired resistance (Larder, 1995). Nevertheless, the development of resistance is usually fast and takes between a week and a year when the drug is given in monotherapy (Beach, 1998), depending on the number of mutations that have to accumulate. For that reason, it is now generally accepted that anti-HIV chemotherapy should be conducted by employing a minimum of three drugs in parallel, at least two of which are reverse transcriptase inhibitors (Frank, 2002). When the virus is not allowed to replicate, new mutations do not occur and thus resistance cannot develop. Adherence to drug regimen is also an important factor; studies suggest that omitting as low as $5 \%$ of the medicament doses increases considerably the risk of losing control over the virus (Paterson et al., 2000). As pill-taking imposes an enormous burden on patients, suboptimal adherence is not uncommon and an often cause of failure. Assessment of adherence is an important factor in deciding when to initiate the treatment (Frank, 2002; Omobosola \& Henry, 2003).

\section{Facing resistance}

If resistance develops, one option may be to remove the given drug from the regimen and wait for the wild-type, sensitive virus to reemerge (Omobosola \& Henry, 2003). However, some mutations do not significantly affect biological fitness of the virus and the mutation will persist. As certain mutations induce cross-resistance to several other inhibitors, 
the emergence of a resistant strain complicates the treatment substantially. There is also a growing incidence of transmitting resistant viral strains, which exacerbates the future prospects of HIV therapy (Little et al., 1999).

The development of resistance during antiretroviral therapy will sooner or later occur. Since more inhibitors are used in parallel, the resistance against a certain drug may not become manifested, but the chances of developing resistance against the remaining drugs are enhanced. For that reason, resistance testing is an important supplement to the drug regimen. In the case of virologic failure, a resistance test is performed to determine which drugs have retained their activity (Sebastian \& Faruki, 2004).

A further complication of HIV is the potency of causing latent infection. The reservoir of HIV-infected cells in the body is not supposed to be cleared out in a short time and the therapy needs to be continued for decades. For this reason, HIV is considered incurable and sustainability of anti-HIV chemotherapy is dependent upon the continuous development of new antiviral agents. 



\section{METHOdS IN CRYSTALLOGRAPHY}

\section{INTRODUCTION}

The micro world has attracted the attention of mankind for a long time, but the actual deepness of examination was limited by the level of development. First investigations were most likely to be performed by eye, and later were supported by lens in form of a magnifying glass or microscope. However, as the size of the resolvable details is in the order of the wavelength of radiation used for the examination, the atomic structure of molecules cannot be seen directly through an optical device with visible light, although visible light can be used in spectroscopic techniques to obtain geometric information of the molecule. It turns out that average interatomic distances lie in the wavelength range of soft $\mathrm{X}$-ray radiation, which would therefore be suitable for such an investigation, but the refractive index of nearly all materials for X-rays is very near to one, which implies that a refocused image of the object cannot be obtained.

Diffraction techniques differ from conventional microscopy in that they detect the primary diffraction image and "refocusing" is performed mathematically. X-ray diffraction can therefore be applied to determine the structure of molecules. However, as the interaction of X-rays with matter is relatively weak, the presence of a vast number of molecules is necessary to get diffraction above the level of detection. This can be achieved by using an amorphous solid or in gas phase, where the molecules are in all possible orientations, or with a crystal, where a strict ordering is present. In the following only the crystal will be discussed in detail.

Let us now consider the scattering that occurs when a single molecule is irradiated by monochromatic radiation. The molecule is supposed to consist of atoms $i$ with position vectors $\mathbf{r}_{i}$ from a suitable origin, let the wavelength be denoted by $\lambda$, and define the scattering vector $\mathbf{S}$ as $\mathbf{S}=\mathbf{s}-\mathbf{s}_{\mathbf{0}}$, where $\mathbf{s}_{\mathbf{0}}$ and $\mathbf{s}$ give the direction of the primary X-ray beam and any diffraction direction, respectively, and their length is by definition $1 / \lambda$. Then the function $\mathbf{F}(\mathbf{S})$ can be calculated as:

$$
\mathbf{F}(\mathbf{S})=\sum_{i} f_{i}(\mathbf{S} \mid) \exp \left(2 \pi i \mathbf{r}_{i} \mathbf{S}\right)
$$


where $f_{i}(|\mathbf{S}|)$ is the scattering factor of atom $i$, and depends only on the length of $\mathbf{S}$, which can be given as $\sin \Theta / \lambda$, where $2 \Theta$ is the scattering angle. The measurable scattered intensity in direction s would then be proportional to $|\mathbf{F}(\mathbf{S})|^{2}$. However, this does not correspond to any of the scenes mentioned above, as in the first case the measured intensity distribution is a sum of scattering from all molecules, each in an individual orientation, while in case of a crystal, the convolution of scattering from one molecule with the scattering from the lattice can be measured, and can also be described as $\mathbf{F}(\mathbf{S})$ sampled on a grid determined by lattice parameters. The originally continuous $\mathbf{F}(\mathbf{S})$ is thus converted into a discrete function, which has the same value as $\mathbf{F}(\mathbf{S})$ if $\mathbf{S}$ can be expressed as

$$
\mathbf{S}=h \mathbf{a}^{*}+k \mathbf{b}^{*}+l \mathbf{c}^{*}
$$

where $\mathbf{a}^{*}, \mathbf{b}^{*}$ and $\mathbf{c}^{*}$ are basis vectors of the reciprocal lattice and $h, k$ and $l$ are integer numbers, and zero elsewhere. The reciprocal unit cell is defined as

$$
\mathbf{a a}^{*}=1 \text { and } \mathbf{a}^{*} \mathbf{b}=\mathbf{a} * \mathbf{c}=0
$$

where $\mathbf{a}, \mathbf{b}$ and $\mathbf{c}$ constitute the direct-space unit cell, and analogously for all three vectors. The scattered intensity in direction $\mathbf{s}$ is proportional to $|\mathbf{F}(\mathbf{S})|^{2}$, which can therefore easily be measured; phases of certain structure factor combinations can also be measured, but only with great difficulty and therefore it is not practised. Thus, half of the information necessary to "refocus" the diffraction image is lost in the measurement stage: to reconstruct $\mathbf{F}(\mathbf{S})$ as a complex number, both the amplitude and the phase are necessary. This is the crystallographic phase problem, and since it requires simultaneous phase acquisition for all reflections, it appears to be difficult to solve.

The process of X-ray crystallographic structure determination of a molecule consists of several steps: (i) crystallisation, (ii) measurement, (iii) solution of the phase problem, (iv) generation of the atomic model (model building) and (v) fitting the atomic model to the measured data (refinement).

\section{DATA COLLECTION AND PROCESSING}

Biological crystallography, in accordance to its scientific character, utilizes primary data that originate usually from a measurement. The measured data can then be analysed using various methods, but the precision of the end result is strongly dependent on the accuracy of the starting point. The measurement is therefore a crucial step in the structure determination process. 
The raw data measured with an area detector consist of images that display the intensity distribution in the measured directions. This distribution is composed of two parts:

(a) Reflections occur in directions for which the Bragg-conditions hold (i.e. $\mathbf{S}$ can be expressed as a linear combination of the three reciprocal lattice vectors with integral coefficients) and $|\mathbf{F}(\mathbf{S})|$ is not too small.

(b) Background scattering coming from the air, the crystal support, etc.

These two can easily be discriminated because Bragg-scattering is a discrete function, while background scattering is continuous. The information present on these images can be divided into two classes: the positions of the Bragg-reflections and their intensities.

\section{Indexing}

The crystal is usually mounted in a random orientation and in the general case, nothing is known about it. In order to be able to predict the positions of Bragg reflections, one should first determine the unit cell dimensions of the crystal and the orientation of these with respect to the laboratory coordinate system. This is equivalent to assigning indices $h, k, l$ (or $\mathbf{h}$ in vector notation) to all reflections and this process is thus called indexing.

To establish the lattice periodicity, the diffraction images are peak-searched to determine observed positions for the reflections. The centroids of spots are determined in two dimensions (if thin sliced data are available, also in the third, rotation direction), and converted from the laboratory coordinate system to crystal-fixed coordinates. It is crucial to obtain as accurate coordinates as possible, and it is also important to exclude any contamination originating from background scattering or electronic noise; usually only strong spots are included in the reflection list and spurious reflections, consisting of a single pixel or being too close to other spots are filtered out. These observed scattering vectors $\mathbf{s}_{i}$ can then be transformed into a crystal-fixed coordinate system. Transformation from the laboratory coordinate system requires precise knowledge of the direct beam coordinates and detector distortion parameters, which should therefore be input adequately (Figure 2.1).

Given the availability of a sufficient number of spots representative of the lattice periodicity $\left(\mathbf{x}_{i}=\mathbf{s}_{i}-\mathbf{s}_{0}\right.$ vectors, where $\left.i=1, \ldots, n\right)$, indexing can be performed using several algorithms. The task is to determine a matrix $\mathbf{A}$ for that

$$
\mathbf{x}_{i}=\mathbf{A} \mathbf{h}_{i}
$$



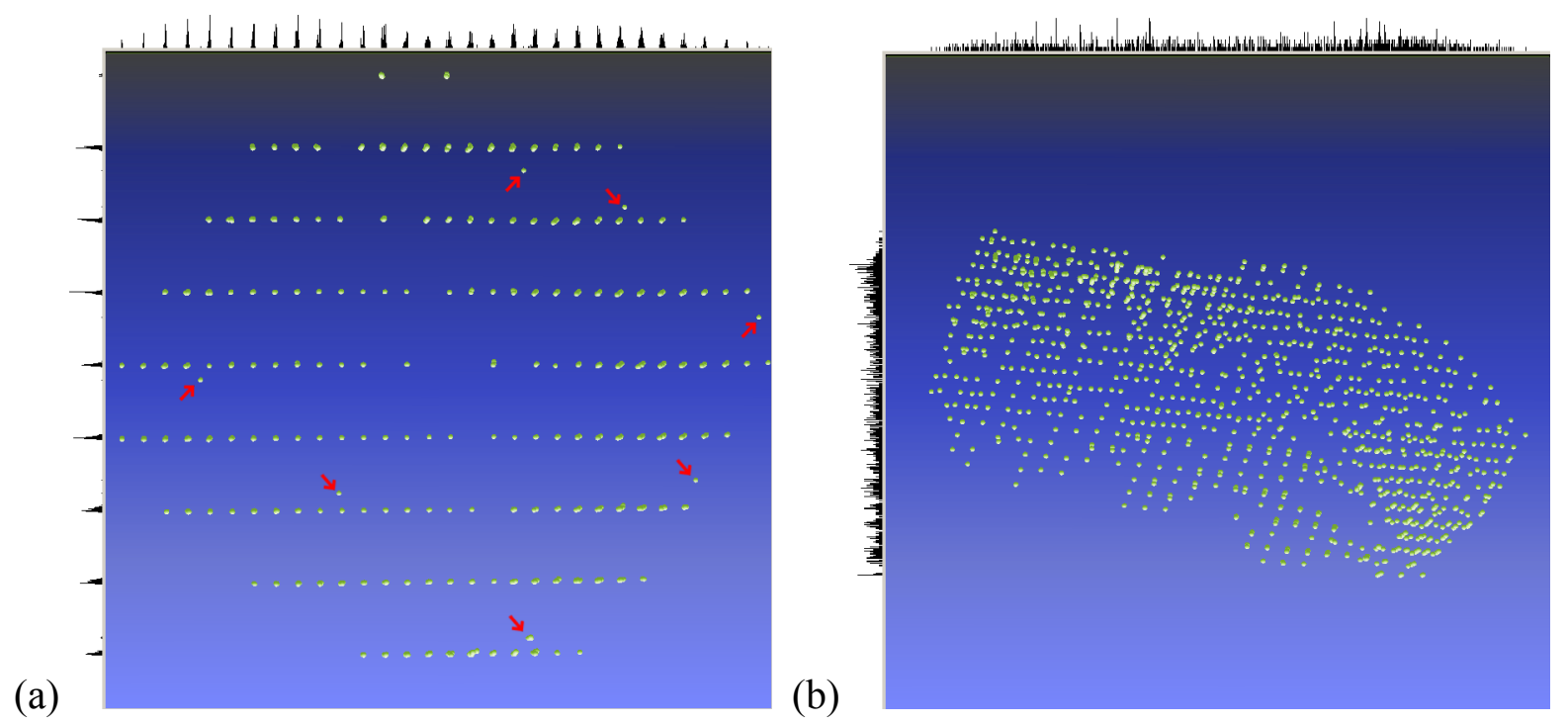

Figure 2.1

Observed reflection centroids displayed in crystal-fixed coordinate system. In (a) the lattice is oriented so that the view is along one real-space axis. The reflections form lines and cluster at certain positions (see projections on top and left). Few noise reflections (marked with red arrows) are easily identified, but they do not affect the projection seriously and their effect is marginal. Imprecise detector parameters would lead to curved lines and broad clusters and would complicate indexing. In (b) the same lattice is shown, but in a random orientation.

is fulfilled with the components of $\mathbf{h}_{i}$ being close to integer numbers for most of the reflections. The matrix A describes the unit cell vectors and their orientation with respect to the crystal coordinate system and can be written:

$$
\mathbf{A}=\left[\begin{array}{lll}
a_{11} & a_{12} & a_{13} \\
a_{21} & a_{22} & a_{23} \\
a_{31} & a_{32} & a_{33}
\end{array}\right]
$$

the components of which are related to the reciprocal unit cell as

$$
\left|\mathbf{a}^{*}\right|^{2}=a_{11}^{2}+a_{12}^{2}+a_{13}^{2} \quad\left|\mathbf{b}^{*}\right| \mathbf{c}^{*} \mid \cos \alpha^{*}=a_{12} a_{13}+a_{22} a_{23}+a_{32} a_{33}
$$

and similarly for the remaining unit cell constants.

\section{The Sparks algorithm}

The three shortest non-coplanar vectors are chosen from the list of vectors and the matrix $\mathbf{X}$ is composed from them (Sparks, 1976). Rewriting equation (4) and rearranging by taking inverses the following is obtained:

$$
\mathbf{A}^{-1}=\mathbf{H X}^{-1}
$$


which can also be written as:

$$
\mathbf{a}_{1}=\mathbf{g}_{1} \mathbf{X}^{-1} \quad \mathbf{a}_{2}=\mathbf{g}_{2} \mathbf{X}^{-1} \quad \mathbf{a}_{3}=\mathbf{g}_{3} \mathbf{X}^{-1}
$$

where $\mathbf{g}_{1}, \mathbf{g}_{2}, \mathbf{g}_{3}$ are row vectors composed of integer numbers. The algorithm proceeds by generating integer triples starting with small numbers and solves one of equations (8) for $\mathbf{a}_{1}$, $\mathbf{a}_{2}$ or $\mathbf{a}_{3}$. A solution is accepted if for most of the observed reciprocal vectors holds

$$
\mathbf{a}_{1} \mathbf{x}_{i}=h_{i} \quad i=1, \ldots, n
$$

where $h_{i}$ is an integer, and analogously for $\mathbf{a}_{2}$ and $\mathbf{a}_{3}$. The shortest non-coplanar vectors are selected from the generated list and cell reduction is performed. This algorithm was designed to find the unit cell from a small number of reflections (ca. 25) and then checking the axial directions to assure that not a real-space subcell has been selected. The method is very quick, but sensitive to noise reflections or reflections from satellite crystals; the three selected vectors have to belong to the same lattice. An extension of the method uses difference vectors calculated from the reflection list as:

$$
\mathbf{x}_{i j}=\mathbf{x}_{i}-\mathbf{x}_{j} \quad i, j=1, \ldots, n
$$

which have a higher chance of being elementary unit cell vectors and also difference vector clusters with many nearly identical members assure that the vector is likely to correspond to a single lattice.

This algorithm can be modified to index overlapped lattices. However, the chance for selecting vectors that do not belong to one lattice is much higher in the presence of a twin component. This is overcome by difference-vector cluster analysis and by increasing the number of trials.

\section{The Kabsch algorithm}

Kabsch developed a method that depends on the availability of many reflections, as is usually the case for biological samples and area detectors (Kabsch, 1993). This algorithm starts with the generation of low-resolution difference vectors, which are analysed on a threedimensional histogram. The histogram is peak-searched and a list of difference-vector clusters are obtained with length $\mathbf{v}_{i}{ }^{*}$ and population $f_{i}(i=1, \ldots, m)$. In the next step, the set of noncoplanar $\mathbf{b}_{1}{ }^{*}, \mathbf{b}_{2}{ }^{*}, \mathbf{b}_{3}{ }^{*}$ vectors are selected from the list that maximise the function $Q$ (defined as follows) and taken as the reciprocal basis vectors.

$$
Q\left(\mathbf{b}_{1}^{*}, \mathbf{b}_{2}^{*}, \mathbf{b}_{3}^{*}\right)=\sum_{i=1}^{m} f_{i} \exp \left(-\sum_{k=1}^{3}\left\{\left[\max \left(\left|\xi_{k}^{i}-h_{k}^{i}\right|-\varepsilon, 0\right) / \varepsilon\right]^{2}+\left[\max \left(\left|h_{k}^{i}\right|-\delta, 0\right)\right]^{2}\right\}\right)
$$

where $\varepsilon$ and $\delta$ are suitably chosen constants, $\xi_{k}^{i}=\mathbf{v}_{i}{ }_{i} \mathbf{b}_{k}, h_{k}{ }_{k}$ is the nearest integer to $\xi_{k}^{i}$ and $\mathbf{b}_{k}(k=1, \ldots, 3)$ is the corresponding real-space axis as defined in (3). This function penalises a 
basis vector set if there are many $\mathbf{v}_{i}{ }^{*}$ difference vector clusters with several members that deviate in indices from integer numbers by more than $\varepsilon$, or if these low-resolution difference vectors happen to have indices higher than $\delta$ (prevents the selection of too short basis vectors that may occur in the presence of a twin lattice). This basis set is then refined using difference vector clusters $\mathbf{v}_{i}{ }^{*}$ and the reduced cell is determined. Indexing of observed vectors $\mathbf{x}_{i}$ is performed by a local indexing algorithm that indexes vectors with respect to reflections with previously determined indices. This algorithm performs well in most cases, but requires many reflections. It is not sensitive to errors in detector distortion parameters and may index twin lattices in favourable cases.

\section{The Duisenberg algorithm}

This has been specially devised for problem cases, i.e. twin lattices (Duisenberg, 1992). Three reflections are chosen randomly from the reflection list and the normal of their plane is calculated. A projection of all reflections is then calculated onto this direction. If the three reflections belong to the same lattice, their plane normal coincides with a direct-lattice vector and a projection onto this direction would result in clustering at positions corresponding to integer numbers. "Alien" reflections do not obey this rule and land somewhere between the clusters and are discarded (Figure 2.1). The periodicity of the clusters is determined and stored together with the number of fitting reflections. If the triplet contains "alien" reflections (noise or contamination from other twin domains), a very short period or only a few fitting reflections are obtained and therefore can be filtered out. This search is performed with a large number $(>1000)$ of triplets and the direct axes found are combined to index as many reflections as possible in all three dimensions within a predefined deviation from integer values. Non-fitting reflections can be used in a following run to determine lattice parameters for them as well. This algorithm is comparatively slow, but gives appropriate results with few and also with many reflections.

\section{The Fourier algorithm}

In this method each direction of a hemisphere is considered on a suitable grid (Steller et al., 1997). Projections are generated onto the selected direction, and the periodicity is established with a Fourier-transform (the position of largest maximum after the origin peak gives the periodicity and its height corresponds to the number of fitting vectors). Large peaks are therefore likely to indicate potential direct-lattice vectors, which are refined and stored, and at the end three linearly independent vectors are selected and cell reduction is performed. This algorithm is relatively fast but requires many reflections, as for the Fourier- 
transformation to give sharp peaks several lattice plane intersections are needed. However, a small number of "alien" reflections is tolerated well and the algorithm could in principle be used to index twin lattices with a slight modification in the cell selection routine. Another improvement possibility is to perform a three-dimensional Fourier-transformation and determine all three cell dimensions in one step.

\section{The cell_now algorithm}

This program (Bruker Nonius, 2002) generates random direct-space vectors $\mathbf{d}$ between pre-defined limits in length and then for each vector the function

$$
Q=\sum_{i=1}^{n} w_{i}\left(T_{i}-M_{i}\right)^{2} \text { with } w_{i}=\frac{I_{i}^{\frac{1}{2}} P^{2}}{P^{2}+\left(T_{i}-M_{i}\right)^{2}}
$$

is minimised, where $I_{i}$ is the intensity of reflection $i, T_{i}=\mathbf{d} \mathbf{x}_{i}$ and $M_{i}$ is the nearest integer. The weights $w_{i}$ downweight non-fitting reflections that deviate more than $P^{2}$ from integer indices (which is similar to $\varepsilon$ in Kabsch's formula) and thus minimise the effects of "alien" reflections or reflections from another twin domain. After locating the first domain, the program rotates the same unit cell and tries to dock it using non-fitting reflections. Since the cell is only reoriented and not redetermined, minor domains can also be located in this way. The algorithm is relatively slow, but is insensitive to high numbers of noise reflections or the presence of multiple twin domains.

\section{Orientation and cell parameter refinement}

For an accurate intensity measurement of Bragg peaks, the predicted spot positions should coincide with the observed ones preferably within one pixel. Indexing algorithms are not able to achieve such high precision since they disregard errors arising from imprecise alignment. Taking the measurement geometry into account, most of these errors can be corrected for given the availability of an indexed list of reflections. This procedure optimises a limited number of parameters (typically the unit cell dimensions, the orientation matrix, direct beam coordinates and detector distortion parameters) that affect observed or predicted reflection centroids and minimises the deviations generally with a least-squares procedure.

\section{Intensity measurement}

The raw data collected in a diffraction experiment consist of images with showing a characteristic pattern of spots. However, most of the information present on these images do not get used in subsequent procedures, and can be discarded. The only important information 


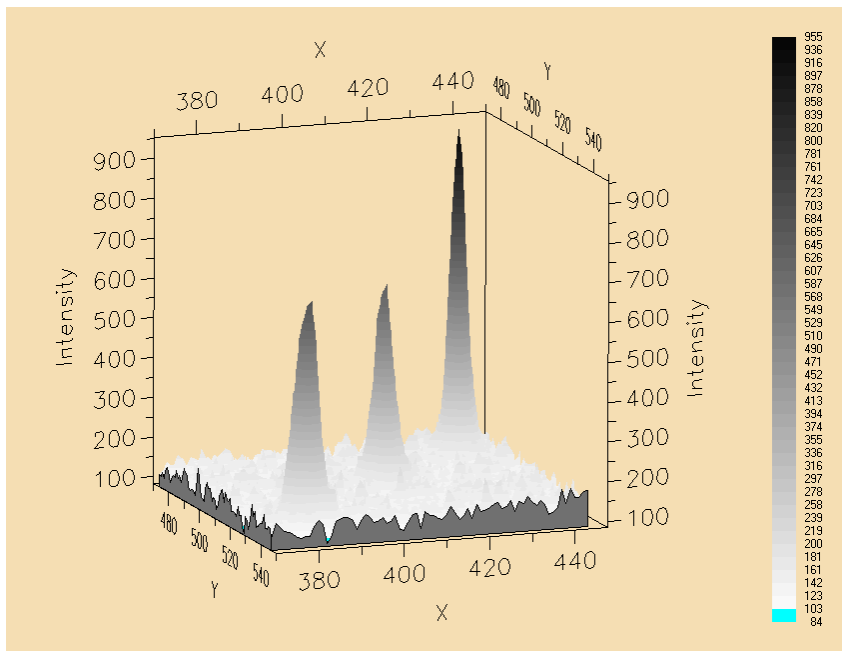

\section{Figure 2.2}

The intensity of reflections is proportional to the volume under their contours after the estimated background has been subtracted. This may be complicated when the background noise is high. Reflection profiles are also slightly different because of the spatial resolution of the detector.

that has to be extracted from the images with as high precision as possible is the intensity of the individual Bragg peaks. Since after this step the information content of the frames (from the crystallographic point of view) is converted into a different form that requires much less storage space without the loss of information, this process is often referred to as data reduction.

The intensity of a reflection is given by the volume under the peak contours in an area detector (Figure 2.2). However, to obtain a precise estimate of the reflection intensity, the effect of background and Poissonian noise has to be taken into account, which is not a trivial procedure considering that the intensities are inherently weak. In the case of area detectors, the observed intensity distribution in a suitably chosen area around a Bragg-peak can be written as:

$$
I_{i}=I_{i}^{p e a k}+I_{i}^{b k g}
$$

where $I_{i}, I_{i}^{\text {peak }}, I_{i}^{\text {bkg }}$ are pixel counts that are observed, attributed to Bragg-scattering or diffuse scattering with unspecified origin, respectively, and $i$ is a pixel within the measurement box. If the pixel $i$ is far from the reflection centroid, the contribution of Bragg-intensity is negligible, so the observed intensity is accounted for by the background. The measurement box can therefore be divided into background $(B)$ and peak $(P)$ regions, with $n$ and $m$ pixels, 
respectively. As the background is believed to be a smoothly varying function, it can be estimated as (Leslie, 1999):

$$
I_{i}^{b k g}=a x_{i}+b y_{i}+c
$$

where $a, b$ and $c$ are constants, $x_{i}$ and $y_{i}$ are the coordinates of pixel $i$ relative to the peak centroid. For thin-slice data collection, a third term dependent on the rotation angle can also be included (Duisenberg et al., 2003). This background plane can be determined by leastsquares fitting using pixels from the background region. An alternative technique in determining the background is to read consecutive frames spanning a defined angular range, average pixel values that are near to the value of the lowest (Kabsch, 2001) and then smooth them with a suitable function.

There are several factors that have to be considered when giving an estimate for the reflection intensity, $I^{\text {peak }}$. As the simplest approach, this can be evaluated by

$$
I^{\text {peak }}=\sum_{i \in P}\left(I_{i}-I_{i}^{b k g}\right)
$$

where $I_{i}^{b k g}$ is estimated by either of the methods described above (summation integration). This is referred to as the BPB (background-peak-background) method, and has the outstanding property that if $I_{l}^{\text {peak }}$ is negligible in the background region, the estimate is safe with regard to systematic error (Diamond, 1969). However, since the standard uncertainty associated with this intensity estimate can be given as

$$
\sigma_{I^{\text {peak }}}^{2}=G\left[\sum_{i \in P} I_{i}+\left(\frac{m}{n}\right)^{2} \sum_{i \in B} I_{i}\right] \approx G\left(I^{\text {peak }}+\frac{m+n}{n} I^{b k g}\right)
$$

where

$$
I^{b k g}=\sum_{i \in P} I_{i}-I^{\text {peak }} \approx \frac{m}{n} \sum_{i \in B} I_{i}
$$

and $G$ is the detector gain (number of X-ray photons/pixel count), the background governs the standard deviation when the intensity of the Bragg-peak is low. The division of the measurement box into peak and background areas is also somewhat arbitrary, but to avoid systematic errors the peak region should not be selected too small. On the other hand, too large a peak region increases the standard deviation and decreases signal-to-noise for weak reflections. There are several approaches for dividing the measurement box into peak and background regions, e.g. the one that maximises $I / \sigma(I)$ (Lehmann \& Larsen, 1979) or to predict the peak area based on physical factors like crystal size or beam divergence (Duisenberg et al., 2003). 
An improvement in the precision of weak intensities can be achieved with profile fitting that estimates the intensity as (Otwinowski \& Minor, 1997)

$$
I^{\text {peak }}=\frac{\sum_{i \in P} \frac{p_{i}\left(I_{i}-I_{i}^{b k g}\right)}{\sigma_{i}^{2}}}{\sum_{i \in P} \frac{p_{i}^{2}}{\sigma_{i}^{2}}}
$$

where $\sigma_{i}^{2}$ is the variance of pixel $i$ and $p_{i}$ is the value of the predicted profile. If the intensity of the peak compared to the background is low, the standard uncertainty of the profile-fitted intensity can be given as

$$
\sigma_{I^{\text {peak }}}^{2} \approx G I_{\text {average }} \frac{\left(\sum_{i \in P} p_{i}\right)^{2}}{\sum_{i \in P} p_{i}^{2}}
$$

with all the pixels in the peak region having approximately the same value ( $\left.I_{\text {average }}\right)$. The expected gain in signal-to-noise ratio can then be estimated (Leslie, 1999)

$$
\frac{\sigma_{I_{\text {summed }}^{\text {peak }}}^{2}}{\sigma_{I_{\text {proflie }}^{\text {peak }}}}=\frac{m \sum_{i \in P} p_{i}^{2}}{\left(\sum_{i \in P} p_{i}\right)^{2}}
$$

Since the value of the right-hand side, which depends on the shape of the standard profile, is typically 2 , the increase in signal-to-noise ratio for very weak reflections is about $40 \%$. It can also be demonstrated that for strong reflections the profile-fitting procedure reduces to summation integration. However, to avoid systematic errors in intensity estimation, the predicted profile $p_{i}$ has to be a good estimate of the observed reflection profile (Diamond, 1969). To ascertain a proper match, profiles are not modelled by any theoretical function, but learnt from the observed profile of strong reflections. The profile changes across the face of the detector, owing to changes in incidence angle and certain geometric factors. For that reason, the profile of a reflection is usually estimated as a weighted average of profiles from strong reflections being spatially close. An alternative approach is the transformation of pixels into a local coordinate system described by Kabsch (2001). Defining the basis vector set as

$$
\begin{aligned}
& \mathbf{a}=\mathbf{n} \times \mathbf{s} /|\mathbf{n} \times \mathbf{s}| \\
& \mathbf{b}=\mathbf{s} \times \mathbf{a} /|\mathbf{s} \times \mathbf{a}| \\
& \mathbf{c}=\left(\mathbf{s}+\mathbf{s}_{\mathbf{0}}\right) /\left|\mathbf{s}+\mathbf{s}_{\mathbf{0}}\right|
\end{aligned}
$$


where $\mathbf{n}$ is any unit vector perpendicular to the incident beam vector $\mathbf{s}_{0}$, the spots are treated as if they had followed the shortest path through the Ewald sphere. This transformation substantially reduces variation in reflection profile, and yields well-conserved threedimensional model profiles applicable to all reflections. Three-dimensional profile fitting eliminates an additional problem, namely that the profile of partially recorded reflections can significantly differ from that of fully recorded reflections and depend on the degree of partiality (Leslie, 1999).

\section{Scaling and post-refinement}

Integrated intensities should be corrected for variations in incident beam intensity, diffraction volume, crystal decomposition, absorption and several other factors. This is done by minimising the deviation of symmetry or Friedel-related reflections by a correction dependent on these physical factors. The corrected intensity can be written as

$$
I_{c}=I_{o} S(n) P(\mathbf{u}) R(n, \Theta)
$$

where $I_{o}$ and $I_{c}$ are the uncorrected and corrected intensity, respectively, $n$ is the frame number, $\mathbf{u}$ represents direction cosines relative to reciprocal unit cell axes and $\Theta$ is the Braggangle. The first term $S(n)$ assigns a scale factor for each frame and corrects for changes in incident beam intensity and in diffraction volume. $P(\mathbf{u})$ describes anisotropic absorption by the crystal and the support and can be estimated as

$$
P(\mathbf{u})=a_{0}+\sum_{l} \sum_{m=-l}^{l} a_{l m} \mathrm{Y}_{l}^{m}(\mathbf{u})
$$

where $a_{0}$ and $a_{l m}$ are coefficients and $\mathrm{Y}_{l}^{m}(\mathbf{u})$ denotes an $l$-order spherical harmonic. The third term $R(n, \Theta)$ corrects for decomposition manifesting as an increase in $B$-values and can be written as

$$
R(n, \Theta)=\exp \left(B(n) \frac{\sin ^{2} \Theta}{\lambda^{2}}\right)
$$

where $B(n)$ gives the variation of temperature factors as a function of frame number, and since it is correlated with scale factors, a smoothly varying function should be used. These equations are valid for fully recorded reflections or for three-dimensional profile fit. The partials can either be added to form a fully recorded reflection or each partial can be used to give an estimate of the total intensity (Rossmann \& van Beek, 1999)

$$
I_{o}^{n}=\frac{I_{n}}{\zeta_{n}}
$$


where $I_{n}$ is the intensity measured on frame $n$ and $\zeta_{n}$ is the partiality, which is defined as the volume portion of the reflection that swept through the Ewald sphere on that frame. The function minimised can be written as

$$
\Psi=\sum_{\mathbf{h}} \sum_{i=1}^{n_{\mathbf{h}}} w_{\mathbf{h}}^{i}\left(I_{c}^{i}(\mathbf{h})-\langle I(\mathbf{h})\rangle\right)
$$

where the inner summation goes for all $n_{\mathbf{h}}$ observations of the unique reflection $\mathbf{h},\langle I(\mathbf{h})>$ is the mean intensity that can be taken as the median of all $I_{c}^{i}(\mathbf{h})$ values or as a weighted average. After the scaling procedure, the agreement of symmetry-related reflections is indicated by

$$
R_{\mathrm{int}}=\frac{\sum_{\mathbf{h}} \sum_{i=1}^{n_{\mathbf{h}}}\left|I_{c}^{i}(\mathbf{h})-\langle I(\mathbf{h})\rangle\right|}{\sum_{\mathbf{h}} \sum_{i=1}^{n_{\mathrm{h}}} I_{c}^{i}(\mathbf{h})}
$$

where only those unique reflections are included in the summation that have more than two symmetry equivalents.

When all the reflections are put on the same scale, outliers (large, but sporadic fluctuations in the data) can be identified and rejected. In the last step, standard uncertainties estimated in the integration stage have to be corrected to explain the observed variance of symmetry equivalent reflections. This is usually achieved by modifying them as given in

$$
\sigma_{a b s}^{2}(\mathbf{h})=K\left[\sigma_{\text {raw }}^{2}(\mathbf{h})+(g\langle I(\mathbf{h})\rangle)^{2}\right]
$$

where $K$ and $g$ are determined such, that the quantity

$$
\chi^{2}=\sum_{\mathbf{h}} \frac{1}{n_{\mathbf{h}}-1} \sum_{i=1}^{n_{\mathbf{h}}} \frac{\left(I_{c}^{i}(\mathbf{h})-\langle I(\mathbf{h})\rangle\right)^{2}}{\sigma_{c}^{2}(\mathbf{h})}
$$

is near to one and does not show any variation neither as a function of resolution nor as a function of intensity.

After the measurement one can obtain the most accurate unit cell by determining the lattice constants that best fit all observed reflection positions. In the case of a threedimensional profile fit, reflection centroids are available and this procedure is identical to initial cell refinement apart from the fact that the values of geometric parameters are better defined. For two-dimensional profile fit procedures, centroid positions can be determined from the estimated partiality, and refinement of partiality therefore allows the determination of mosaicity and lattice parameters (Rossmann \& van Beek, 1999). 


\section{SOLUTION OF THE PHASE PROBLEM}

After data processing, a list of structure factors $|\mathbf{F}(\mathbf{S})|$ is available (expressed as $\left|\mathbf{F}_{\mathbf{h}}\right|$ and the corresponding unit cell). However, to calculate an electron density map, the phase $\varphi_{\mathbf{h}}$ of the complex number

$$
\mathbf{F}_{\mathbf{h}}=\left|\mathbf{F}_{\mathbf{h}}\right| \exp i \varphi_{\mathbf{h}}
$$

is necessary ( $i$ is the imaginary unit). There are several methods available for obtaining approximate values for the phases; an average phase error of $60^{\circ}$ is enough to calculate an interpretable electron density map, or at least to initiate phase improvement.

\section{Direct methods}

At a sufficiently high resolution, the number of unique reflections is much higher than the number of coordinates for atoms present in the asymmetric unit, i.e. the phase problem is overdetermined. Direct methods exploit this fact and are able to provide phase estimates for most of the reflections but only if data extend to high (1.20 A or better) resolution.

\section{The tangent formula}

Unlike the amplitudes, which are well defined apart from a global scale factor, the phases depend on the choice of the origin. It is easily conceivable that an origin shift $\mathbf{0}$ changes the phase values according to

$$
\varphi_{\mathbf{h}}^{\prime}=\varphi_{\mathbf{h}}-2 \pi \mathbf{h o}
$$

However, it can be proved that the product of $n$ structure factors is invariant under origin translation if

$$
\mathbf{h}_{1}+\mathbf{h}_{2}+\ldots+\mathbf{h}_{n}=0
$$

The phases for certain classes of these structure factor combinations (called structure invariants) can be estimated from the reflection intensities under given assumptions. One such assumption is that the electron density is not negative [if $\mathbf{F}(000)$ is included in the summation]; while another approach is to assume the presence of randomly distributed resolved point atoms, which is in practice more effective. To fit this second assumption, the observed structure factors are modified in order to remove the effects of thermal motion and normalised structure factors are introduced as

$$
\left|\mathbf{E}_{\mathbf{h}}\right|^{2}=\frac{\left|\mathbf{F}_{\mathbf{h}}\right|^{2} / \varepsilon}{\left\langle\left|\mathbf{F}_{\mathbf{h}}\right|^{2} / \varepsilon\right\rangle}
$$




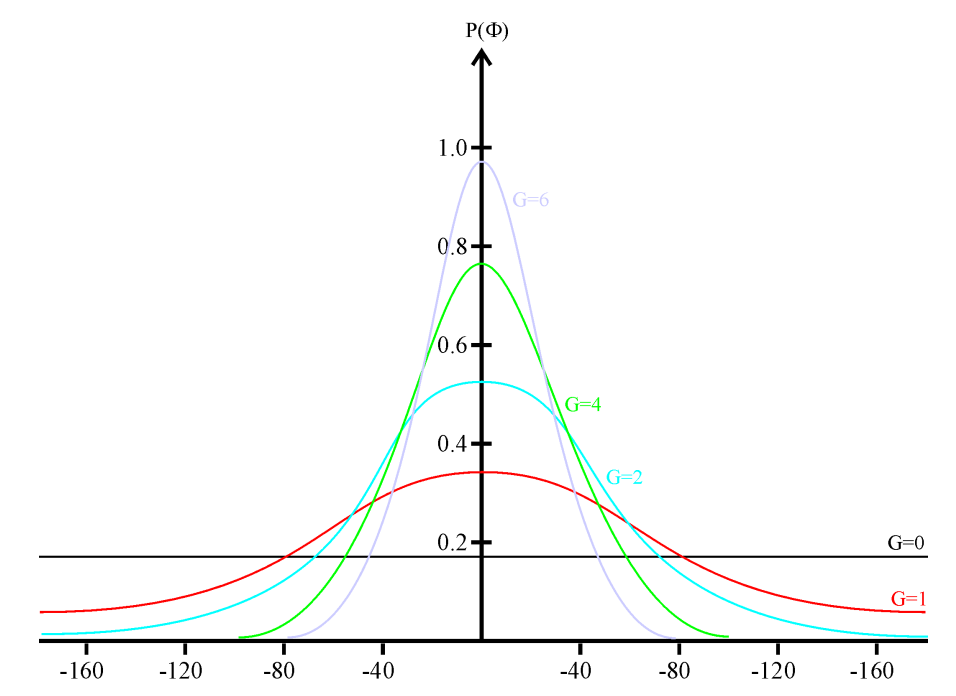

Figure 2.3

The Cochran-distribution for $\Phi_{\mathbf{h}, \mathbf{k}} . G$ is defined as $2 N^{-1 / 2}\left|\mathbf{E}_{\mathbf{h}} \mathbf{E}_{\mathbf{k}} \mathbf{E}_{-\mathbf{h}-\mathbf{k}}\right|$.

where $\varepsilon$ is the statistical weight of reflection $\mathbf{h}$, and the average is taken in resolution shells. It can be shown that the phase $\varphi_{\mathbf{h}}$ given the values of $\left|\mathbf{E}_{\mathbf{h}}\right|,\left|\mathbf{E}_{\mathbf{k}}\right|,\left|\mathbf{E}_{-\mathbf{h}-\mathbf{k}}\right|, \varphi_{\mathbf{k}}$ and $\varphi_{\text {-h-k }}$ follows a von Mises-type distribution (Figure 2.3) given as (Cochran, 1955)

$$
P\left(\varphi_{\mathbf{h}}\right)=\frac{1}{2 \pi \mathrm{I}_{0}\left(2 N^{-\frac{1}{2}}\left|\mathbf{E}_{\mathbf{h}} \mathbf{E}_{\mathbf{k}} \mathbf{E}_{-\mathbf{h}-\mathbf{k}}\right|\right)} \exp \left[2 N^{-\frac{1}{2}}\left|\mathbf{E}_{\mathbf{h}} \mathbf{E}_{\mathbf{k}} \mathbf{E}_{-\mathbf{h}-\mathbf{k}}\right| \cos \left(\varphi_{\mathbf{h}}+\varphi_{\mathbf{k}}+\varphi_{-\mathbf{h}-\mathbf{k}}\right)\right]
$$

The phase distribution of the triplet structure invariant $\left(\Phi_{\mathbf{h}, \mathbf{k}}=\varphi_{\mathbf{h}}+\varphi_{\mathbf{k}}+\varphi_{-\mathbf{h}-\mathbf{k}}\right)$ is centred on zero, and the greater the value of $N^{-1 / 2}\left|\mathbf{E}_{\mathbf{h}} \mathbf{E}_{\mathbf{k}} \mathbf{E}_{\text {-h-k }}\right|$, the more reliable is this phase value (Figure 2.4).

(a)

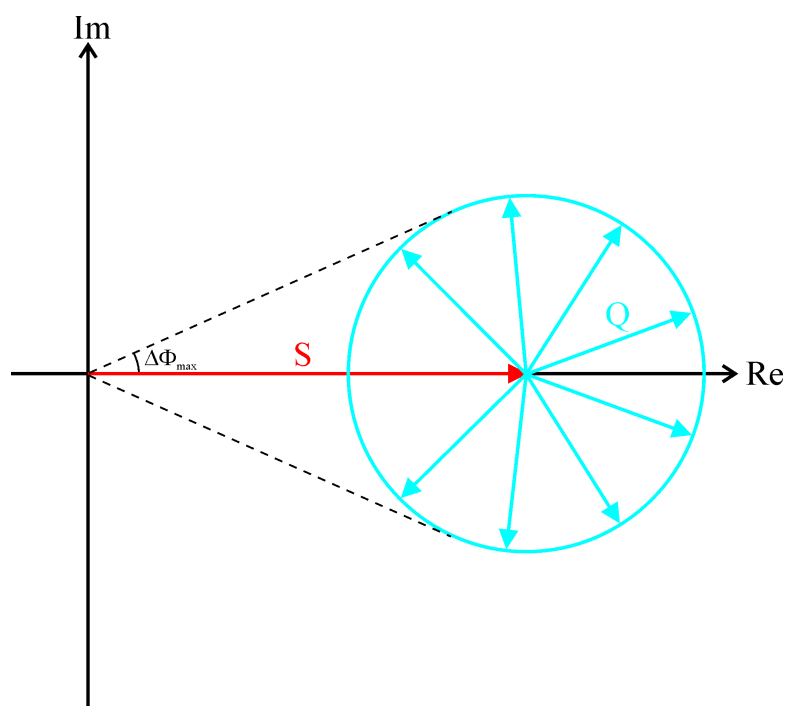

(b)

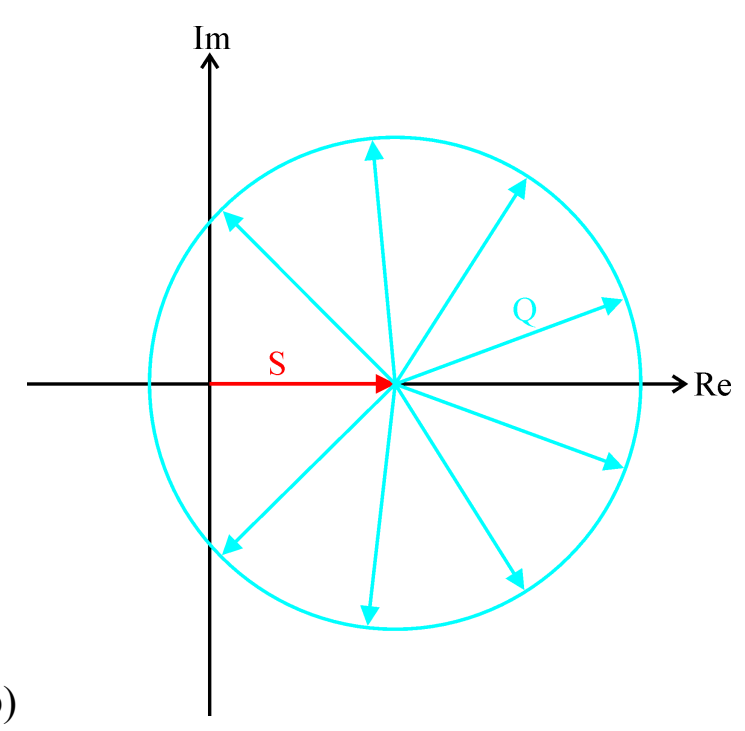

Figure 2.4

The strength of a triple-phase relation displayed graphically. (a) If $\mathbf{S}=k\left(\left|\mathbf{E}_{\mathbf{h}}\right|^{2}+\left|\mathbf{E}_{\mathbf{k}}\right|^{2}+\left|\mathbf{E}_{-\mathbf{h}-\mathbf{k}}\right|^{2}-2\right)$ is large ( $k$ is a constant, $\mathbf{S}$ is a real number), then the resulting phase can be estimated reliably. (b) If $\mathbf{S}$ is small, then the component $\mathbf{Q}$, whose value is unpredictable if no prior information about the interatomic vectors is available, dominates and no phase estimate is possible. $\mathbf{E}_{\mathbf{h}} \mathbf{E}_{\mathbf{k}} \mathbf{E}_{-\mathrm{h}-\mathrm{k}} \approx \mathbf{S}+\mathbf{Q}$. 
However, usually several triple-phase relations can be formed for reflection $\mathbf{h}$ and these can be combined to give a better estimate for $\varphi_{\mathbf{h}}$ (Karle \& Hauptman, 1956):

$$
\tan \varphi_{\mathbf{h}}=-\frac{\sum_{\mathbf{k}}\left|\mathbf{E}_{\mathbf{k}} \mathbf{E}_{-\mathbf{h}-\mathbf{k}}\right| \sin \left(\varphi_{\mathbf{k}}+\varphi_{-\mathbf{h}-\mathbf{k}}\right)}{\sum_{\mathbf{k}}\left|\mathbf{E}_{\mathbf{k}} \mathbf{E}_{-\mathbf{h}-\mathbf{k}}\right| \cos \left(\varphi_{\mathbf{k}}+\varphi_{-\mathbf{h}-\mathbf{k}}\right)}
$$

This equation (the tangent formula) is the basis of most conventional direct phasing procedures. The general strategy is to select reflections with large $\left|\mathbf{E}_{\mathbf{h}}\right|$ values, assign random phases to them and iteratively determine phases in turn for each of the selected reflections until convergence. A figure-of-merit is then calculated for each trial and the process is repeated several times (100-5000). Standard figures-of-merit comprise a combination of several factors like negative quartet phase relations and $R_{\alpha}$ (the agreement between the observed and calculated reliability parameter of triple-phase relations). The best phase set is then selected, and an electron density map is calculated (with only the phased strong reflections or after a phase extension to weaker reflections) that is peak-searched. This procedure can routinely solve small-molecule structures up to 100 unique atoms, but tends to lose enantiomorph discrimination and drifts towards a pseudo-centrosymmetric solution as the number of atoms increase. A relatively complete dataset to at least $1.2 \AA$ is a prerequisite.

\section{Dual-space recycling}

This modification of traditional direct phasing procedures can extend the limitation on the size of structures that can be solved by an order of magnitude, although the availability of high-resolution data is still a prerequisite (Sheldrick et al., 2001). This procedure starts from random atoms and calculates a phase set from them. This phase set can then be refined by the tangent formula or by the minimal function:

$$
R(\Phi)=\frac{\sum_{\mathbf{h}, \mathbf{k}} 2 N^{-\frac{1}{2}}\left|\mathbf{E}_{\mathbf{h}} \mathbf{E}_{\mathbf{k}} \mathbf{E}_{-\mathbf{h}-\mathbf{k}}\right|\left[\cos \Phi_{\mathbf{h}, \mathbf{k}}-\frac{\mathrm{I}_{1}\left(2 N^{-\frac{1}{2}}\left|\mathbf{E}_{\mathbf{h}} \mathbf{E}_{\mathbf{k}} \mathbf{E}_{-\mathbf{h}-\mathbf{k}}\right|\right)}{\mathrm{I}_{0}\left(2 N^{-\frac{1}{2}}\left|\mathbf{E}_{\mathbf{h}} \mathbf{E}_{\mathbf{k}} \mathbf{E}_{-\mathbf{h}-\mathbf{k}}\right|\right)}\right]^{2}}{\sum_{\mathbf{h}, \mathbf{k}} 2 N^{-\frac{1}{2}}\left|\mathbf{E}_{\mathbf{h}} \mathbf{E}_{\mathbf{k}} \mathbf{E}_{-\mathbf{h}-\mathbf{k}}\right|}
$$

The resulting phases are used to calculate an electron density map, which is then peaksearched to obtain new atom positions. This list of atoms can be manipulated and the new atoms are used to calculate a new phase set. This procedure is repeated several times, and at the end a figure-of-merit is calculated, which is either the value of the minimal function or the correlation coefficient between $\left|\mathbf{E}_{\text {obs }}\right|$ and $\left|\mathbf{E}_{\text {calc }}\right|$ (Fujinaga and Read, 1987). 
To increase efficiency, starting atoms can be selected so that they are consistent with the Patterson-function (Patterson seeding). In this procedure, a random peak is selected among strong Patterson-peaks; this potential two-atom vector is placed in the unit cell at random positions and Patterson deconvolution is performed with the most promising one. The resulting atom set is used as starting atoms. An increase in search efficiency can also be achieved by the application of the random omit technique in the real space cycle, in which about $30 \%$ of the peaks are discarded at random. If figures-of-merit indicate a potential solution, a few cycles of peaklist-optimisation can also be performed, which is a modification of the dual-space recycling that tests each atom found in the real-space cycle and eliminates it if an increase in the correlation coefficient can be observed. However, as this algorithm is computationally very expensive, this should only be performed for promising tries.

Dual-space recycling is a great advance over traditional direct methods, and the largest structure solved so far contains more than 2000 atoms. The availability of atomic resolution data is a requirement, although there are cases reported where lower resolution data proved to be sufficient for structure solution (Usón et al., 1999). Experience suggests that the presence of heavier atoms significantly increases the success rate and thereby extends limitations on the size of the structure, and probably also on the resolution limit.

\section{Molecular replacement}

This method can be regarded as the opposite of direct methods since it does not require high resolution data, but the availability of a model structure that is similar to the unknown structure. The whole procedure is based on the Patterson-function:

$$
\mathrm{P}(\mathbf{u})=\frac{1}{V} \sum_{\mathbf{h}}\left|\mathbf{F}_{\mathbf{h}}\right|^{2} \cos (2 \pi \mathbf{h u})
$$

which can be calculated directly from the measured data ( $V$ is the volume of the unit cell). It can be demonstrated that the Patterson-function is a vector map, i.e. peaks occur in the Patterson-map at positions that correspond to interatomic vectors. As the number of peaks in the Patterson-function is $n^{2}-n+1$ (where $n$ is the number of atoms in the unit cell), heavy overlap of peaks can be expected even for moderately sized structures.

Assume that a model structure homologous to the one in the asymmetric unit is available. In this case, the phase problem can be overcome by transferring the phases calculated from the model to the observed reflections. However, this would require accurately orienting and positioning the model in the unit cell, the content of which is unknown. 
Thus, before phases could be obtained, the orientation and the position of the model have to be established (Rossmann \& Arnold, 1993).

\section{The rotation search}

It is common practice to separate the inherently six-dimensional problem into two threedimensional ones. This can be achieved by realising that Patterson peaks arising from interatomic vectors among atoms of one molecule (self-Patterson vectors) do not depend on the position of the molecule but only on its orientation. If the Patterson-function could be divided into peaks from self-Patterson vectors and cross-Patterson vectors (vectors between atoms of neighbouring molecules), the observed self-Patterson map and the one calculated from the model in the same orientation would be identical. Although in general such a separation cannot be achieved for the observed Patterson-function, a good estimation can be given by assuming that self-Patterson vectors are short, while cross-Patterson vectors are long. The rotation function, which is the overlap between the observed and calculated Patterson-functions is defined as

$$
\mathrm{R}(\mathbf{C})=\int_{U} \mathrm{P}_{\text {obs }}(\mathbf{u}) \mathrm{P}_{\text {calc }}(\mathbf{C u}) d \mathbf{u}
$$

where $\mathbf{C}$ is a rotation matrix and the integration is performed for volume $U$ that contains predominantly self-Patterson vectors. This volume is usually chosen as a sphere with radius $r$ that is about $70-80 \%$ of the maximum distance from the centre of mass. For a globular protein molecule this radius would include approximately the same percentage of all self-Patterson vectors and only a small percentage of cross-Patterson vectors that only increase the noise. Thus, by selecting the integration sphere too large, the noise from cross-Patterson vectors increases and would at some point conceal the solution. On the other hand, too small a radius would decrease the discrimination of solutions from noise peaks, so an optimum in $r$ should be found. This requires a certain degree of experimenting with the parameter, especially for elongated molecules. However, if the Patterson-function is such that no separation of selfPatterson and cross-Patterson vectors can be achieved (e.g. tight packing), the procedure is likely to fail.

\section{The translation search}

When the orientation of the molecule has been established, the position can be deduced by moving the molecule on a suitable grid and calculating $R_{1}$ [equation (43)] or the correlation coefficient. The correlation coefficient is more reliable as it is independent of the scale factor 
between calculated and measured intensities. An alternative approach is the use of the translation function

$$
\mathrm{T}(\mathbf{m})=\int_{V} \mathrm{P}_{\text {obs }}(\mathbf{u}) \mathrm{P}_{\text {calc }}(\mathbf{u}, \mathbf{m}) d \mathbf{u}
$$

where $\mathbf{m}$ is the position vector of the centre-of-mass from the crystal origin and $V$ is the volume of the asymmetric unit. In the space group P1 no translation search is needed for the first molecule, as the crystal origin can be chosen arbitrarily.

\section{Six-dimensional search}

Although computationally demanding, a six-dimensional search can also be performed (Kissinger et al., 1999). This is likely to give better results when the above assumptions do not hold, i.e. for elongated molecules or when the solvent content is low, as it does not require the separation of self- and cross-Patterson peaks. Another condition under which this method may prove superior is the presence of non-crystallographic symmetry with high degree; in that case the rotation search is also likely to fail, since the observed Patterson-function is the overlap of several copies of the one calculated from the model and the maximum value of the rotation function is inversely correlated with the degree of non-crystallographic symmetry, and thus may not discriminate well from noise peaks.

\section{MODEL BUILDING}

After structure solution, approximate phases are available to calculate an electron density map. The distribution of electron density has to be interpreted and modelled by atoms or molecules that are arranged in a chemically sensible way. However, for an ab initio direct method structure solution, atomic positions are written out and they only have to be assigned to appropriate atoms. On the other hand, if the structure is solved with molecular replacement, the homologous structure used for solution can be taken as an initial model, and model building is reduced to correcting the differing parts.

Model building can be problematic if the starting phases are not good enough to obtain an interpretable electron density map (e.g. for isomorphous replacement and anomalous scattering techniques). In this case, density modification can be used. In this procedure, an electron density map is calculated from the observed structure factors and phases, and it is modified according to prior knowledge. The modified map is transformed back and new structure factor amplitudes and phases are obtained. The new phases are then combined with the old ones, while the amplitudes are discarded (although they may be used for calculating 
weights in the phase recombination stage). Prior knowledge of the electron density is the flatness of the solvent region (solvent flattening), the magnitude distribution of electron density in the protein region (histogram matching) or the identity of electron density map in certain regions of the unit cell (NCS-averaging). Even in the case of molecular replacement, solvent flattening can prove useful, since it can reduce model bias inherently present in the method.

\section{STRUCTURE REFINEMENT}

When an interpretation of the electron density in form of an atomic model is available, this model is usually fitted to the measured data by suitable refinement procedures. The number of parameters to be determined depends on the resolution: for high resolution it is common to fit nine parameters for an atom (coordinates + anisotropic vibrations), for medium resolution four parameters (coordinates + isotropic vibration), while for low resolution structures it is usual to group the temperature factors and to refine atomic coordinates only as torsion angles, i.e. less than four parameters for an atom. A general formula for the calculation of structure factors can be given as (Cruickshank, 1970)

$$
\mathbf{F}(\mathbf{S})=\sum_{i} f_{i}^{0}(|\mathbf{S}|) \mathrm{q}(\mathbf{S}) \exp \left(2 \pi i \mathbf{r}_{i} \mathbf{S}\right)
$$

where $f_{i}^{0}$ is the scattering factor of atom $i$ not subject to vibration, $\mathbf{r}_{i}$ is the position vector of atom $i$ and $\mathrm{q}(\mathbf{S})$ describes the effect of atomic vibrations. This latter is approximated by

$$
\mathrm{q}(\mathbf{S})=\exp \left(-2 \pi^{2} U_{i}|\mathbf{S}|^{2}\right)
$$

in the medium resolution case, where $U_{i}$ is the mean square amplitude of vibration in any direction, while in the high-resolution case

$$
\mathrm{q}(\mathbf{S})=\exp \left(-2 \pi^{2} \mathbf{S}^{+} \mathbf{U}_{i} \mathbf{S}\right)
$$

where $\mathbf{U}_{i}$ is a symmetric tensor and $\mathbf{S}^{+}$is the transpose of vector $\mathbf{S}$. The agreement of the model with the observed data is commonly described by the crystallographic $R$-factor

$$
R_{1}=\frac{\sum_{\mathbf{h}}|| \mathbf{F}_{o}(\mathbf{h})|-| \mathbf{F}_{c}(\mathbf{h}) \mid}{\sum_{\mathbf{h}}\left|\mathbf{F}_{o}(\mathbf{h})\right|}
$$

where the summation is performed usually for $\left|\mathbf{F}_{o}\right|$-values above a certain threshold. 


\section{Least-squares minimisation}

The best fit of the model to the observed intensities can be obtained by minimising the quantity

$$
M_{1}=\sum_{F>4 \sigma(F)} w_{\mathbf{h}}\left(\left|\mathbf{F}_{o}(\mathbf{h})\right|-\left|\mathbf{F}_{c}(\mathbf{h})\right|\right)^{2}
$$

or

$$
M_{2}=\sum_{\mathbf{h}} w_{\mathbf{h}}\left(\left|\mathbf{F}_{o}(\mathbf{h})\right|^{2}-\left|\mathbf{F}_{c}(\mathbf{h})\right|^{2}\right)^{2}
$$

Equation (44) is usually employed in the structure refinement of macromolecules, while for small molecular refinement equation (45) is dominant. Advantage of (44) is the smaller number of data used in the procedure; the cutoff has to be used since for weak or negative reflections the estimation of $\sigma\left(\left|\mathbf{F}_{\mathbf{h}}\right|\right)$ from the formula

$$
\sigma(|\mathbf{F}|)=\frac{\sigma\left(|\mathbf{F}|^{2}\right)}{2|\mathbf{F}|}
$$

is not valid any more, and the weights usually depend on the associated standard deviation [e.g. $\left.w_{\mathbf{h}}=1 / \sigma^{2}\left(\left|\mathbf{F}_{\mathbf{h}}\right|\right)\right]$. On the other hand, $\sigma\left(\left|\mathbf{F}_{\mathbf{h}}\right|^{2}\right)$ is an experimental quantity, and therefore no assumptions are involved in the derivation. Thus, using equation (45), all data can be used in the refinement, which is beneficial in pseudo-symmetric structures where low-intensity reflections prove to be important. In the latter case, weights are usually given in the form

$$
w=\left[\sigma^{2}\left(\left|\mathbf{F}_{o}\right|^{2}\right)+(g P)^{2}\right]
$$

where $P=1 / 3\left|\mathbf{F}_{o}\right|^{2}+2 / 3\left|\mathbf{F}_{c}\right|^{2}$, and the best value of $g$ can be found automatically. The solution proceeds by evaluating first and second derivatives and using a full-matrix inversion or by conjugate gradient minimisation. The full-matrix approach has the advantage that standard uncertainties are also obtained (equal to diagonal elements of the inverse normal matrix), but the required storage and the CPU-time required for matrix inversion scale with the second and third power of the number of parameters, respectively. The conjugate gradient optimisation scales with the first power of the number of parameters, and is numerically more stable than matrix inversion.

\section{Maximum likelihood refinement}

The least-squares technique can be regarded as a special case of maximum likelihood. However, the assumptions under which maximum likelihood reduces to least squares are only 
poorly justified in the case of refinement of atomic models for protein structures (Pannu \& Read, 1996). These assumptions can be summarised as follows:

(a) the deviation between $\left|\mathbf{F}_{o}\right|$ and $\left|\mathbf{F}_{c}\right|$ can be given as a Gaussian function.

(b) the mean deviation is zero.

(c) the standard deviation of the Gaussian is independent of the parameters of the atomic model.

The distribution of the true structure factor is best approximated by a Gaussian centred on $D \mathbf{F}_{c}($ Read, 1990), where

$$
D=\exp \left[-\pi^{3}\langle\Delta \mathbf{r}\rangle^{2}\left(\frac{\sin \Theta}{\lambda}\right)^{2}\right]
$$

and $\Delta \mathbf{r}$ is the coordinate error, i.e. the distribution is Gaussian in the complex plane. Therefore, the distribution $\left|\mathbf{F}_{o}\right|-\left|\mathbf{F}_{c}\right|$ will follow a Rice-distribution, and in the general case, especially in the initial stages, a maximum likelihood target is more appropriate in refinement. However, the procedure involves a huge increase in computational demand or many approximations to make it more efficient.

\section{Restrained refinement}

In macromolecular refinement the number of observations is comparable to that of parameters. However, this ratio can be improved by introducing a priori knowledge in form of restraints. A restraint is treated as an additional "observation" with a standard deviation and is included in the target function that gets minimised. Common restraints include:

(a) bond length restraints. Distortion of bond lengths requires considerable energy and the resolution is often not good enough to resolve atoms, so these can be restrained to have values determined using high-resolution small-molecular structures with small standard deviation.

(b) bond angle restraints. In this case the energy demand of distortion is significantly less, but it may still be justified to restrain these values, but with a higher standard deviation.

(c) planarity restraints. These are usually employed for chemical moieties that are believed to be planar under normal circumstances. 
(d) anti-bumping. The assumption that two non-bonded atom should not be nearer than the sum of their van der Waals distances is usually valid. This restraint is special from the point that in one direction the deviation should not be penalised.

(e) anisotropic displacement parameter restraints. Anisotropic temperature factor refinement increases the number of parameters considerably, so the inclusion of these restraints is often necessary.

Even when high-resolution data are available, for a protein structure the use of restraints is recommended.

\section{Common problems}

In protein crystallography the main problem is usually constituted by the low data-toparameter ratio in structure refinement, and the tendency to overfit the data by using too many parameters, which in compensation for other factors assume physically unrealistic values. To determine whether the addition of a set of parameters results in a better description of electron density, a given percentage of the reflections is usually excluded from the refinement and used for the calculation of $R_{\text {free }}$ (Brünger, 1992). This is defined in an analogous way to $R_{1}$ [equation (43)], but the summation goes for reflections not used in the refinement. $R_{\text {free }}$ reflections are selected randomly or in thin shells when non-crystallographic symmetry is present. Although this is a good global guide for describing the course of the refinement, it is not sensitive to small changes, e.g. modelling discrete disorder for a sidechain.

Another important issue is the description of disordered solvent always present in protein crystals (Moews \& Kretsinger, 1975). There is indication that Babinet's principle that is widely used in solvent modelling is not satisfactory and especially for crystals with high solvent content, the value of standard agreement indicators is often too high. 


\section{CRystal StRuctures OF CEPHAibols}

\section{INTRODUCTION}

Cephaibols comprise a group of peptaibol antibiotics and have been isolated from the soil fungus Acremonium tubakii, DSM 127741. Apart from their considerable antibacterial potency, it has been established that cephaibols possess pronounced anthelmintic action and activity against ectoparasites (Schiell et al., 2001). Other surprising biological properties, like induction of pigment formation in Phoma destructiva and potential neuroleptic effects have also been reported (Ritzau et al., 1997; Schlegel et al., 2002).

The amino acid sequence of cephaibols shows marginal variation among group members (Figure 3.1), the main difference being some methyl groups at positions 5, 8 and 12 . However, as there is at least a 10 -fold variation in the antibacterial potency, the presence or absence of these methyl groups seems to have a substantial effect upon antimicrobial action. Unlike other types of antibiotics, peptaibols frequently occur naturally as microheterogeneous mixtures; this peculiarity has long been established and attributed to their non-ribosomal

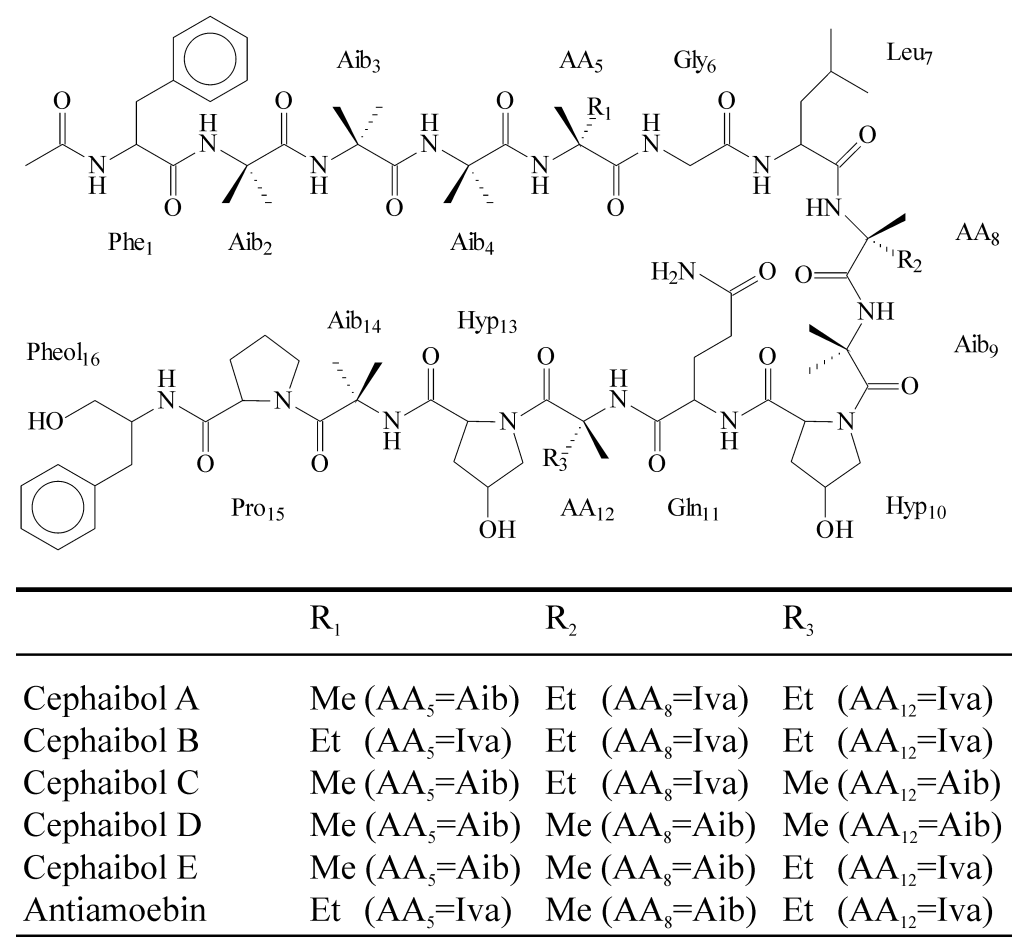

Figure 3.1

Amino acid sequence of members of the cephaibol series and comparison with antiamoebin. 
peptide synthesis, in which Aib is frequently replaced by other $\alpha, \alpha$-dialkylated amino acids (Leclerc et al., 1998).

Three-dimensional structures of various peptaibols have been determined by X-ray diffraction for alamethicin (Fox \& Richards, 1982), [Leu1]zervamicin (Karle et al., 1991), antiamoebin (Karle et al., 1998; Snook et al., 1998), trichotoxin (Chugh et al., 2002) and ampullosporin (Kronen et al., 2003). The NMR solution structures of chrysospermin C (Anders et al., 2000), antiamoebin (Galbraith et al., 2003) and zervamicin IIb (Balashova et al., 2000; Shenkarev et al., 2002) have also been reported. These molecules seem to adopt a common structural motif that consists of a long helical section that has a variable bend at a proline or a hydroxyproline residue near to the middle of the helix. Since the action of peptaibols is believed to derive from their interaction with biological phospholipid membranes, in which the formation of ion channels results in increased ion permeability (Menestrina et al., 1986), the length should be an important factor in determining the potency. Alamethicin with its 20 residues and moderate bending angle (about $30^{\circ}$ ) is more than $33 \AA$ long and therefore able to span biological membranes. Conductivity measurements with several artificial and naturally occurring membrane systems did show the formation of highly voltage sensitive and weakly cation selective membrane channels (Gordon \& Haydon, 1972; Hanke \& Boheim, 1980; Latorre \& Alvarez, 1981). The 18-residue trichotoxin and the 19-residue chrysospermin C possess similar structures, although they are shorter and their bending angles are markedly different $\left(10^{\circ}\right.$ and $38^{\circ}$, respectively). [Leu1]zervamicin consists of only 16 residues and its bending angle varies among the existing structures in the range $30-45^{\circ}$. Consequently, it is significantly shorter than alamethicin, only $29 \AA$ long, but conductivity measurements still prove the formation of membrane channels (Balaram et al., 1992). Moreover, it was found that in all crystal forms [Leu1]zervamicin molecules aggregated in a similar fashion to form water channels and suggested a gating mechanism for cation transport. The structure of antiamoebin has been determined independently in methanol (Snook et al., 1998) and in a partially membrane-mimetic environment (Karle et al., 1998). Although different crystal forms were obtained, the peptide conformation and even the molecular packing show strong similarity between the two structures. The 16-residue peptide, in comparison with [Leul]zervamicin, possesses a much sharper bend nearly $55^{\circ}$, but only marginally shorter than [Leu1]zervamicin. Initial membrane conductivity measurements failed to give evidence of membrane channel formation, and although later largely voltageinsensitive channel formation was observed in specific membrane media (Duclohier et al., 
1998), a hypothesis for an ion carrier mechanism was put forward. Ampullosporin consists of only 15 residues and a bending angle of about $60^{\circ}$ and therefore with a length of $20 \AA$ it is the shortest of all peptaibols with known three-dimensional structure.

Cephaibols, although similar to antiamoebin, were available as pure compounds that have marginal structural difference but wide variation in antibacterial potency and provided an opportunity to investigate the effect of small structural changes on backbone conformation and association properties.

\section{STRUCTURE DETERMINATION}

\section{Crystallisation}

Quantities of cephaibols were ranging from $200 \mathrm{mg}$ (cephaibol A) to $5 \mathrm{mg}$ (cephaibol D and E). Although crystallisation procedures for several peptaibol antibiotics had been available (Fox \& Richards, 1982; Karle et al., 1991; Karle et al., 1998; Snook et al., 1998), these were not feasible because of either the high amount of antibiotic required (e.g. antibiotic concentration up to $200 \mathrm{mg} / \mathrm{ml}$ ) or the danger of sample decomposition in a step (e.g. boiling). To render the crystallisation of all cephaibol samples possible, the vapour diffusion method was selected as the only one compatible with the limited availability of certain members and the expected high proportion of organic solvents. Initially, the published conditions suitably modified to hanging drop technique were tested, but no diffraction quality crystals were obtained. As the next step, screening was performed with cephaibol A, which was available in the largest amount. It was expected that a well-established crystallisation condition might also function for all of the different samples and demand only certain degree of optimisation, and screening was therefore conducted with adequate caution. Due to the low solubility of the peptide in water, dedicated screens with high organic solvent content available from Jena Bioscience were employed. Crystals were obtained from several conditions, but they had the same morphology, and for that reason, the condition yielding nearly diffraction quality crystals was selected and optimised. Eventually, high-quality crystals diffracting to atomic resolution were obtained by mixing $4 \mu \mathrm{l}$ of $5 \mathrm{mg} / \mathrm{ml}$ cephaibol A in ethanol:water=1:1 with $2 \mu \mathrm{l}$ of $0.1 \mathrm{M} \mathrm{NaAc} / \mathrm{HAc} \mathrm{pH}=4.6$ and $35 \%$ ethanol. Cephaibol B crystals were obtained from slightly different conditions (33\% ethanol vs. $35 \%$ ethanol). Cephaibol C required comparatively extensive alteration and was grown by mixing $2 \mu \mathrm{lof} 40 \mathrm{mg} / \mathrm{ml}$ cephaibol C in ethanol:water=1:1 with $2 \mu \mathrm{l}$ of $0.1 \mathrm{M} \mathrm{NaAc} / \mathrm{HAc} \mathrm{pH}=4.6$ and $37 \%$ ethanol. Initial crystals 
from cephaibol E were obtained using a procedure similar to cephaibol C. Cephaibol D gave spherulites from these conditions, but microcrystals could be obtained by mixing equal volumes of ethyleneglycol:ethanol $=55: 45$ with $20 \mathrm{mg} / \mathrm{ml}$ cephaibol D in ethanol:water=1:1. Crystal growth took from several days (cephaibol A, B and C) to several months (cephaibol D and E). Crystal dimensions varied from $0.7 \cdot 0.4 \cdot 0.1 \mathrm{~mm}^{3}$ (for cephaibol A, B and C) down to $0.1 \cdot 0.05 \cdot 0.01 \mathrm{~mm}^{3}$ (cephaibol D and E).

\section{Measurement}

Before measurement, a suitable crystal was selected and soaked in a cryoprotectant solution consisting of the reservoir solution and $20 \%$ ethyleneglycol, mounted with an adequately sized loop and shock-frozen in a cold nitrogen stream. Diffraction images were collected on a Bruker rotating anode with Osmic focusing mirrors and a SMART6000 4K CCD detector. Built-in functionalities of the program Proteum were used for indexing and cell refinement; integration was performed with SAINT and scaling with SADABS. XPREP was employed for space group determination and for the calculation of statistics (Bruker Nonius, 2002). Unit cell parameters and intensity statistics are shown in Table 3.1.

\begin{tabular}{|c|c|c|c|c|c|}
\hline & Cephaibol A & Cephaibol B & Cephaibol C & Cephaibol D & Cephaibol E \\
\hline Space group & $\mathrm{P} 2{ }_{1}{ }_{1} 2$ & $\mathrm{P} 2{ }_{1}$ & $\mathrm{P} 2{ }_{1} 2_{1} 2_{1}$ & & \\
\hline Unit cell parameters & $\begin{array}{l}\mathrm{a}=30.534 \AA \\
\mathrm{b}=37.787 \AA \\
\mathrm{c}=9.115 \AA\end{array}$ & $\begin{array}{l}a=32.146 \AA \\
b=9.126 \AA \\
c=37.982 \AA \\
\beta=111.36^{\circ}\end{array}$ & $\begin{array}{l}\mathrm{a}=9.002 \AA \\
\mathrm{b}=28.619 \AA \\
\mathrm{c}=41.100 \AA\end{array}$ & $\begin{array}{l}a=9.275 \AA \\
b=29.590 \AA \\
c=39.724 \AA \\
\alpha=68.80^{\circ} \\
\beta=83.30^{\circ} \\
\gamma=80.98^{\circ}\end{array}$ & $\begin{array}{l}a=9.250 \AA \\
b=29.250 \AA \\
c=39.381 \AA \\
\alpha=69.03^{\circ} \\
\beta=85.45^{\circ} \\
\gamma=81.65^{\circ}\end{array}$ \\
\hline No. of reflections & 67303 & 73436 & 76788 & & \\
\hline $\begin{array}{l}\text { No. of unique } \\
\text { reflections }\end{array}$ & 7025 & 16045 & 8776 & & \\
\hline Resolution $(\AA)$ & $0.95(1.05-0.95)$ & $0.89(1.00-0.89)$ & $0.89(1.00-0.89)$ & & \\
\hline Completeness & $98.6 \%(96.6 \%)$ & $97.0 \%(90.0 \%)$ & $99.3 \%(97.6 \%)$ & & \\
\hline Redundancy & $9.45(4.66)$ & $4.44(2.16)$ & $8.69(4.36)$ & & \\
\hline$<I / \sigma(I)>$ & $29.21(10.67)$ & $23.29(10.37)$ & $50.44(26.51)$ & & \\
\hline$R_{\mathrm{int}}$ & $0.0431(0.1156)$ & $0.0437(0.0786)$ & $0.0306(0.0349)$ & & \\
\hline
\end{tabular}

Table 3.1

Data collection statistics. For cephaibol D and E the primitive unit cells are shown. For cephaibol D, this cell can be converted to a $\mathrm{C}$-centred monoclinic cell with cell dimensions $\mathrm{a}=58.448 \AA$, $\mathrm{b}=9.275 \AA, \mathrm{c}=40.041 \AA, \beta=112.64^{\circ}$. Values in parentheses refer to the last resolution shell. $R_{\text {int }}=\Sigma|I-<I>| / \Sigma I$. 

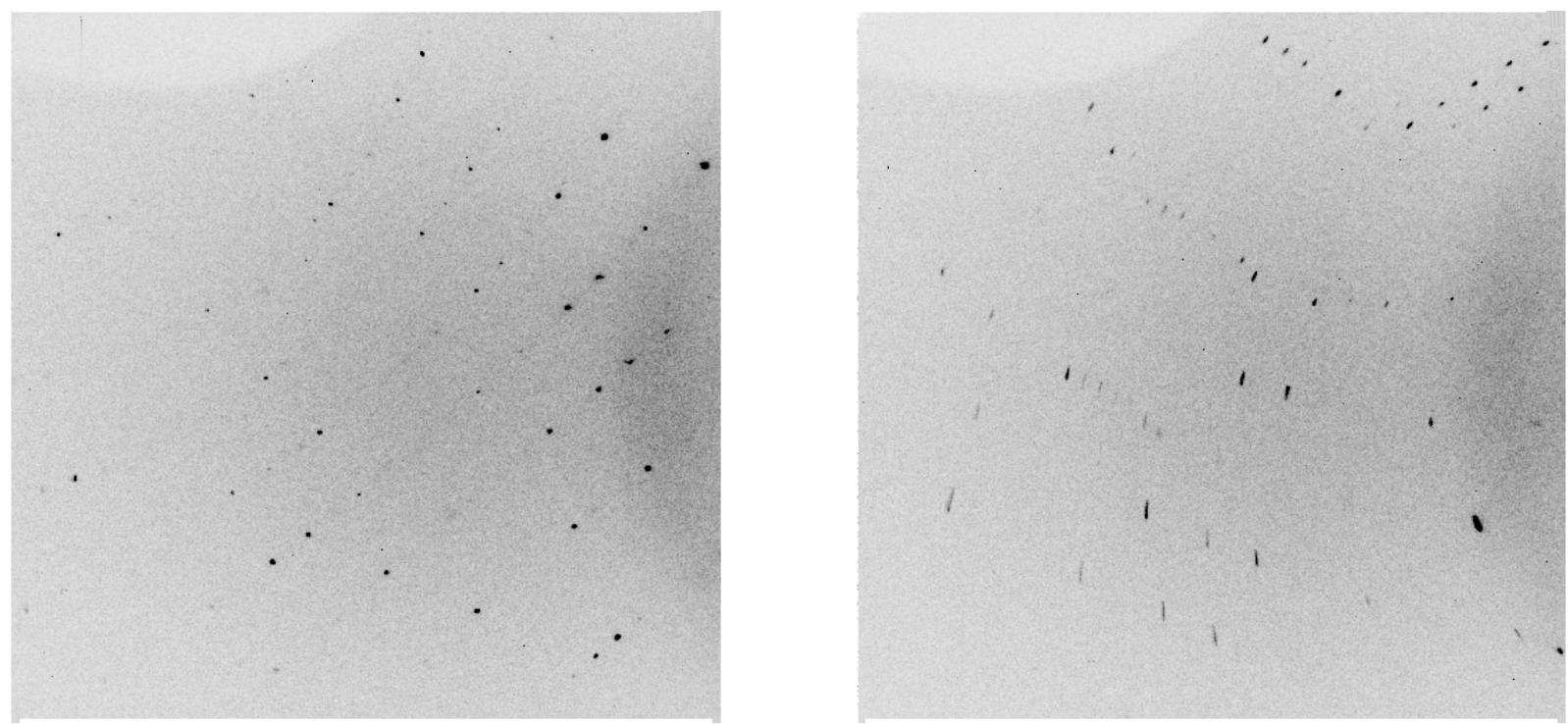

Figure 3.2

Diffraction images of cephaibol B showing high anisotropic mosaicity. However, as both the $R_{\text {int }}$ and the refinement $R$-factors are similar to those of cephaibol $\mathrm{A}$ and $\mathrm{C}$, it can be regarded that data processing software can handle such property when data are collected in an appropriate way. These images were taken at $2 \Theta=80^{\circ}$ and with an $\omega$-difference of approximately $90^{\circ}$. The shadow in the upper left corner is caused by the nozzle of the cryostream.

It was astonishing to realise that despite the high degree of structural homology and nearly identical crystallisation conditions, the crystals were not isomorphous and differed in unit cell parameters as well as in space group. Crystallising homologous samples in a similar way therefore does not necessarily yield the same crystal form, and crystal packing may be extremely sensitive to small structural differences even if they are not involved in crystal contacts. This observation also implies that as tiny difference as a single atom may prevent a sample from crystallisation, but on the other hand, puts forward the theory that homologous substances are likely to crystallise under similar conditions and justifies the development of crystallisation screens specialised on protein classes.

Full datasets were collected for cephaibol A, B and C and processed without any major difficulty. The crystals gave strong diffraction and the only resolution-limiting condition proved to be the maximum detector $2 \Theta$-angle. Cephaibol B exhibited significant anisotropy of mosaicity, but this property was routinely handled by SAINT (Figure 3.2) and its effect is present neither in data quality nor in structure refinement. Frames for indexing were collected for cephaibol E, but as the diffraction limit was only $1.8 \AA$ and the crystal was nonmerohedrally twinned, no dataset was collected. It is worth noting that cephaibol E had a 
markedly different cell from cephaibol $\mathrm{C}$ in spite of the fact that cephaibol $\mathrm{E}$ could integrate into the crystal lattice of cephaibol $\mathrm{C}$ and was therefore expected to yield the same crystal form. Cephaibol D yielded crystals comparable in size and diffraction limit to those of cephaibol E, although the crystal characterised was proved to be single. It is noteworthy that the primitive cells of cephaibol D and E are very similar. The metric symmetry for cephaibol $\mathrm{D}$ was determined as C-centred monoclinic, but it is not known whether the Laue-symmetry corresponds to that or not. Cell dimensions of cephaibol $\mathrm{E}$ also allow conversion to a C-centred monoclinic cell, but the distortion in angles is significant; however, this may be a consequence of the reduced precision in cell determination due to non-merohedral twinning.

\section{Structure solution}

The asymmetric unit of cephaibols was sufficiently small and the resolution was sufficiently high to obtain structure solution without complication. SHELXD (Sheldrick et al., 2001) was employed to find the peptide atoms (FIND 120 for cephaibol A and C, which have one molecule in the asymmetric unit and FIND 240 for cephaibol B, where there are two molecules in the asymmetric unit), and the solvent molecules (three cycles of peaklist optimisation by allowing 10-15\% more atoms than expected from the structural formula). Cephaibol A had the highest success rate ( 9 out of 300 tries), followed by cephaibol B ( 5 out of 500) and then cephaibol C (3 out of 400). One try took about $70 \mathrm{~s}$ for cephaibol A and B and about $25 \mathrm{~s}$ for cephaibol $\mathrm{C}$ on a Pentium III $600 \mathrm{MHz}$ processor. The solution rate was therefore roughly equal, although the asymmetric unit of cephaibol B is nearly twice as large.

\section{Model building and refinement}

After successful solution, several cycles of temperature factor refinement were performed using the program SHELXL (Sheldrick \& Schneider, 1997) and peaks in the electron density have been assigned to corresponding atoms with the program XP. When virtually all peptide atoms had been found, the refinement was carried on with SHELXL using suitable bond length, bond angle, chiral volume and planarity restraints. Throughout the refinement, $2 m F_{o}-D F_{c}$ and $F_{o}-F_{c}$ type maps were displayed with the program XtalView (McRee, 1999), which was also used for manual editing of the structure and identifying disorder components. Water molecules were added with the program SHELXWAT and by hand. All non-hydrogen atoms were refined anisotropically with rigid bond, similarity, and 
for solvent molecules, approximately isotropic restraints; hydrogen atoms were included in later stages of refinement. Refinement details are shown in Table 3.2.

\begin{tabular}{llll}
\hline & Cephaibol A & Cephaibol B & Cephaibol C \\
\hline Resolution range $(\AA)$ & $37.79-0.95$ & $35.37-0.89$ & $28.62-0.89$ \\
$R_{1}[\mathrm{~F}>4 \sigma(\mathrm{F}) /$ all data $]$ & $0.0835 / 0.0872$ & $0.0709 / 0.0746$ & $0.0712 / 0.0719$ \\
$R_{\text {free }}[\mathrm{F}>4 \sigma(\mathrm{F}) /$ all data $]$ & $0.1082 / 0.1120$ & $0.0756 / 0.0793$ & $0.0758 / 0.0770$ \\
No. of parameters & 1429 & 3121 & 1294 \\
No. of restraints & 2016 & 4633 & 1519 \\
No. of peptide atoms & 119 & 240 & 118 \\
No. of non-hydrogen atoms & 133 & 268 & 135 \\
Solvent content & $21.9 \%$ & $20.0 \%$ & $23.0 \%$ \\
R.m.s.d. from ideal geometry & & & \\
\multicolumn{1}{c}{ Bond length $(\AA)$} & 0.017 & 0.021 & 0.018 \\
$\quad$ Angle distances $(\AA)$ & 0.035 & 0.040 & 0.028 \\
$\quad$ Common planes $(\AA)$ & 0.416 & 0.505 & 0.499 \\
\hline
\end{tabular}

Table 3.2

Model refinement statistics.

\section{STRUCTURE DESCRIPTION}

\section{Amino acid sequence}

The overall structures of the molecules were found to be very similar. All three cephaibols assume a helical conformation that is sharply bent at Hyp10. The bending angle is nearly $55^{\circ}$ and seems not to vary much. Before the structure determination it was unclear whether the peptides contained D- or L-isovaline, as the chemical structures had been established with NMR-spectroscopy and mass spectrometry. The maps unambiguously showed that all isovalines are present as D-isovaline (assuming that the common amino acids are present as L-enantiomers). 


\section{Backbone conformation}

\section{Cephaibol A}

Cephaibol A crystallises in the space group P $2{ }_{1} 2{ }_{1} 2$ with one molecule in the asymmetric unit. The whole molecule is well defined apart from two residues (Phl16 and Pro15) that each exhibit two distinct conformations. This 1:1 disorder implies the flipping of the proline ring into the other envelope conformation and the consequent motion of Phe16. Apart from this fact, the structure shows considerable homology to the previously determined peptaibol structures in terms of backbone conformation, secondary structure and intramolecular hydrogen bonding pattern. Accordingly, in spite of the high Aib content, residues 1 to 9 form an $\alpha$-helix, residues 10 -12 a short 3 -helix, while the conformation of residues 12-16 should be considered as two overlapping $\beta$-turns. The bend of the helix occurs at Hyp10, which is identical to that in antiamoebin and [Leu1]zervamicin, and the bending angle is comparable to that of antiamoebin.

\section{Cephaibol B}

Cephaibol B crystallises in the monoclinic space group $\mathrm{P} 2{ }_{1}$, but with very similar cell parameters to cephaibol A and therefore has two molecules in the asymmetric unit. Although the overall structures of the two molecules are similar, different regions were found to be discretely disordered. In the first molecule (molecule I), Hyp10, where the molecule is bent, shows a 9:1 disorder for the $\mathrm{OH}$ group being in axial and equatorial position, respectively. The backbone of molecule I from Iva12 to Phl16 inclusive also adopts two different conformations. This disorder may arise from the interaction with the second molecule (molecule II), in which the interacting region (from Ac0 to Aib3) is also disordered, allowing different hydrogen bonds between the two conformations. It is also noteworthy that in molecule II the $\mathrm{OH}$ group of Phl16 adopts two conformations, but the sum of occupancies turns out to be slightly higher than unity. This fact seems to be accounted for by mass spectrometric evidence that together with cephaibol B there is another compound present that has a sequence identical to that of the principal constituent, but with an aldehyde function instead of the alcohol group at Phl16 (Schiell et al., 2001). As a geminal diol, an aldehyde group would convert into two $\mathrm{OH}$ groups, and increase the occupancy for both disorder components. It should be emphasised that according to the electron density map, only a minority of the molecules ends in phenylalaninal and the rest have phenylalaninol with a disordered $\mathrm{OH}$ as $\mathrm{C}$-terminus. However, the presence of the aldehyde seems to be significant. 


\section{Cephaibol C}

Cephaibol $\mathrm{C}$ crystallises in the orthorhombic system with cell edges very similar to those of cephaibol A, but in the space group $\mathrm{P} 2{ }_{1} 2_{1} 2_{1}$. This difference in symmetry involves different interactions amongst the molecules and consequently different packing. The structure and packing of cephaibol $\mathrm{C}$ was found to be very similar to that of [Leu1]zervamicin, which crystallises in the space group $\mathrm{P} 2_{1}$, and contains no disorder. The electron density map showed the presence of some cephaibol E contamination, which manifested itself as Aib12 being partially Iva. This contamination originates from the retention times of cephaibol $\mathrm{C}$ and $\mathrm{E}$ being nearly identical, making these two components very difficult to separate (Schiell et al., 2001).

\section{Comparison of the structures}

To search for rigid fragments, the program ESCET (Schneider, 2002) was employed with coordinate estimated standard deviations generated from temperature factors using Cruickshank's DPI equation (Cruickshank, 1999) plus linear B-factor scaling. In total four different molecules were compared simultaneously and in a pairwise manner (side chain atoms were not included). On average, the molecules are about $20 \%$ identical within the experimental error (this number is a slight underestimation as the precision of the structures is relatively high), but in special cases the similarity reaches higher levels. Cephaibol A and C were found to share about $60 \%$ of identical structure, while cephaibol A also has about $60 \%$ in common with one of the cephaibol B molecules. Interestingly, the two cephaibol B molecules were found to be less similar, showing only $30 \%$ identity.

To identify any apparent motion, rigid atoms found by ESCET were fitted together with the program LSQKAB (Kabsch, 1976), and the whole molecules were transformed using the matrix resulting from this superposition. The molecules were then compared using computer graphics. In the case of cephaibol A and cephaibol C, the two structures fit very well onto each other from their N-terminus till position 12, where the sequence differs (Figure 3.3a). For cephaibol A and cephaibol B, the centres assume nearly identical conformation, only the termini seem to be flexible, and the sequence difference at position 5 seems not to be responsible for the conformational change (Figure 3.3b). Simultaneous superposition of all models reveals that despite the low structural identity nearly the whole N-terminal helix is rigid, while the C-terminus exhibits conformational diversity (Figure 3.3c). This finding can be confirmed by the analysis of difference-distance matrices that give no indication of a rigid, 


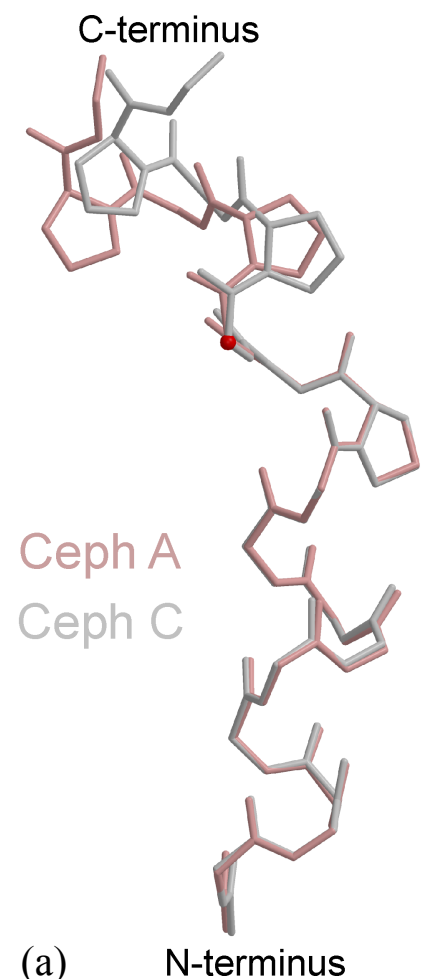

Figure 3.3

Conformation differences among cephaibol A, B and C. (a) More than half of cephaibol A is virtually identical with cephaibol $\mathrm{C}$ and the deviation starts at position 12 (marked with the red ball), where the sequence differs. (b) Cephaibol B fits very well on cephaibol A and position 5 (the difference in sequence) seems not to be responsible for the different conformations. (c) Simultaneous superposition of cephaibols reveals a rigid N-terminal helix, which includes two of three positions where the sequence may differ.

but smaller sized C-terminal helix and a flexible hinge region. The observed extensive disorder of C-termini also suggests that this region can assume multiple conformations.

\section{Crystal packing}

In the case of cephaibol $\mathrm{C}$, nearly the same arrangement was found as for antiamoebin and [Leu1]zervamicin, i.e. two $\mathrm{V}$-shaped molecules that are related by a translation along the crystallographic a axis face a third one and form a channel that looks like an X when viewed from the side (Figure 3.4). Strong intermolecular hydrogen bonds connect the three molecules, especially between $\mathrm{O}(\mathrm{Gly} 6)-\mathrm{N}^{\varepsilon}(\mathrm{Gln} 11)^{\prime}, \mathrm{O}^{\delta}(\operatorname{Hyp} 10)-\mathrm{N}^{\varepsilon}(\mathrm{Gln} 11)^{\prime}, \mathrm{O}^{\delta}(\operatorname{Hyp} 10)-$ $\mathrm{O}\left(\right.$ Leu7)', O(Leu7)-O $\mathrm{O}^{\delta}(\operatorname{Hyp} 10) ", \mathrm{~N}^{\varepsilon}(\mathrm{Gln} 11)-\mathrm{O}^{\delta}(\operatorname{Hyp} 10) "$ and $\mathrm{N}^{\varepsilon}(\mathrm{Gln} 11)-\mathrm{O}(\mathrm{Gly} 6) "$ (the apostrophes ' and " denote different symmetry equivalent molecules). This arrangement is believed to be representative of the functional membrane channel (Karle et al., 1991). 


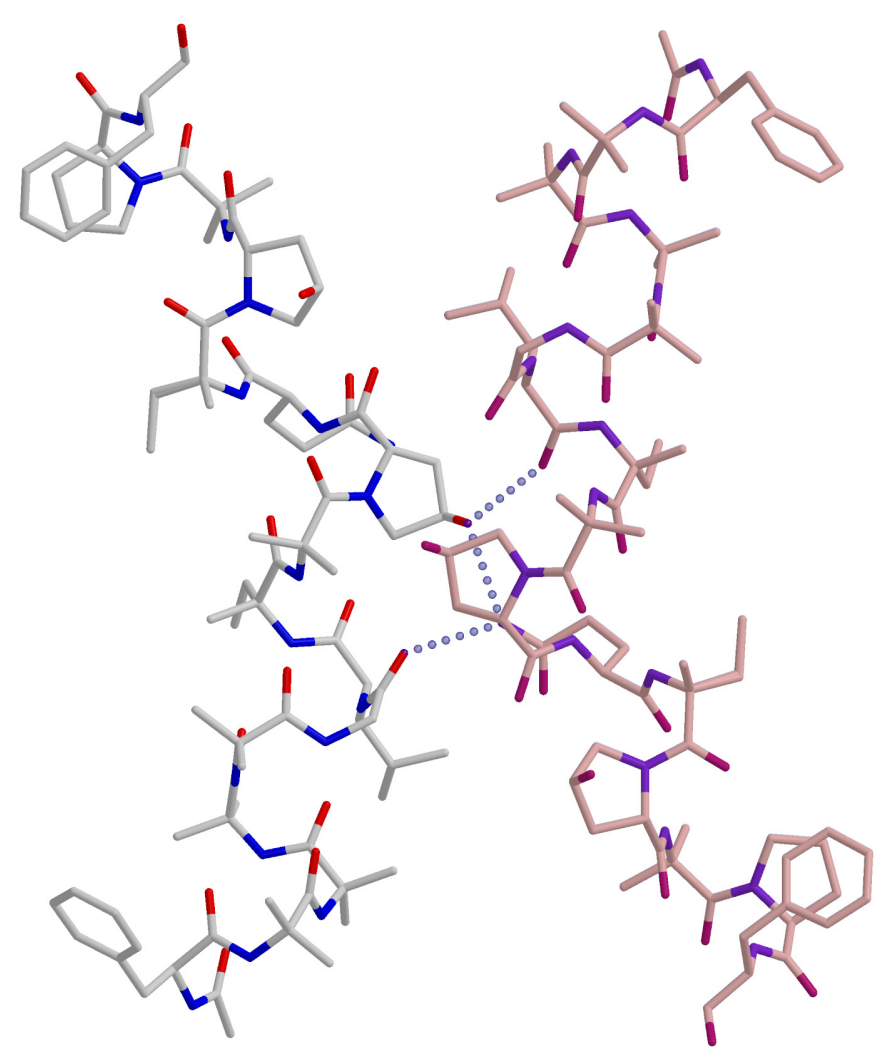

Figure 3.4

Three cephaibol $\mathrm{C}$ molecules adopt a channel-like arrangement (the left molecule represents the asymmetric unit, the right molecule is a symmetry equivalent that is designated by one apostrophe in the text and the third molecule, which is directly behind the right one, is designated by two apostrophes; hydrogen bonds that involve the third molecule are not shown).

Hydrogen bonds between N(Phe1)-O(Pro15)"', N(Aib2)-OH(Pheol16)'" and $\mathrm{O}^{\delta}($ Hyp13)$\mathrm{O}^{\varepsilon}(\mathrm{Gln} 11)^{\prime \prime \prime}$ connect to other symmetry related molecules and seem to be important in stabilising the crystal lattice.

Surprisingly, cephaibol A and cephaibol B adopt different packing motifs to cephaibol C. The main hydrogen bonds connect two termini and the centre of one molecule to the terminus of another. The molecules form a layer-like zigzag structure (Figure 3.5). There is no sign of any channel formation similar to that observed in the case of cephaibol C, and Hyp10, which was involved in most of the intermolecular interactions, is now pointing towards a water-filled cavity. In the case of cephaibol A the following hydrogen bonds are observed between the molecules: $\mathrm{N}(\mathrm{Phe} 1)-\mathrm{OH}(\mathrm{Pheol16A})$ ' is the head-to-tail interaction, where A refers to one of two distinct conformations with higher occupancy, while the 


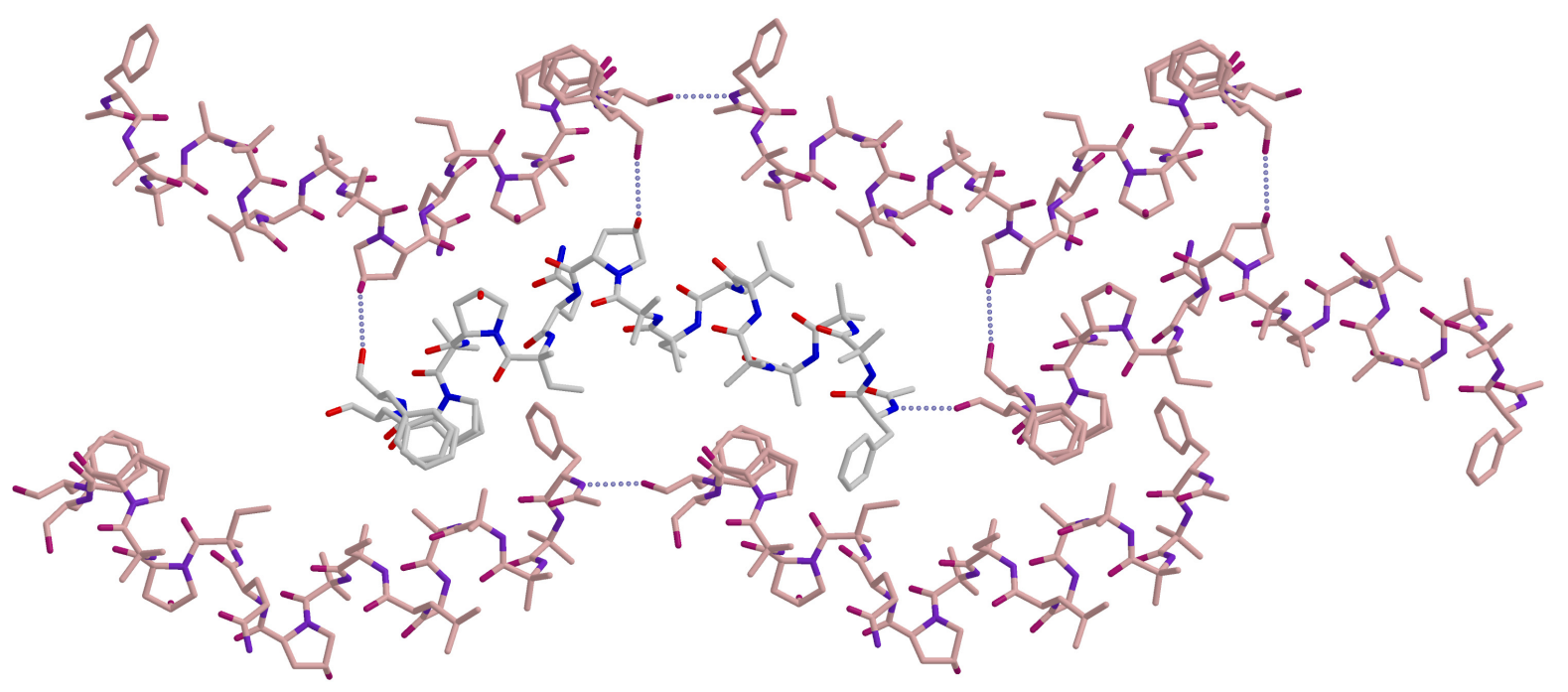

Figure 3.5

Cephaibol A and B (cephaibol A is shown) form a zigzag structure that is not indicative of membrane channel formation. Hydrogen bonds involving Gln11 are omitted, because they would involve molecules that are behind the layer that is shown.

$\left.\mathrm{O}^{\delta}(\mathrm{Hyp} 10)-\mathrm{OH}(\mathrm{Pheol16B}) ", \quad \mathrm{~N}^{\varepsilon}(\mathrm{Gln} 11)-\mathrm{O}^{\delta}(\mathrm{Hyp} 13)\right)^{\prime \prime}$ and $\mathrm{O}^{\varepsilon}(\mathrm{Gln} 11)-\mathrm{O}^{\delta}(\mathrm{Hyp} 13) " '$ interactions connect the centre of the molecule with the $\mathrm{C}$-termini of three molecules. This pattern also accounts for the disorder observed at the C-terminus, since $\mathrm{OH}(\mathrm{Pheol16})$ is able to participate in hydrogen bonds in both conformations, although the first is marginally more favourable. The two molecules in the cephaibol B crystal, although having the same packing arrangement as cephaibol A, interact at the termini by means of more hydrogen bonds: N(Phe1/I)-O(Pro15/II)'， N(Phe1/I)-OH(Pheol16B/II)', N(Aib2/I)-OH(Pheol16B/II)' and $\mathrm{N}($ Aib2/I)-OH(Pheol16A/II)' at the N-terminus and $\mathrm{O}(\operatorname{Pro15A} / \mathrm{I})-\mathrm{N}(\mathrm{Phe} 1 \mathrm{~A} / \mathrm{II})$, OH(Pheol16A/I)-N(Phe1A/II), OH(Pheol16A/I)-N(Aib2A/II), OH(Pheol16B/I)-N(Phe1B/II) and $\mathrm{OH}(\mathrm{Pheol16B} / \mathrm{I})-\mathrm{N}(\mathrm{Aib} 2 \mathrm{~B} / \mathrm{II})$ at the C-terminus (/I and /II refers to molecule I and II, respectively). This higher number of hydrogen bonds seems to be a consequence of the increased flexibility at the termini that enables them to interact in various ways. Both disorder components take part in roughly the same number of hydrogen bonds and therefore have nearly equal occupancies. However, there is a reduction in the number of intermolecular hydrogen bonds involving the centre; only the $\mathrm{N}^{\varepsilon}(\mathrm{G} \ln 11 / \mathrm{I})-\mathrm{O}^{\delta}(\mathrm{Hyp} 13 \mathrm{~B} / \mathrm{II})$ " interaction can be detected. 


\section{BIOLOGICAL IMPLICATIONS}

\section{Membrane channels}

Several water and other solvent (mostly ethanol) molecules have been located during the refinement process. For cephaibol A and B, the solvent molecules seem to be equally distributed along the peptides, although some concentration is observed near the bend in the helix. On the contrary, for cephaibol $\mathrm{C}$ the solvent molecules seem to occupy exclusively the cavity between the two legs of the $\mathrm{X}$. This is in accordance with the [Leu1]zervamicin structure with the difference that the cephaibol $\mathrm{C}$ helix is not amphipatic, and suggests that even this channel would allow the transport of water molecules and ions when immersed in a biological membrane.

\section{Sequence variation and association properties}

Although closely related in chemical structure, the potency of cephaibol antibiotics shows considerable variation among the family members. Nevertheless, the assumption that variations in antibacterial action arises from significant differences in the three-dimensional structure can be rejected since their structures are very similar even at high resolution. However, comparison of intermolecular interactions reveals large differences in association properties. It seems that as the molecules become more flexible i.e. the amount of disorder in the peptide backbone increases, the number of interactions involving the bend region decreases, so that a new packing arrangement appears when changing from cephaibol $\mathrm{C}$ to cephaibol A. For cephaibol B, the centre takes part in only a handful of interactions, while the interactions involving the termini get stronger. It can be assumed that membrane-channelforming potency decreases in this order. It is interesting to note that the activity of cephaibols increase in the same order. Since cephaibols (as all peptaibol antibiotics) occur naturally as microheterogeneous mixtures, intermolecular interactions involving different members could lead to a large number of associates with different properties and account for their wide variety of action. In the case of gramicidin, where microheterogeneity also occurs, it was concluded that a small proportion of slightly different molecules might promote membrane integration and conversion into the conducting form and microheterogeniety was therefore not an undesired by-product of insufficient enzyme specificity, but had important biological role (Burkhart, Gassman et al., 1998), which might be the reason for microheterogeneity of peptaibols as well. 


\section{Implications for mechanism of action}

The mechanism of action of peptaibol antibiotics has not been unambiguously identified yet, but it seems to be widely accepted that antibiotic molecules constitute channels and thereby increase the permeability of biological membranes. Although conductivity measurements consistently prove the formation of ion channels, alternative techniques give contradicting results. For example, Aib residues in trichogin GA IV were substituted with a nitroxide spin label and the extent of insertion was assessed by the burial of the spin label using ESR and fluorescence quenching techniques (Peggion et al., 2003; Milov et al., 2003). Both approaches led to the conclusion that the helical axis was oriented in the plane of the bilayer and therefore no membrane channel formation was observed. More recently, using fluorescence and light-scattering measurements, the same authors concluded that trichogin was most likely to constitute pores in biological membranes (Stella et al., 2004).

The crystal structures of cephaibols suggest two possible action mechanisms. Cephaibol $\mathrm{C}$ is likely to constitute membrane channels, while cephaibol A and B probably act as ion carriers. A crystal structure in a lipid bilayer (e.g. cubic lipid phase, bicelles) may prove useful in establishing the mode of interaction with the membrane, as well as structure determination of cephaibol D and $\mathrm{E}$ that would complete the systematic investigation of the cephaibol series.

It seems therefore likely that peptaibols exhibit a variety of interactions with the cell membrane. In addition, their biological action is not limited to antibacterial potency, as peptaibols show other innovative phenomena like the induction of hypothermia and potential neuroleptic effects. The investigation of this secondary metabolite class therefore cannot be regarded as near to completion. These peptides seem to be occurring in many more species than previously thought, and it can be assumed that they have important biological functions (Degenkolb et al., 2003). 


\section{CRYSTAL STRUCTURE OF FEGLYMYCIN}

\section{INTRODUCTION}

Feglymycin is a naturally occurring peptide isolated from Streptomyces sp DSM 11171. The chemical structure of the peptide was elucidated using NMR-spectroscopy and mass spectrometry. Feglymycin was found to strongly inhibit the formation of HIV syncytia in vitro, but the molecular mechanism of antiviral action has not been investigated yet. A weak antibacterial activity against Gram-positive bacteria was also reported (Vértesy et al., 1999).

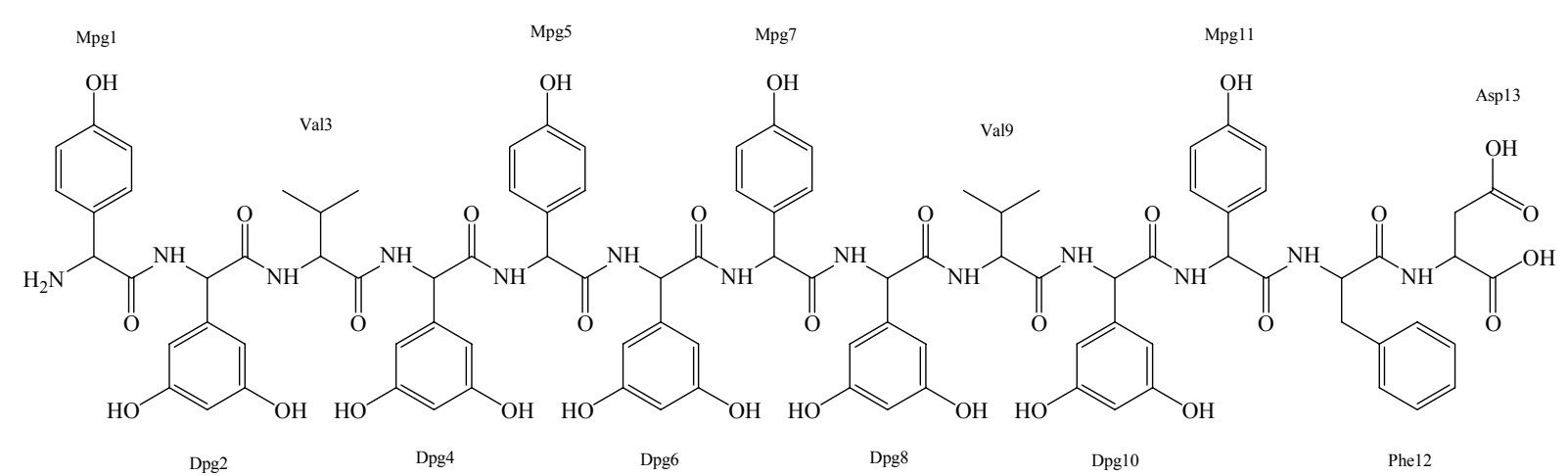

Figure 4.1

Chemical structure of feglymycin.

Feglymycin contains a high percentage of unusual amino acids: out of the 13 residues in the sequence, nine are either 4-hydroxyphenylglycine or 3,5-dihydroxyphenylglycine (Figure 4.1). Based on its chemical structure and biological activity, feglymycin constitutes a new group among antibiotics. Structural relatives include complestatin (Kaneko et al., 1989), the complestatin-related chloropeptins (Tanaka et al., 1997) and certain retrovirus protease inhibitors (RPI-856 A, B, C and D; Asano et al., 1994). These substances are all peptides that vary in length and contain at least one of the unusual hydroxyphenylglycine residues (Figure 4.2). Additional similar properties are that they are all produced by diverse Streptomyces $\mathrm{sp}$ and inhibit various steps of the replication of HIV. Complestatin and chloropeptins were found to prevent the binding of the gp120 viral protein to CD4 cellular receptor, while RPI-856 A, B, C and D inhibit the HIV protease with varying affinity. Moreover, the hydroxyphenyl moiety seems to have additional biologically interesting activities. This group 


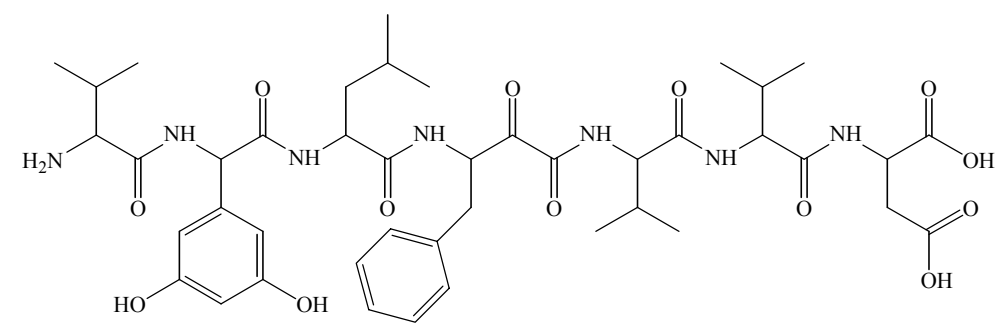

\section{Figure 4.2}

Chemical structure of RPI-856 A and B, which differ in the chirality of the $\beta$-methine carbon in the 3-amino-2-oxo-4-phenylbutyric acid residue.

readily undergoes oxidation and yields the corresponding quinone, which is a nuceloside analogue and therefore a potential substrate for DNA and RNA polymerases. A large percentage of anti-HIV agents isolated from natural sources also contains a 1,3-hydroxyphenyl group or a similar structural element in a condensed ring system. These compounds were shown to inhibit the HIV reverse transcriptase with varying efficiency, and in some cases additional antiviral activity has also been reported $(\mathrm{Ng}$ et al., 1997; Vlietnick et al., 1998; Matthée et al., 1999). For example, michellamine (Figure 4.3), a compound isolated from a rain forest plant, was initially identified as a potent inhibitor of the HIV reverse transcriptase (Boyd et al., 1994). In later studies, however, it also was found to inhibit cellular fusion and syncytium formation (McMahon et al., 1995). It seems therefore plausible that feglymycin might also possess multiple action mechanisms as an anti-HIV agent.

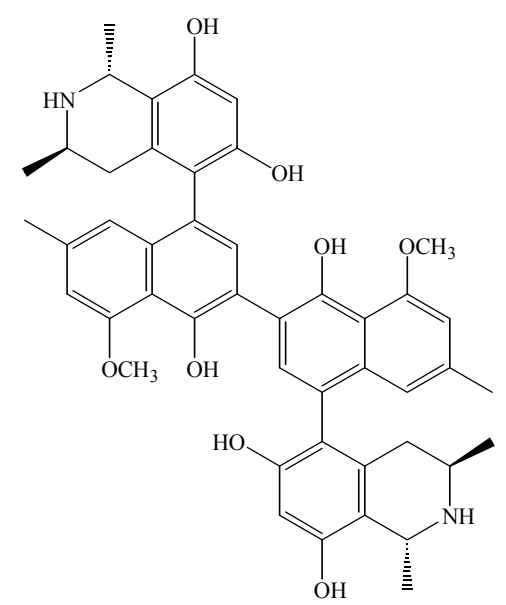

\section{Figure 4.3}

Chemical structure of michellamine B. 


\section{STRUCTURE DETERMINATION}

\section{Crystallisation}

Feglymycin is a white powder that is moderately soluble in water and well soluble in alcohols. As feglymycin was available only in a limited amount $(77 \mathrm{mg})$ and crystallisation conditions had not yet been established, it seemed reasonable to crystallise it with the hanging drop method, in which the substance used per crystallisation trial is negligible in comparison with batch methods commonly employed to crystallise small molecules. An adequate amount of the peptide was dissolved in ethanol:water=1:2 mixture to a concentration of $20 \mathrm{mg} / \mathrm{ml}$ and $2 \mu \mathrm{l}$ aliquots were employed in crystallisation trials at room temperature with the Hampton Crystal Screen I kit (Jancarik \& Kim, 1991). Crystals appeared in two conditions:

(a) Condition 8: $0.1 \mathrm{M} \mathrm{Na}$-cacodylate/cacodylic acid $\mathrm{pH}=6.5,30 \%$ isopropanol, $0.2 \mathrm{M}$ sodium citrate. Small needles were obtained.

(b) Condition 36: $0.1 \mathrm{M}$ Tris/Tris $\mathrm{HCl} \mathrm{pH}=8.5,8 \%$ PEG8000. Crystals having hexagonal bipyramidical morphology were grown.

These two conditions were optimised independently, and diffraction quality crystals (with dimensions up to $0.5 \cdot 0.3 \cdot 0.2 \mathrm{~mm}^{3}$ ) were obtained by:

(a) mixing $2 \mu \mathrm{l}$ of $40 \mathrm{mg} / \mathrm{ml}$ feglymycin in ethanol:water=1:4 with $2 \mu \mathrm{l}$ of either $0.1 \mathrm{M}$ Na-cacodylate/cacodylic acid $\mathrm{pH}=6.5,30 \%$ isopropanol and $0.25 \mathrm{M}$ Na-citrate or $0.25 \mathrm{M} \mathrm{Na}$-citrate/citric acid $\mathrm{pH}=6.5$ and $30 \%$ isopropanol at $4{ }^{\circ} \mathrm{C}$. Crystals appeared within a month (crystal form II, Figure 4.4).

(b) mixing $4 \mu \mathrm{l}$ of $10 \mathrm{mg} / \mathrm{ml}$ feglymycin in ethanol:water=1:4 with $2 \mu \mathrm{l}$ of $0.1 \mathrm{M}$ Tris/ Tris $\mathrm{HCl} \mathrm{pH}=8.4$ and 4\% PEG8000 at room temperature. Crystal growth took about two weeks (crystal form I, Figure 4.4).

\section{Measurement}

For measurement, a crystal was taken out from the drop with a suitably sized loop and transferred into a droplet of the cryoprotectant solution containing $0.2 \mathrm{M}$ Tris/Tris $\mathrm{HCl}$, $4 \%$ PEG8000 and 25\% glycerol for $\mathbf{I}$ and $0.3 \mathrm{M}$ Na-citrate/citric acid $\mathrm{pH}=6.5$, $30 \%$ isopropanol and 20\% 1,2-propanediol for II. After a short soaking time, crystals were taken out with the loop and shock frozen in a cold nitrogen stream operating at $100 \mathrm{~K}$ (when crystallisation was performed at room temperature) or by dipping the loop into liquid nitrogen (for crystals grown at $4{ }^{\circ} \mathrm{C}$ ). 


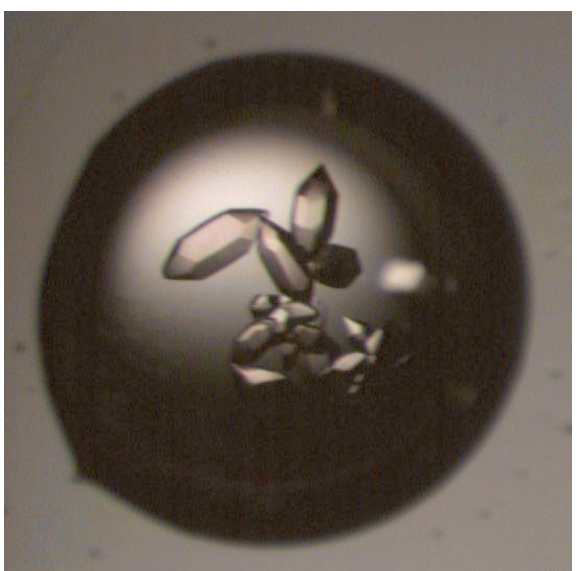

Crystal form I

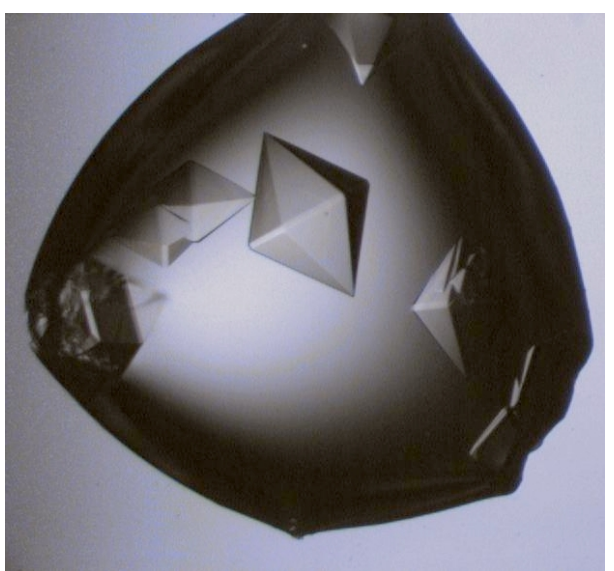

Crystal form II

Figure 4.4

Crystal morphologies of feglymycin.

Crystals were then characterised by collecting datasets on a Bruker rotating anode equipped with Osmic focusing mirrors and a SMART6000 4K CCD detector. Images were indexed with the program Proteum and data were processed using SAINT and SADABS (Bruker Nonius, 2002). XPREP was employed for space group determination and for merging. Data collection statistics are summarised in Table 4.1.

\begin{tabular}{|c|c|c|c|c|}
\hline & \multicolumn{2}{|c|}{ Crystal form I } & \multicolumn{2}{|c|}{ Crystal form II } \\
\hline & $6 / \mathrm{m}$ & $6 / \mathrm{mmm}$ & $6 / \mathrm{m}$ & $6 / \mathrm{mmm}$ \\
\hline Unit cell parameters $(\AA)$ & \multicolumn{2}{|c|}{$\mathrm{a}=\mathrm{b}=83.544, \mathrm{c}=35.734$} & \multicolumn{2}{|c|}{$\mathrm{a}=\mathrm{b}=60.302, \mathrm{c}=83.748$} \\
\hline$<\left|E^{2}-1\right|>$ & \multicolumn{2}{|c|}{0.730} & \multicolumn{2}{|c|}{0.960} \\
\hline Space group & $\mathrm{P}_{5}$ & $\mathrm{P}_{5} 22$ & $\mathrm{P} 6_{4}$ & $\mathrm{P}_{6} 22$ \\
\hline No. of reflections & 578199 & 606418 & 484869 & 484869 \\
\hline No. of unique reflections & 57985 & 34003 & 34149 & 18476 \\
\hline Resolution $(\AA)$ & $1.10(1.20-1.10)$ & $1.06(1.15-1.06)$ & $1.40(1.50-1.40)$ & $1.40(1.50-1.40)$ \\
\hline Completeness & $100 \%(100 \%)$ & $99.7 \%(98.7 \%)$ & $99.8 \%(99.1 \%)$ & $99.8 \%(99.2 \%)$ \\
\hline Redundancy & $9.97(4.64)$ & $17.78(8.11)$ & $14.17(10.95)$ & $26.20(20.71)$ \\
\hline$<I / \sigma(I)>$ & $25.31(3.06)$ & $31.67(3.62)$ & $21.50(3.49)$ & $29.18(4.79)$ \\
\hline$R_{\text {int }}$ & $0.0408(0.4482)$ & $0.0488(0.5682)$ & $0.0634(0.6665)$ & $0.0640(0.6811)$ \\
\hline
\end{tabular}

\section{Table 4.1}

Data collection statistics for feglymycin crystal forms. Statistics are included for the real and also for the apparent space group. $R_{\text {int }}=\Sigma|I-<I>| / \Sigma I$. 

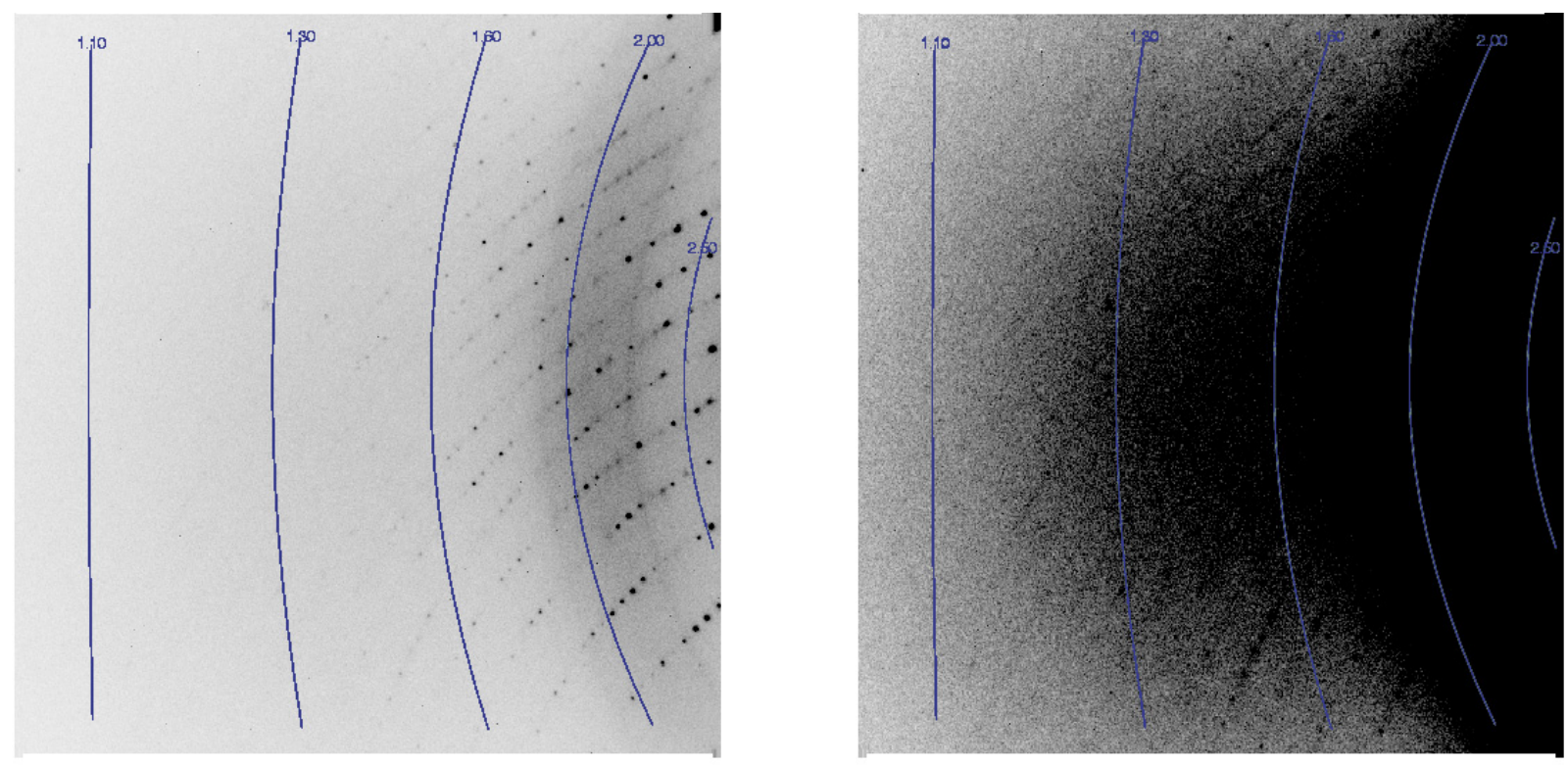

\section{Figure 4.5}

Diffraction from crystal form I (same frame, different contour levels). Based on this image (frame no. 1 , which actually has a low scale factor) a resolution cutoff about $1.4 \AA$ may be justified, but a low-background detector, high redundancy and careful data collections gives intensities to $1.1 \AA$.

Crystal form I was found to have a hexagonal unit cell with dimensions $\mathrm{a}=\mathrm{b}=83.54 \AA$ $\mathrm{c}=35.73 \AA$ and examination of the systematic absences together with the Laue-symmetry suggested that the space group was either $\mathrm{P} 6_{1} 22$ or $\mathrm{P} 6_{5} 22$. A dataset consisting of three $180^{\circ}$ $\omega$-scans at $2 \Theta=64^{\circ}$ for the high-resolution data $(1.05-2.0 \AA)$ and three $180^{\circ} \omega$-scans at $2 \Theta=30^{\circ}$ for the low-resolution shells (to $1.55 \AA$ ) was collected. After processing, it was indicated by the statistics that the crystal, although weakly, diffracted to atomic resolution, as in the highest resolution shell (1.15-1.06 $\AA$ ) the average intensity was marginally higher $(3.62 \sigma)$ than the limit of statistical significance $(3 \sigma)$. This was probably a joint benefit of the comparatively high redundancy, the thin-sliced data collection strategy and the highly sensitive low-background detector as on the frames very few reflections above $1.2 \AA$ were discernible (Figure 4.5).

Crystal form II also belongs to the hexagonal system with cell dimensions $a=b=60.30 \AA$ $\mathrm{c}=83.75 \AA$ and systematic absences indicated $\mathrm{P}_{2} 22$ or $\mathrm{P}_{4} 22$ as possible space groups. The crystal diffracted to about $1.8 \AA$ on the home source and therefore a higher resolution dataset on the BL1 beamline at BESSY was also collected. To avoid overloads in the lowresolution shells, the data were collected in two runs with considerably shorter exposure times for the low-resolution frames. The data extends to $1.4 \AA$ and was integrated with HKL2000 
and scaled with SADABS. It is noteworthy that datasets of this crystal had an unusual $\left|E^{2}-1\right|$ value $(0.960)$ more common for centrosymmetric space groups $(0.968)$.

\section{Structure solution}

\section{Crystal form I}

Structure solution of antibiotic molecules is usually complicated by the presence of several copies of the molecule in the asymmetric unit, which becomes too big for traditional direct methods even if atomic resolution data were available, and a markedly different structure from everyday proteins, which decrease the efficiency of molecular replacement and the traditional heavy atom derivatisation, since the required interaction surfaces are missing. For that reason, a currently topical method for derivatising the crystal with non-specific anomalous scatterers, namely an iodide soak was performed first by soaking a crystal in about $0.5 \mathrm{M} \mathrm{KI}$, but deterioration of diffraction quality (maximum resolution worse than $3 \AA$ ) and no significant anomalous signal in the dataset indicated that this method is not applicable for this crystal.

As atomic resolution data were available, ab initio solution using direct methods was the method of choice. However, the absence of heavy atoms and the relatively large unit cell that could accommodate up to 1000 atoms did not suggest a quick solution, as the largest equal-atom structure solved so far with SHELXD (Sheldrick et al., 2001) contained only a few more than 700 atoms and the process took about six months CPU time on a Pentium 200 processor. Two different strategies were employed in parallel:

(a) search for a considerable portion of the atoms (FIND 600) and then perform peaklist optimisation (PLOP 800 1000).

(b) find only a small percentage of the atoms (FIND 200) and do several cycles of peaklist optimisation (PLOP 300450675 1000) for the promising ones (TEST 25 5).

Strategy (a) was slower, it required about one hour for a try, but it was expected that the efficiency of the dual-space recycling in the search compensated for the increased computational demand. In strategy (b), which required only 9 minutes/try, the increased speed was exploited to test a higher number of starting atom sets when searching for the solution.

After one week CPU-time on a $2 \mathrm{GHz}$ Intel Xeon processor, SHELXD run (b) gave a solution, although it could not be discriminated from non-solutions based on the standard figure-of-merit final correlation coefficient, which should be above 65 in order to consider the structure to be solved with high probability. However, both the $\mathrm{CC}$ and the CCweak 
figures-of-merit showed a good discrimination of the solution from the non-solutions. Computer graphics examination of the solution with the program XP revealed a chemically sensible peptide structure with the expected amino acids and confirmed the success. Later the presence of perfect merohedral twinning was recognised that may account for the low correlation coefficient value. As the screw axis was undoubtedly identified previously as being a $6_{1} / 6_{5}$, obtaining the twin law was straightforward. However, direct solution with the twin law and the lower symmetry space group was unsuccessful, probably because of the size of the structure and the lower resolution (merging in $6 / \mathrm{m}$ reduced the redundancy and justified a cutoff at $1.1 \AA)$.

\section{Crystal form II}

As the data extended to only sub-atomic resolution and the structure of crystal form $\mathbf{I}$ was available at that time, molecular replacement was tried. The program EPMR (Kissinger et al., 1999) was employed with a feglymycin dimer as the search model and data between 10 and $3.2 \AA$ were used. EPMR was selected as the search model was relatively small and division of the six-dimensional search for a rotational and a translational one is no longer valid in such a case. Assuming 50\% solvent content found at crystal form I, two dimers were expected in the asymmetric unit. As the hand of the space group is not determined and the search model is chiral, solution was attempted in $\mathrm{P}_{2} 22$ and $\mathrm{P} 6_{4} 22$. In $\mathrm{P}_{2} 22$ no promising results were obtained, while in $\mathrm{P}_{4} 22$ EPMR found the first dimer (dimer 1) with a high

(a)

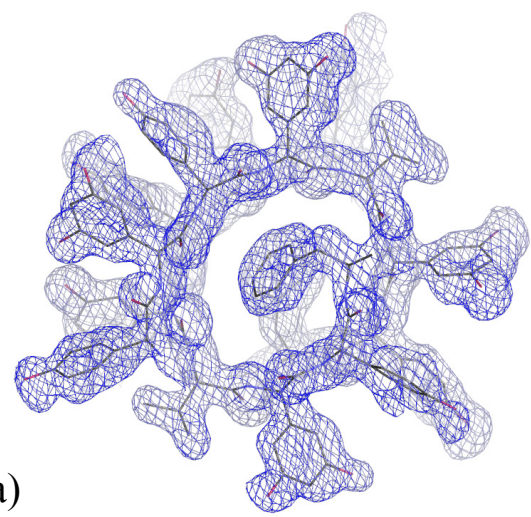

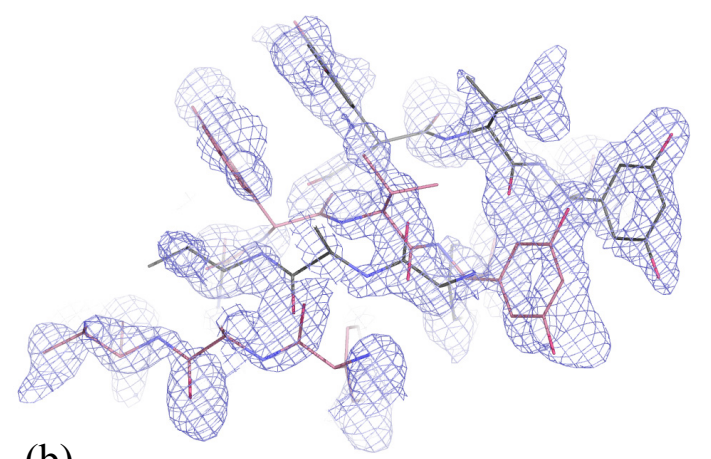

(b)

\section{Figure 4.6}

Electron density from SHELXE for crystal form II in P6 22. (a) Dimer 1 is clearly seen in the density and even the side chains are recognisable, which were not included in the starting model. (b) In contrast, electron density on dimer 2 is not directly interpretable and is accounted for by two overlapped molecules. The model is taken from the final refinement, but symmetry equivalents were generated according to $\mathrm{P}_{4} 22$ symmetry. 
success rate and good discrimination, but addition of the second molecule (dimer 2) did not improve the figures-of-merit. Examining the packing of the two dimers revealed that while dimer 1 packed in a chemically sensible way, dimer 2 had several close contacts with itself. As the resolution of the data is superior to normal proteins and the solvent content is not too low, SHELXE (Sheldrick, 2002) was expected to produce an excellent map even when starting from a partial solution. For that purpose, dimer 2 was removed from the model, and dimer 1 was used as a starting seed. The obtained map was by no means clearer than the one calculated from the model using SigmaA-weights (Figure 4.6). This suggested the presence of perfect merohedral twinning, and based on packing violations, the same twin law was found as for crystal form I. It is interesting to note that the characteristic indication for twinning, namely the $\left|E^{2}-1\right|$ value in that case is actually too high and is not typical for a twinned structure. EPMR was then re-run in the lower symmetry space group, but could locate two dimers corresponding to dimer 1 in $\mathrm{P}_{4} 22$. In another try, this tetramer was held fixed and the missing two dimers were searched as one tetramer. This approach led to a solution with respectable statistics.

\section{Model building and refinement}

Both structures were refined against $F_{o}{ }^{2}$ with no intensity cutoff using SHELXL, which can handle twinning and is generally recommended for high-resolution structures. Detwinned intensity data output by SHELXL (Sheldrick \& Schneider, 1997) were used to calculate and inspect $2 m F_{o}-D F_{c}$ and $F_{o}-F_{c}$ type maps using the program XtalView (McRee, 1999) for manipulation of atoms. Throughout the refinement, bond length, bond angle and chiral volume restraints were imposed, which were taken from the Engh and Huber library (Engh \& Huber, 1991) for aspartate, phenylalanine and valine or generated from the Cambridge Structural Database (Allen, 2002) for 4-hydroxyphenylglycine and 3,5-dihydroxyphenylglycine. For the two enantiomers of 4-hydroxyphenylglycine present in the structure, the same restraints were employed except for the sign of the chiral volume restraint, which was inverted. Planarity restraints were applied to peptide bonds and aromatic side chains. Solvent atoms were added using SHELXWAT and also manually. All nonhydrogen atoms were refined anisotropically with suitable rigid bond and similarity restraints. Approximately isotropic restraints were also employed for solvent water molecules. Hydrogen atoms were included in the later stages of refinement. Refinement statistics are listed in Table 4.2. 


\section{Crystal form I}

Refinement was initiated in the space group $\mathrm{P}_{5} 22$ (chirality determined by assigning the common amino acids to have L-configuration found by HPLC on chiral medium). After two rounds of B-value refinement, all peptide atoms could be located. The structure consisted of three peptide chains in one and a half dimers, a crystallographic twofold axis generating the second half of the dimer. The $R$-values were $31 \%$ and $33 \%$ for $R_{1}$ and $R_{\text {free, }}$, respectively, which are suspiciously higher values than expected for a complete structure. It was realised at this stage that although a dimer nearly obeyed twofold symmetry, the side chain of Phe12 residue did not and would always collide with its symmetry equivalent. This finding suggests that this twofold axis cannot be crystallographic as it would always imply the presence of disorder, the space group is therefore only $\mathrm{P}_{5}$ that is twinned by a twofold rotation perpendicular to $\mathbf{c}$ and contains three dimers in the asymmetric unit and a noncrystallographic twofold axis. The nearly perfect non-crystallographic twofold also offered an explanation to the severe non-planarity of the benzene rings that was significant at such a high resolution. Refinement in $\mathrm{P}_{5}$ gave lower $R$-values $\left(R_{1}=27.03 \%\right.$ vs. $\left.31.00 \%\right)$ and solved the
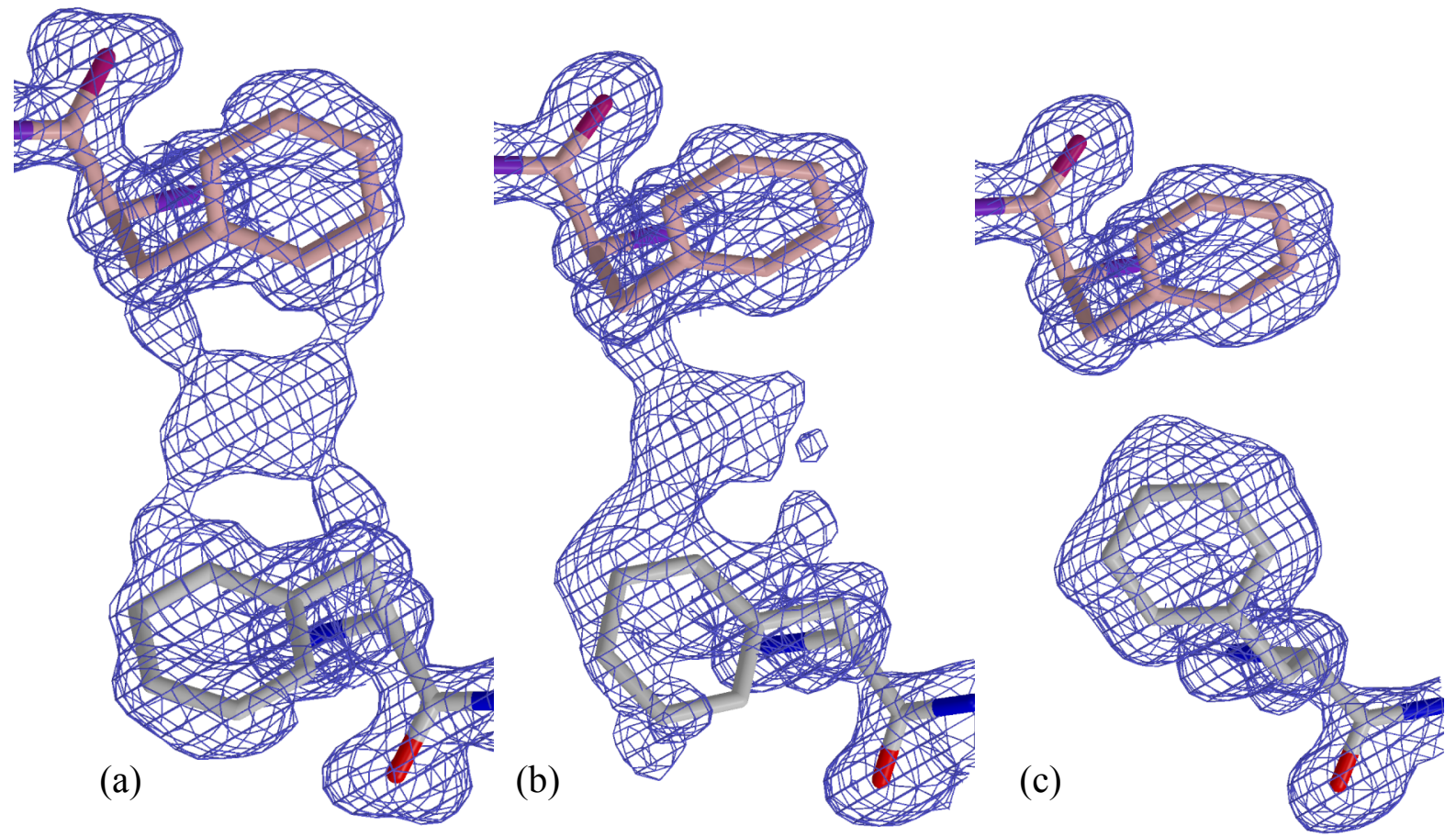

\section{Figure 4.7}

View of the sidechain of Phe12 and its symmetry equivalent along a crystallographic twofold axis.

(a) In $\mathrm{P}_{5} 22$ there is density in between, but fitting the atoms would involve a disorder, $R_{1}=31.0 \%$.

(b) After the first refinement in $\mathrm{P} 6_{5}$, the density is polarised towards one of the residues (no longer symmetry equivalents), $R_{1}=27.0 \%$. (c) After adjustment, clear density appears on the sidechains, $R_{1}=26.9 \%$. 
problem of the side chain conformation for the aforementioned phenylalanine residue (Figure 4.7). Comparison of $K$-values $\left(K=<F_{o}>/<F_{c}>\right)$ as a function of reflection intensity as output by SHELXL showed a more common, flat behaviour for the refinement in $\mathrm{P} 6_{5}$, while when refining in $\mathrm{P}_{5} 22$ without twinning resulted in a $K$-value significantly higher for the weakest reflections than the average. Refinement in $\mathrm{P}_{5}$ without the twin law resulted in a significant increase in $R$-values. Despite the usual $\left|E^{2}-1\right|$ value, the assumption of twinning was considered to be justified and refinement was carried on in $\mathrm{P}_{5}$. The final model contains 78 residues, several PEG units and 77 water molecules, and with 1026 non-hydrogen atoms it is by far the largest equal-atom structure solved with ab initio direct methods so far.

\section{Crystal form II}

As the presence of twinning had already been established in the structure solution stage, refinement was initiated taking the twin law into account. The availability of the complete structure from crystal form I simplified model building to completing the solvent structure since the conformation of feglymycin in the two crystal forms was found to coincide nearly exactly. In the electron density maps the occurrence of significant radiation damage can be seen, especially at the aspartate residues, and zero-dose extrapolation improves the map in these regions. The final model contains 104 residues, 4 isopropanol and 106 water molecules.

\begin{tabular}{lll}
\hline & \multicolumn{1}{c}{ Crystal form I } & \multicolumn{1}{c}{ Crystal form II } \\
\hline Resolution range $(\AA)$ & $72.35-1.10$ & $28.37-1.40$ \\
$R_{1}[\mathrm{~F}>4 \sigma(\mathrm{F}) /$ all data $]$ & $0.1434 / 0.1689$ & $0.1590 / 0.1764$ \\
$R_{\text {free }}[\mathrm{F}>4 \sigma(\mathrm{F}) /$ all data $]$ & $0.1674 / 0.1950$ & $0.1957 / 0.2193$ \\
No. of parameters & 9811 & 11147 \\
No. of restraints & 13414 & 14594 \\
No. of peptide atoms & 828 & 1104 \\
No. of non-H atoms & 1026 & 1221 \\
Solvent content & $51.2 \%$ & $52.6 \%$ \\
Twin ratio & 0.494 & 0.500 \\
R.m.s.d. from ideal geometry & & \\
\multicolumn{1}{c}{ Bond lengths $(\AA)$} & 0.022 & 0.017 \\
\multicolumn{1}{c}{ Angle distances $(\AA)$} & 0.045 & 0.037 \\
\multicolumn{1}{c}{ Common plane $(\AA)$} & 0.290 & 0.293 \\
\hline
\end{tabular}

\section{Table 4.2}

Refinement statistics. 


\section{STRUCTURE DESCRIPTION}

\section{Amino acid residues}

As feglymycin contains unusual amino acids and the chemical structure was established with NMR and MS techniques, nothing was known about the chirality except for the fact that the common amino acids aspartate, phenyalanine and valine were identified to have L-configuration. The X-ray structure revealed that all the 3,5-dihydroxyphenylglycine (Dpg) residues have D-configuration, the first 4-monohydroxyphenylglycine (Mpg) residue possesses D-configuration, while the other three Mpg residues have L-configuration. The sequence therefore can be written as:

\section{D-Mpg-D-Dpg-L-Val-D-Dpg-L-Mpg-D-Dpg-L-Mpg-D-Dpg-L-Val-D-Dpg- L-Mpg-L-Phe-L-Asp}

Feglymycin thus consists of alternating D,L-amino acids with certain deviations from this regularity near the termini. This pattern of amino acids with alternating chirality is not unusual among secondary metabolites and the extensively studied antibiotic gramicidin isolated from Bacillus brevis exhibits the same alternation with the difference that for gramicidin this pattern runs along the sequence without exceptions (Sarges \& Witkop, 1956).

\section{Dimeric structure}

Feglymycin was found to form dimers in both crystal forms. The dimers are of the right-handed double-stranded antiparallel double $\beta$-helix type and contain about 9 residues/turn (Figure 4.8). This finding is consistent with all known X-ray structures of gramicidin, where dimer formation is also a characteristic feature. However, in the case of gramicidin, the helix rise is 5.6 residues/turn for the uncomplexed structure (Langs, 1988) and 7.2 residues/turn when crystallised as a $\mathrm{Cs}^{+}$-complex (Burkhart, Li et al., 1998). An additional difference is the presence of two phenylalanine sidechains inside the feglymycin channel, while the gramicidin pore is empty (Figure 4.9). Nevertheless, exhibiting such extensive similarities, it is probably justified to classify feglymycin as a structural analogue of gramicidin.

\section{Dimer formation}

The feglymycin dimer is a very compact molecule that is stabilised by a huge number of secondary interactions. The formation of the dimer can be visualised as follows. In the first 


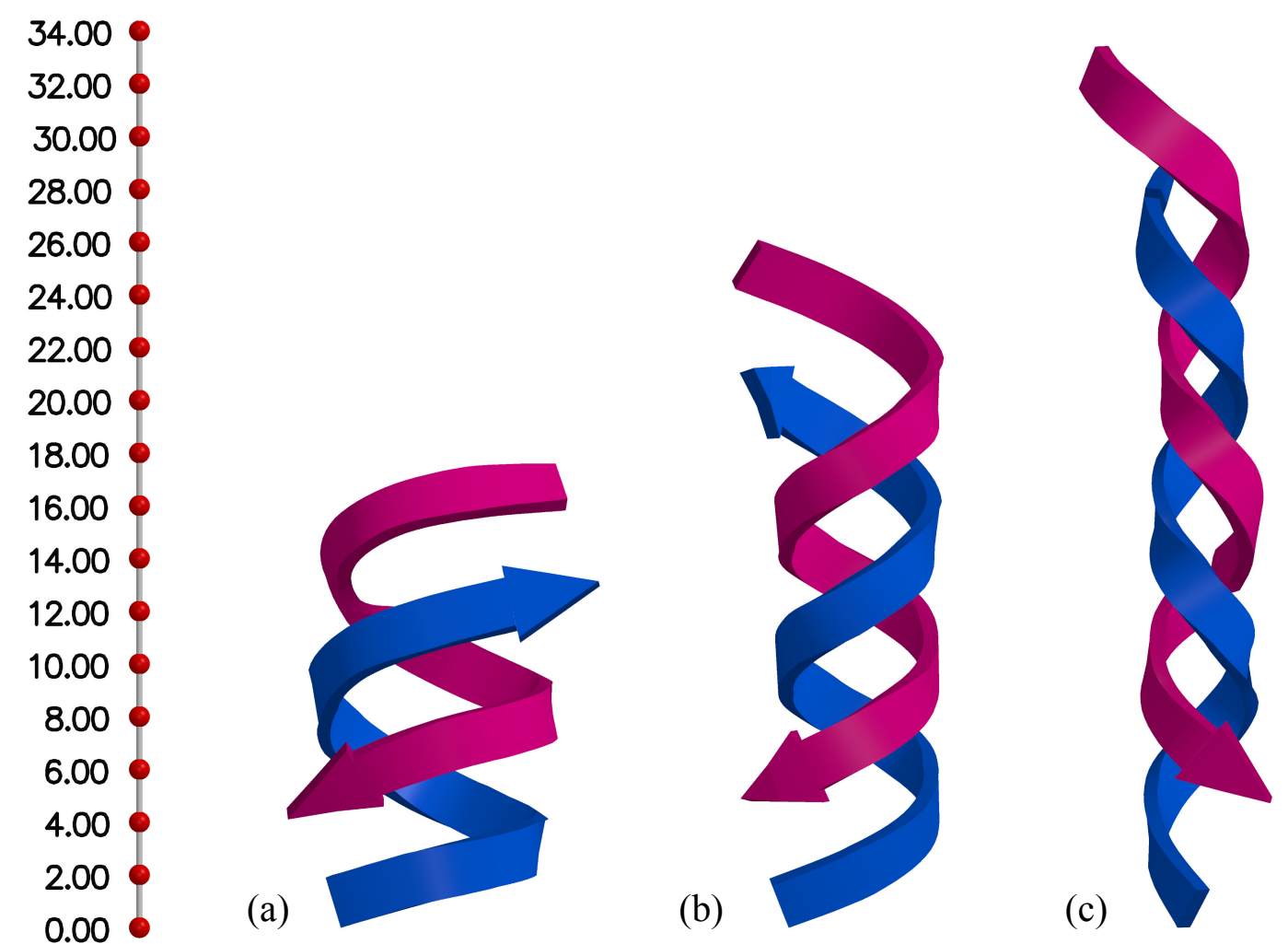

Figure 4.8

Schematic representation of the size and structure of feglymycin (a), gramicidin- $\mathrm{Cs}^{+}$(b) and uncomplexed gramicidin (c) dimers. (a) and (b) are right-handed helices, while (c) is left-handed. The absolute scale is shown by the ruler on the left in $\AA$ units.

step, two molecules of feglymycin associate in an antiparallel fashion to form a two-stranded $\beta$-sheet. This $\beta$-sheet is unusual by the fact that all sidechains are pointing to the same side of the strand because of the regular alternate chiral arrangement of the residues and has a considerable sterical tension originating from close contacts between sidechain atoms, which exerts a force on the backbone and tries to bend it. The backbone gives way to the pressure and assumes a helical conformation. In the last step, a conformational rearrangement of the sidechains to form interstrand hydrogen bonds further stabilises the dimer.

\section{Dimer symmetry}

In case of gramicidin, the dimer both in the uncomplexed and the $\mathrm{Cs}^{+}$-complexed form was found to exhibit noncrystallographic symmetry with the mainchain atoms being nearly exactly related by a twofold axis and some minor deviations at certain sidechains. As a homologue, feglymycin was also expected to show twofold symmetry.

To get an unbiased comparison of monomers in a dimer, rigid sets of atoms were identified with the program ESCET (Schneider, 2002) among all molecules in crystal form I. 


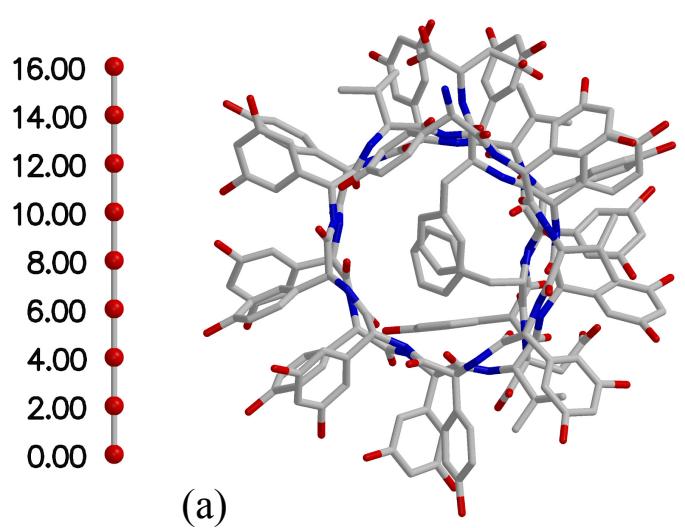

(b)
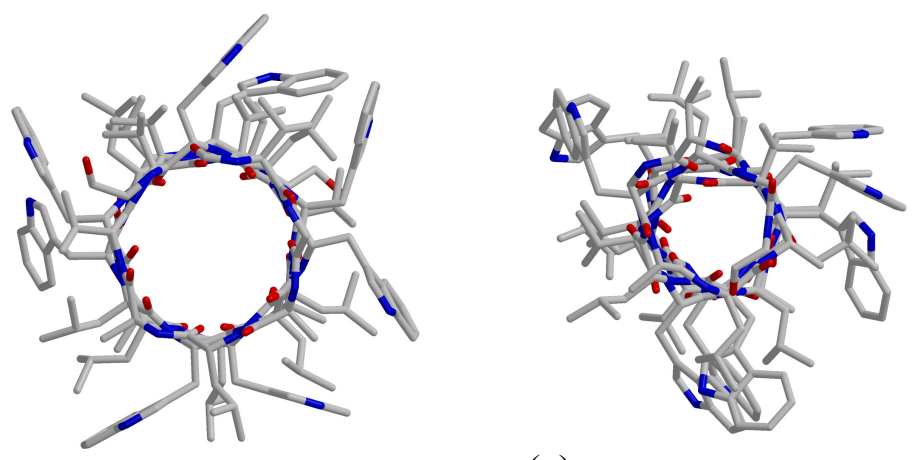

(c)

Figure 4.9

View along the helix axis for feglymycin (a), gramicidin- $\mathrm{Cs}^{+}$(b) and for the uncomplexed gramicidin (c) dimer. Each helix has a different turn: (a) belongs to the $\beta^{9.0}$-family, (b) to the $\beta^{7.2}$-family and (c) to the $\beta^{5.6}$-family and accordingly differ in pore diameter. The ruler on the left is in $\AA$ units.

As crystal form II was solved using molecular replacement with a dimer from crystal form I, it was found that all four dimers in crystal form II closely resembled the structure of the model and were omitted from the analysis. The rigid atoms in the six monomers present in crystal form I were then fitted onto each other and compared using XtalView (Figure 4.10).

The six monomers were found to be significantly different within the error of the analysis. Based on their conformation, the monomers can be divided into two classes (A and B), the main difference being the mainchain conformation for residues 12 and 13. A dimer is built up by one monomer having class $\mathrm{A}$ and one exhibiting class $\mathrm{B}$ conformation and this implies that the feglymycin dimer is less symmetric than the one in gramicidin. This
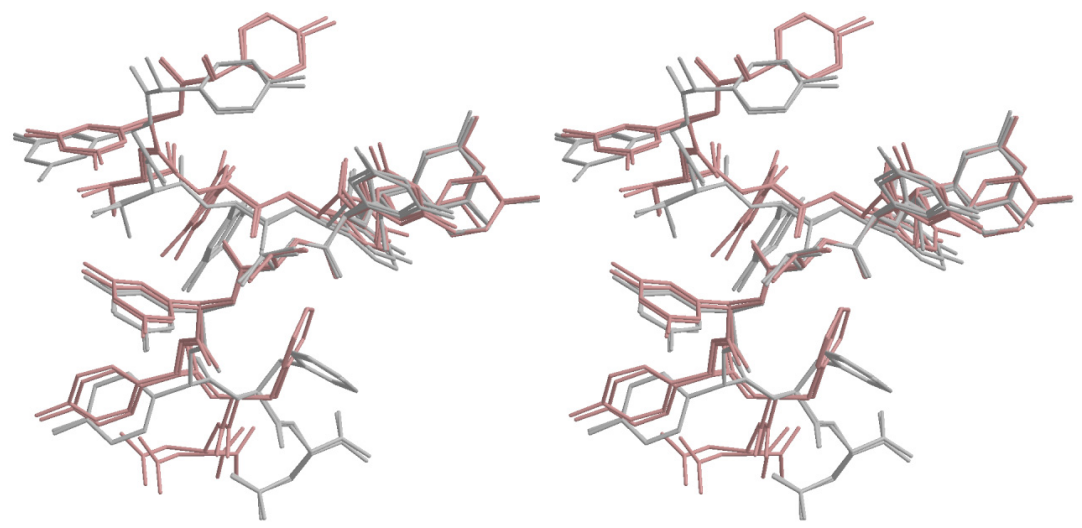

Figure 4.10

Stereo view of the fit of feglymycin monomers reveals two conformation classes (grey and redbrown). The $\mathrm{N}$-terminus is on the top, the $\mathrm{C}$-terminus and the neighbouring Phe12 is near the bottom. A fit of four monomers is displayed. 
difference may arise from the fact that feglymycin is not as regular concerning the chirality alternation as gramicidin, and the finding that the deviation occurs in a region where the regular alternation is broken seems to confirm the assumption. It is interesting to note that at the other terminus, where one residue violates the regularity, there is also a conformational diversity observed. The conformational flexibility exhibited by this residue may help in adaptation to the environment and therefore be important in stabilising the crystal lattice, which is supported by the finding that this residue is fundamentally involved in interdimer interactions.

\section{Secondary interactions}

The helical dimer present in the structure is stabilised by a number of hydrogen bonds. As the structure is fairly complex, for a sufficiently clear description a notation is introduced at this point that will be used throughout this chapter. Atoms are designated following PDB notation for conventional residues; for unconventional residues the numbering is shown in Figure 4.11. Independent dimers in the asymmetric unit will be distinguished by roman numbers. Monomers are denoted by capital letters A and B that refer to their conformation class, followed by the number of the dimer. If this number is omitted, the statement is meant generally and applies to all dimers. Apostrophes indicate symmetry equivalents; for those generated by a common symmetry operator the same notation is used. Thus, the hydrogen bonds can be classified as:

(a) "primary" hydrogen bonds between mainchain atoms of the two antiparallel chains. Interactions belonging to this category join the carbonyl oxygen and peptide nitrogen atoms of even-numbered residues of one chain with the even-numbered residues of the

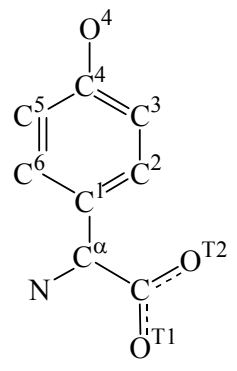

$\mathrm{Mpg}$<smiles>NC(C(=O)[O-])c1cc([O])cc(O)c1</smiles>

Dpg

\section{Figure 4.11}

Atom names for 4-monohydroxyphenylglycine (Mpg) and 3,5-dihydroxyphenylglycine (Dpg). 
other in an antiparallel fashion (Figure 4.12). Residues Dpg2-Phe12 are involved and as there are two interactions per residue, altogether 12 such hydrogen bonds are formed. An additional hydrogen bond $\mathrm{O}^{\delta 1}($ Asp13-A)-N(Mpg1-B) provides further strength to the dimer. However, there is no interaction between N(Mpg1-A)$\mathrm{O}^{\delta 1}($ Asp13-B), i.e. between the other termini, which is a consequence of the displacement of Asp13 in the class B chains. These hydrogen bonds are conserved in all independent dimers.

(b) "secondary" hydrogen bonds between mainchain atoms that are corollaries of the helical structure. On the other hand, these are interactions that stabilise the double helix. There are considerably less hydrogen bonds that belong to this class, as only the odd numbered residues between Val9-Asp13 participate (Figure 4.12). These involve 5 hydrogen bonds, namely O(Va19-A)-N(Asp13-B), N(Mpg11-A)-O(Mpg11-B), O(Mpg11-A)-N(Mpg11-B), N(Asp13-A)-O(Va19-B) and $\mathrm{O}^{\mathrm{T} 1}$ (Asp13-A)-N(Va19-B). It is noteworthy that the $\mathrm{N}(\mathrm{Val}-\mathrm{A})-\mathrm{O}^{\mathrm{T} 1}(\mathrm{Asp} 13-\mathrm{B})$ is not present since Asp13-B is further away. Two additional interactions between $\mathrm{O}(\mathrm{Va19}-\mathrm{A})-\mathrm{O}^{\mathrm{T1}}(\mathrm{Asp} 13-\mathrm{B})$ and the opposite direction $\mathrm{O}^{\mathrm{T1}}(\mathrm{Asp} 13-\mathrm{A})-\mathrm{O}(\mathrm{Val9}-\mathrm{B})$ strengthen the interaction further, which suggest that the termini are protonated. Since the bond distance in the first interaction is $3.6 \AA$, while in the second it is $3.3 \AA$, these interactions are rather weak. The helical

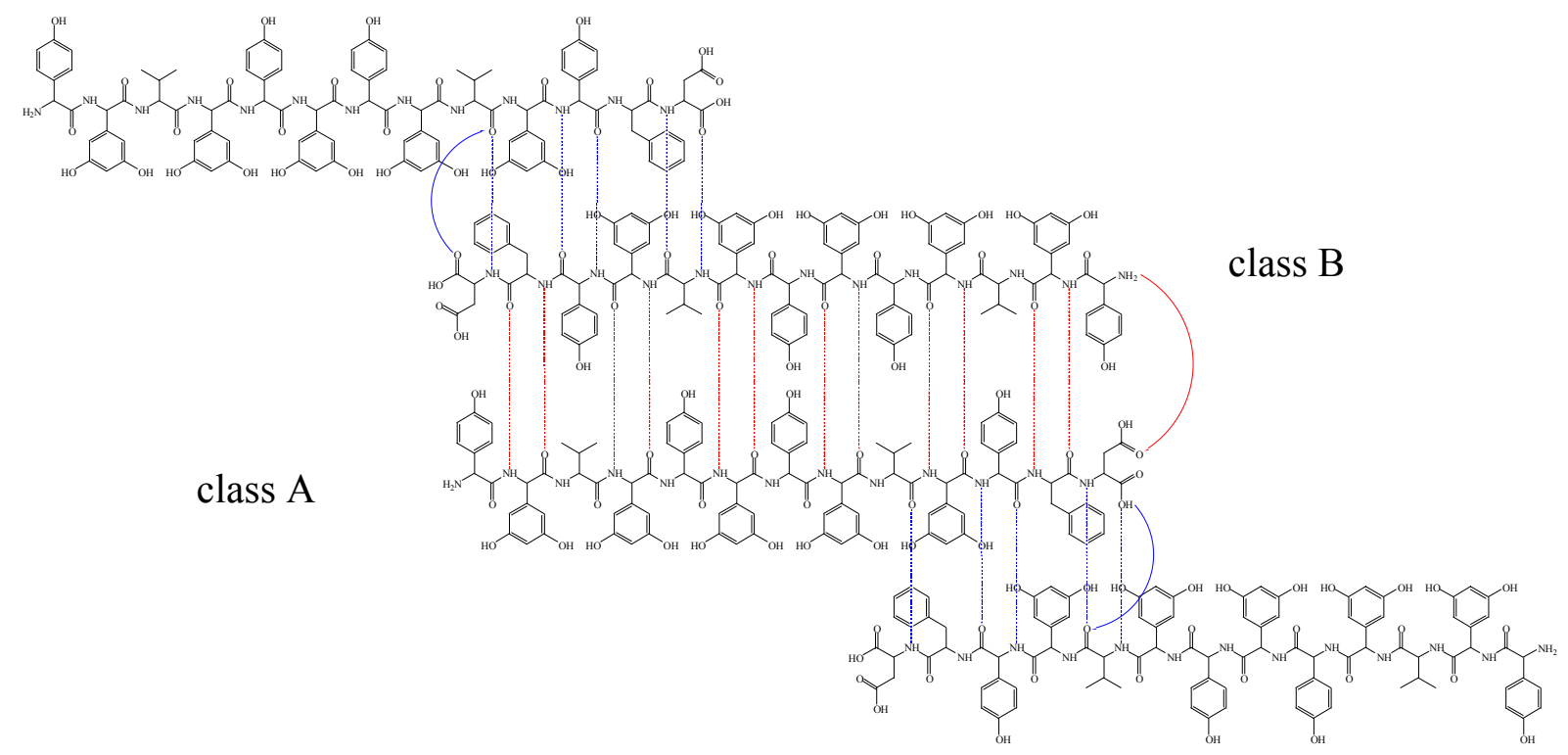

\section{Figure 4.12}

Hydrogen bonds within a feglymycin dimer. "Primary" hydrogen bonds (red) connect the two antiparallel chains, while "secondary" hydrogen bonds (blue) secure the helical structure. The "offset" of the helix is thus eight residues. 
structure is not stabilised by as many interactions as those that join the two chains, and can be expected to rearrange under favourable conditions. Such a process has already been described for gramicidin that forms a left-handed double helix when uncomplexed, but undergoes substantial conformational movement when a suitable metal atom is added to the solution and converts into a right-handed double helix (Burkhart et al., 1999). Little variation in these hydrogen bonds among independent dimers in the asymmetric unit can be observed, namely the $\mathrm{O}(\mathrm{Val9}-\mathrm{A})-\mathrm{O}^{\mathrm{T} 1}$ (Asp13-B) can be replaced by $\mathrm{O}(\mathrm{Val} 9-\mathrm{A})-\mathrm{O}^{\delta 1}(\mathrm{Asp} 13-\mathrm{B})$.

(c) hydrogen bonds that involve hydroxyphenyl moieties. Feglymycin possesses therefore two shells of hydrogen bonds: one is constituted by mainchain atoms and the other comprises hydrogen bonds between sidechain hydroxyls that are further apart from the helix axis (Figure 4.13). This multi-shell structure may provide unprecedented rigidity to the feglymycin dimer. The ordering of these bonds produces long-range hydrogenbonded chains on the surface of the molecules, and gives directionality to all Dpg residues. Four such chains can be discerned:

(i) $\mathrm{O}^{3}(\mathrm{Dpg} 2-\mathrm{A})-\mathrm{O}^{\delta 1, \delta 2}(\mathrm{Asp} 13-\mathrm{B}) \mathrm{O}^{\delta 2}-\mathrm{O}^{3}(\mathrm{Dpg} 10-\mathrm{A}) \mathrm{O}^{5}-\mathrm{O}^{5}(\mathrm{Dpg} 4-\mathrm{B})$,

(ii) $\mathrm{O}^{3}($ Dpg $4-\mathrm{A})-\mathrm{O}^{5}(\mathrm{Dpg} 10-\mathrm{B}) \mathrm{O}^{3}-\mathrm{HOH}-\mathrm{O}^{3}(\mathrm{Dpg} 2-\mathrm{B})-\mathrm{O}^{\delta 2}(\mathrm{Asp} 13-\mathrm{A})$,

(iii) $\mathrm{O}^{3}\left(\right.$ Dpg6-A) $-\mathrm{O}^{5}(\mathrm{Dpg} 8-\mathrm{B}) \mathrm{O}^{3}-\mathrm{HOH}-\mathrm{O}^{\mathrm{T}}(\mathrm{Asp} 13-\mathrm{A})$ and

(iv) $\mathrm{O}^{3}\left(\right.$ Dpg8-A) $-\mathrm{O}^{3}($ Dpg6-B).

As atoms involved in these interactions are on the surface and most susceptible to packing interactions, the hydrogen-bonding pattern shows considerable variation
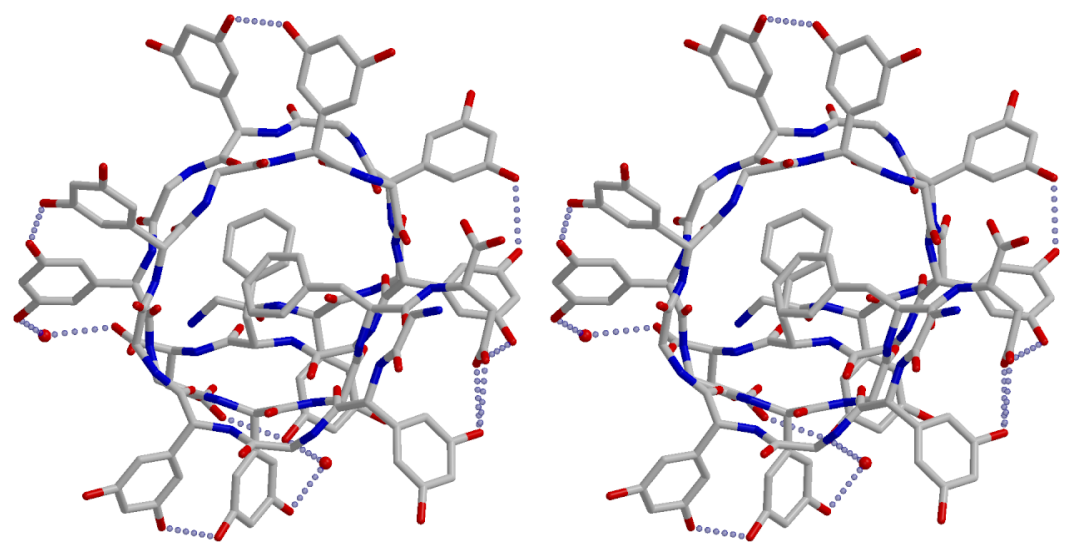

Figure 4.13

Stereo view showing the outer hydrogen-bonding shell of the feglymycin dimer.

Sidechains not participating in these interactions have been truncated for clarity. 
among the independent dimers. These include the absence of equivalents to water molecules, and break chain (ii) into two sub-chains and terminate chain (iii). The hydroxyphenyl moiety of Mpg1 in certain cases coils towards the backbone and participates in hydrogen bonds $\mathrm{O}^{4}(\mathrm{Mpg} 1-\mathrm{A})-\mathrm{N}(\mathrm{Mpg} 7-\mathrm{A})$ and $\mathrm{O}^{4}(\mathrm{Mpg} 1-\mathrm{A})-$ $\mathrm{O}(\mathrm{Mpg} 7-\mathrm{A})$.

Apart from the hydrogen bonds, hydrophobic interactions also take part in stabilising the structure. Straight after the inner hydrogen-bonding sphere there is a thick shell filled with aromatic residues that all run approximately parallel to each other and compose a $30^{\circ}$-angle with the helix axis. In addition, the benzene ring of Phe12-A stacks on the one of Phe12-B. This interaction is within the helix (and thus inside the inner hydrogen-bonding shell) and therefore the least sensitive to environmental impacts.

\section{Helical structure}

The feglymycin helix belongs to the antiparallel double-stranded double $\beta$-helix family (also known as $\pi \pi$-helix). Peptides consisting of alternating D,L-amino acids are known to form such helices. An early work studying possible conformations of poly(D-L)-peptides (Colonna-Cesari et al., 1977) postulated the existence of several families based on the helical turn, which is then dependent on the "offset" in residues participating in the "secondary"-type hydrogen bonds, i.e. the length of the sequence that is not overlapped by helix formation (Figure 4.12). Based on theoretical considerations, they concluded that the first member of this series has a minimum of 5.6 residues/turn, followed by the next member with 7.2 residues, and then 9.0, 10.7 and 12.6 residues/turn. The helix is stabilised as the pore grows since the number of close contacts with opposite residues decreases, but for a finite size helix the number of hydrogen bonds resulting from helix formation also decreases and the optimal turn is somewhere near 9.0. These molecules can also form parallel helices, but these become unstable when the helix diameter increases, the widest pore occurs at around 7.2 residues/turn.

Gramicidin was shown to form two types of these helices: one of them is an antiparallel $\beta^{5.6}$-helix (uncomplexed), while the other one is an antiparallel $\beta^{7.2}$-helix $\left(\mathrm{Cs}^{+}\right.$-complex). The first one has a left-handed screw, while the other one coils to the right. The average diameter of the $\beta^{5.6}$-helix is about $5.5 \AA$, which allows passage of ions with ion radius not greater than $1 \AA$. On the other hand, the $\beta^{7.2}$-helix has a helix diameter around $8 \AA$ and pore dimensions allowing particles as large as $4.5 \AA$ in diameter to cross the channel (Figure 4.9). These numbers are vital to understand the function, as gramicidin is a membrane channel 
peptide that causes the leakage of biological membranes. The exact conducting form is either the $\beta^{7.2}$-helix described above or a head-to-head single-stranded helix found by NMR investigations conducted in micelles and lipid bilayers (Lomize et al., 1992; Ketchem et al., 1996; Townsley et al., 2001). The $\beta^{5.6}$-helix is thought to be important for membrane insertion, and synthetic modifications of gramicidin that do not convert to the $\beta^{7.2}$-helix after membrane integration are poor ion-conductors. The gramicidin pore can allow $\mathrm{Cs}^{+}$-ions to pass (diameter $=3.6 \AA$ ), which also supports the assumption that it is the $\beta^{7.2}$-form and not the $\beta^{5.6}$-form that is responsible for membrane conduction.

On the other hand, feglymycin corresponds to a $\beta^{9.0}$-helix with the exact helical turn varying from 8.8 (chain B) to 9.2 (chain A) residues/turn. In this case, the channel diameter is 9.5-12 $\AA$ and the pore size is between 6.5-9.0 $\AA$. The channel is wide enough to accommodate a benzene ring since there are two phenylalanine sidechains in the pore. In both of the crystal structures, one of which crystallised from 4\% PEG8000 (virtually from water), the other one from $30 \%$ isopropanol corresponding to an apolar environment, the conformations of the peptides were found to coincide very closely. It is therefore likely that feglymycin retains the $\beta^{9.0}$-helix when inserts into the membrane (if at all) and does not readily undergo such substantial conformational rearrangement as found for gramicidin.

\section{Crystal packing}

In both of the crystal forms, feglymycin composes infinite helical chains of dimers enforced by the $6_{5}$ or the $6_{4}$ crystallographic screw axis. These chains are so symmetrical that, not regarding certain atoms, a $180^{\circ}$-rotation perpendicular to $\mathbf{c}$ transforms the helix to itself. This property may be responsible for merohedral twinning present in these crystals. Although a dimer is fairly symmetrical, the two chains (class A and B) that build it up differ in conformation at the termini, which provides directionality to the dimers. Reversing the directionality would involve $180^{\circ}$ rotations around the $C^{\alpha}-C^{\beta}$ bond for two phenylalanine sidechains. This not likely to happen spontaneously as to remove the steric hindrance would require the folding out of the helix. The side of the dimer containing the $\mathrm{N}$-terminus of the class A chain will be referred to as "head" and the other one as "tail".

\section{Crystal form I}

The asymmetric unit of crystal form I contains three dimers that build up one helical chain and all three theoretically possible fittings, namely head-to-head, head-to-tail and tailto-tail are present amongst the molecules. One of the dimers has arbitrarily been chosen as 
dimer I, and the other two dimers are numbered sequentially as they follow each other in the helix. In the apparent higher symmetry space group ( (6 $\left.5_{5} 22\right)$ dimer I would be a symmetry equivalent of dimer III and dimer II-chain A of dimer II-chain B.

Dimer I is connected to a symmetry equivalent of dimer III via a head-to-head interaction. Hydrogen bonds between mainchain amide and carbonyl atoms, namely N(Mpg1A/I)-O(Mpg5-A/III'), O(Mpg1-A/I)-N(Mpg5-A/III')，N(Val3-A/I)-O(Val3-A/III')，O(Val3A/I)-N(Val3-A/III'), N(Mpg5-A/I)-O(Mpg1-A/III') and O(Mpg5-A/I)-N(Mpg1-A/III') constitute the dimer interface, which is nearly a perfect continuation of the helix-forming hydrogen bonds. Hydrophobic interactions between the aromatic rings of Dpg2-A/I-Dpg4A/III' and Dpg4-A/I-Dpg2-A/III' provide further strength. At the other end, dimer I is linked to dimer II by a tail-to-tail interface that consists of hydrogen bonds N(Mpg1-B/I)-O(Val3B/II), O(Mpg1-B/I)-N(Val3-B/II), O4(Mpg1-B/I)-O(Mpg5-B/II), N(Val3-B/I)-O(Mpg1$\mathrm{B} / \mathrm{II}), \quad \mathrm{O}(\mathrm{Val} 3-\mathrm{B} / \mathrm{I})-\mathrm{N}(\mathrm{Mpg} 1-\mathrm{B} / \mathrm{II})$ and $\mathrm{O}(\mathrm{Mpg} 5-\mathrm{B} / \mathrm{I})-\mathrm{O} 4(\mathrm{Mpg} 1-\mathrm{B} / \mathrm{II})$, and aromatic interactions between sidechains at Mpg1-B/I-Mpg1-B/II and Dpg2-B/I-Dpg2-B/II. The mainchain atoms do not participate as much as observed at the head-to-head interface but the N-terminal hydroxyphenyl moiety also takes part in the interaction. To complete the palette, there is a head-to-tail interaction between dimer II and III, constituted by hydrogen bonds $\quad \mathrm{N}(\mathrm{Mpg} 1-\mathrm{A} / \mathrm{II})-\mathrm{O}(\mathrm{Mpg} 3-\mathrm{A} / \mathrm{III}), \quad \mathrm{O}(\mathrm{Mpg} 1-\mathrm{A} / \mathrm{II})-\mathrm{N}(\mathrm{Mpg} 3-\mathrm{A} / \mathrm{III}), \quad \mathrm{O}^{4}(\mathrm{Mpg}-\mathrm{A} / \mathrm{II})-$ $\mathrm{O}(\mathrm{Mpg} 5-\mathrm{A} / \mathrm{III}), \mathrm{O}(\mathrm{Val} 3-\mathrm{A} / \mathrm{II})-\mathrm{N}(\mathrm{Mpg} 1-\mathrm{A} / \mathrm{III})$ and N(Val3-A/II)-O(Mpg1-A/III), and the aromatic stacking between Dpg2-A/II-Dpg2-B/III. This interface is similar to the tail-to-tail interaction except for the participation of the hydroxylphenyl group. The extensive involvement and the diversity of interfaces between the dimers account for the conformational variety of Mpg1. As this residue does not follow the chirality alternation, it is not held tight and flexible enough to adapt to the environment.

It is instructive to compare the different interfaces regarding the empty space they enclose. In the head-to-head interface the terminal Mpg residues are displaced towards the wall of the helix by a hydrogen bond, and as a result, they open up and give access to the hollow space inside the feglymycin dimer, which is big enough to accommodate an 8-residuelong polyethyleneglycol chain (Figure 4.14a). In contrast, the hydrophobic interaction between these amino acids observed in the tail-to-tail dimer attracts them towards the helix centre and blocks the entry to the dimer interior (Figure 4.14b). The head-to-tail interface occupies a position in between. 


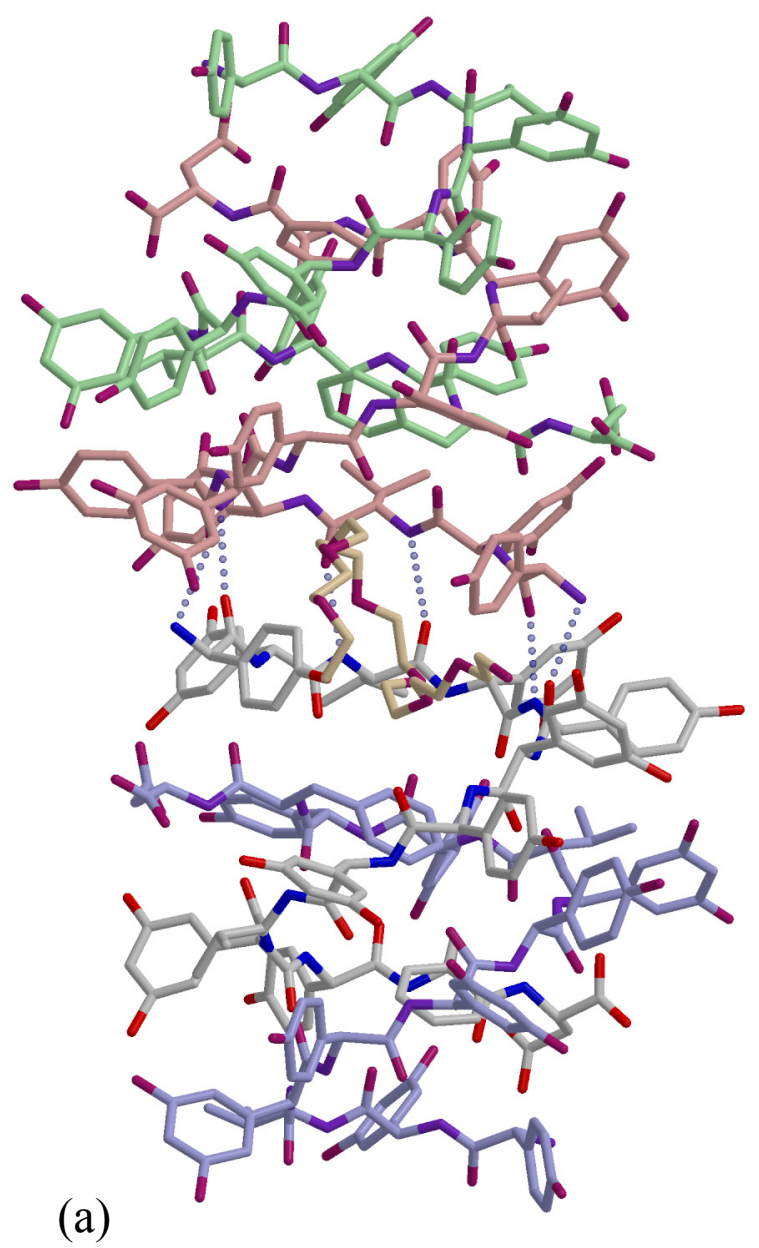

(b)

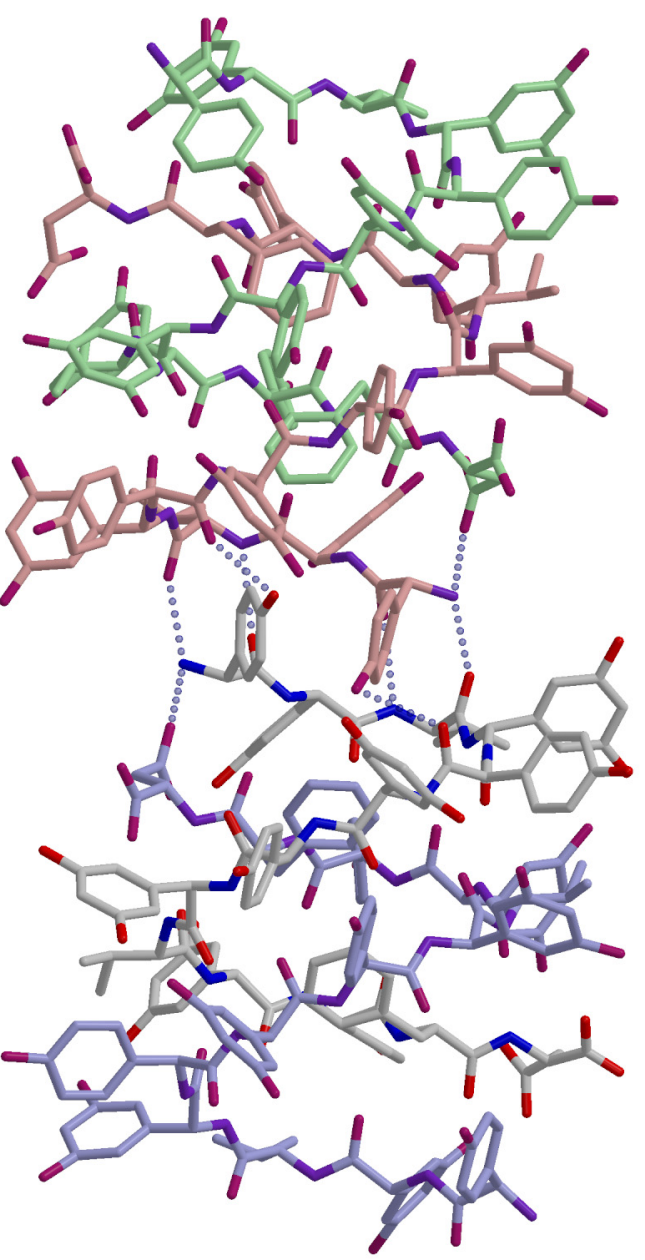

Figure 4.14

Interdimer contacts in the feglymycin crystal. The tetramer shown consists of two dimers (grey-blue and redbrown-green). (a) The head-to-head interface, where the phenyl rings of terminal Mpg residues open up and give access to the molecular interior. The cavity is big enough to accommodate a polyethyleneglycol chain (light brown). (b) The tail-to-tail interface, in which these phenyl rings interact with each other strongly and close the entry to the internal hollow.

On the other hand, very few interactions can be observed between the helices that build up the crystal. These are limited to some hydrogen bonds between $\mathrm{O}^{5}(\mathrm{Dpg} 2-\mathrm{A} / \mathrm{I})-\mathrm{O}^{5}(\mathrm{Dpg} 4-$ A/III'), O ${ }^{5}(\mathrm{Dpg} 2-\mathrm{B} / \mathrm{I})-\mathrm{O}^{5}\left(\mathrm{Dpg} 2-\mathrm{A} / \mathrm{II}^{\prime \prime}\right), \quad \mathrm{O}^{5}(\mathrm{Dpg} 2-\mathrm{B} / \mathrm{I})-\mathrm{O}^{5}(\mathrm{Dpg} 2-\mathrm{B} / \mathrm{III} "), \quad \mathrm{O}^{5}(\mathrm{Dpg} 2-\mathrm{B} / \mathrm{II})-$ $\mathrm{O}^{5}\left(\mathrm{Dpg} 2-\mathrm{B} / \mathrm{III} \mathrm{I}^{\prime}\right), \mathrm{O}^{5}(\mathrm{Dpg} 2-\mathrm{B} / \mathrm{II})-\mathrm{O}^{5}(\mathrm{Dpg} 2-\mathrm{A} / \mathrm{II} ")$ and $\mathrm{O}^{5}(\mathrm{Dpg} 4-\mathrm{B} / \mathrm{I})-\mathrm{O}^{5}(\mathrm{Dpg} 4-\mathrm{B} / \mathrm{III} " ')$. There are also big holes in the crystal filled with disordered water, and in other regions the helices are concentrated. 


\section{Crystal form II}

This crystal form contains four dimers that build up two helices generated by the 64 axis. The two helices are not related by crystallographic symmetry, but closely resemble each other. One helix is built up by dimer I and II, while the other one by dimer III and IV in a nearly identical fashion. There is no direct interaction between the crystallographically independent helices, only among symmetry-related ones. This is likely to be the reason for the fragility of this crystal form compared to the other one. The twofold axis introduced by twinning holds for one helix, but not for the second, and introduces an apparent disorder (Figure 4.6). As the two helices are almost identical, only one of them is presented.

The two independent dimers in the helix associate first to a tetramer via a tail-to-tail interaction nearly identical to that observed in crystal form I. The only difference is the appearance of an additional hydrogen bond between $\mathrm{O}^{4}(\mathrm{Mpg} 1-\mathrm{B} / \mathrm{I})-\mathrm{O}^{4}(\mathrm{Mpg} 1-\mathrm{B} / \mathrm{II})$. These tetramers then associate via head-to-head interfaces as described above. The helices interact with symmetry related ones via four hydrogen bonds between $\mathrm{O}^{5}(\mathrm{Dpg} 2-\mathrm{B} / \mathrm{I})-\mathrm{O}^{5}\left(\mathrm{Dpg} 2-\mathrm{B} / \mathrm{I}^{\prime}\right)$, $\mathrm{O}^{5}(\mathrm{Dpg} 2-\mathrm{B} / \mathrm{I})-\mathrm{O}^{5}(\mathrm{Dpg} 2-\mathrm{B} / \mathrm{II}), \quad \mathrm{O}^{5}(\mathrm{Dpg} 2-\mathrm{B} / \mathrm{II})-\mathrm{O}^{5}\left(\mathrm{Dpg} 2-\mathrm{B} / \mathrm{II} \mathrm{I}^{\prime}\right), \quad \mathrm{O}^{5}(\mathrm{Dpg} 2-\mathrm{B} / \mathrm{II})-\mathrm{O}^{5}(\mathrm{Dpg} 2-$ $\left.\mathrm{B} / \mathrm{I}^{\prime}\right)$, which are also analogous to interactions in crystal form I. Comparison of the interfaces in terms of empty space they surround yields the same result with the exception that in this crystal form the head-to-head cavity is occupied by two isopropanol molecules.

\section{BIOLOGICAL IMPLICATIONS}

Feglymycin is a unique antibiotic in terms of sequence and antimicrobial spectrum. The structure determination revealed that it closely resembled the membrane ion channel peptide gramicidin. Feglymycin forms dimers in aqueous and also in predominantly apolar environments, and the dimer is very likely to be the biologically active unit. Under favourable conditions the dimers can associate to tetramers, but it needs still to be determined whether this is relevant to the function.

\section{Channel formation}

The width of biological membranes varies among the species, but it has been shown that a minimum size of about $30 \AA$ is necessary to span bacterial membranes. This value is only approximate and compounds lying slightly under this limit have also been shown to form ion channels under certain conditions (Duclohier et al., 1998). The gramicidin dimer in the 
$\mathrm{Cs}^{+}$-complex, which is believed to be relevant to the membrane conducting form, is about $25 \AA$ in length if one considers the $\mathrm{C}^{\alpha}$-atoms only or $28 \AA$ if all the atoms are regarded and is long enough to span through the membrane. Other physico-chemical properties like hydrophobic surfaces essential for membrane integration and a hole formed by the structure that allows passage of ions smaller than a certain size also support this assumption.

In contrast, feglymycin has a shorter sequence and a wider hole; therefore the dimer is only $18 \AA$ long measuring between the farthest $C^{\alpha}$-atoms or $20 \AA$ when all atoms are included. This is significantly less than required to span a biological membrane (Figure 4.8). Moreover, the channel in the feglymycin dimer is blocked by four amino acid sidechains (Figure 4.9). Two of them, namely the phenyl rings of Mpg1-A and Mpg1-B are shown to possess the necessary flexibility to open and close, but even minor displacements of the Phe12-A and Phe12-B sidechains are not feasible because of extensive steric hindrance.

\section{Membrane processes}

It is likely that feglymycin can easily integrate into the membrane as it has hydrophobic residues on its surface, and might function as an ion carrier rather than a channel between the two sides. There are ion carriers known that have alternating D,L-amino acids in their sequence, like valinomycin (Duax et al., 1996). The transport is usually highly selective for a given anion or a cation, which is $\mathrm{K}^{+}$in the case of valinomycin (Tosteson et al., 1967). However, such selectivity is not expected for feglymycin, where the internal cavity can accommodate nearly any particles under a certain size. Nevertheless, as feglymycin can integrate into the membrane, it may also be able to penetrate it, and interact with enzymes in the cytosol. This mechanism may be important in the HIV-inhibitory activity and suggests a scheme for the transport of feglymycin into the infected cell.

\section{Interaction surface}

Concerning its structure, feglymycin is a perfectly tailored molecule for a specific interaction. First, the structure is considerably rigid, which is a conspicuous feature of virtually all natural inhibitor molecules. Secondly, the molecular organisation of the dimer utilises all Dpg residues in stabilising the structure, while the Mpg residues are involved in these interactions to a much lesser extent. On the other hand, Mpg residues are ideal in constituting a very well defined interaction surface: rotation around the $C^{\alpha}-C^{\beta}$ bond does not affect the position of its hydroxyl, which is therefore stationary. A well-defined hydrogen- 

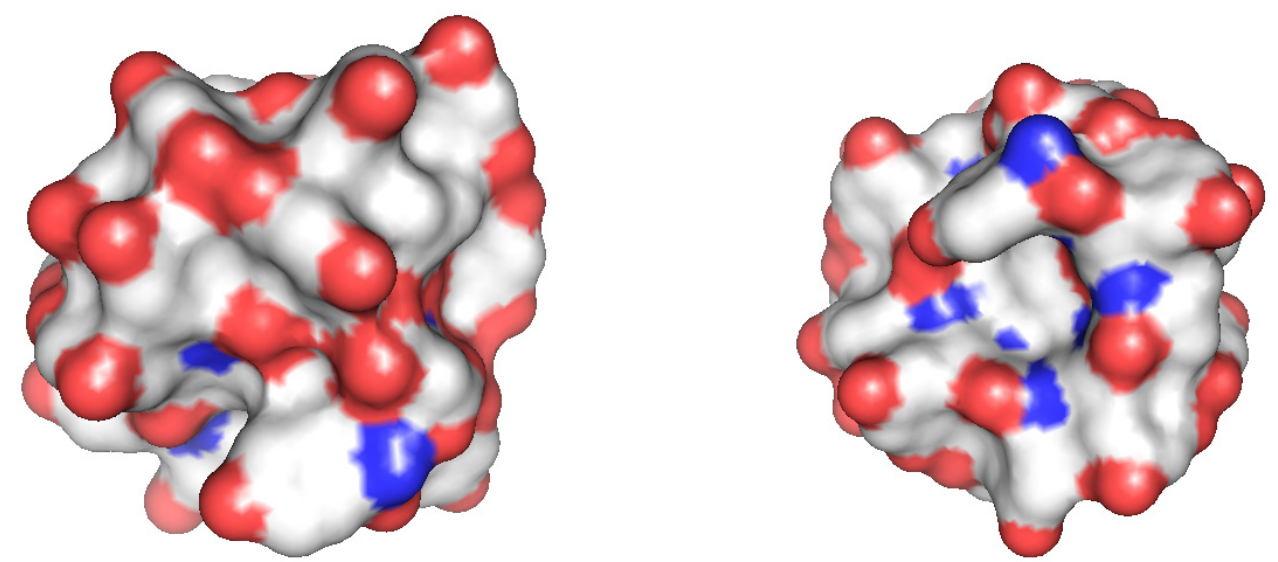

\section{Figure 4.15}

The surface of the feglymycin dimer showing potential interactions (hydrogen bonds and hydrophobic recesses). The orientation in (a) corresponds to Figure 4.8 and in (b) to that on Figure 4.9 and shows that the feglymycin channel is blocked. Carbon atoms are grey, nitrogen atoms are blue and oxygen atoms are red.

bond pattern is important in molecular recognition: hydrogen bonds provide the required specificity, even if the affinity is a result of an extensive number of non-specific, hydrophobic interactions. Moreover, hydrophobic patches are also present on the feglymycin surface: the valine residues constitute recesses on the molecular exterior at certain places that might be important in connecting feglymycin to its target (Figure 4.15). As feglymycin retains its conformation in both polar and apolar environments, it is also conceivable that it exerts inhibitory activity within the membrane, i.e. on the membrane-embedded surface of a membrane-bound or transmembrane protein. In the light of the foregoing evidence it seems therefore likely that feglymycin is not a membrane agent, but a strong enzyme inhibitor acting on a wide range of targets. 



\section{CRYSTAl StRucture OF LIPOPEPTIDE ANTIBIOTICS}

\section{INTRODUCTION}

Lipopeptide antibiotics constitute a class of antibacterial agents that are highly active against multiresistant bacteria. Amphomycin, the first member of the series, was discovered more than 50 years ago (Heinemann et al., 1953), and was followed by the isolation of crystallomycin (Lomakina \& Brazhnikova, 1959), aspartocin (Shay et al., 1960), glumamycin (Shibata et al., 1962), laspartomycin (Naganawa et al., 1968), tsushimycin (Shoji et al., 1968; Shoji \& Otsuka, 1969) and other antibiotics belonging to the same group. However, probably because of their hemolytic properties and resulting toxicity, interest in this antibiotic class has waned, although the antibiotic daptomycin discovered by Eli Lilly progressed in the 1980s to clinical phase I and phase II trials. As reversible skeletal muscle toxicity was observed at high doses (Tally et al., 1999) and treatment failures were experienced in patients with Staphylococcus aureus endocarditis (Garrison et al., 1989), the development was suspended. More recently, the increased occurrence of infections caused by resistant Gram-positive bacteria led to renewed interest in daptomycin and in 1997 Cubist Pharmaceuticals licensed the drug from Lilly and reinitiated clinical trials. Daptomycin is currently being approved as an intravenous formulation (under the trade name Cidecin) in the USA, and introduction to the European market is intended in the near future (Rotschafer et al., 1988; Bronson \& Barrett, 2001; Strahilevitz \& Rubinstein, 2002; Linden, 2002; Woodford, 2003; Abbanat et al., 2003).

The chemical structure of amphomycins was first investigated in the 1960s. Performing chemical degradation studies on the antibiotic glumamycin, Inoue and Fujino (Inoue, 1962a; Inoue, 1962b; Inoue, 1962c; Fujino, 1965) determined the amino acid composition and the structure of the fatty acid component and proposed two possible sequences for the peptide core. Investigating the antibiotic amphomycin, Bodanszky et al. (1973) arrived at an alternative sequence, and revised the one reported for glumamycin by proving the identity of the two antibiotics. More recently, Vértesy et al. (2000) deduced the chemical structure of the related friulimicins by two independent spectroscopical methods and confirmed the correctness of one of the sequences suggested by Fujino (1965). According to this, amphomycin antibiotics possess a peptide framework consisting of a ten-membered 


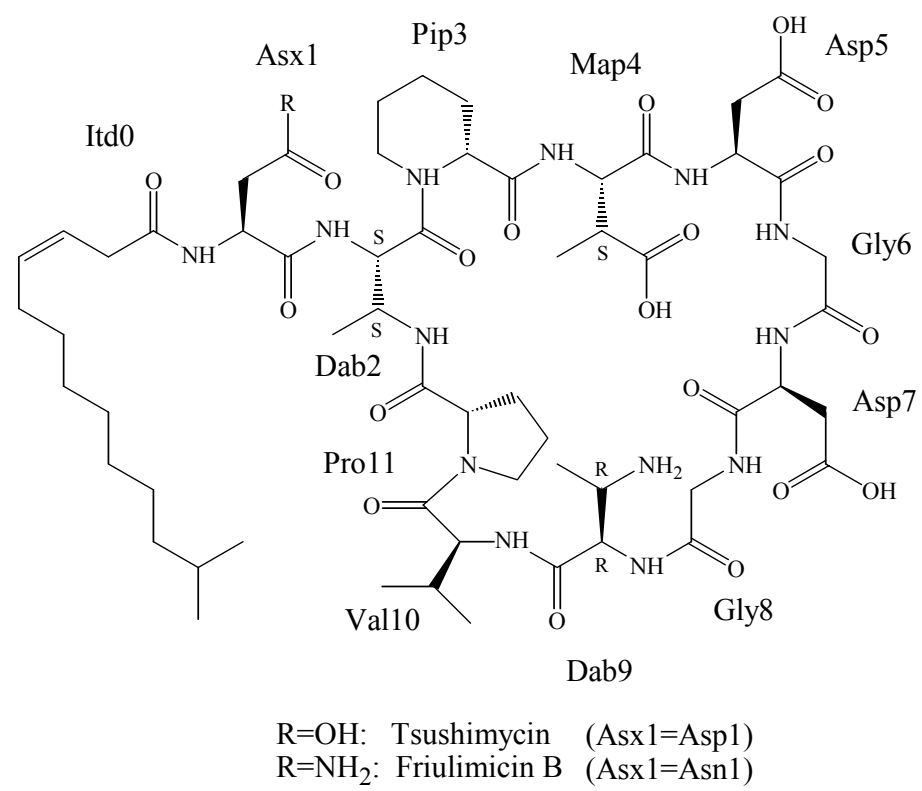

\section{Figure 5.1}

Chemical structure of tsushimycin and friulimicin B according to Vértesy et al. (2000).

cyclopeptide ring and an exocyclic amino acid, the N-terminus of which is acylated by a fatty acid residue (Figure 5.1). Differences among the group members arise principally in the structure of the fatty acid substituent. On the other hand, daptomycin (Figure 5.2) was shown to have a markedly different structure that shows homology to the general amphomycin sequence only in consisting a cyclic decapeptide core, which has aspartates at positions 4 and 6 relative to the attachment point of exocyclic amino acids, and the presence of a fatty acid residue (Debono et al., 1988).

Amphomycin was found to be active against Gram-positive bacteria, but the exact mechanism of action has not been conclusively determined. Early studies indicated that amphomycin was a specific inhibitor of bacterial cell-wall synthesis, acting at the level of phospho-N-acetylmuramoyl-pentapeptide-transferase (MraY), which catalyses the transfer of the UDP-MurNAc-pentapeptide to the undecaprenyl carrier (Tanaka et al., 1977; Tanaka et al., 1979; Tanaka et al., 1982). It was also found that amphomycin inhibited the synthesis of dolichol-linked saccharides in eukaryotes, which is essential in the glycosylation of glycoproteins that contain mannose and GlcNAc, although higher concentrations were necessary to achieve an effect similar to that on the bacterial enzyme (Kang et al., 1978). It was proposed that amphomycin acts by binding the substrate dolichylmonophosphate with high affinity and in a $\mathrm{Ca}^{2+}$-dependent manner (Banerjee, 1989), as no binding was observed in the absence of $\mathrm{Ca}^{2+}$. Since dolichylphosphate is similar in structure to the undecaprenyl 


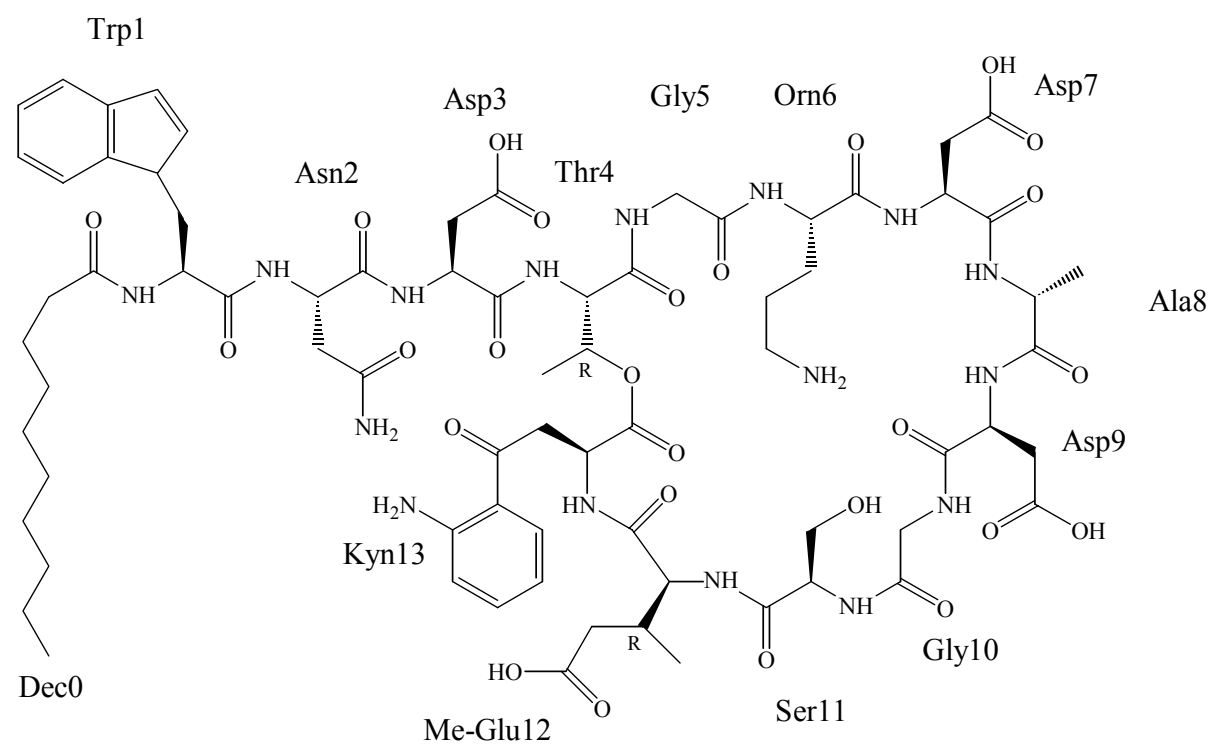

\section{Figure 5.2}

Chemical structure of daptomycin.

carrier in prokaryotes, it may be concluded that bactericidal action of amphomycin is achieved by complexing this compound and thereby preventing murein biosynthesis. The amphomycin-relative daptomycin was first reported to act by inhibiting peptidoglycan biosynthesis at the stage of formation of the nucleotide-linked sugar-peptide precursors (Allen et al., 1987). However, it was pointed out by Canepari et al. (1990) that daptomycin does not enter the cell and suggested the synthesis of lipoteichoic acids occurring in the cytoplasmic membrane as the site of action. Teichoic acids (Figure 5.3) are present on the outer surface of Gram-positive bacteria and are involved in the circulation of divalent cations (Lambert et al., 1977). Lilly scientists published results presenting evidence that daptomycin dissipates the membrane potential without affecting the chemical gradient across the membrane and proposed this as being the basis of antimicrobial action (Alborn et al., 1991).

It is instructive to compare lipopeptides with polymyxin antibiotics. Similarly to lipopeptides, polymyxins are also cyclopeptide antibiotics, but consist of a seven-membered peptide ring, exocyclic amino acids and a fatty acid tail (Vogler et al., 1965). In contrast to lipopetides, polymyxins are polycationic species as most of the amino acids have amino groups on their sidechains. Polymyxins therefore readily bind anionic species like phosphatidylglycerols that results in a rapid permeability change of the cell membrane, release of cellular materials and subsequent cell death (El Mashak \& Tocanne, 1980; Yin et al., 2003). 


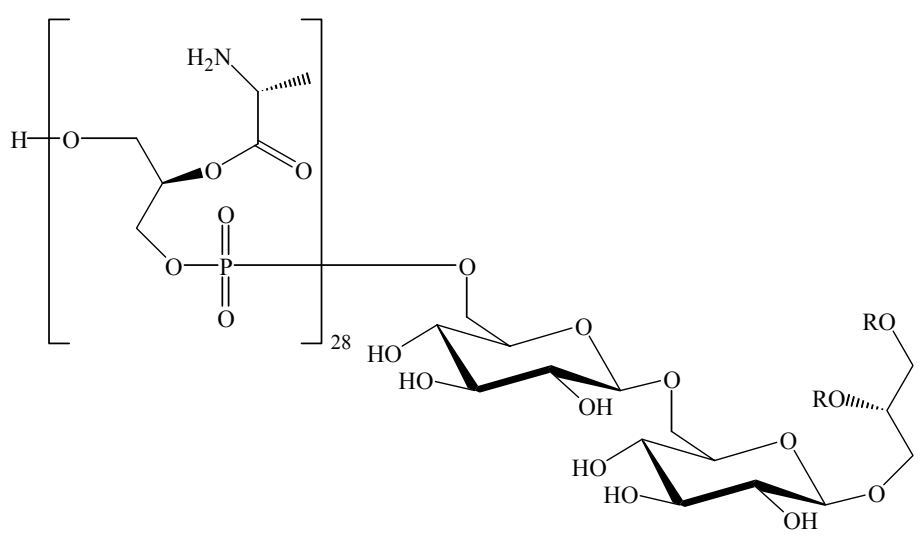

Figure 5.3

Structure of lipoteichoic acid of Staphylococcus aureus. R represents a long alkyl chain.

Lipopetide antibiotics possess superior antibacterial potency, as they are rapidly bactericidal not only against multiresistant Staphylococcus aureus, but also against Enterococcus spp, a property that none of the currently licensed antibiotics can claim if administered alone. Resistance to lipopetide antibiotics is rare; daptomycin, the only member being evaluated in clinical trials is active against $99 \%$ of tested clinical isolates; in addition, the distribution of minimum inhibitory concentration values were unimodal, and therefore the existence of a defined resistance mechanism is unlikely (Barry et al., 2001). The level of $\mathrm{Ca}^{2+}$ ions in the medium proved to be important when tested against less susceptible isolates, as in trials on $\mathrm{Ca}^{2+}$-supplemented media corresponding to physiological $\mathrm{Ca}^{2+}$-levels minimum inhibitory concentration values were found to be two- to fourfold lower. Lipopeptides have limited stability under physiological conditions; spontaneous chemical reactions readily occur because of the presence of the Asp-Gly sequence pattern, which occurs more than once in certain members, and results in the loss of activity (Geiger \& Clarke, 1987). This property provides a mechanism for self-clearance from the environment and may contribute to preventing the development of resistance. These antibiotics have not been employed as food additives in animal husbandry, so resistance could not have developed in enterococci and spread in the society. Given the superior antibacterial activity primarily against problematic microbes and inherent ways for keeping development of antibacterial resistance at a low level, lipopeptides are perfect antibiotics for reinforcing or replacing glycopeptides as a last line of defence against deadly bacterial infections. 


\section{STRUCTURE DETERMINATION}

\section{Crystallisation}

Three different samples belonging to the group of lipopeptide antibiotics were kindly provided by Aventis Pharma Deutschland in development-scale quantities: A-1437 B (probably identical with tsushimycin, 93\% pure) and A-1437 D (friulimicin) as $\mathrm{Na}^{+}$-salt ( $85 \%$ pure) and as $\mathrm{Ca}_{2} \mathrm{Cl}_{2}$-salt $(93 \%$ pure). The slight impurity is a consequence of isomerisation reactions at aspartate residues and since it proved excessively cost-intensive, no further purification was attempted.

Lipopetide antibiotics are known to form crystals, and in the purification protocol of friulimicin crystallisation was included as the final step. It was shown that only the active antibiotic crystallises under the established conditions and thereby can be separated from the isomerised, inactive substance. As starting material, the $\mathrm{Na}^{+}$-salt of friulimicin is used, which is dissolved in water, $\mathrm{pH}$-adjusted to 6.4 and converted to the $\mathrm{Ca}^{2+}$-form by the addition of $\mathrm{CaCl}_{2}$. Crystallisation is initiated by slowly increasing the ethanol content of the solution.

As sufficient amounts from all samples were available, a slightly modified procedure was tried in a batch-crystallisation setup. Since slow addition requires adequate instrumentation, which was not available, $250 \mu \mathrm{l}$ of an aqueous solution containing $40 \mathrm{mg} / \mathrm{ml}$ $\mathrm{Na}^{+}$-friulimicin was simply mixed with an equal volume of the crystallisation condition in reservoirs of tissue culture plates. The crystallisation condition consisted of $0.1 \mathrm{M} \mathrm{Na}$ cacodylate/cacodylic acid $\mathrm{pH}=6.0$ or $0.1 \mathrm{M} \mathrm{NaAc} / \mathrm{HAc} \mathrm{pH}=5.4,0.2 \mathrm{M} \mathrm{CaCl}_{2}$ and $30-50 \%$ acetonitrile or ethanol. Large needle crystals appeared within a day. However, picking crystals out of the reservoir proved complicated and quality of the crystals was not sufficient and therefore this approach was discontinued.

As a method of choice, the hanging drop method was selected, and the foregoing procedure was modified accordingly. Unfortunately, no improvement in crystal quality was observed and for this reason, screening was performed with all available kits (Hampton Crystal Screen I, Crystal Screen II, JBScreen 7, 8 and 9). Crystals were obtained from nearly all conditions that contained $\mathrm{CaCl}_{2}$ and an organic solvent. The most promising crystals were grown from $0.1 \mathrm{M} \mathrm{NaAc} / \mathrm{HAc} \mathrm{pH}=4.6,0.2 \mathrm{M} \mathrm{CaCl}_{2}$ and $30 \%$ isopropanol. It is noteworthy that according to crystallisation experiments performed at Aventis Pharma, isopropanol proved not suitable as an organic solvent since it led to amorphous precipitation. However, replacement of isopropanol in the foregoing condition with the recommended ethanol or 
acetonitrile yielded no crystals. Since it was of higher purity and calcium was essential for crystallisation, the $\mathrm{Ca}_{2} \mathrm{Cl}_{2}$-form was used in further experiments. After optimisation, wellformed block crystals were obtained from $0.1 \mathrm{M} \mathrm{NaAc} / \mathrm{HAc} \mathrm{pH}=4.6,0.2 \mathrm{M} \mathrm{CaCl}$, $30 \%$ isopropanol and $10 \%$ glycerol that diffracted to 3-3.5 $\AA$ resolution on a rotating anode (crystal form I, Figure 5.4).

The crystals were not very stable at room temperature and began to decompose after one week. This observation hinted that the stability of the substance is not sufficient at room temperature and diffraction limit may be improved by performing crystallisation experiments in the cold room. As crystallisation conditions vary with the temperature, first a coarse grid screen was performed and new conditions were then optimised. Block crystals having a different habit than those obtained at room temperature grew in the course of several days from $0.1 \mathrm{M} \mathrm{NaAc} / \mathrm{HAc} \mathrm{pH}=4.6,0.14 \mathrm{M} \mathrm{CaCl}_{2}$ and 14\% isopropanol. These crystals diffracted much better (1.8 $\AA$ on a rotating anode, atomic resolution at BESSY), but proved to be of another crystal modification and were non-merohedrally twinned (crystal form II, Figure 5.4).

To promote the growth of crystals belonging to crystal form I, a step-wise cooling procedure was selected. A coarse grid-screen was prepared and the temperature was held constant at $12{ }^{\circ} \mathrm{C}$ in a Hampton incubator. Crystals resembling in shape the ones grown at room temperature were obtained from $0.1 \mathrm{M} \mathrm{NaAc} / \mathrm{HAc} \mathrm{pH}=4.6,30 \%$ isopropanol, $10 \%$ glycerol and $0.10 \mathrm{M} \mathrm{CaCl}_{2}$. Based on this finding a modified coarse screen followed by optimisation was performed at $4{ }^{\circ} \mathrm{C}$. Crystals identical in morphology to that of crystal form I were obtained from $0.1 \mathrm{M} \mathrm{NaAc} / \mathrm{HAc} \mathrm{pH}=4.6,38 \%$ isopropanol, and $0.06 \mathrm{M} \mathrm{CaCl}_{2}$ within a week. It must be noted that crystal formation was extensively sensitive to $\mathrm{Ca}^{2+}$-concentration, as no crystals grew outside the range of $0.05-0.07 \mathrm{M} \mathrm{CaCl}_{2}$. These crystals were very fragile and to avoid mechanical stress upon freezing, crystallisation conditions were modified to

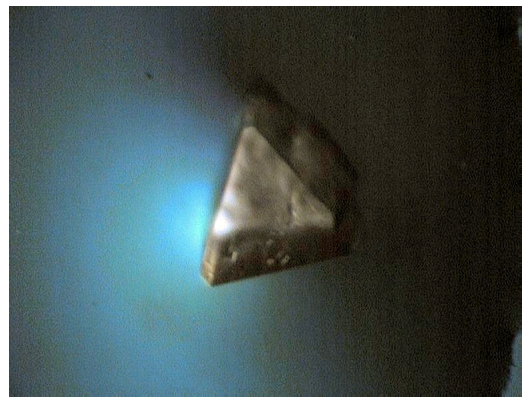

Friulimicin crystal form I

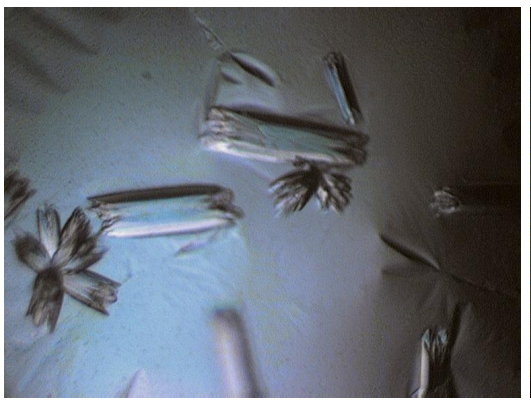

Friulimicin crystal form II

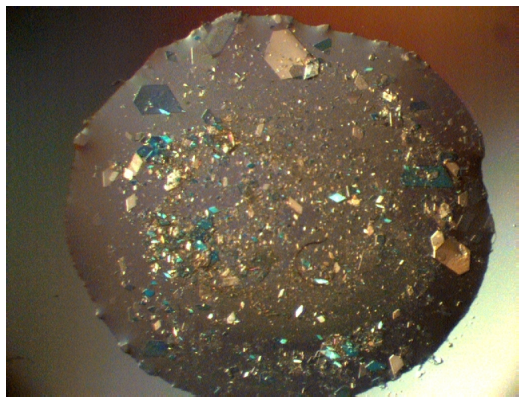

Tsushimycin

Figure 5.4

Crystal morphologies of friulimicin and tsushimycin. 
include the cryoprotectant to $0.1 \mathrm{M} \mathrm{NaAc} / \mathrm{HAc} \mathrm{pH}=4.6,32 \%$ isopropanol, $0.30 \mathrm{M}$ sucrose and $0.06 \mathrm{M} \mathrm{CaCl}_{2}$. These crystals indeed belonged to crystal form $\mathbf{I}$ and diffracted to $2.5 \AA$ in-house and to $1.6 \AA$ at BESSY.

Examination of the crystallisation procedure received from Aventis Pharma revealed one more possibility for the improvement of crystal quality. It was found that the decomposition rate depends on the $\mathrm{pH}$, being the fastest at the isoelectric point $(\mathrm{pH}=4.4)$ and decreasing steeply towards neutral $\mathrm{pH}$. As the crystallisation was not excessively sensitive to alteration of the $\mathrm{pH}$, the buffer was changed and comparable quality crystals were grown from Na-cacodylate/cacodylic acid $\mathrm{pH}=6.0,40 \%$ isopropanol and $0.08 \mathrm{M} \mathrm{CaCl}_{2}$. Unfortunately, no significant improvement in diffraction limit was observed.

Independently of friulimicin, crystallisation experiments were started with tsushimycin as well. Although the difference between friulimicin and tsushimycin are marginal, there is a considerable difference in their toxicity and antimicrobial potency. It was also observed when working with peptaibols that one-atom modifications can substantially alter crystallisation properties and therefore tsushimycin crystals might be the means of overcoming the difficulties posed by friulimicin. As no crystals were obtained from any of the conditions used for friulimicin, the procedure from Aventis Pharma was used as a starting point. Small crystals were obtained from $0.1 \mathrm{M} \mathrm{NaAc} / \mathrm{HAc} \mathrm{pH}=4.6,30 \%$ ethanol and $0.4 \mathrm{M} \mathrm{CaCl}_{2}$. It was recognised early that crystallisation demanded a lower $\mathrm{pH}$, since the isoelectric point of tsushimycin is at about $\mathrm{pH}=3.6$, where acetate has little buffer capacity. To retain reproducibility, the $\mathrm{pH}$ was set by mixing $1.0 \mathrm{M} \mathrm{NaAc} / \mathrm{HAc} \mathrm{pH}=4.0$ with different amounts of $1.0 \mathrm{M}$ HAc. Thin plate-like crystals were grown from $0.1 \mathrm{M} \mathrm{NaAc} / \mathrm{HAc} \mathrm{pH}=4.0$, $0.12 \mathrm{M} \mathrm{HAc}, 38 \%$ ethanol and $0.80 \mathrm{M} \mathrm{CaCl}_{2}$ (Figure 5.4). These crystals were grown at room temperature and were sufficiently stable, starting to decompose after 6 months. To support cryoprotection, later trials were supplemented with 0.40 M 1,6-hexanediol.

\section{Measurement}

\section{Friulimicin crystal form I}

Since crystals were grown in the cold room and sucrose had been included in the condition provided sufficient cryoprotection, a suitable crystal was selected, taken out with an adequately sized loop and dipped into a Dewar vessel full of liquid nitrogen. The crystal was characterised by collecting datasets on a Bruker rotating anode, Osmic focusing mirrors and Bruker SMART6000 4K CCD detector. Diffraction was observed to $2.5 \AA$ resolution. 
However, problems arose in unit cell determination as different indexing algorithms implemented in Proteum gave different answers that all fitted the crystal lattice within experimental error. Difference vector-based algorithms yielded a primitive trigonal cell with cell dimensions $\mathrm{a}=\mathrm{b}=73.4 \AA$ and $\mathrm{c}=105.7 \AA$, while the Fourier-algorithm gave a rhomohedral cell with $\mathrm{a}=\mathrm{b}=\mathrm{c}=63.2 \AA$ and $\alpha=\beta=\gamma=71^{\circ}$ (corresponding to $\mathrm{a}=\mathrm{b}=73.6 \AA$ and $\mathrm{c}=140.1 \AA$ in hexagonal setting). It turned out later that the crystal had an unusual intensity distribution and strong reflections constituted a sub-lattice; this property cause algorithms employing Fouriertransformation to give the wrong answer. However, the program cell_now indexes the lattice routinely and selects automatically the right primitive trigonal cell.

After the ambiguity with the unit cell had been resolved, datasets were collected in order to extract the anomalous signal originating from the incorporated $\mathrm{Ca}^{2+}$-ions. Three $\varphi$-scans and five $\omega$-scans were performed, which provided two approximately equivalent datasets in terms of data redundancy and precision. Integration was performed using SAINT and scaling with SADABS. The correlation coefficient between the signed anomalous differences calculated independently from the two types of scans was calculated and then all runs were scaled together in a subsequent SADABS run and anomalous differences were derived from the complete dataset with XPREP (Bruker Nonius, 2002).

Not regarding high apparent mosaicity, crystal quality seemed satisfactory and a strong anomalous signal was expected, but it was indicated by the statistics that the signal extends to only $4.0 \AA$ resolution. It was realised at this point that the unusual intensity distribution $\left(\left|E^{2}-1\right|=1.215\right)$ might be responsible for this result: with more than $50 \%$ of the reflections below $2 \sigma$, the crystal behaves like a centred lattice but as the pseudo-"systematically absent" reflections do not follow a regular pattern, they cannot be filtered out. This has two consequences on data processing and statistics:

(a) Reflection profiles. In SAINT, all reflections contribute to the determination of standard reflection shapes, which in this case is suffering from the overwhelming number of "missing" reflections. A solution might be the exclusion of weak reflections from this procedure (or not to use profile fitting at all, e. g. as in the program EVALCCD) or the determination of a real-space sub-lattice, which can be used to solve the phase problem and then convert the results to the original cell. This latter solution is based on the assumption that pure translational symmetry is present in the unit cell, and selecting a sub-cell would only convert the non-crystallographic symmetry into crystallographic one, and intensity distribution would then follow 
regular Wilson-statistics. When a solution is achieved, it can be extended to the original cell and thus overcome the phase problem.

(b) Distorted statistics. As there is a high number of reflections with low intensity, anomalous differences calculated from them are also weak and unreliable. As the programs print an average value for each resolution shell, this value is artificially low, since it includes all reflections, half of which do not contribute to the intensity and only increase the standard deviation. However, the weak reflections are not used in anomalous substructure solution, since direct method programs select only the strongest $10 \%$ of the data, therefore a resolution cutoff at a significantly higher resolution may be justified.

Derivatisation of the crystal with $\mathrm{Sr}^{2+}$ or $\mathrm{Ba}^{2+}$, which are also natural substrates of friulimicin proved unsuccessful, as no crystals were obtained. However, co-crystallisation with iodide yielded crystals comparable in quality to the original ones. It still remains to be established whether these crystals contain any iodide or not.

A higher resolution synchrotron dataset has also been collected at the BL1 beamline at BESSY. Indexing was performed using XDS (Kabsch, 2001), which utilises a similar indexing algorithm implemented in cell_now and therefore had no difficulty in finding the unit cell. XDS was also used for integration and scaling, since crystal mosaicity was relatively high and while processing with other packages was suffering from this property, the integration algorithm in XDS could handle such a case routinely. Data were collected to $1.6 \AA$ resolution, which would allow phase extension and refinement if starting phases could be obtained (Table 5.1).

This crystal thus resisted all attempts to solve the structure so far. However, recent availability of novel data processing programs, a structural model for the related tsushimycin and promising iodide-derivatised crystals may help in overcoming the phase problem and determine the structure. The low resolution of anomalous data is surely a hindrance as a large number of $\mathrm{Ca}^{2+}$-ions should be located in a moderately large unit cell, i.e. the data-toparameter ratio is too low. Search algorithms based on the Patterson-function may perform better in these cases, as direct methods decrease the number of reflections further by selecting the strongest $10 \%$ of the data and may not be optimal for such a problem. 


\begin{tabular}{|c|c|c|c|}
\hline \multirow[b]{2}{*}{ Beamline } & \multicolumn{2}{|c|}{ Friulimicin crystal form I } & \multirow{2}{*}{$\frac{\text { Tsushimycin }}{\text { BL1@BESSY }}$} \\
\hline & In-house & BL1@BESSY & \\
\hline Unit cell parameters & $\begin{array}{c}a=b=73.426 \AA \\
c=105.699 \AA\end{array}$ & $\begin{array}{c}a=b=73.175 \AA \\
c=106.508 \AA\end{array}$ & $\begin{array}{c}a=33.491 \AA, b=36.386 \AA, c=37.518 \AA \\
\alpha=65.64^{\circ}, \beta=68.35^{\circ}, \gamma=69.90^{\circ}\end{array}$ \\
\hline$<\left|E^{2}-1\right|>$ & 1.215 & 0.932 & 0.741 \\
\hline Space group & \multicolumn{2}{|c|}{$\mathrm{P} 3_{1} 21$ or $\mathrm{P} 3_{2} 21$} & $\mathrm{P} 1$ \\
\hline No. of reflections & 449050 & 402457 & 355145 \\
\hline No. of unique reflections & 13029 & 41712 & 75901 \\
\hline Resolution ( $\AA$ ) & $2.40(2.50-2.40)$ & $1.63(1.63-1.75)$ & $1.00(1.10-1.00)$ \\
\hline Completeness & $96.8 \%(80.5 \%)$ & $99.7 \%(99.0 \%)$ & $96.2 \%(92.1 \%)$ \\
\hline Redundancy & $33.37(3.25)$ & $9.62(6.97)$ & $4.50(3.62)$ \\
\hline$<I / \sigma(I)>$ & $21.85(1.74)$ & $9.67(3.05)$ & $9.49(3.35)$ \\
\hline$R_{\text {int }}$ & $0.0813(0.7327)$ & $0.1432(0.6760)$ & $0.1039(0.4990)$ \\
\hline
\end{tabular}

\section{Table 5.1}

Data collection statistics. The significant difference in the $\left\langle\left|E^{2}-1\right|>\right.$ value between the two friulimicin datasets indicates either a deviation from perfect translational symmetry at higher resolution or the failure of profile fitting because of an extensive number of weak reflections in the dataset collected at BESSY. $R_{\mathrm{int}}=\Sigma|I-<I>| / \Sigma I$.

\section{Friulimicin crystal form II}

Not risking possible adverse effects, crystallisation trays with crystals were not taken out from the cold room. A crystal was selected and transferred from the drop into cryoprotectant solution consisting of $0.1 \mathrm{M} \mathrm{NaAc} / \mathrm{HAc} \mathrm{pH}=4.6,14 \%$ isopropanol, $0.14 \mathrm{M} \mathrm{CaCl}_{2}$ and $0.62 \mathrm{M}$ sucrose. After a short soaking time, the crystal was taken out with a loop and dipped into a vessel filled with liquid nitrogen and mounted on a Bruker rotating anode, equipped with Osmic focusing mirrors and Bruker SMART6000 4K CCD diffractometer. The crystal diffracted relatively well, as spots were observed to $1.8 \AA$ resolution. Unit cell determination posed an enormous problem, though. For that purpose, several frames were collected at a comparatively longer distance to promote separation of spots and were thresholded by Proteum. The file containing the spot positions was examined with RLATT and possible unit cell vectors were searched. The spot distribution suggested the presence of a cell vector with $28 \AA$ length, but nothing else could be concluded from the layers. Vector search was then performed with cell_now, using a relatively wide interval (20-220 $\AA$ ) and a fine grid (99999). This resulted in a long list of vectors, which were 
interpreted by hand. As a unit cell vector around $28 \AA$ seemed likely, vectors were searched that made a special angle $\left(90^{\circ}\right.$ or $\left.120^{\circ}\right)$ with this, appeared twice in the list and could be consistently divided into two sets that were related by a single rotation. A vector with a length of $93 \AA$ and another $84 \AA$ long one were found and the initial unit cell thus established were $a=93.3 \AA, b=28.5 \AA, c=84.0 \AA, \alpha=\gamma=90^{\circ}$ and $\beta=123.5^{\circ}$. This cell was then docked by cell_now; three domains were located with increasing figure-of-merit and a sudden drop at the fourth domain. It was therefore concluded that three domains were present. Examination of reciprocal lattice with RLATT revealed not indexed rows of reflections exactly between indexed ones, which hinted that one unit cell vector was twice as long. The final unit cell at that point corresponded therefore to $a=93.1 \AA, b=28.5 \AA, c=168.3 \AA, \alpha=\gamma=90^{\circ}$ and $\beta=123.5^{\circ}$. Integration was performed with all three domains using SAINT, and the resulting data were scaled with TWINABS. Examination with XPREP revealed that reflections corresponding to a C-centred lattice were significantly weaker, and integration was repeated using C-centred lattice setting. A substantial improvement in integration statistics was observed, which confirmed the assumption. Moreover, a C-centred cell with aforementioned parameters could be converted into a $\mathrm{C}$-centred orthorhombic cell with cell parameters $\mathrm{a}=28.5 \AA, b=93.1 \AA$ and $c=140.4 \AA$. This cell was located with cell_now, and the twin law was determined that corresponded to a $180^{\circ}$ rotation about the two diagonals of the $b c$ plane. Unfortunately, this operation does not create a closed group, i.e. rotations about free diagonals of domains would generate an infinite number of cells. In all cases the presence of only three domains could be proved, but it is not known whether only these or other domains are also present that disappear in the noise (Figure 5.5).

This crystal has also been measured at the BL1 beamline at BESSY; diffraction maxima could be observed to atomic resolution. A dataset was collected at a relatively long distance and processing was attempted using XDS. XDS could automatically locate a unit cell corresponding to the primitive setting of the orthorhombic C-cell, and thus confirmed the correctness of the indexing procedure. The dataset has not been processed yet, but the recent availability of programs handling overlapped lattices (Duisenberg et al., 2003) now provides a possibility of solving this crystal structure as well. A dataset extending to atomic resolution could be detwinned accurately enough to provide a starting point for direct methods, which might even solve such a large asymmetric unit considering the high number of $\mathrm{Ca}^{2+}$-ions present in the structure. 


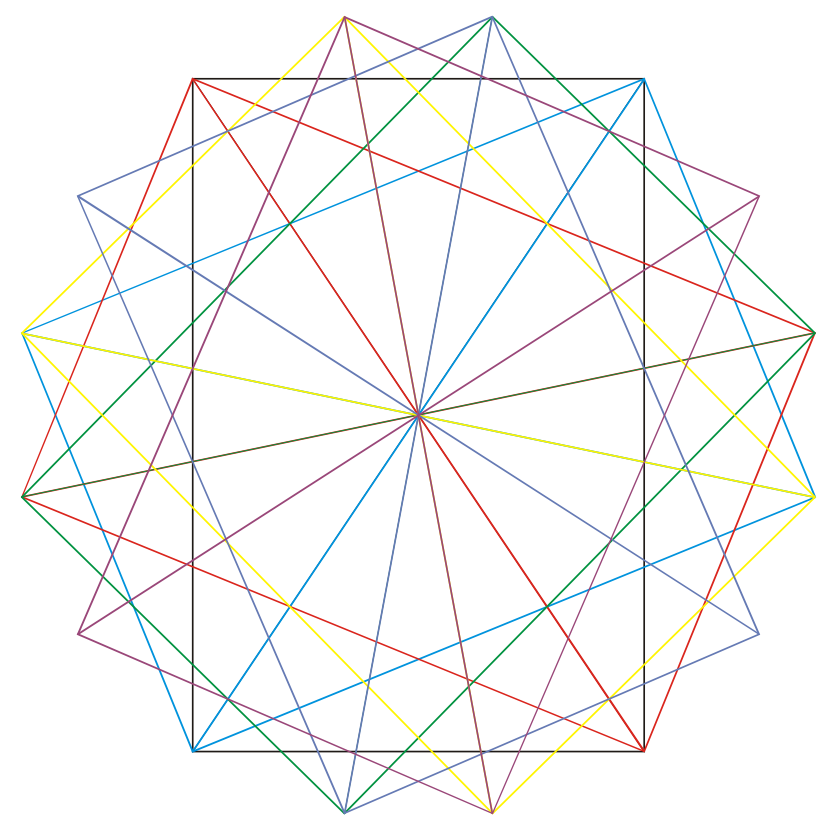

Figure 5.5

Twinning in friulimicin crystal form II with a twofold rotation about the diagonal of the $b c$ plane. Only seven possible domains are shown, but theoretically the operation would generate an infinite series.

\section{Tsushimycin}

Although thin and small, tsushimycin crystals were relatively resistant to mechanical impacts. As crystals grew at room temperature, a suitable one was selected, soaked in cryoprotectant containing $0.1 \mathrm{M} \mathrm{NaAc} / \mathrm{HAc} \mathrm{pH}=4.0,0.05 \mathrm{M} \mathrm{HAc}, 34 \%$ ethanol, $0.6 \mathrm{M}$ 1,6-hexanediol and $0.8 \mathrm{M} \mathrm{CaCl}_{2}$ when necessary, and mounted on our rotating anode. Despite the small crystal size, diffraction was observed to comparatively high resolution, as even at $1.6 \AA$ resolution some spots could be seen. However, the crystal exhibited anisotropic mosaicity, i.e. in certain directions a good-looking diffraction pattern was observed, while in other directions streaks rather than spots (Figure 5.6). Annealing of crystals was tried, but no convincing improvement was observed. Previous experience taught that appropriate integration algorithms could easily handle anisotropic mosaicity, and therefore such a crystal was taken to BESSY and datasets were collected on the BL1 beamline using comparatively thin slicing. The unit cell and space group had already been established in-house of being the lowest possible symmetry, which made a special data collection strategy necessary. It also turned out that the crystal was significantly sensitive to radiation damage and decomposed in the beam after collecting $90^{\circ}$ of rotation range. For these reasons, a new crystal was mounted on a goniometer head equipped with an arc, the beam size was reduced to $0.1 \cdot 0.1 \mathrm{~mm}^{2}$ to 


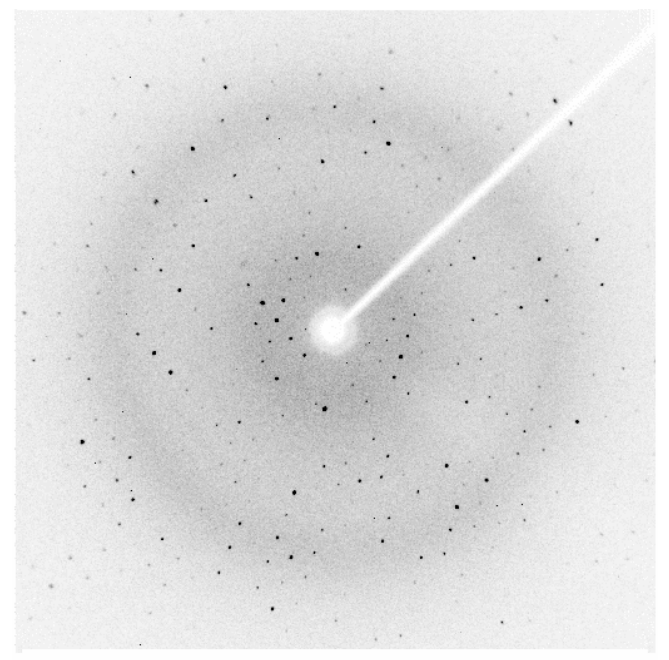

\section{Figure 5.6}

Diffraction frames from tsushimycin showing anisotropic mosaicity. In one orientation highly regular spots are observed, while after $90^{\circ}$ rotation, the spots exhibit an elongated profile. However, modern integration programs can easily handle such property.

spare diffracting volume, and five runs were collected that each covered a rotation range of $90^{\circ}$ and differed in crystal orientation. Between the runs, the crystal was re-centred to irradiate non-damaged parts. In the sixth run, low-resolution reflections were collected with short exposure times to avoid overloads. Processing was performed with XDS, separate runs were scaled together using XSCALE. Statistics generated by XPREP indicated that the data extended to atomic resolution with an $I / \sigma(I)=3.3$ in the highest resolution shell (Table 5.1).

\section{Structure solution}

The cell of tsushimycin is comparatively small, but considering the low symmetry, the size of the asymmetric unit corresponds to that of an average protein crystal. As a comparison, the $\mathrm{P} 1$ form of lysozyme crystallises in a unit cell with $\mathrm{a}=26.65 \AA, b=30.80 \AA, c=33.63 \AA$, $\alpha=88.3^{\circ}, \beta=107.4^{\circ}$ and $\gamma=112.2^{\circ}$, which is about $35 \%$ smaller in volume than the one of tsushimycin $\left(\mathrm{V}=24279 \AA^{3}\right.$ vs. $\left.\mathrm{V}=37708 \AA^{3}\right)$. This crystal form of lysozyme could easily be solved using $a b$ initio direct methods based on data available to $0.95 \AA$; the presence of several sulfur and particularly chlorine atoms together with P1 symmetry support the process. As tsushimycin binds $\mathrm{Ca}^{2+}$-ions and a dataset comparable to that of lysozyme in terms of resolution was available, it was expected that structure solution would work as plainly as for lysozyme. 
Previous experience suggested that $a b$ initio direct method solution works best if a small fragment (preferably the heavy atoms) is located first with dual space recycling and then the structure is expanded using peaklist optimisation. Thus, a SHELXD (Sheldrick et al., 2001) job corresponding to this strategy was set up that instructed the program to find 30 atoms in the dual-space recycling (FIND 30), reject tries if the correlation coefficient is below a certain threshold (TEST 15 5) and then expand the structure with peaklist optimisation from 30 to 1300 atoms in ten steps. An immediate solution has been achieved: all tries out of the performed nine led to the right solution (and therefore no bimodal distribution has been obtained). As one try required about 2 minutes of CPU times on a $2.8 \mathrm{GHz}$ Intel Xeon, this corresponds to a solution rate of about 30 solutions/hour, which is much higher than those observed for cephaibols (about 0.5 solution/hour) not to mention that for feglymycin ( 0.016 solution/hour) although tsushimycin is two- to tenfold larger. The presence of heavier atoms therefore assists substantially the structure solution and hints that under certain circumstances structures containing a few heavier elements besides the protein could be solved even if data do not extend to atomic resolution.

\section{Model building and refinement}

The structure provided by SHELXD was incomplete and about $20 \%$ of atoms were missing. First, peaks were assigned corresponding to peptide atoms according to the sequence reported by Vértesy et al. (2000) using the program XP. To build up the missing part of the structure, a map was created using SHELXE (Sheldrick, 2002) starting from the direct method solution and specifying a relatively low solvent content (25\%). A very clear map was obtained and missing atoms were added manually using the program XtalView (McRee, 1999). The structure was refined against all $F_{o}^{2}$ with no intensity cutoff using SHELXL (Sheldrick \& Schneider, 1997). Throughout the refinement, bond length, angle distance, chiral volume and planarity restraints were imposed on appropriate atoms. As most of the residues are not commonly found in proteins, bond length and bond angle distances were generated using small molecules from the CSD (Allen, 2002). When no suitable fragment was found (e.g. for isotetradecenoic acid), restraints were generated using a table compiled by Allen et al. (1992) listing common bond lengths found in organic compounds. Solvent atoms were added using SHELXWAT and manually. All non-hydrogen atoms were refined anisotropically with suitable rigid bond and similarity restrains. For solvent waters, approximately isotropic restraints were also employed. Hydrogen atoms were included in the later stages of 


\begin{tabular}{ll}
\hline Resolution range $(\AA)$ & $26.02-1.00$ \\
$R_{1}[\mathrm{~F}>4 \sigma(\mathrm{F}) /$ all data $]$ & $0.1318 / 0.1464$ \\
$R_{\text {free }}[\mathrm{F}>4 \sigma(\mathrm{F}) /$ all data $]$ & $0.1640 / 0.1791$ \\
No. of parameters & 11407 \\
No. of restraints & 12261 \\
No. of peptide atoms & 1069 \\
No. of non-H atoms & 1283 \\
Solvent content & $40.0 \%$ \\
R.m.s.d. from ideal geometry & \\
\multicolumn{1}{c}{ Bond lengths $(\AA)$} & 0.014 \\
$\quad$ Angle distances $(\AA)$ & 0.033 \\
$\quad$ Common planes $(\AA)$ & 0.430 \\
\hline
\end{tabular}

Table 5.2

Summary of final refinement for tsushimycin.

refinement. About 5\% of the reflections (selected in thin shells) were omitted from the refinement and reserved for the calculation of $R_{\text {free. }}$ Refinement statistics are shown in Table 5.2.

\section{STRUCTURE DESCRIPTION}

\section{Amino acid residues and sequence}

The absolute configuration of tsushimycin was assigned based on the configuration of chiral centres established by Bodanszky et al. (1969). Accordingly, aspartate, $\beta$-methylaspartate, proline and valine residues are present as L-enantiomers, the pipecolinic acid as D-enantiomer, while from 2,3-diaminobutyric acid two diastereomers are present: Dab2 is the L-threo-form, while Dab10 is D-erythro. This was in good agreement with the X-ray structure, although the configuration of $\mathrm{C}^{\beta}(\mathrm{Dab} 10)$ could not be determined unambiguously, as the scattering properties of carbon and nitrogen are very similar, and it proves to be problematic to distinguish these elements even at such high resolution. However, characteristics in the chemical environment allowed identification of $\mathrm{N}^{\gamma}(\mathrm{Dab} 10)$, since it was found to be closely situated to two charged aspartate sidechains (Figures 5.12 and 5.13). 
On the other hand, the high resolution allowed unambiguous determination of atom connectivity. The measurement confirms the correctness of one of the two structures proposed originally by Fujino (1965), which has recently been supported by spectroscopic evidence (Vértesy et al., 2000; Kong \& Carter, 2003). The sequence of tsushimycin can therefore be written as:

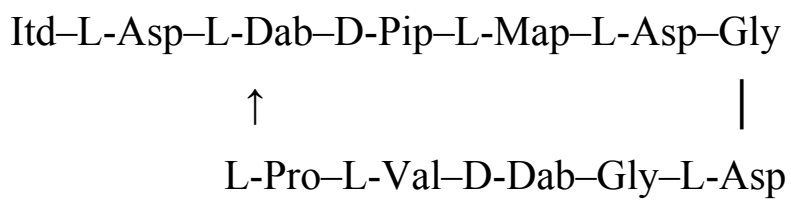

The high number of aspartates common in $\mathrm{Ca}^{2+}$-binding proteins is noteworthy, together with the Asp-Gly pattern that occurs twice in the peptide ring. This leads to decreased stability under physiological conditions with half-lifes in the order of one week. The structure of the fatty acid sidechain could also be confirmed together with the geometry of the double bonds, which is present as the $c i s$-form.

\section{Conformation}

In the asymmetric unit, twelve independent molecules are present. Each molecule binds a $\mathrm{Ca}^{2+}$-ion and association of the molecules is mediated by additional $\mathrm{Ca}^{2+}$-ions. Peptide atoms are well defined in the electron density and only a handful number of discretely disordered sidechains were found. On the other hand, most of the fatty acid residues are continuously disordered, although there are molecules whose long alkyl chains are clearly visible. Crystal packing is tight, but there are large cavities among the molecules, filled with water or with disordered alkyl chains.

\section{Overall structure}

The molecules were found to form a globule with the $\mathrm{Ca}^{2+}$-ion in the middle and the peptide chain covering it. The backbone conformation resembles that of $\mathrm{K}^{+}$-bound valinomycin, i.e. a low-order Lissajoux-curve, taking into account that the cyclopeptide core in valinomycin consists of 12 residues and therefore it comprises three "waves" (Duax et al., 1996), while tsushimycin contains only two (Figure 5.7). Valinomycin exhibits a regular alteration of amino acid chirality according to the pattern -(D-L-L-D)-, but the presence of such a pattern cannot be demonstrated in tsushimycin, although the Gly residues would allow some degree of freedom in assigning chiralities in order to obey this regular alternation.

Conformations of independent molecules were compared by identifying rigid atoms with ESCET (Schneider, 2002), fitting these rigid atoms on each other, transforming the 


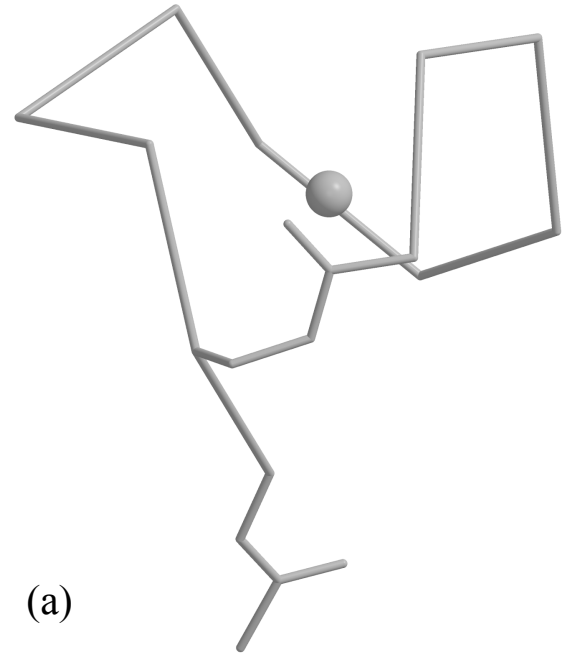

(b)

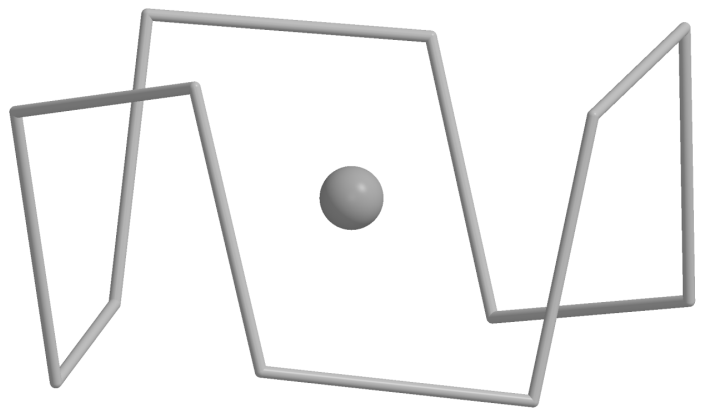

\section{Figure 5.7}

Backbone traces of (a) tsushimycin and (b) valinomycin showing homologous backbone conformations. The long fatty acid sidechain of tsushimycin has been truncated for clarity.

whole molecule with the determined operation and evaluating the results graphically. Although a high degree of conformational diversity was expected, surprisingly good agreement was found among independent molecules: the cyclopeptide ring adopts a nearly identical conformation in all 12 cases, and even the floppy sidechains are in a highly homologous arrangement (Figure 5.8). This finding suggests that the structure of the antibiotic with a bound $\mathrm{Ca}^{2+}$ is fairly well defined. Closer examination allowed classification of molecules into two classes, based on the sidechain conformation of Asp1.
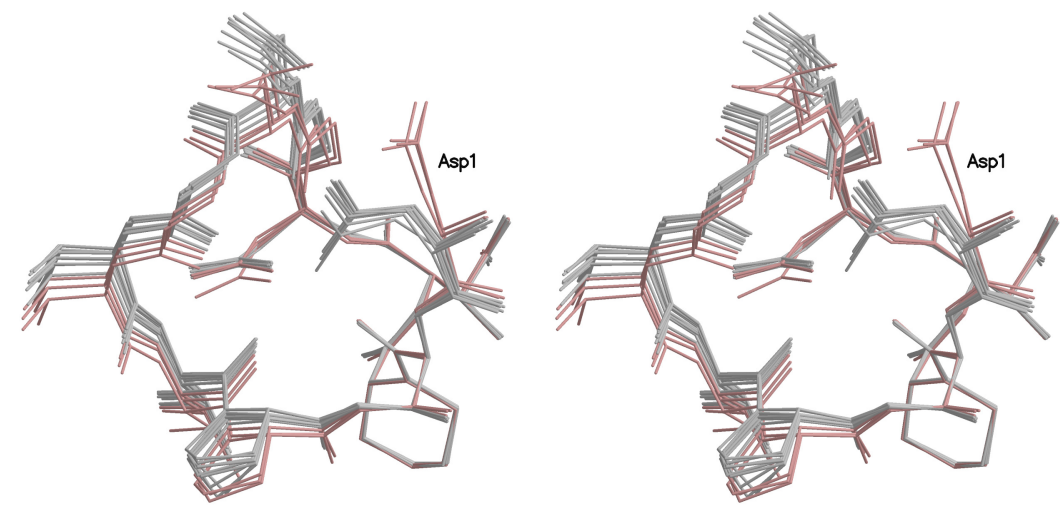

\section{Figure 5.8}

Stereo view of the superposition of all 12 independent tsushimycin molecules. Molecules belonging to different conformation classes are distinguished by their colour (class I molecules are grey, class II molecules are redbrown). The aspartate sidechain (Asp1) decisive in the assignment of conformation class is marked with a label. 


\section{Calcium binding}

The $\mathrm{Ca}^{2+}$-ion present in the central cavity of the molecules is coordinated by several oxygen atoms. Four of these are backbone carbonyl oxygens, one of them is from a water molecule, and one or two carboxyl oxygens from aspartate sidechains complete the coordination shell. The aforementioned two conformation classes also differ in the number of oxygen atoms binding to the $\mathrm{Ca}^{2+}$-ion, since the sidechain of Asp1 also participates in $\mathrm{Ca}^{2+}$ coordination in one class (class I), while it is not sufficiently near in the remaining one (class II). It is surprising that in spite of the presence of several aspartate sidechains, mainchain carbonyl atoms play a decisive role in $\mathrm{Ca}^{2+}$-binding. The arrangement of these corresponds to that in valinomycin, although in valinomycin there are six such oxygen atoms, while in tsushimycin there are only four, corresponding to two coordinating mainchain carbonyl oxygens in each "wave" of the Lissajoux-curve.

In molecules belonging to class $\mathrm{I}$, seven oxygen atoms coordinate the $\mathrm{Ca}^{2+}$-ion with pentagonal bipyramidical geometry (Figure 5.9). Calcium-oxygen distances vary in the range 2.28-2.48 $\AA$, with the water molecule being the most distant, while oxygen atoms from the antibiotic being nearer to the $\mathrm{Ca}^{2+}$-ion. Equatorial positions are occupied by backbone carbonyl oxygens $\mathrm{O}(\mathrm{Dab} 2), \mathrm{O}(\mathrm{Gly} 6), \mathrm{O}(\mathrm{Gly} 8), \mathrm{O}(\mathrm{Val10})$ and the water molecule; the aspartate carboxyl oxygens $\mathrm{O}^{\delta}(\mathrm{Asp} 1)$ and $\mathrm{O}^{\delta}(\mathrm{Asp} 5)$ are in axial positions, probably because of the larger sterical demand of the carboxyl group. Axial oxygens are found to be nearer to
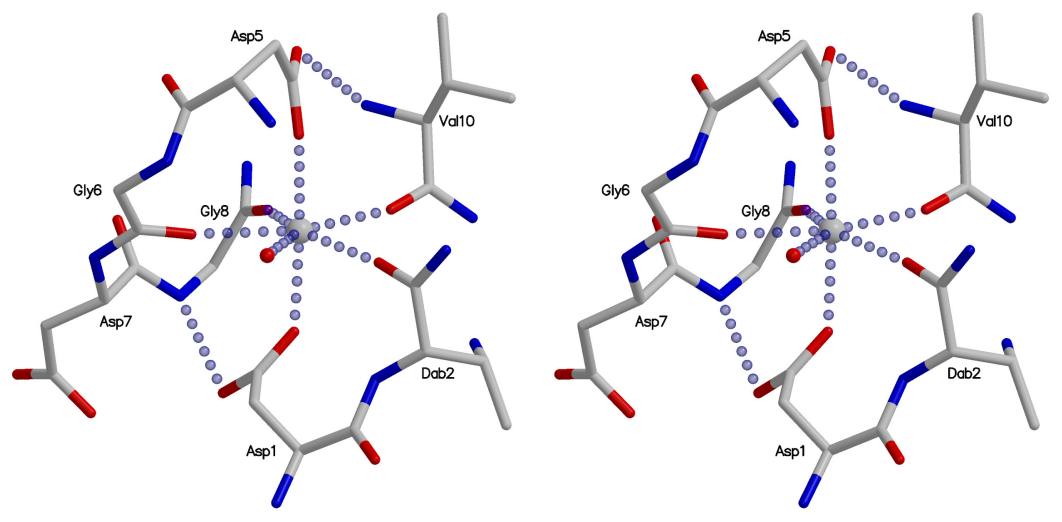

Figure 5.9

Stereo view of calcium binding in class I molecules. The $\mathrm{Ca}^{2+}$-ion is coordinated by seven oxygen atoms according to pentagonal bipyramidal geometry. Sidechains of Asp1 and Asp5 are in axial positions, while the water molecule and mainchain carbonyl oxygens are equatorial. Residues not participating in the interaction are not shown. 

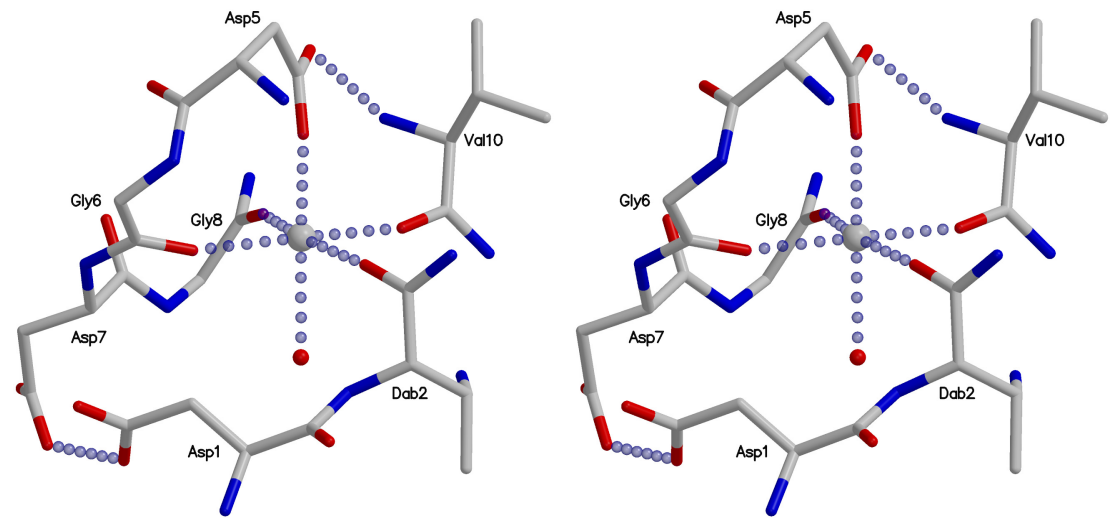

\section{Figure 5.10}

Stereo view of calcium binding found in class II molecules. The $\mathrm{Ca}^{2+}$-ion is now surrounded by six oxygen atoms according to octahedral geometry. The sidechain of Asp1 is displaced and cannot participate in the binding, but is substituted by a water molecule. Residues not taking part in the interaction have been omitted for clarity.

the $\mathrm{Ca}^{2+}$-ion (2.36 and $2.28 \AA$ for Asp1 and Asp5, respectively) than equatorial ones, which suggests that these groups are deprotonated and negatively charged at the $\mathrm{pH}$ of the crystallisation. Bond angles between axial and equatorial oxygens are in the range $83-98^{\circ}$, while those between equatorial groups vary between $65-79^{\circ}$, with that between the water molecule and neighbouring carbonyl oxygens being the smallest, in accordance with the water oxygen being farther away.

On the other hand, class II molecules bind the $\mathrm{Ca}^{2+}$ according to a regular octahedral geometry (Figure 5.10). The carboxyl oxygen of Asp1 is displaced and does not participate in $\mathrm{Ca}^{2+}$-binding. However, it is substituted by a water molecule, while the equatorial water molecule present in class $\mathrm{I} \mathrm{Ca}^{2+}$-coordination is missing. Remaining coordinating atoms keep their positions, although a slight closure of the peptide chain can be observed, as distances from the $\mathrm{Ca}^{2+}$-ion are now $2.26 \AA$ (for the carboxyl oxygen from Asp5) or between 2.30$2.36 \AA$ (carbonyl oxygens). The water molecule with a binding distance of $2.53 \AA$ is significantly more distant than any of the other coordinating atoms. There are slight changes in the bond angles: the one between "equatorial" substituents and "axial" ones is between $86-97^{\circ}$, while angles between "equatorial" atoms vary in the range $80-106^{\circ}$, the largest being between $\mathrm{O}(\mathrm{Dab} 2)$ and $\mathrm{O}(\mathrm{Gly} 6)$, where the equatorial water in class I molecules binds. It may also be speculated that the water molecule in this case is in fact a chloride to balance the charges, but this is not likely, since refined as water, temperature factors of the atom in question lies in the range observed for neighbouring water molecules. 
(a)

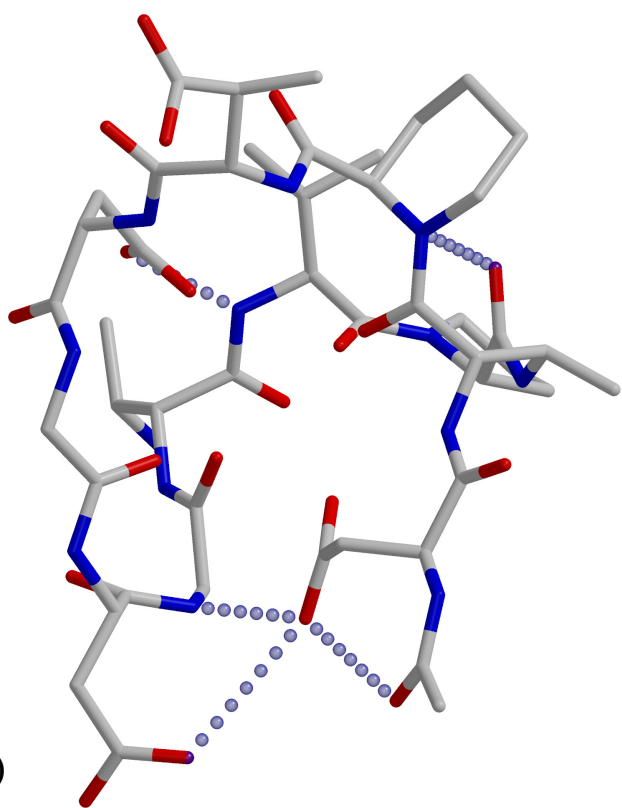

(b)

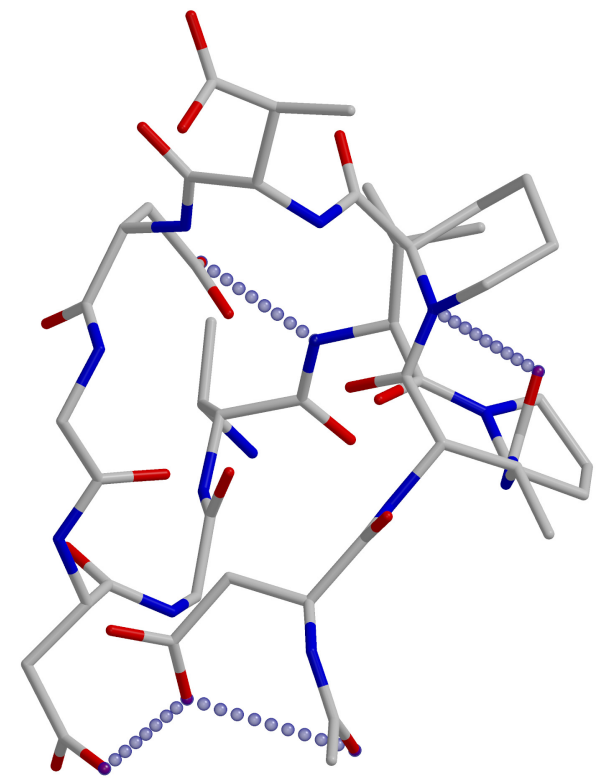

\section{Figure 5.11}

Secondary interactions that do not involve the central $\mathrm{Ca}^{2+}$-ion in class I (a) and class II (b) molecules. The sidechain of Asp1 (bottom of the picture) can be stabilised in two distinct conformations. The $\mathrm{Ca}^{2+}$-ion has been omitted to achieve a clearer view.

The two binding modes may also be informative about the process of entry or exit of the $\mathrm{Ca}^{2+}$-ion, which may happen by three mechanisms. The displacement of the sidechain of Asp1 opens up the binding pocket, so $\mathrm{Ca}^{2+}$ can enter or leave, and a similar process involving the sidechain of Asp5 is also possible. An entry path between $\mathrm{O}(\mathrm{Dab} 2)$ and $\mathrm{O}(\mathrm{Gly} 6)$ can also be considered, as in this case a pre-formed octahedral binding formation would attract the ion and the gap between the oxygen atoms is large enough to accommodate a water molecule; an exit via this mechanism is unlikely, though.

\section{Secondary interactions}

Apart from interactions involved in coordination of $\mathrm{Ca}^{2+}$, only a few stabilising hydrogen bonds can be seen. In class I molecules, strong hydrogen bonds fix carboxyl groups of Asp1 and Asp5 to amide nitrogens of Gly8 and Val10, respectively, via carboxyl oxygens not participating in $\mathrm{Ca}^{2+}$-coordination. Sub-van der Waals distances likely to correspond to weak hydrogen bonds can be found between $\mathrm{O}^{\delta}(\mathrm{Asp} 1)-\mathrm{O}^{\delta}(\mathrm{Asp} 7)$ and $\mathrm{O}^{\delta}(\mathrm{Asp} 1)-\mathrm{O}(\mathrm{Itd} 0)$ with about $3.3 \AA$ distance between non-hydrogen atoms, while the one between N(Pip3)-O(Pro11) is more likely to be a dipole-dipole interaction, although the binding distance is similar (Figure 5.11). In class II molecules, $\mathrm{O}^{\delta}(\mathrm{Asp} 5)$ is $2.9 \AA$ away from $\mathrm{N}(\mathrm{Val10})$, but no interaction between $\mathrm{O}^{\delta}(\mathrm{Asp} 1)$ and $\mathrm{N}(\mathrm{Gly} 8)$ can be observed. However, $\mathrm{O}^{\delta}(\mathrm{Asp} 1)$ is now 
connected to $\mathrm{O}^{\delta}(\mathrm{Asp} 7)$ via a stronger interaction than in class I molecules, as the binding distance is reduced to $2.8 \AA$ from the previous $3.3 \AA$. Interactions between $\mathrm{O}^{\delta}(\mathrm{Asp} 1)-\mathrm{O}(\mathrm{Itd} 0)$ and N(Pip3)-O(Pro11) are also present in class II molecules with bond lengths similar to class I molecules. The fact that the sidechain of Asp1 can be fixed in two conformations supports the entry and exit-mechanisms in that this moiety acts as the gate. The long alkyl chain of Itd0 is pointing away and does not interact with hydrophobic residues on the peptide core.

\section{Crystal packing}

The packing of tsushimycin molecules in the unit cell is not homogeneous. There are large regions that seem to be empty, while in other parts molecules concentrate. It is interesting to note that there seems to be polar and apolar cavities in the crystal, and the molecules act as separators between them. Considering also the shape of the apolar cavity, which resembles a sphere, one might describe the unit cell as a micelle, with hydrophobic sidechains pointing inwards and polar peptide heads being on the surface. This micelle is slightly distorted, as in one direction there are channels between neighbouring hollows and therefore it is better described as a string of pearls. As the unit cell has similar cell dimensions in all three directions, the micelle is nearly isometrical.

\section{Interaction surfaces}

Although the packing is tight, only a limited number of specific interactions between the molecules can be seen. There seem to be three well-defined regions in the sequence, with which these molecules interact, although there are considerable differences in their extent. The first and largest region involves three residues and seems to be decisive in determining whether a molecule belongs to conformation class I or class II, while the remaining two surfaces consist of one residue. As there are 12 independent molecules in the unit cell, but intermolecular interactions seem to be highly homologous, a relative notation will be used in the following to distinguish residues, i.e. molecules will be numbered chronologically as they appear in the text.

For class I molecules, $\mathrm{O}^{\delta}(\mathrm{Asp} 1 / 1), \mathrm{O}(\mathrm{Itd} 0 / 1)$ and $\mathrm{O}^{\delta}(\mathrm{Asp} 7 / 1)$ coordinate a $\mathrm{Ca}^{2+}$-ion according to octahedral geometry (Figure 5.12). The octahedron is completed by $\mathrm{O}^{\delta}(\mathrm{Map} 4 / 2)$ from a second molecule and two additional water molecules. Oxygen atoms from antibiotic molecules are in "equatorial" positions, while the waters are "axial". Bond lengths vary between 2.28-2.39 $\AA$; the peptide atoms were found to occupy a closer position, while the waters are further away. The variation in bond angles is not as extensive as the one found for 


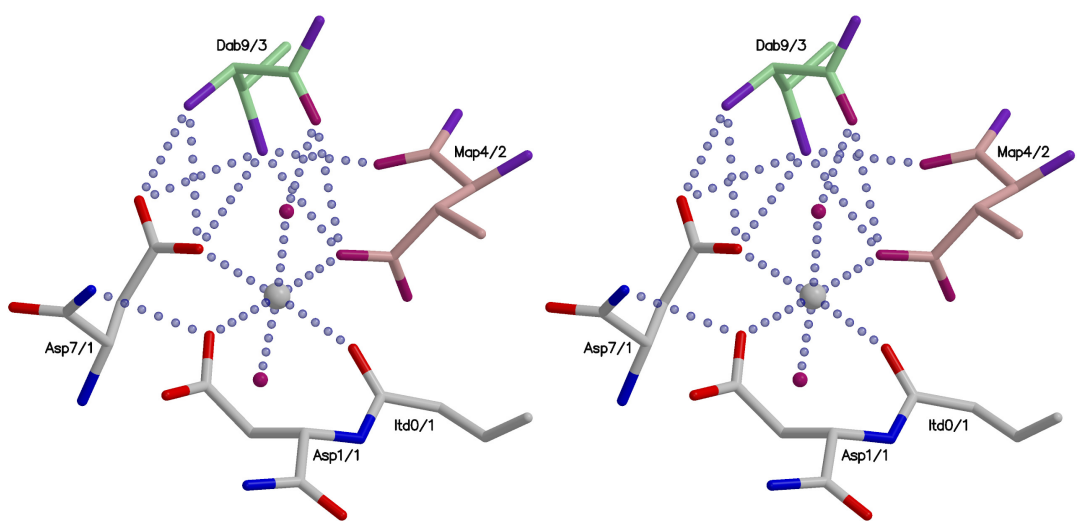

\section{Figure 5.12}

Stereo view of the $\mathrm{Ca}^{2+}$-mediated intermolecular association surface in conformation class I. Although the secondary interactions are not too strong, their number may compensate and provide relatively high stability to the interface.

the central $\mathrm{Ca}^{2+}$, and therefore the octahedron is nearly regular. These interactions are supplemented by hydrogen bonds and salt bridges between the $\mathrm{O}^{\delta}$ atoms of Asp $7 / 1$ and either $\mathrm{N}^{\gamma}\left(\right.$ Dab9/3) or $\mathrm{N}(\mathrm{Dab} 9 / 3)$ from a third molecule, the $\mathrm{O}^{\delta}(\mathrm{Map} 4 / 2)-\mathrm{N}^{\gamma}(\mathrm{Dab} 9 / 3)$, the $\mathrm{O}(\mathrm{Map} 4 / 2)-\mathrm{N}^{\gamma}(\mathrm{Dab} 9 / 3)$ and the $\mathrm{O}^{\delta}(\mathrm{Map} 4 / 2)-\mathrm{O}(\mathrm{Dab} 9 / 3)$ hydrogen bonds. One of the axial water molecules is kept in position by a hydrogen bond to $\mathrm{O}(\mathrm{Dab} / 3)$. Interactions involving $\mathrm{N}^{\gamma}$ (Dab9) proved helpful in assigning the chemical element and thereby the chirality of $C^{\beta}$ (Dab9), as $C^{\gamma}$ (Dab9) is not likely to be involved in such interactions. The exact strength of these interactions vary slightly among independent molecules, but it is consistent that $\mathrm{O}(\mathrm{Map} 4 / 2)-\mathrm{N}^{\gamma}(\mathrm{Dab} 9 / 3)$ and one of the two $\mathrm{O}^{\delta}(\mathrm{Asp} 7 / 1)-\mathrm{N}(\mathrm{Dab} 9 / 3)$ are the stronger ones with distances around $2.9 \AA$. The remaining interactions are between 3.2-3.3 $\AA$ in bond length, but as they have a salt bridge character, where the distance-dependence does not show such a sharp cutoff, there may still be relatively strong connections between the molecules.

The interface in class II molecules looks similar, apart from the fact that the sidechain of Asp1 now occupies an "axial" position in the octahedron, while one of the waters is displaced to become "equatorial" (Figure 5.13). However, there seems to be no bound $\mathrm{Ca}^{2+}$ in the centre of the octahedron, only a water molecule. This can be concluded from the bond distances, which are in the order of 2.5-2.8 $\AA$ in this case. Moreover, assigning the chemical element as $\mathrm{Ca}^{2+}$, the occupancy refines to $40 \%$, which nearly exactly corresponds to a fully occupied water molecule. Remaining connections involved in the interface are analogous to those in class I molecules. The "equatorial" water molecule is also hydrogen-bonded to N(Gly8/1), 

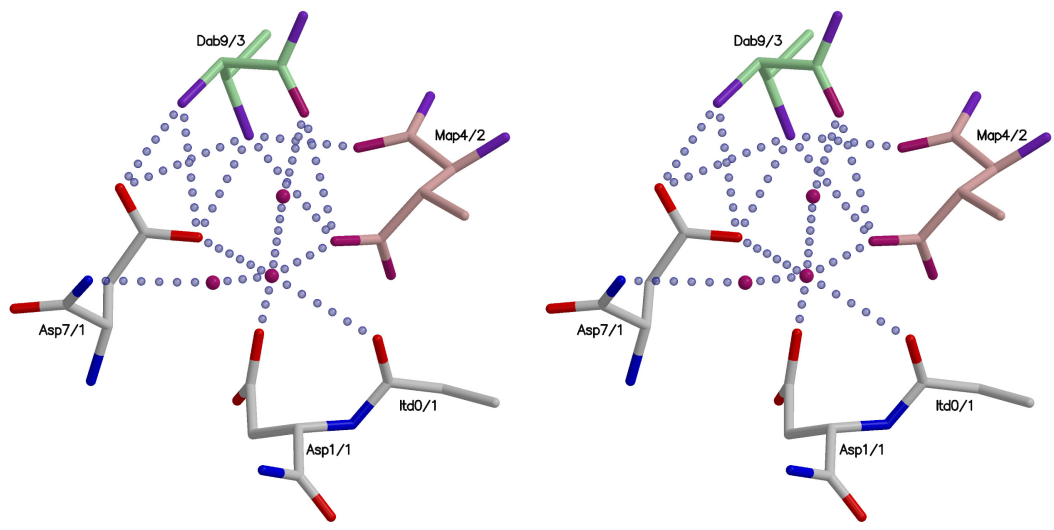

Figure 5.13

Intermolecular connection in molecules from conformation class II. The octahedrally coordinated atom is likely to be a water molecule based on bond distances and temperature factors. This interface is therefore less stable than the one found in class I molecules, but may still be strong enough to keep the molecules in a dimer.

which binds $\mathrm{O}^{\delta}(\mathrm{Asp} 1)$ in class I molecules. Since distances from the central water molecule are longer, the octahedron is larger and antibiotic molecules are marginally further apart.

As molecules associate in a highly homologous way, Map4 and Dab9 from molecule 1 are also involved in intermolecular interactions. These amino acids take part in the same type of interface, and play analogous roles to what has been described above for Map4/2 and Dab9/3. Therefore, each interface connects three molecules, and each molecule participates in three such interfaces.

\section{Association}

Careful examination of molecular packing reveals a higher degree of order among the molecules. Although each molecule participates in three intefaces, molecule 1 and molecule 3 are connected twice by acting symmetrically in two intefaces, i.e. in one interface, molecule 1 binds the $\mathrm{Ca}^{2+}$ and molecule 3 participates via $\mathrm{N}^{\gamma}$ (Dab9), while in the other one molecule 3 coordinates the $\mathrm{Ca}^{2+}$ and molecule 1 takes part with its $\mathrm{N}^{\gamma}(\mathrm{Dab} 9)$. This results in a dimer in which the monomers are related by a non-crystallographic twofold axis (Figure 5.14). These dimers are then connected to other dimers via sidechains of Map4 residues present in both chains. A dimer therefore is connected to four other dimers, as it donates two and accepts two Map4 carboxyl groups, leading to heavily interconnected crystal packing. The noncrystallographic twofold axis is oriented in a way that both Itd0 residues point to the same direction, therefore separates the polar and the apolar surface of the dimer. The long alkyl 


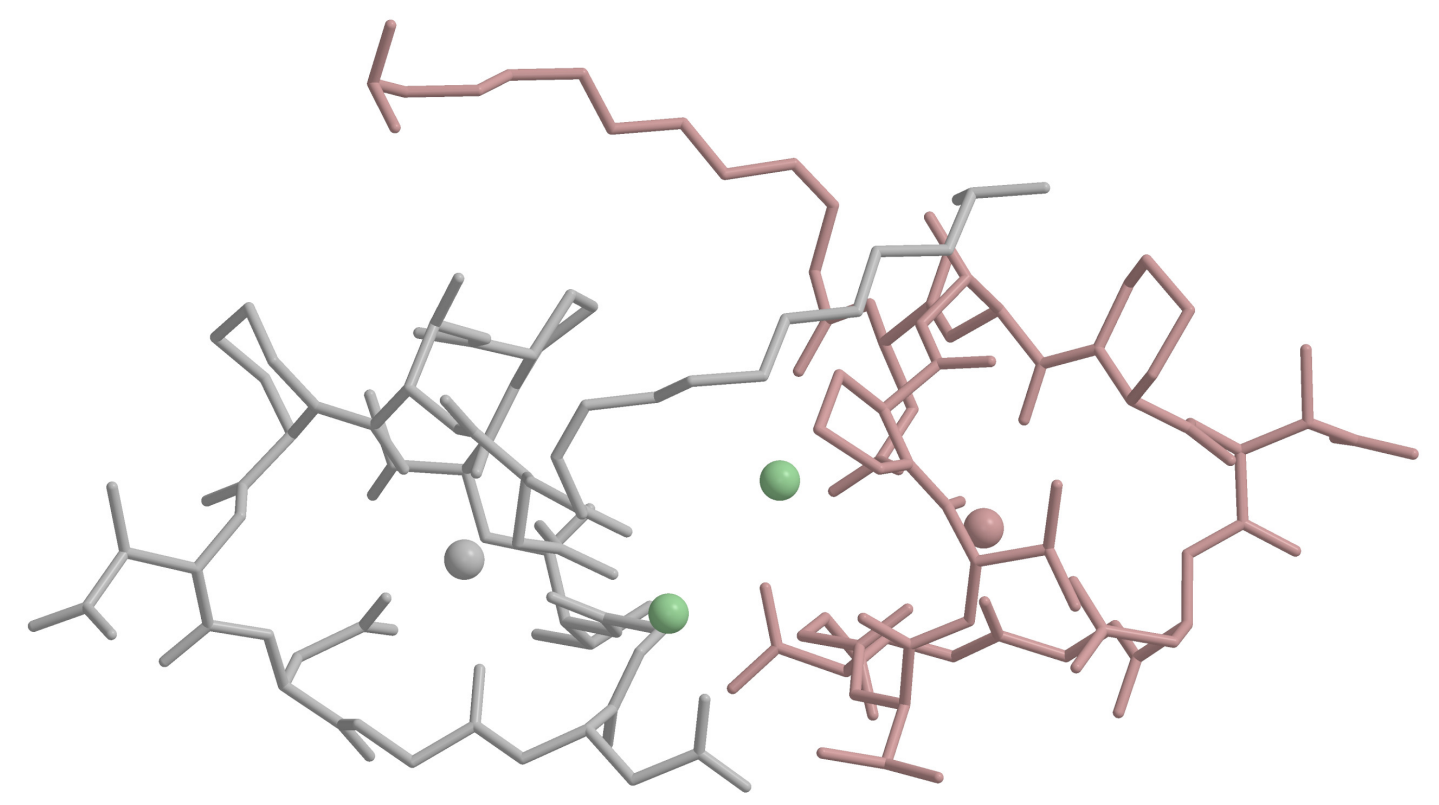

\section{Figure 5.14}

Side view of a tsushimycin dimer. It is noteworthy that all aspartate sidechains point to the bottom and constitute a hydrophilic surface there, while the opposite side of the molecule is predominantly hydrophobic. The green $\mathrm{Ca}^{2+}$-ions are involved in dimer interaction and occupy a slightly off-centre position. The opening of the intermolecular tunnel is therefore wide enough to allow the binding of a long polymer chain. The residues on the two side extremes of the dimer are the $\beta$-methyl-aspartates (Map4) that also take part in the intermoleculer interface and link dimers like a joint.

chains can then interact with each other or with hydrophobic surfaces on the other molecule thereby stabilising the structure.

Although missing the $\mathrm{Ca}^{2+}$-ion in their interface, class II molecules can also participate in dimer formation, since mixed class I-class II dimers were found to have a structure nearly identical to pure class I-class I dimers. No class II-class II dimers were found, though. This may arise from the reduced strength of the class II interface or may simply be accounted for by statistics as there are nine class I and three class II molecules in the cell and the probability of pairing two class II molecules with each other is low.

\section{Binding clefts}

Dimerisation results in the formation of a tunnel between monomers. The two sides of the tunnel is composed of hydrophobic surfaces of the two molecules, the bottom is made up of the binding interfaces, while the top can be opened or closed by the long alkyl sidechains (Figure 5.15). The tunnel is about $9 \AA$ long and 5-8 $\AA$ wide, non-polar in the middle and 


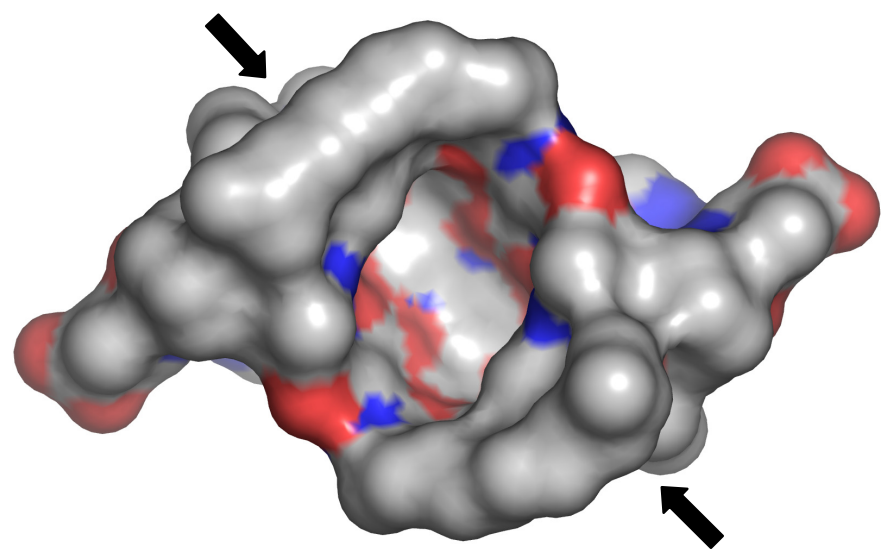

\section{Figure 5.15}

Top view of a tsushimycin dimer showing the intermolecular tunnel and an opening at its centre, which would provide space for sidechains connected to the lipoteichoic acid polymer bound in the binding cleft. The two sides of the tunnel are marked with arrows.

charged at the two ends because of incorporated $\mathrm{Ca}^{2+}$-ions. The formation of the cleft requires therefore the presence of $\mathrm{Ca}^{2+}$-ions, and can be anchored to the cell membrane with the long alkyl sidechains.

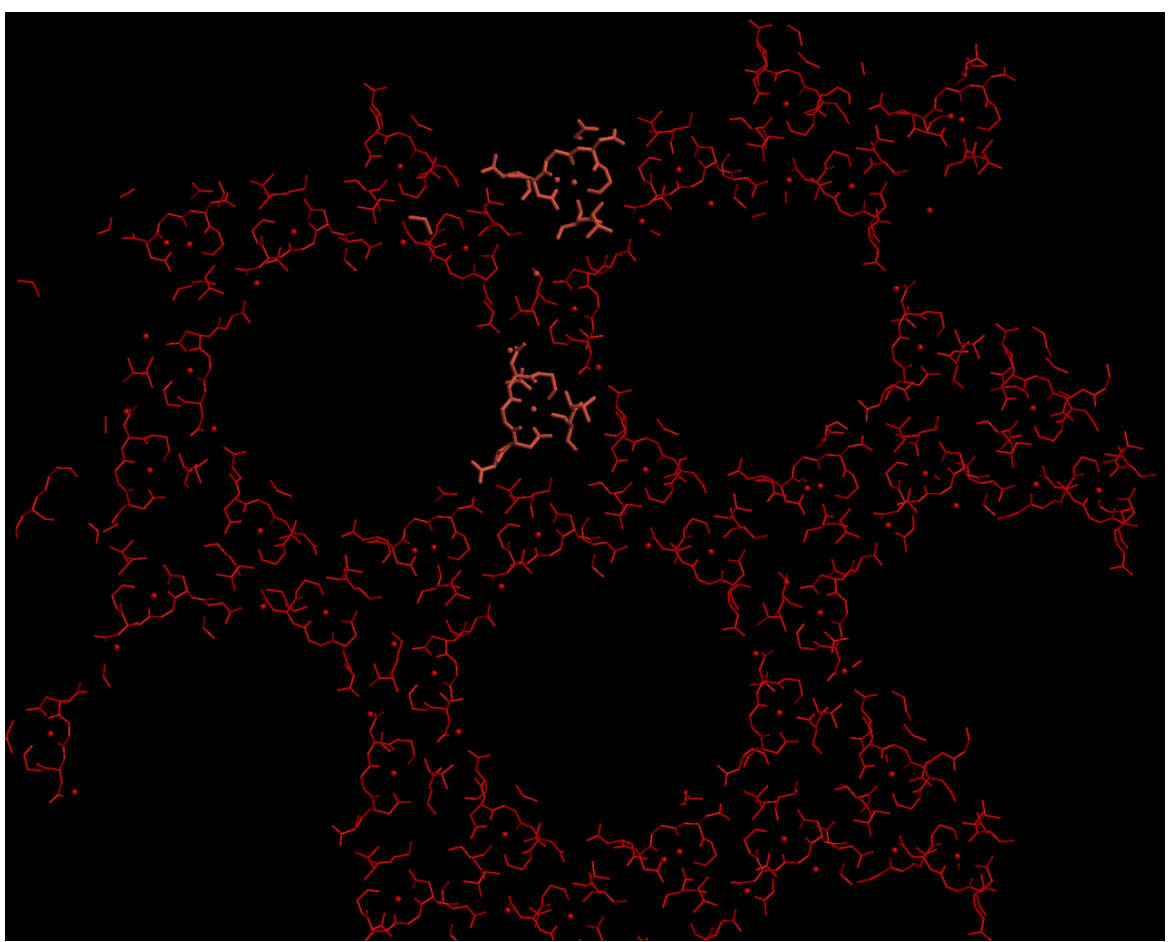

Figure 5.16

Packing in tsushimycin crystals. The molecules constitute spheres, which are hydrophobic inside and hydrophilic outside and may be regarded as small micelles. 


\section{Micelles}

The dimers in the unit cell were found to be oriented in a way that their long fatty acid sidechains point into a large cavity. Apart from visible parts of these alkyl chains, no electron density can be found in this hollow, which therefore can be regarded as empty. Charged groups of molecules are all pointing outside and constitute a polar surface. This arrangement resembles the structure of a putative micelle, which is elongated in one dimension because of slightly different unit cell dimensions (Figure 5.16). Each unit cell consists of a micelle, which is connected to other ones through structural waters that are well defined in the density; there seems to be about three layers of waters between neighbouring conglomerates, but a single $\mathrm{Ca}^{2+}$-ion was also identified as a link between two adjoining micelle surfaces.

\section{BIOLOGICAL IMPLICATIONS}

Lipopeptide antibiotics constitute a highly promising group of drugs against resistant bacteria, but little is known about their exact biological action and even the chemical structure has not been unambiguously established. High resolution X-ray crystal structure determination of the prominent member tsushimycin confirmed the chemical structure proposed by Vértesy et al. (2000), and may also give hints in clarifying the mechanism of antimicrobial potency.

\section{$\mathrm{Ca}^{2+}$-ions and biological action}

\section{Structure stabilisation}

The conformational uniformity of the antibiotic is strongly dependent on the presence of $\mathrm{Ca}^{2+}$-ions. It was found that apart from those involving the central $\mathrm{Ca}^{2+}$-ion, there are very few secondary interactions that stabilise the conformation observed in the crystal structure. The central $\mathrm{Ca}^{2+}$-ion plays therefore a decisive role in determining the conformation of the molecule. Addition of $\mathrm{CaCl}_{2}$ to the antibiotic induces a significant decrease in solubility of the antibiotic; since most of the carboxyl groups remain on the antibiotic surface even in $\mathrm{Ca}^{2+}$-bound form, the reduction in solubility may be a consequence of a remarkable drop in conformational diversity. It is also noteworthy that in spite of the presence of several aspartate residues, which are conserved even in distant analogues, the $\mathrm{Ca}^{2+}$ is coordinated primarily by mainchain carbonyl oxygens, although two aspartate residues also take part in the binding. 
It is therefore plausible to assume that the antibiotic has no well defined conformation in the absence of $\mathrm{Ca}^{2+}$, and consequently cannot exert its inhibitory effect. Upon addition of $\mathrm{Ca}^{2+}$ the active structure is stabilised by supplementary interactions and the conformational diversity exhibited without $\mathrm{Ca}^{2+}$ is reduced and becomes remarkably uniform.

\section{Dimerisation and toxicity}

The dimers found in the crystal structure are also strongly dependent on the presence of $\mathrm{Ca}^{2+}$-ions, as each intermolecular interface involves the presence of such an ion. Dimerisation also occurs if one of the $\mathrm{Ca}^{2+}$-ions is missing, as demonstrated by the existence of mixed class I-class II dimers, which miss one $\mathrm{Ca}^{2+}$-ion; however, it is not clear, whether class IIclass II dimers not involving $\mathrm{Ca}^{2+}$ ions in dimer interactions can also exist.

It is instructive to compare tsushimycin with friulimicin, which differ only in one position in the sequence: instead of an aspartate, friulimicin possesses asparagine as the first residue (Figure 5.1). This small difference results in a significant decrease in toxicity without affecting antibacterial potency. This aspartate residue coordinates two $\mathrm{Ca}^{2+}$-ions in tsushimycin class I molecules; however, in friulimicin it can coordinate only one, as one of its oxygen atoms is replaced by nitrogen. It may be inferred that the binding of $\mathrm{Ca}^{2+}$ in the connecting interface is therefore partially responsible for toxicity. However, as there is no structure available for friulimicin, it cannot be decided whether dimerisation of antibiotic molecules is also affected by the replacement of Asp1 with Asn1.

\section{Physiological role of association}

\section{Dimerisation}

As all the molecules present in the crystal structure of tsushimycin were found to compose dimers, it can be assumed that this unit may be relevant to biological action. The dimers are best described as amphipatic molecules, since one of their sides is predominantly hydrophilic because of the presence of charged carboxyl groups, while the opposite side exposes mostly hydrophobic surfaces. This property may be important in interacting with the bacterial cell membrane. In addition, the two monomers enclose free space between them in form of a tunnel that is also open towards the apolar side. Alternatively, this arrangement allows the antibiotic dimer to join its substrate and anchor it to the cell membrane with its long fatty acid sidechains.

The binding cleft is constituted by mostly hydrophobic amino acids, although the $\mathrm{Ca}^{2+}$-ions in intermolecular connection points are located in a highly polar and charged 
environment. The distance between the $\mathrm{Ca}^{2+}$-ions stabilising the dimer is about $9.2 \AA$, which corresponds well to the length of the repeating unit in lipoteichoic acids, which follows a similar frequency alteration in hydrophobicity: the highly charged phosphate groups are linked via a glycerol that is acylated on the middle OH-group by a D-Ala residue (Figure 5.3). The distance between the oxygen atoms of neighbouring phosphate groups is about $8.4 \AA$ if the chain is in fully extended conformation. The opening on the tsushimycin dimer is exactly where the D-Ala residue would be if the phosphate groups were located at the $\mathrm{Ca}^{2+}$-ions. Binding of tsushimycin to lipoteichoic acids would therefore involve the polymer glycerol phosphate chain to run between tsushimycin monomers, with phosphate groups anchored to $\mathrm{Ca}^{2+}$-ions and D-Ala residues pointing out through the hole on the antibiotic dimer (Figure 5.15). Although both tsushimycin and lipoteichoic acids are polyanionic species, $\mathrm{Ca}^{2+}$-ions present in the interface may provide sufficient positive charge for both of them to bind. Lipotechoic acids are known to interact with divalent cations, and therefore may well correspond to the site of action of lipopeptide antibiotics.

\section{Micelles}

In aqueous solutions lipopeptides are shown to associate to micelles in a $\mathrm{Ca}^{2+}$-dependent manner. In the absence of $\mathrm{Ca}^{2+}$, particles with hydrodynamic radius around $66 \mathrm{~nm}$ were found that correspond to micelle sizes up to several million Da. On the other hand, the $\mathrm{Ca}^{2+}$-salt exhibits in solution a narrow distribution centred at $3.15 \mathrm{~nm}$ and is equivalent to a micelle with $55 \mathrm{kDa}$ size constituted by 30-40 antibiotic molecules. Micelles found in the tsushimycin crystal are significantly smaller than this as they consist of only 12 molecules, but one has to take into consideration that they were grown in highly alcoholic solution that may affect micelle sizes. This structure can therefore also be regarded as a representative of a functional micelle.

\section{Implications for antibacterial action}

The exact mechanism of action of lipopeptide antibiotics has not been unambiguously identified yet, although several possibilities were proposed. Crystal structure investigations were initiated in order to clarify the underlying molecular basis of antimicrobial potency. Although no conclusive decisions can be drawn from the crystal structure, a number of important observations can be made.

Based on the crystal structure, tsushimycin is likely to act as a dimer. Since dimers are associated to expose highly polar surfaces to the solvent, it is unlikely that they penetrate the 
bacterial cell membrane albeit the formation of inverse micelles cannot be excluded. Thus, the periplasmic space can be proposed as their primary site of action, which limits possible mechanisms to:

(a) Inhibition of phospho-N-acetylmuramoyl-pentapeptide-transferase (MraY), which is a transmembrane protein and may allosterically be regulated from the extracellular side of the membrane.

(b) Interaction with lipoteichoic acids, which are on the cell surface and are therefore accessible to the antibiotic.

(c) Dissipation of the membrane potential, but probably not with an ion carrier mechanism.

The toxic effect of these antibiotics was predominantly responsible for the cancellation of clinical trials with daptomycin. Although dosing regimens provide a solution in minimising side effects, reduction of toxicity is an important issue in antibiotic development (Debono et al., 1988). The structure of friulimicins, which are considerably less toxic, would therefore be also of interest in the design of novel lipopeptide antibiotics. The recent availability of the crystal structure of tsushimycin may provide a quick way of overcoming the phase problem and determine the structure of friulimicin as well. 



\section{SUMMARY}

Peptide antibiotics belonging to three different families were investigated using X-ray crystallography. The peptaibol antibiotics cephaibol A, B, C, D and E differ only marginally in chemical structure, although this is associated with a variation more than an order of magnitude in the antibacterial potency. No diffraction quality crystals were obtained from cephaibol D and cephaibol $\mathrm{E}$ and therefore only unit cell dimensions were established. Cephaibol A, B and C gave well-diffracting crystals and their structures were determined at ca. $0.9 \AA$ resolution. They adopt a helical conformation with a sharp bend at the central hydroxyproline. Superposition of the models shows that the N-terminal helix is rigid and the C-terminus is flexible, but the differences in three-dimensional structure are negligible. However, there are differences in the hydrogen-bonding pattern for the three structures, and only in the case of cephaibol $\mathrm{C}$ does the packing emulate the formation of a membrane channel believed to be important for their biological function.

Feglymycin is a unique peptide in terms of chemical structure and antimicrobial action. The chirality of amino acids alternates regularly, except for at the termini, where there are deviations from this pattern. Two crystal modifications were obtained: one form grew from aqueous solvent, while the other one from a highly alcoholic environment. In both crystal forms the peptide was found to form asymmetric dimers in form of an antiparallel doublestranded double $\beta^{9.0}$-helix and resemble the membrane channel peptide gramicidin. However, the feglymycin channel is blocked by two phenylalanine sidechains and the dimer is not as long as gramicidin and it is probably too short to span a biological membrane. The dimeric structure is stabilised by numerous hydrogen bonds and therefore it is considerably rigid. Based on the model, it is more likely that feglymycin is a wide-spectrum enzyme inhibitor than a membrane agent.

Friulimicin and tsushimycin belong to the group of lipopeptide antibiotics, and are highly active against multiresistant bacteria. Two crystal forms were obtained from friulimicin, but in one case the crystal diffracted to moderate resolution only, while in the other case it proved to be non-merohedrally twinned and therefore in neither case could the phase problem be solved. Tsushimycin gave one crystal modification and was solved using $a b$ initio direct methods. The structure contains 12 molecules; each of these binds a $\mathrm{Ca}^{2+}$-ion that seems to be important in stabilising the three-dimensional structure. The molecules 
associate to dimers via interactions mediated by additional $\mathrm{Ca}^{2+}$-ions. The crystal structure confirms the sequence assignment proposed by Vértesy et al. (2000). The packing emulates the structure of a micelle and the molecules act as separators between polar and non-polar cavities. The mechanism of antimicrobial action cannot be unambiguously identified from the crystal structure, but it seems likely that the antibiotic cannot enter the bacterial cell and acts on its surface. 


\section{REFERENCES}

Abbanat, D., Macielag, M. \& Bush, K. (2003). Novel antibacterial agents for the treatment of serious Gram-positive infections. Expert Opin. Investig. Drugs 12, 379-399.

Alborn, W. E. Jr., Allen, N. E. \& Preston, D. A. (1991). Daptomycin disrupts membrane potential in growing Staphylococcus aureus. Antimicrob. Agents Chemother. 35, 22822287.

Alkhatib, G., Combadiere, C., Broder, C. C., Feng, Y., Kennedy, P. E., Murphy, P. M. \& Berger, E. A. (1996). CC CKR5: a RANTES, MIP-1 $\alpha$, MIP-1 $\beta$ receptor as a fusion cofactor for macrophage-tropic HIV-1. Science 272, 1955-1958.

Allen, F. H. (2002). The Cambridge Structural Database: a quarter of a million crystal structures and rising. Acta Cryst. B58, 380-388.

Allen, F. H., Kennard, O., Watson, D. G., Brammer, L., Orpen, A. G. \& Taylor, R. (1992). Typical interatomic distances: organic compounds. International Tables for Crystallography, Vol. C, edited by A. J. C. Wilson, pp. 685-706. Kluwer Academic Publishers, Dordrecht.

Allen, N. E., Hobbs, J. N. Jr., Alborn, W. E. Jr. (1987). Inhibition of peptidoglycan biosynthesis in Gram-positive bacteria by LY146032. Antimicrob. Agents Chemother. 31, 1093-1099.

Amidi, S., Solter, S., Rashidian, B., Zokajan, A.-R. \& Razmjolan, F. (1975). Antibiotic use and abuse among physicians in private practice in Shiraz, Iran. Med. Care 13, 341345 .

Anders, R., Ohlenschlager, O., Soskic, V., Wenschuh, H., Heise, B. \& Brown, L. R. (2000). The NMR solution structure of the ion channel peptaibol chrysospermin C bound to dodecylphosphocholine micelles. Eur. J. Biochem. 267, 1784-1794.

Andersson, D. I. (2003). Persistence of antibiotic resistant bacteria. Curr. Opin. Microbiol. 6, 452-456.

Asano, T., Matsuoka, K., Hida, T., Kobayashi, M., Kitamura, Y., Hayakawa, T., Iinuma, S., Kakinuma, A. \& Kato, K. (1994). Novel retrovirus protease inhibitors, RPI-856 A, B, C and D, produced by Streptomyces sp. AL-322. J. Antibiot. 47, 557-565.

Balaram, P., Krishna, K., Sukumar, M., Mellor, I. R. \& Sansom, M. S. (1992). The properties of ion channels formed by zervamicins. Eur. Biophys. J. 21, 117-128.

Balashova, T. A., Shenkarev, Z. O., Tagaev, A. A., Ovchinnikova, T. V., Raap, J. \& Arseniev, A. S. (2000). NMR structure of the channel-former zervamicin IIb in isotropic solvents. FEBS Lett. 466, 333-336. 
Balint, G. A. (2001). Antiretroviral therapeutic possibilities for human immunodeficiency virus/acquired immunodeficiency syndrome. Pharmacol. Ther. 89, 17-27.

Banerjee, D. K. (1989). Amphomycin inhibits mannosylphosphoryldolichol synthesis by forming a complex with dolichylmonophosphate. J. Biol. Chem. 264, 2024-2028.

Baranowski, E., Ruiz-Jarabo, C. M. \& Domingo, E. (2001). Evolution of cell recognition by viruses. Science 292, 1102-1105.

Barna, J. C. J. \& Williams, D. H. (1984). The structure and mode of action of glycopeptide antibiotics of the vancomycin group. Annu. Rev. Microbiol. 38, 339-357.

Barry, A. L., Fuchs, P. C. \& Brown, S. D. (2001). In vitro activities of daptomycin against 2,789 clinical isolates from 11 North American medical centers. Antimicrob. Agents Chemother. 45, 1919-1922.

Beach, J. W. (1998). Chemotherapeutic agents for human immunodeficiency virus infection: mechanism of action, pharmacokinetics, metabolism, and adverse reactions. Clin. Ther. 20, 2-25.

Bengmark, S. (2002). Gut microbial ecology in critical illness: is there a role for prebiotics, probiotics, and synbiotics? Curr. Opin. Crit. Care 8, 145-151.

Berger, E. A. (1997). HIV entry and tropism: the chemokine receptor connection. AIDS 11, A3-A16.

Bodanszky, M., Chaturvedi, N. C., Scozzie, J. A., Griffith, R. K. \& Bodanszky, A. (1969). Constituents of amphomycin. Antimicrob. Agents Chemother. 9, 135-138.

Bodanszky, M., Sigler, G. F. \& Bodanszky, A. (1973). Structure of the peptide antibiotic amphomycin. J. Am. Chem. Soc. 95, 2352-2357.

Boyd, M. R., Hallock, Y. F., Cardellina, J. H. 2nd., Manfredi, K. P., Blunt, J. W., McMahon, J. B., Buckheit, R. W. Jr., Bringmann, G., Schaffer, M., Cragg, G. M., Thomas, D. W. \& Jato, J. G. (1994). Anti-HIV michellamines from Ancistrocladus korupensis. J. Med. Chem. 37, 1740-1745.

Bronson, J. J. \& Barrett, J. F. (2001). Quinolone, everninomycin, glycylcycline, carbapenem, lipopetide and cephem antibacterials in clinical development. Curr. Med. Chem. 8, 1775-1793.

Bruker Nonius (2002). Cell_now, Proteum, RLATT, SAINT, SADABS, SMART, TWINABS, XPREP and XP computer programs.

Brünger, A. T. (1992). Free R value: a novel statistical quantity for assessing the accuracy of crystal structures. Nature 355, 472-475.

Bugg, T. D. H. \& Walsh, C. T. (1992). Intracellular steps of bacterial cell wall peptidoglycan biosynthesis: antibiotics, and antibiotic resistance. Nat. Prod. Rep. 9, 199-215. 
Burkhart, B. M., Gassman, R. M., Langs, D. A., Pangborn, W. A. \& Duax, W. L. (1998). Heterodimer formation and crystal nucleation of gramicidin D. Biophys. J. 75, 2135 2146.

Burkhart, B. M., Li, N., Langs, D. A., Pangborn, W. A. \& Duax, W. L. (1998). The conducting form of gramicidin A is a right-handed double-stranded double helix. Proc. Natl. Acad. Sci. USA 95, 12950-12955.

Burkhart, B. M., Gassman, R. M., Langs, D. A., Pangborn, W. A., Duax, W. L. \& Pletnev, V. (1999). Gramicidin D conformation, dynamics and membrane ion transport. Biopolymers 51, 129-144.

Canepari, P., Boaretti, M., Del Mar LLeó, M. \& Satta, G. (1990). Lipoteichoic acid as a new target for activity of antibiotics: mode of action of daptomycin (LY146032). Antimicrob. Agents Chemother. 34, 1220-1226.

Chan, D. C., Fass, D., Berger, J. M. \& Kim, P. S. (1997). Core structure of gp41 from the HIV envelope glycoprotein. Cell 89, 263-273.

Chatterjee, A. N. \& Perkins, H. R. (1966). Compounds formed between nucleotides related to the biosynthesis of bacterial cell wall and vancomycin. Biochem. Biophys. Res. Commun. 24, 489-494.

Chugh, J. K., Brückner, H. \& Wallace, B. A. (2002). Model for a helical bundle channel based on the high-resolution crystal structure of trichotoxin_A50E. Biochemistry 41, 12934-12941.

Coates, M. E. (1975). The influence of the gut microflora on the nutrition of its host. Bibl. Nutr. Dieta 22, 101-108.

Cochran, W. (1955). Relations between the phases of structure factors. Acta Cryst. 8, 473478.

Cocito, C., Di Giambattista, M., Nyssen, E. \& Vannuffel, P. (1997). Inhibition of protein synthesis by streptogramins and related antibiotics. J. Antimicrob. Chemother. 39, A7A13.

Colonna-Cesari, F., Premilat, S., Heitz, F., Spach, G. \& Lotz, B. (1977). Helical structures of poly(D-L-peptides). A conformational energy analysis. Macromolecules 10, 12841288.

Cruickshank, D. W. J. (1970). Least-squares refinement of atomic parameters. Crystallographic computing, edited by F. R. Ahmed, S. R. Hall \& C. P. Huber, pp. 187196. Munksgaard, Copenhagen.

Cruickshank, D. W. J. (1999). Remarks about protein structure precision. Acta Cryst. D55, 583-601.

Cullen, B. R. (1995). Regulation of HIV gene expression. AIDS 9, A19-A32. 
Cundliffe, E. (1984). Self defence in antibiotic-producing organisms. Br. Med. Bull. 40, 61-67.

Davies, W. L., Grunert, R. R., Haff, R. F., McGahen, J. W., Neumayer, E. M., Paulshock, M., Watts, J. C., Wood, T. R., Hermann, E. C. \& Hoffmann, C. E. (1964). Antiviral activity of 1-adamantanamine (amantadine). Science 144, 862-863.

Debono, M., Abbot, B. J., Molloy, R. M., Fukuda, D. S., Hunt, A. H., Daupert, V. M., Counter, F. T., Ott, J. L., Carrell, C. B., Howard, L. C., Boeck, L. D. \& Hamill, R. L. (1988). Enzymatic and chemical modifications of lipopeptide antibiotic A21978C: the synthesis and evaluation of daptomycin (LY146032). J. Antibiot. 41, 1093-1105.

Degenkolb, T., Berg, A., Gams, W., Schlegel, B. \& Gräfe, U. (2003). The occurrence of peptaibols and structurally related peptaibiotics in fungi and their mass spectrometric identification via diagnostic fragment ions. J. Peptide Sci. 9, 666-678.

DeLano, W. L. (2003). The PyMOL molecular graphics system. DeLano Scientific LLC, San Carlos, CA, USA.

Dersch, P. (2003). Molecular and cellular mechanisms of bacterial entry into host cells. Contrib. Microbiol. 10, 183-209.

Diamond, R. (1969). Profile analysis in single crystal diffractometry. Acta Cryst. A25, 43-55.

Di Giambattista, M., Chinali, G. \& Cocito, C. (1989). The molecular basis of the inhibitory activities of type A and type B synergimycins and related antibiotics on ribosomes. J. Antimicrob. Chemother. 24, 485-507.

Di Guilmi, A. M., Dessen, A., Dideberg, O. \& Vernet, T. (2002). Bifunctional penicillinbinding proteins: focus on the glycosyltransferase domain and its specific inhibitor moenomycin. Curr. Pharm. Biotechnol. 3, 63-75.

Dragic, T., Litwin, V., Allaway, G. P., Martin, S. R., Huang, Y., Nagashima, K. A., Cayanan, C., Maddon, P. J., Koup, R. A., Moore, J. P. \& Paxton, W. A. (1996). HIV-1 entry into CD4+ cells is mediated by the chemokine receptor CC-CKR-5. Nature 381, 667-673.

Duax, W. L., Griffin, J. F., Langs, D. A., Smith, G. D., Grochulski, P., Pletnev, V. \& Ivanov, V. (1996). Molecular structure and mechanisms of action of cyclic and linear ion transport antibiotics. Biopolymers 40, 141-155.

Duclohier, H., Snook, C. F. \& Wallace, B. A. (1998). Antiamoebin can function as a carrier or as a pore-forming peptaibol. Biochim. Biophys. Acta 1415, 255-260.

Duisenberg, A. J. M. (1992). Indexing in single-crystal diffractometry with an obstinate list of reflections. J. Appl. Cryst. 25, 92-96.

Duisenberg, A. J. M., Kroon-Batenburg, L. M. J. \& Schreurs, A. M. M. (2003). An intensity evaluation method: EVAL-14. J. Appl. Cryst. 36, 220-229. 
Durckheimer, W. (1975). Tetracyclines: chemistry, biochemistry, and structure-activity relations. Angew. Chem. Int. Ed. Engl. 14, 721-734.

El Mashak, E. M. \& Tocanne, J. F. (1980). Polymyxin B-phosphatidylglycerol interactions. A monolayer $(\pi, \Delta \mathrm{V})$ study. Biochim. Biophys. Acta 596, 165-179.

Emmerson, A. M. \& Jones, A. M. (2003). The quinolones: decades of development and use. J. Antimicrob. Chemother. 51, S13-S20.

Engh, R. A. \& Huber, R. (1991). Accurate bond and angle parameters for X-ray protein structure refinement. Acta Cryst. A47, 392-400.

Esnouf, R. M. (1999). Further additions to Molscript version 1.4, including reading and contouring of electron-density maps. Acta Cryst. D55, 938-940.

Fox, R. O. \& Richards, F. M. (1982). A voltage-gated ion channel model inferred from the crystal structure of alamethicin at 1.5-Å resolution. Nature 300, 325-330.

Frank, I. (2002). Antivirals against HIV-1. Clin. Lab. Med. 22, 741-757.

Frankel, A. D. \& Young, J. A. (1998). HIV-1: fifteen proteins and an RNA. Annu. Rev. Biochem. 67, 1-25.

Frère, J. M. \& Joris, B. (1985). Penicillin-sensitive enzymes in peptidoglycan biosynthesis. Crit. Rev. Microbiol. 11, 299-396.

Fujinaga, M. \& Read, R. J. (1987). Experiences with a new translation-function program. J. Appl. Cryst. 20, 517-521.

Fujino, M. (1965). On glumamycin, a new antibiotic. VI. An approach to the amino acid sequence. Bull. Chem. Soc. Jpn. 38, 517-522.

Furesz, S. (1970). Chemical and biological properties of rifampicin. Antibiot. Chemother. 16, 316-351.

Galbraith, T. P., Harris, R., Driscoll, P. C. \& Wallace, B. A. (2003). Solution NMR studies of antiamoebin, a membrane channel-forming polypeptide. Biophys. J. 84, 185-194.

Gale, E. F., Cundliffe, E., Reynolds, P. E., Richmond, M. H. \& Waring, M. J. (1981). The molecular basis of antibiotic action, 2. ed. Wiley-Interscience, New York.

Garrison, M. W., Rotschafer, J. C. \& Crossley, K. B. (1989). Suboptimal effect of daptomycin in the treatment of bacteremias. South Med. J. 82, 1414-1415.

Geiger, T. \& Clarke, S. (1987). Deamidation, isomeration, and racemization at asparaginyl and aspartyl residues in peptides. J. Biol. Chem. 262, 785-794.

Gorbach, S. L. (1997). Treating diarrhoea. BMJ 314, 1776-1777.

Gordon, L. G. \& Haydon, D. A. (1972). The unit conductance channel of alamethicin. Biochim. Biophys. Acta 255, 1014-1018. 
Gracey, M. S. (1981). Nutrition, bacteria and the gut. Br. Med. Bull. 37, 71-75.

Gulick, R. M. (2003). New antiretroviral drugs. Clin. Microbiol. Infect. 9, 186-193.

Hanke, W. \& Boheim, G. (1980). The lowest conductance state of the alamethicin pore. Biochim. Biophys. Acta 596, 456-462.

Hauck, C. R. (2002). Cell adhesion receptors - signaling capacity and exploitation by bacterial pathogens. Med. Microbiol. Immunol. 191, 55-62.

Hayes, J. D. \& Wolf, C. R. (1990). Molecular mechanisms of drug resistance. Biochem. J. 272, 281-295.

Heinemann, B., Kaplan, M. A., Muir, R. D. \& Hooper, I. R. (1953). Amphomycin, a new antibiotic. Antibiot. Chemother. 3, 1239-1242.

Hermsen, E. D., Sullivan, C. J. \& Rotschafer, J. C. (2003). Polymyxins: pharmacology, pharmacokinetics, pharmacodynamics, and clinical applications. Infect. Dis. Clin. North Am. 17, 545-562.

Hooper, L. V., Midtvedt, T. \& Gordon, J. I. (2002). How host-microbial interactions shape the nutrient environment of the mammalian intestine. Annu. Rev. Nutr. 22, 283-307.

Inoue, M. (1962a). On glumamaycin, a new antibiotic. II. Isolation and identification of amino acids constituting glumamycin. Bull. Chem. Soc. Jpn. 35, 1249-1254.

Inoue, M. (1962b). On glumamaycin, a new antibiotic. III. Fatty acid, a constituent of the antibiotic. Bull. Chem. Soc. Jpn. 35, 1255-1257.

Inoue, M. (1962c). On glumamaycin, a new antibiotic. IV. The amino acid moiety. Bull. Chem. Soc. Jpn. 35, 1556-1559.

Jacobo-Molina, A., Ding, J., Nanni, R. G., Clark, A. D. Jr., Lu, X., Tantillo, C., Williams, R. L., Kamer, G., Ferris, A. L., Clark, P., Hizi, A., Hughes, S. H. \& Arnold, E. (1993). Crystal structure of human immunodeficiency virus type 1 reverse transcriptase complexed with double-stranded DNA at 3.0 $\AA$ resolution shows bent DNA. Proc. Natl. Acad. Sci. USA 90, 6320-6324.

Jancarik, J. \& Kim, S. H. (1991). Sparse matrix sampling: a screening method for crystallization of proteins. J. Appl. Cryst. 24, 409-411.

Kabsch, W. (1976). A solution for the best rotation to relate two sets of vectors. Acta Cryst. A32, 922-923.

Kabsch, W. (1993). Automatic processing of rotation diffraction data from crystals of initially unknown symmetry and cell constants. J. Appl. Cryst. 26, 795-800.

Kabsch, W. (2001). Integration, scaling, space-group assignment and post refinement. International Tables for Crystallography, Vol. F, edited by E. Arnold \& M. G. Rossmann, pp. 218-225. Kluwer Academic Publishers, Dordrecht. 
Kamiryo, T. \& Matsuhashi, M. (1972). The biosynthesis of the cross-linking peptides in the cell wall peptidoglycan of Staphylococcus aureus. J. Biol. Chem. 247, 6306-6311.

Kaneko, I., Kamoshida, K. \& Takahashi, S. (1989). Complestatin, a potent anticomplement substance produced by Streptomyces lavendulae. I. Fermentation, isolation and biological characterization. J. Antibiot. 42, 236-241.

Kang, M. S., Spencer, J. P. \& Elbein, A. D. (1978). Amphomycin inhibition of mannose and GlcNAc incorporation into lipid-linked saccharides. J. Biol. Chem. 253, 8860-8866.

Karle, I. L., Flippen-Anderson, J. L., Agarwalla, S. \& Balaram, P. (1991). Crystal structure of [Leu1]zervamicin, a membrane ion-channel peptide: Implications for gating mechanisms. Proc. Natl. Acad. Sci. USA 88, 5307-5311.

Karle, I. L., Perozzo, M. A., Mishra, V. K. \& Balaram, P. (1998). Crystal structure of the channel-forming polypeptide antiamoebin in a membrane-mimetic enviroment. Proc. Natl. Acad. Sci. USA 95, 5501-5504.

Karle, J. \& Hauptman, H. (1956). A theory of phase determination for the four types of non-centrosymmetric space groups 1P222, 2P22, 3P $12,3 \mathrm{P}_{2} 2$. Acta Cryst. 9, 635-651.

Katz, R. A. \& Skalka, A. M. (1994). The retroviral enzymes. Annu. Rev. Biochem. 63, 133173.

Kayser, F. H. (2003). Safety aspects of enterococci from the medical point of view. Int. J. Food Microbiol. 88, 255-262.

Ketchem, R. R., Lee, K. C., Huo, S. \& Cross, T. A. (1996). Macromolecular structural elucidation with solid-state NMR-derived orientational constraints. J. Biomol. NMR 8, $1-14$.

Kissinger, C. R., Gehlhaar, D. K. \& Fogel, D. B. (1999). Rapid automated molecular replacement by evolutionary search. Acta Cryst. D55, 484-491.

Kitchen, V. S., Skinner, C., Ariyoshi, K., Lane, E. A., Duncan, I. B., Burckhardt, J., Burger, H. U., Bragman, K., Pinching, A. J. \& Weber, J. N. (1995). Safety and activity of saquinavir in HIV infection. Lancet 345, 952-955.

Klare, I., Konstabel, C., Badstübner, D., Werner, G. \& Witte, W. (2003). Occurence and spread of antibiotic resistances in Enterococcus faecium. Int. J. Food Microbiol. 88, 269290.

Klemm, P. \& Schembri, M. A. (2000). Bacterial adhesins: function and structure. Int. J. Med. Microbiol. 290, 27-35.

Kong, F. \& Carter, G. T. (2003). Structure determination of glycinocins A to D, further evidence for the cyclic structure of the amphomycin antibiotics. J. Antibiot. 56, 557-564.

Kotra, L. P., Haddad, J. \& Mobashery, S. (2000). Aminoglycosides: perspectives on mechanisms of action and resistance and strategies to counter resistance. Antimicrob. Agents Chemother. 44, 3249-3256. 
Kraulis, P. J. (1991). MOLSCRIPT: a program to produce both detailed and schematic plots of protein structures. J. Appl. Cryst. 24, 946-950.

Kronen, M., Görls, H., Nguyen, H.-H., Reissmann, S., Bohl, M., Sühnel, J. \& Gräfe, U. (2003). Crystal structure and conformational analysis of ampullosporin A. J. Peptide Sci. 9, 729-744.

Kwong, P. D., Wyatt, R., Robinson, J., Sweet, R. W., Sodroski, J. \& Hendrickson, W. A. (1998). Structure of an HIV gp120 envelope glycoprotein in complex with the CD4 receptor and a neutralizing human antibody. Nature 393, 648-659.

Lambert, P. A., Hancock, I. C. \& Baddiley, J. (1977). Occurence and function of membrane teichoic acids. Biochim. Biophys. Acta 472, 1-12.

Langs, D. A. (1988). Three-dimensional structure at $0.86 \AA$ of the uncomplexed form of the transmembrane ion channel peptide gramicidin A. Science 241, 188-191.

Larder, B. A. (1995). Viral resistance and the selection of antiretroviral combinations. J. Acquir. Immune Defic. Syndr. Hum. Retrovirol. 10, S228-S233.

Latorre, R. \& Alvarez, O. (1981). Voltage-dependent channels in planar lipid bilayer membranes. Physiol. Rev. 61, 77-150.

Lebek, G. \& Cottier, H. (1992). Notes on the bacterial content of the gut. Curr. Stud. Hematol. Blood Transfus. 59, 1-18.

Leclerc, G., Rebuffat, S., Goulard, C. \& Bodo, B. (1998). Directed biosynthesis of peptaibol antibiotics in two Trichoderma strains. I. Fermentation and isolation. J. Antibiot. 51, 170-183.

Lehmann, M. S. \& Larsen, F. K. (1979). A method for location of the peaks in step-scan measured Bragg reflexions. Acta Cryst. A30, 580-584.

Leslie, A. G. W. (1999). Integration of macromolecular diffraction data. Acta Cryst. D55, 1696-1702.

Levy, S. B. (1992). The antibiotic paradox: how miracle drugs are destroying the miracle. Plenum Publishing, New York.

Levy, S. B. (1998). The challenge of antibiotic resistance. Scientific American 278, 46-53.

Levy, S. B. (2001). Antibiotic resistance: consequences of inaction. Clin. Infect. Dis. 33, S124-S129.

Levy, S. B. (2002). Factors impacting on the problem of antibiotic resistance. J. Antibiot. Chemother. 49, 25-30.

Linden, P. K. (2002). Treatment options for vancomycin-resistant enterococcal infections. Drugs 62, 425-441. 
Little, S. J., Daar, E. S., D'Aquila, R. T., Keiser, P. H., Connick, E., Whitcomb, J. M., Hellmann, N. S., Petropoulos, C. J., Sutton, L., Pitt, J. A., Rosenberg, E. S., Koup, R. A., Walker, B. D. \& Richman, D. D. (1999). Reduced antiretroviral susceptibility among patients with primary HIV infection. JAMA 282, 1142-1149.

Lomakina, N. N. \& Brazhnikova, M. G. (1959). The composition of crystallomycin. Biokhimiia 24, 425-431.

Lomize, A. L., Orekhov, V. Yu. \& Arseniev, A. S. (1992). Refinement of the spatial structure of the gramicidin A transmembrane ion-channel. Bioorg. Khim. 18, 182-200.

Mansky, L. M. \& Temin, H. M. (1995). Lower in vivo mutation rate of human immunodeficiency virus type 1 than that predicted from the fidelity of purified reverse transcriptase. J. Virol. 69, 5087-5094.

Marra, A. \& Isberg, R. R. (1996). Common entry mechanisms. Bacterial pathogenesis. Curr. Biol. 6, 1084-1086.

Matthée, G., Wright, A. D. \& König, G. M. (1999). HIV reverse transcriptase inhibitors of natural origin. Planta Med. 65, 493-506.

McMahon, J. B., Currens, M. J., Gulakowski, R. J., Buckheit, R. W. Jr., LackmanSmith, C., Hallock, Y. F. \& Boyd, M. R. (1995). Michellamine B, a novel plant alkaloid, inhibits human immunodeficiency virus-induced cell killing by at least two distinct mechanisms. Antimicrob. Agents Chemother. 39, 484-488.

McRee, D. E. (1999). XtalView - a versatile program for manipulating atomic coordinates and electron density. J. Struct. Biol. 125, 156-165.

Menestrina, G., Voges, K. P., Jung, G. \& Boheim, G. (1986). Voltage-dependent channel formation by rods of helical polypeptides. J. Membrane Biol. 93, 111-132.

Merritt, E. A. \& Bacon, D. J. (1997). Raster3D: photorealistic molecular graphics. Methods Enzymol. 277, 505-524.

Metges C. C. (2000). Contribution of microbial amino acids to amino acid homeostasis of the host. J. Nutr. 130, 1857S-1864S.

Milatovic, D. \& Braveny, I. (1987). Development of resistance during antibiotic therapy. Eur. J. Clin. Microbiol. 6, 234-244.

Miller, Y. W., Eady, E. A., Lacey, R. W., Cove, J. H., Joanes, D. N. \& Cunliffe, W. J. (1996). Sequential antibiotic therapy for acne promotes the carriage of resistant staphylococci on the skin of contacts. J. Antimicrob. Chemother. 38, 829-837.

Milov, A. D., Tsvetkov, Yu. D., Formaggio, F., Crisma, M., Toniolo, C. \& Raap, J. (2003). Self-assembling and membrane modifying properties of a lipopeptaibol studied by CW-ESR and PELDOR spectroscopies. J. Peptide Sci. 9, 690-700. 
Moews, P. C. \& Kretsinger, R. H. (1975). Refinement of the structure of carp muscle calcium-binding parvalbumin by model building and difference Fourier analysis. J. Mol. Biol. 91, 201-225.

Moulard, M., Lortat-Jacob, H., Mondor, I., Roca, G., Wyatt, R., Sodroski, J., Zhao, L., Olson, W., Kwong, P. D. \& Sattentau, Q. J. (2000). Selective interactions of polyanions with basic surfaces on human immunodeficiency virus type $1 \mathrm{gp} 120$. J. Virol. 74, 1948-1960.

Naganawa, H., Hamada, M., Maeda, K., Okami, Y., Takeuchi, T. \& Umezawa, H. (1968). Laspartomycin, a new antistaphylococcal peptide. J. Antibiot. 21, 55-62.

Nagy, K., Young, M., Baboonian, C., Merson, J., Whittle, P. \& Oroszlan, S. (1994). Antiviral activity of human immunodeficiency virus type 1 protease inhibitors in a single cycle of infection: evidence for a role of protease in the early phase. J. Virol. 68, 757-765.

Nelson, M. L. \& Levy, S. B. (1999). Reversal of tetracycline resistance mediated by different bacterial tetracycline resistance determinants by an inhibitor of the Tet(B) antiport protein. Antimicrob. Agents Chemother. 43, 1719-1724.

Neu, H. C. (1992). The crisis in antibiotic resistance. Science 257, 1064-1073.

Neuhaus, F. C. \& Hammes, W. P. (1981). Inhibition of cell wall biosynthesis by analogues and alanine. Pharmacol. Ther. 14, 265-319.

Ng, T. B., Huang, B., Fong, W. P. \& Yeung, H. W. (1997). Anti-human immunodeficiency virus (anti-HIV) natural products with special emphasis on HIV reverse transcriptase inhibitors. Life Sci. 61, 933-949.

Noller, H. F. (1991). Ribosomal RNA and translation. Annu. Rev. Biochem. 60, 191-227.

Norrby, R. (2001). Linezolid - a review of the first oxazolidinone. Expert Opin. Pharmacother. 2, 293-302.

Ochoa, T. J. \& Cleary, T. G. (2003). Epidemology and spectrum of disease of Escherichia coli O157. Curr. Opin. Infect. Dis. 16, 259-263.

Omobosola, A. \& Henry, K. (2003). Current trends in the treatment of HIV infection, 2003. Minn. Med. 86, 39-44.

Otwinowski, Z. \& Minor, W. (1997). Processing of X-ray diffraction data collected in oscillation mode. Methods Enzymol. 276, 307-326.

Pani, A., Loi, A. G., Mura, M., Marceddu, T., La Colla, P. \& Marongiu, M. E. (2002). Targeting HIV: old and new players. Curr. Drug Targets Infect. Dis. 2, 17-32.

Pannu, N. S. \& Read, R. J. (1996). Improved refinement through maximum likelihood. Acta Cryst. A52, 659-668. 
Paterson, D. L., Swindells, S., Mohr, J., Brester, M., Vergis, E. M., Squier, C., Wagener, M. M. \& Singh, N. (2000). Adherence to protease inhibitor therapy and outcomes in patients with HIV infection. Ann. Intern. Med. 133, 21-30.

Peggion, C., Formaggio, F., Crisma, M., Epand, R. F., Epand, R. M. \& Toniolo, C. (2003). Trichogin: a paradigm for lipopeptaibols. J. Peptide Sci. 9, 679-689.

Pluymers, W., De Clercq, E. \& Debyser, Z. (2001). HIV-1 integration as a target for antiretroviral therapy: a review. Curr. Drug Targets Infect. Dis. 1, 133-149.

Read, R. J. (1990). Structure-factor probabilities for related structures. Acta Cryst. A46, 900912.

Rehm, S. J. (2002). Two new treatment options for infections due to drug-resistant Grampositive cocci. Cleve. Clin. J. Med. 69, 397-401.

Reynolds, P. E. (1989). Structure, biochemistry and mechanism of action of glycopeptide antibiotics. Eur. J. Clin. Microbiol. Infect. Dis. 8, 943-950.

Ritzau, M., Heinze, S., Dornberger, K., Berg, A., Fleck, W., Schlegel, B., Härtl, A. \& Gräfe, U. (1997). Ampullosporin, a new peptaibol-type antibiotic from Sepedonium ampullosporum HKI-0053 with neuroleptic activity in mice. J. Antibiot. 50, 722-728.

Rizzuto, C. D., Wyatt, R., Hernandez-Ramos, N., Sun, Y., Kwong, P. D., Hendrickson, W. A. \& Sodroski, J. (1998). A conserved HIV gp120 glycoprotein structure involved in chemokine receptor binding. Science 280, 1949-1953.

Rossmann, M. G. \& Arnold, E. (1993). Patterson and molecular-replacement techniques. International Tables for Crystallography, Vol. B, edited by U. Shmueli, pp. 235-263. Kluwer Academic Publishers, Dordrecht.

Rossmann, M. G. \& van Beek, C. G. (1999). Data processing. Acta Cryst. D55, 1631-1640.

Rotschafer, J. C., Garrison, M. W. \& Rodvold, K. A. (1988). Therapeutic update on glycopeptide and lipopeptide antibiotics. Pharmacotherapy 8, 211-219.

Russel, A. D. (2003). Biocide use and antibiotic resistance: the relevance of laboratory findings to clinical and environmental situations. Lancet Infect. Dis. 3, 794-803.

Sarges, R. \& Witkop, B. (1956). Gramicidin A. V. The structure of valine- and isoleucinegramicidin A. J. Am. Chem. Soc. 87, 2011-2020.

Schaberg, D. R., Rubens, C. E., Alford, R. H., Farrar, W. E., Schaffner W. \& McGee, Z. A. (1981). Evolution of antimicrobial resistance and nosomical infection. Lessons from the Vanderbilt experience. Am. J. Med. 70, 445-448.

Schiell, M., Hofmann, J., Kurz, M., Schmidt, F. R., Vértesy, L., Vogel, M., Wink, J. \& Seibert, G. (2001). Cephaibols, new peptaibol antibiotics with anthelmintic properties from Acremonium tubaki DSM 12774. J. Antibiot. 54, 220-233. 
Schlegel, B., Härtl, A., Berg, A., Kronen, M., Gräfe, U., Vértesy, L., Wink, J. \& Brückner, H. (2002). Peptaibols as inducers of fungal pigment formation and correlation to hypothermia in mice. Poster No. 15 at the workshop Peptaibols: biosynthesis, structural diversity, bioactivity and mode of action. 9-11 October 2002, Jena, Germany.

Schleifer, K. H. \& Kandler, O. (1972). Peptidoglycan types of bacterial cell walls and their taxonomic implications. Bacteriol. Rev. 36, 407-477.

Schneider, T. R. (2002). A genetic algorithm for the identification of conformationally invariant regions in protein molecules. Acta Cryst. D58, 195-208.

Sebastian, J. \& Faruki, H. (2004). Update on HIV resistance and resistance testing. Med. Res. Rev. 24, 115-125.

Seydel, J. K. (1968). Sulfonamides, structure-activity relationship, and mode of action. Structural problems of the antibacterial action of 4 -aminobenzoic acid (PABA) antagonists. J. Pharm. Sci. 57, 1455-1478.

Shay, A. J., Adam, J., Martin, J. H., Hausmann, W. K., Shu, P. \& Bohonos, N. (1960). Aspartocin. I. Production, isolation, and characteristics. Antibiot. Annu. 7, 194-198.

Sheldrick, G. M. (2002). Macromolecular phasing with SHELXE. Z. Kristallogr. 217, 644650 .

Sheldrick, G. M. \& Schneider, T. R. (1997). SHELXL: high resolution refinement. Methods Enzymol. 277, 319-343.

Sheldrick, G. M., Hauptman, H. A., Weeks, C. M., Miller, M. \& Usón, I. (2001). Ab initio phasing. International Tables for Crystallography, Vol. F, edited by E. Arnold \& M. G. Rossmann, pp. 333-351. Kluwer Academic Publishers, Dordrecht.

Shenkarev, Z. O., Balashova, T. A., Efremov, R. G., Yakimenko, Z. A., Ovchinnikova, T. V., Raap, J. \& Arseniev, A. S. (2002). Spatial structure of zervamicin IIb bound to DPC micelles: implications for voltage-gating. Biophys. J. 82, 762-771.

Shibata, M., Kanzaki, T., Nakazawa, K., Inoue, M., Hitomi, H., Mizuno, K., Fujino, M. \& Miyake, A. (1962). On glumamycin, a new antibiotic. J. Antibiot. 15, 1-6.

Shoji, J. \& Otsuka, H. (1969). Studies on tsushimycin. II. The structures of constituent fatty acids. J. Antibiot. 22, 473-479.

Shoji, J., Kozuki, S., Okamoto, S., Sakazaki, R. \& Otsuka, H. (1968). Studies on tsushimycin. Isolation and characterization of an acidic acylpeptide containing a new fatty acid. J. Antibiot. 21, 439-443.

Smith, H. (1995). The revival of interest in mechanisms of bacterial pathogenicity. Biol. Rev. 70, 277-316.

Snook, C. F., Woolley, G. A., Oliva, G., Pattabhi, V., Wood, S. P., Blundell, T. L. \& Wallace, B. A. (1998). The structure and function of antiamoebin I, a proline-rich membrane-active polypeptide. Structure 6, 783-792. 
Sparks, R. A. (1976). Trends in minicomputer hardware and software. Part I. Crystallographic computing techniques, edited by F. R. Ahmed, K. Huml \& B. Sedláček, pp.452-467. Munksgaard, Copenhagen.

Stella, L., Mazzuca, C., Venanzi, M., Palleschi, A., Didone, M., Formaggio, F., Toniolo, C. \& Pispisa, B. (2004). Aggregation and water-membrane partition as major determinants of the activity of the antibiotic peptide trichogin GA IV. Biophys. J. 86, 936-945.

Steller, I., Bolotovsky, R. \& Rossmann, M. G. (1997). An algorithm for automatic indexing of oscillation images using Fourier analysis. J. Appl. Cryst. 30, 1036-1040.

Strahilevitz, J. \& Rubinstein, E. (2002). Novel agents for resistant Gram-positive infections - a review. Int. J. Infect. Dis. 6, S38-S46.

Stratov, I., DeRose, R., Purcell, D. F. \& Kent, S. J. (2004). Vaccines and vaccine strategies against HIV. Curr. Drug Targets 5, 71-88.

Tally, F. P., Zeckel, M., Wasilewski, M. M., Carini, C., Berman, C. L., Drusano, G. L. \& Oleson, F. B. (1999). Daptomycin: a novel agent for Gram-positive infections. Expert Opin. Investig. Drugs 8, 1223-1238.

Tanaka, H., Iwai, Y., Ōiwa, R., Shinohara, S., Shimizu, S., Oka, T. \& Ōmura, S. (1977). Studies on bacterial cell wall inhibitors. II. Inhibition of peptidoglycan synthesis in vivo and in vitro by amphomycin. Biochim. Biophys. Acta 497, 633-640.

Tanaka, H., Ōiwa, R., Matsukura, S. \& Ōmura, S. (1979). Amphomycin inhibits phospho$\mathrm{N}$-acetylmuramyl-pentapeptide translocase in peptidoglycan synthesis of Bacillus. Biochem. Biophys. Res. Commun. 86, 902-908.

Tanaka, H., Ōiwa, R., Matsukura, S., Inokoshi, J. \& Ōmura, S. (1982). Studies on bacterial cell wall inhibitors. X. Properties of phospho-N-acetylmuramoyl-pentapeptidetransferase in peptidoglycan synthesis of Bacillus megaterium and its inhibition by amphomycin. J. Antibiot. 35, 1216-1221.

Tanaka, H., Matsuzaki, K., Nakashima, H., Ogino, T., Matsumoto, A., Ikeda, H., Woodruff, H. B. \& Ōmura, S. (1997). Chloropeptins, new anti-HIV antibiotics inhibiting gp120-CD4 binding from Streptomyces sp. I. Taxonomy, fermentation, isolation, and physico-chemical properties and biological activities. J. Antibiot. 50, 58-65.

Tosteson, D. C., Cook, P., Andreoli, T. \& Tieffenberg, M. (1967). The effect of valinomycin on potassium and sodium permeability of HK and LK sheep red cells. J. Gen. Physiol. 50, 2513-2525.

Townsley, L. E., Tucker, W. A., Sham, S. \& Hinton, J. F. (2001). Structures of gramicidins A, B, and C incorporated into sodium dodecyl sulfate micelles. Biochemistry 40, 1167611686. 
Usón, I., Sheldrick, G. M., de La Fortelle, E., Bricogne, G., Di Marco, S., Priestle, J. P., Grütter, M. G. \& Mittl, P. R. E. (1999). The $1.2 \AA$ crystal structure of hirustasin reveals the intrinsic flexibility of a family of highly disulphide-bridged inhibitors. Structure 7, $55-63$.

Vértesy, L., Aretz, W., Knauf, M., Markus, A., Vogel, M. \& Wink, J. (1999). Feglymycin, a novel inhibitor of the replication of the human immunodeficiency virus. Fermentation, isolation and structure elucidation. J. Antibiot. 52, 374-382.

Vértesy, L., Ehlers, E., Kogler, H., Kurz, M., Meiwes, J., Seibert, G., Vogel, M. \& Hammann, P. (2000). Friulimicins: Novel lipopeptide antibiotics with peptidoglycan synthesis inhibiting activity from Actinoplanes friuliensis sp. nov. J. Antibiot. 53, 816827.

Vlietnick, A. J., De Bruyne, T., Apers, S. \& Pieters, L. A. (1998). Plant-derived leading compounds for chemotherapy of human immunodeficiency virus (HIV) infection. Planta Med. 64, 97-109.

Vogler, K., Studer, R. O., Lanz, P., Lergier, W. \& Böhni, E. (1965). Synthesen in der

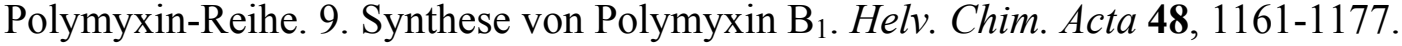

Waxman, D. J. \& Strominger, J. L. (1983). Penicillin-binding proteins and the mechanism of action of beta-lactam antibiotics. Annu. Rev. Biochem. 52, 825-869.

Wegener, H. C., Aarestrup, F. M., Jensen, L. B., Hammerum, A. M. \& Bager, F. (1999). Use of antimicrobial growth promoters in food animals and Enterococcus faecium resistance to therapeutic antimicrobial drugs in Europe. Emerg. Infect. Dis. 5, 329-335.

Williams, D. H., Stone, M. J., Hauck, P. R. \& Rahman, S. K. (1989). Why are secondary metabolites (natural products) biosynthesized? J. Nat. Prod. 52, 1189-1208.

Witte, W., Tschäpe, H., Klare, I. \& Werner, G. (2000). Antibiotics in animal feed. Acta Vet. Scand. Suppl. 93, 37-44.

Wlodawer, A. (1994). Rational drug design: the proteinase inhibitors. Pharmacotherapy 14, S9-S20.

Woodford, N. (2003). Novel agents for the treatment of resistant Gram-positive infections. Expert Opin. Investig. Drugs 12, 117-137.

Wyatt, R. \& Sodroski, J. (1998). The HIV-1 envelope glycoproteins: fusogens, antigens, and immunogens. Science 280, 1884-1888.

Yin, N., Marshall, R. L., Matheson, S. \& Savage, P. B. (2003). Synthesis of lipid A derivatives and their interactions with polymyxin $\mathrm{B}$ and polymyxin $\mathrm{B}$ nonapeptide. J. Am. Chem. Soc. 125, 2426-2435.

Yunis, A. A. (1988). Chloramphenicol: relation of structure to activity and toxicity. Annu. Rev. Pharmacol. Toxicol. 28, 83-100. 


\section{Publikationen}

\section{VERÖFFENTLICHUNGEN IN FACHZEITSCHRIFTEN}

[1] Neculai, A.-M., Cummins, C. C., Neculai, D., Roesky, H. W., Bunkóczi, G., Walfort, B. \& Stalke, D. (2003). Elucidation of a Sc(I) complex by DFT calculations and reactivity studies. Inorg. Chem. 42, 8803-8810.

[2] Bunkóczi, G., Schiell, M., Vértesy, L. \& Sheldrick, G. M. (2003). Crystal structures of cephaibols. J. Peptide Sci. 9, 745-752.

[3] Lehmann, C., Debreczeni, J. É., Bunkóczi, G., Dauter, M., Dauter, Z., Vértesy, L. \& Sheldrick, G. M. (2003). Structures of four crystal forms of decaplanin. Helv. Chim. Acta 86, 1478-1487.

[4] Fitjer, L., Gerke, R., Weiser, J., Bunkóczi, G. \& Debreczeni, J. É. (2003). Helical primary structures of four-membered rings: (M)-trispiro[3.0.0.3.2.2]tridecane. Tetrahedron 59, 4443-4449.

[5] Mernyák, E., Wölfling, J., Bunkóczi, G., Luo, L., Schneider, T. R. \& Schneider, Gy. (2003). Stereoselective synthesis of the two trans-(16-hydroxymethyl)-3-methoxy-13 $\alpha$ estra-1,3,5(10)-trien-17-ol isomers. Coll. Czech. Chem. Commun. 68, 1141-1148.

[6] Debreczeni, J. É., Bunkóczi, G., Ma, Q., Blaser, H. \& Sheldrick, G. M. (2003). In-house measurement of the sulfur anomalous signal and its use for phasing. Acta Cryst. D59, 688-696.

[7] Debreczeni, J. É., Bunkóczi, G., Girmann, B. \& Sheldrick, G. M. (2003). In-house phase determination of the lima bean trypsin inhibitor: a low-resolution sulfur-SAD case. Acta Cryst. D59, 393-395.

[8] Neculai, D., Neculai, A.-M., Roesky, H. W., Magull, J. \& Bunkóczi, G. (2002). Synthesis and structure of a new fluorinated beta-ketoiminato ligand and its lithium derivative. J. Fluorine Chem. 118, 131-134.

[9] Wölfling, J., Frank, É., Mernyák, E., Bunkóczi, G., Cuesta Seijo, J. A. \& Schneider,

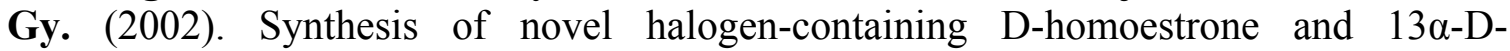
homoestrone derivatives by Lewis acid-induced intramolecular Prins reaction. Tetrahedron 58, 6851-6861.

[10] Lehmann, C., Bunkóczi, G., Vértesy, L. \& Sheldrick, G. M. (2002). Structures of glycopeptide antibiotics with peptides that model bacterial cell-wall precursors. J. Mol. Biol. 318, 723-732. 


\section{POSTERPRÄSENTATIONEN}

[1] Bunkóczi, G., Debreczeni, J. É., Sevvana, M. \& Sheldrick, G. M. (2003). Search for a test crystal. Strategies in Macromolecular Structure Determination at 3rd Generation Synchrotrons, June 17-25, Grenoble, France.

[2] Bunkóczi, G., Debreczeni, J. É., Sevvana, M., Vučković, M. \& Sheldrick, G. M. (2003). In-house phasing with iodide soaks. Workshop on Phasing with Soft X-rays, February 25-27, Brixen, Italy.

[3] Bunkóczi, G., Schiell, M., Vértesy, L. \& Sheldrick, G. M. (2002). Crystal Structures of cephaibols. Peptaibols: Biosynthesis, Structural Diversity, Bioactivity and Mode of Action, October 9-11, Jena, Germany.

[4] Bunkóczi, G., Debreczeni, J. É., Kärcher, J. \& Sheldrick, G. M. (2002). In-house phasing with iodide soaks. XIX. Congress and General Assembly of the International Union of Crystallography, August 6-15, Geneva, Switzerland.

[5] Bunkóczi, G., Lehmann, C., Vértesy, L. \& Sheldrick, G. M. (2001). High affinity binding to glycopeptide antibiotics. Sixth International School on the Crystallography of Biological Macromolecules, May 13-17, Como, Italy. 


\section{LEBENSLAUF}

\section{PERSÖNLICHE DATEN}

Name

Geburtsdatum

Geburtsort

Staatsangehörigkeit

Familienstand

SCHULBILDUNG

1991-1995
Gábor Bunkóczi

01. Oktober 1976

Debrecen, Ungarn

ungarisch

verheiratet

\section{HochSCHULSTUdIUM}

1995-2000

1999-2000

\section{Promotion}

2000-2004

Göttingen, den 16.03.2004

Gábor Bunkóczi
Eötvös Lorand Universität Budapest

Diplomarbeit am Lehrstuhl für Theoretische Chemie im Arbeitskreis von Prof. G. Náray-Szabó:

„Röntgenkristallographische Untersuchung von KalmodulinBisindolantagonist Komplexen"

Dissertation am Lehrstuhl für Strukturchemie der GeorgAugust-Universität Göttingen im Arbeitskreis von Prof. G.M. Sheldrick:

„Structure determination of peptides with antibacterial action” 